UNIVERSIDADE DE SÃO PAULO

FACULDADE DE FILOSOFIA, LETRAS E CIÊNCIAS HUMANAS

DEPARTAMENTO DE FILOSOFIA

LUCIANO VICENTE

Definições parciais de verdade e sistemas de acumulação na aritmética formal 
LUCIANO VICENTE

\section{Definições parciais de verdade e sistemas de acumulação na aritmética formal}

\section{Versão corrigida}

Tese apresentada ao Programa de Pós-graduação em Filosofia do Departamento de Filosofia da Faculdade de Filosofia, Letras e Ciências Humanas da Universidade de São Paulo para obtenção do título de Doutor em Filosofia.

Orientadora: Profa. Dra. Andréa Maria Altino de Campos Loparic

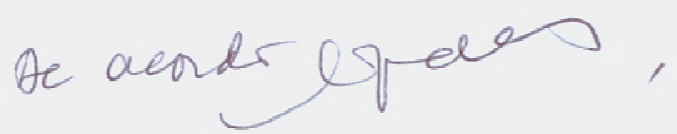

São Paulo 


\section{LUCIANO VICENTE}

Definições parciais de verdade e sistemas de acumulação na aritmética formal

Tese apresentada ao Programa de Pós-graduação em Filosofia do Departamento de Filosofia da Faculdade de Filosofia, Letras e Ciências Humanas da Universidade de São Paulo para obtenção do título de Doutor em Filosofia.

Orientadora: Profa. Dra. Andréa Maria Altino de Campos Loparic

São Paulo 



\section{Agradecimentos}

À professora Andréa pela confiança;

Aos professores Rodrigo A. Freire, Antônio M. N. Coelho e José Alexandre D. Guerzoni pelas críticas e sugestões;

Aos meus colegas e mestres pela inspiração;

Ao pessoal da secretaria pela solicitude;

Aos meus amigos e familiares pelo reconforto;

À Fapesp pelo financiamento;

À filosofia pela exasperação. 
"O la vile chose, dict-il, et abjecte, que l'homme, s'il ne s'eleve au dessus de l'humanité! Voylà un bon mot et un utile desir, mais pareillement absurde. Car de faire la poignée plus grand que le poing, la brassée plus grande que le bras, et d'esperer enjamber plus que de l'estanduë de nos jambes, cela est impossible et monstrueux." 


\section{Resumo}

VICENTE, Luciano. Definições parciais de verdade e sistemas de acumulação na aritmética formal. 2013. 148 f.. Tese (Doutorado)-Departamento de Filosofia da Faculdade de Filosofia, Letras e Ciências Humanas da Universidade de São Paulo, São Paulo, 2013.

Segundo o teorema da indefinibilidade de Tarski-Gödel, não existe fórmula da linguagem da aritmética que defina o conjunto dos números de Gödel das sentenças verdadeiras da aritmética. No entanto, para cada número natural $n$, podemos definir o conjunto dos números de Gödel das sentenças verdadeiras da aritmética de grau menor que $n$. Essas definições produzem uma hierarquia $\mathrm{V}_{0}(x), \mathrm{V}_{1}(x), \ldots, \mathrm{V}_{n}(x), \ldots$ tal que, para todo $x$, se $\mathrm{V}_{n}(x)$, então $\mathrm{V}_{n+1}(x)$. Nesse estudo, ensairemos algumas aplicações desses predicados, chamados definições parciais de verdade, e outros predicados relacionados a eles na construção de sistemas formais para as verdades da aritmética. A ideia subjacente aos nossos sistemas é muito simples, devemos acumular de alguma maneira as definições parciais de verdade. Grosso modo, mostrar como fazê-lo é o objetivo desse estudo.

Palavras-chave: Definições parciais de verdade, sistemas de acumulação. 


\begin{abstract}
VICENTE, Luciano. Partial truth definitions and accumulation systems in formal arithmetic. 2013. 148 f.. Thesis (Doctoral) -Departamento de Filosofia da Faculdade de Filosofia, Letras e Ciências Humanas da Universidade de São Paulo, São Paulo, 2013.

According to Tarski-Gödel's undefinability theorem, there is no formula in the language of arithmetic which defines the set of Gödel numbers of arithmetical true sentences. Nevertheless, for each $n$, we can define the set of Gödel numbers of all arithmetical true sentences of degree $n$ or less. These definitions yield a hierarchy of predicates $\mathrm{V}_{0}(x), \mathrm{V}_{1}(x), \ldots, \mathrm{V}_{n}(x), \ldots$ such that, for all $x$, if $\mathrm{V}_{n}(x)$, then $\mathrm{V}_{n+1}(x)$. In this study, we will ensay some aplications of these predicates, called partial truth definitions, and others related ones in building of formal systems for arithmetical truth. The underlying idea of our systems is very simple, we should accumulate in some way the partial truth definitions. Roughly speaking, showing how we can do that is the aim of this study.
\end{abstract}

Keywords: Partial truth definitions, accumulation systems. 


\section{Sumário}

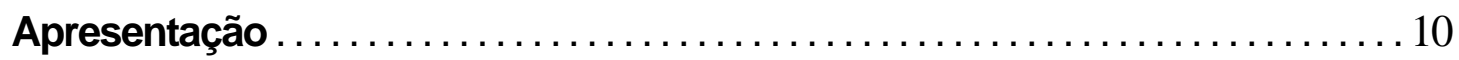

\section{Capítulo I:}

Da abordagem definicional à axiomática da verdade $\ldots \ldots \ldots \ldots \ldots \ldots \ldots \ldots$

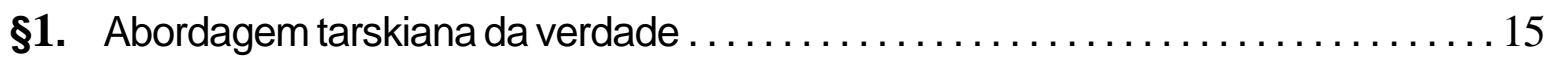

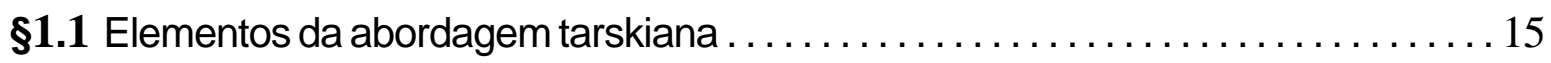

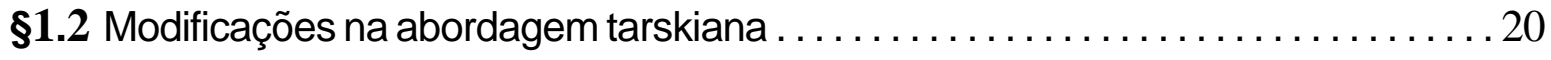

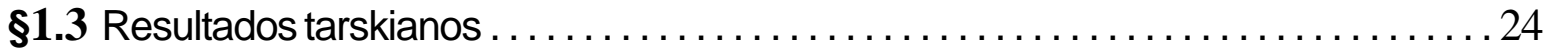

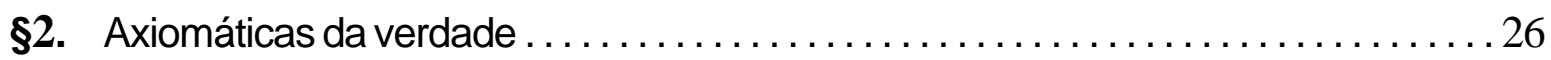

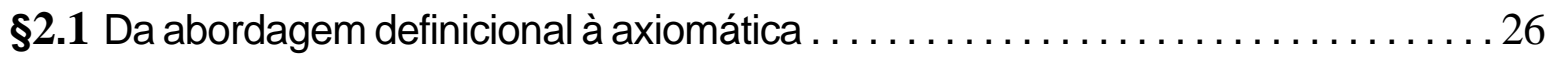

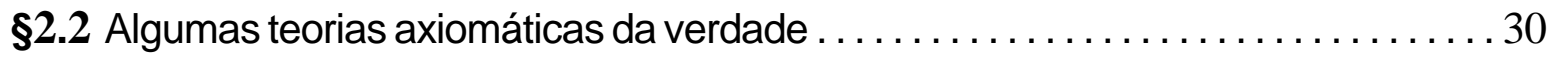

\$2.3 Demandas tarskianas ......................................... 34

\$2.4 Um argumento matemático e algumas alternativas teóricas ................ 36

\section{Capítulo II:}

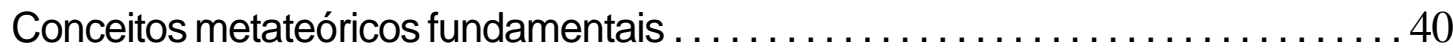

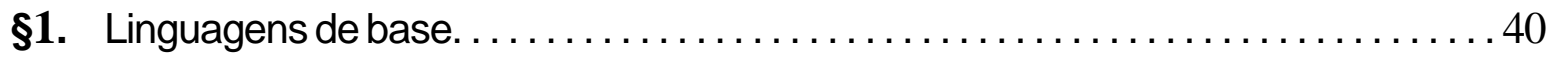

§2. Alguns conceitos semânticos ................................... 44

§3. Introdução à hierarquia da aritmética $\ldots \ldots \ldots \ldots \ldots \ldots \ldots \ldots \ldots \ldots \ldots \ldots$

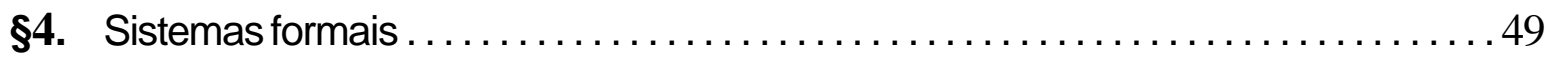

\section{Capítulo III:}

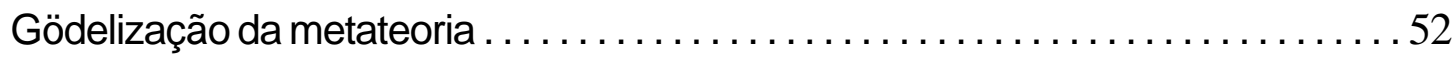

§1. Introdução à gödelização da sintaxe 1 : expressões $\ldots \ldots \ldots \ldots \ldots \ldots \ldots \ldots 2$

\$2. Coletânea de contrapartidas formais relevantes ..................... 53

§3. Codificação da teoria dos conjuntos e das sequências finitas .............. 56 
§4. Introdução à gödelização da sintaxe 2: termos $\ldots \ldots \ldots \ldots \ldots \ldots \ldots \ldots \ldots$

§5. Introdução à gödelização da semântica: fórmulas-legenda ................ 64

§6. Introdução à gödelização da sintaxe 3: sentenças ....................6 65

\$7. Resultados relativos às contrapartidas formais . ..................... 70

\section{Capítulo IV:}

Definições parciais de verdade e falsidade $\ldots \ldots \ldots \ldots \ldots \ldots \ldots \ldots \ldots \ldots \ldots \ldots \ldots \ldots$

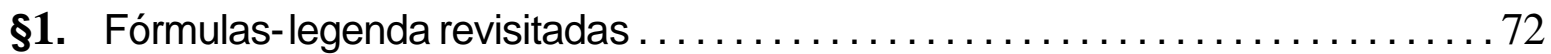

\$2. Definição de verdade para sentenças atômicas . ..................... 76

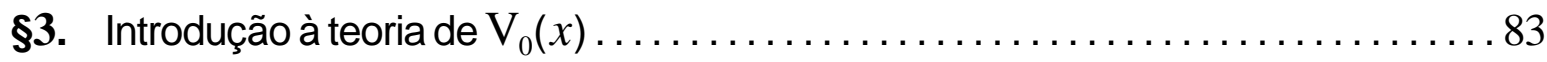

§4. Outros tipos de definições parciais de verdade ...................... 91

§5. Definição de verdade para sentenças de complexidade $n \ldots \ldots \ldots \ldots \ldots 92$

§6. Introdução à teoria das definições parciais de verdade .................96

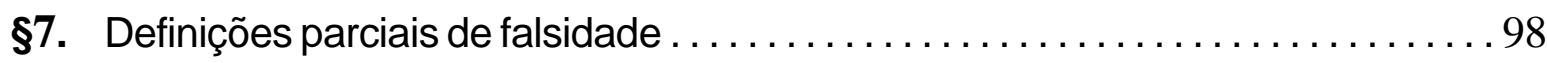

\section{Capítulo V:}

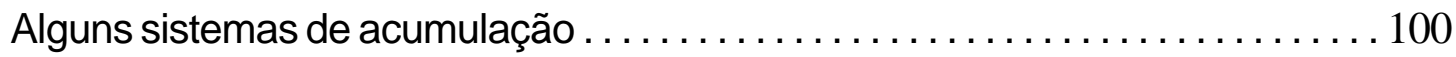

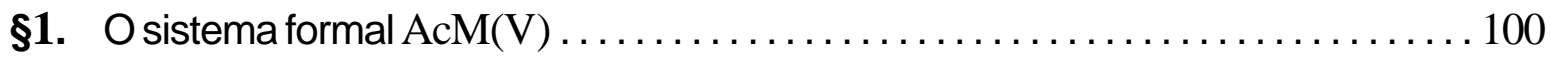

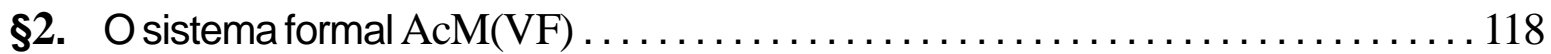

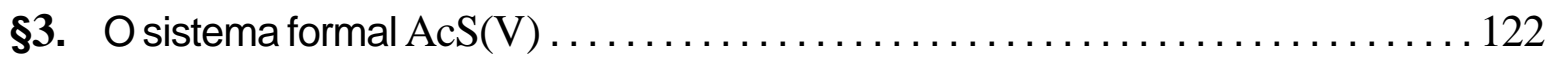

\section{Capítulo VI:}

Sistemas baseados em definições parcias alternativas . ............... 124

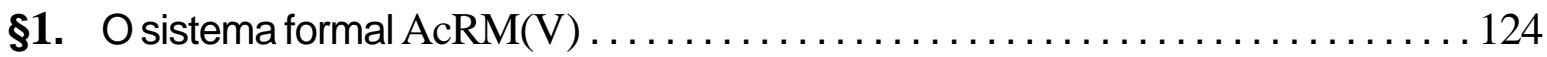

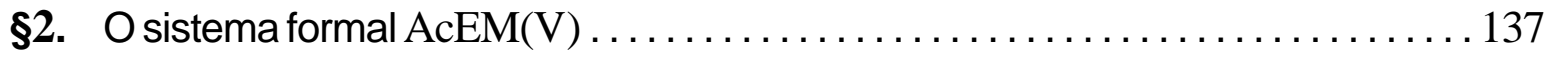

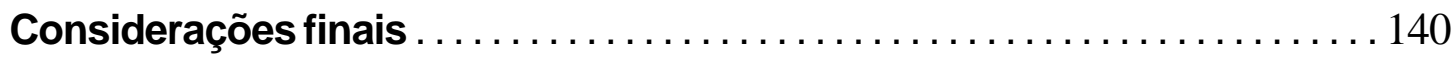

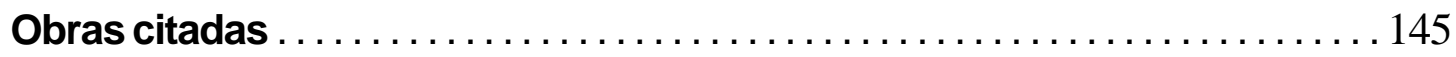




\section{Apresentação}

Segundo Boolos e Jeffrey em Computability and Logic [1980, p. 207]: o teorema da indefinibilidade de Tarski é "[...] um resultado negativo [...] posto, por assim dizer, entre dois resultados positivos: de um lado, cada uma de certas 'aproximações' $\mathrm{V}_{n}$ de $\mathrm{V}$ [onde $\mathrm{V}$ é o conjunto de números de Gödel das sentenças verdadeiras da aritmética] é definível na aritmética [de primeira-ordem], e, de outro, $\mathrm{V}$ é ele mesmo 'definível na aritmética de segunda-ordem'”.

Ensaiaremos, nesse estudo, algumas possíveis aplicações dessas 'aproximações' $\mathrm{V}_{n}$-ou, como as chamaremos (seguindo Hájek e Pudlák [1998, p. 51]), definições parciais de verdade (mais sucintamente, DPVs) - na construção de sistemas formais para o "predicado de verdade" da aritmética. (Não devemos confundir nossas DPVs com os bicondicionais de Tarski, ou seja, com sentenças da forma $\varphi \leftrightarrow \mathrm{V}(\lceil\varphi\rceil)$, cuja designação original de Tarski é a mesma [1935, p. 268; 1956, p. 155].)

Nossos sistemas-ignoraremos nessa apresentação os sistemas referentes ao predicado de falsidade da aritmética-serão sistemas formais nos quais introduziremos, ao lado das constantes aritméticas costumeiras, um nova constante $V$, cuja interpretação pretendida (intended interpretation) será o conjunto de números de Gödel das sentenças verdadeiras no modelo padrão da aritmética de primeira-ordem.

Para estudá-los, precisaremos, pelo menos, vislumbrar uma certa aritmética, de sutilezas e detalhes, que é implicada pelas DPVs e pelas noções formais correlatas de sequência de valoração e denotação. Feliz e incidentalmente, a utilidade dessa aritmética "em filigrana", apesar das dificuldades que Ihe são inerentes, não se resume às aplicações que dela faremos; ela remete a questões meta-aritméticas mais "tradicionais": é uma ferramenta essencial à formalização de fragmentos da teoria dos conjuntos e da análise combinatória na aritmética de primeira-ordem; além disso, é parte essencial tanto do 
argumento que estabelece que P.A. não é finitamente axiomatizável quanto da teoria dos modelos desviantes (non-standard) de P.A..

Boa parte da literatura lógico-filosófica acerca da verdade visa, parafraseando Hartry Field, salvá-la dos paradoxos ou, pelo menos, impedir-lhes as consequências nefastas (como em In Contradiction de Priest [2006a, passim]). Deixemos claro que esse estudo não é, em sua gênese, fruto de reflexões sobre a natureza desses famosos paradoxos, e isso, de certa maneira, determinará sua feitura e realização; ele é, contrastantemente, resultado de considerações metateóricas. Mais especificamente, a pré-história desse estudo está ligada à análise das limitações intrínsecas aos sistemas formais iniciadas por Gödel e ao encontro de certos procedimentos de extensão de formalismos anunciados na obra desse autor e propostos mais detida e rigorosamente por Turing em 'Systems of Logic Based on Ordinals' [1939] e Feferman em 'Transfinite recursive progressions of axiomatic theories' [1962].

Desse modo, as DPVs aparecem em princípio como instrumentos convenientes para a realização de certos tipos de extensões da aritmética de primeira-ordem e, de fato, poderíamos pensar (e iremos fazê-lo) em várias maneiras de "acumularmos" tais aproximações.

É nesse contexto das teorias de acumulação dos predicados parciais de verdade que as aplicações das DPVs à definição de sistemas formais serão examinadas.

Em suma, não estamos em princípio nos opondo a nenhuma das diversas teorias axiomáticas da verdade estabelecidas em outros contextos, estamos tão-somente propondo alguns sistemas formais que surgem naturalmente da consideração das DPVs (de modo análogo, os teoremas da incompletude sugerem extensões por meio dos chamados "princípios de reflexão"). Não está em causa se os sistemas formais que definiremos são ou não, de alguma maneira, superiores aos demais; algo nesse sentido demandaria uma argumentação independente. 
De fato, a equivalência entre nossos sistemas e outros propostos em diferentes contextos seria um resultado bem-vindo, na medida em que apontaria para certa invariância no conceito de verdade (de maneira análoga, a equivalência das diversas formalizações do conceito de computabilidade corrobora a Tese de Church-Turing).

Obviamente, poderíamos polemizar e, contra o mote "truth is disquotation", ratificar: "Verdade é acumulação (de verdades parciais)"; mas, em alguns contextos, os motes parecem se sustentar mutuamente. Em todo caso, nossa pergunta não será se a verdade são acumulações (embora nossa pesquisa progressivamente nos conduza nessa direção), mas sim: como acumulá-las? Falando claramente, esse estudo pretende apresentar os rudimentos de uma resposta a essa questão.

No Capítulo I, Da abordagem definicional à axiomática da verdade, contextualizaremos, contrapondo-as rapidamente à abordagem definicional de Tarski, as axiomáticas do "predicado de verdade" em geral e os sistemas formais propostos nesse estudo em particular.

No Capítulo II, Conceitos metateóricos fundamentais, introduziremos as noções de linguagem de base, de denotação e verdade no modelo padrão da aritmética, bem como o sistema formal P.A., além de outros conceitos a eles relacionados tais como "expressão" e "representação". Nada aqui foge muito das exposições costumeiras (apenas, por razões de ordem técnica, incorporamos a exponenciação como primitiva). O propósito desse capítulo será basicamente definitório.

No Capítulo III, Gödelização da metateoria, empreenderemos a aritmetização de conceitos metateóricos fundamentais para nossa discussão posterior, dando especial atenção às "fórmulas-legenda", ou seja, às contrapartidas formais do conceito de denotação, que serão a base de nossas definições parciais de verdade e falsidade. 
No Capítulo IV, Definições parciais de verdade e de falsidade, apresentaremos nossas DPVs e DPFs (definições parciais de falsidade); e estabeleceremos alguns resultados da teoria que lhes é subjacente.

No Capítulo V, Alguns sistemas de acumulação, introduziremos sistemas formais, nos quais as DPVs e DPFs têm papel essencial; estabeleceremos vários de seus resultados mais notáveis e os compararemos rapidamente com outros sistemas similares já presentes na literatura.

No Capítulo VI, Sistemas baseados em definições parciais alternativas, apresentaremos duas novas formas de definições parciais de verdade, a saber: as definições enraizadas ou, sucintamente, RVs e as definições estruturais ou EVs; além de introduzirmos os respectivos sistemas minimais de acumulação correlatos dessas novas definições.

Nas Considerações finais, indicaremos, tanto do ponto de vista filosóficoespeculativo quanto do ponto de vista lógico-sistemático, algumas das inúmeras direções de pesquisa que nossos sistemas de acumulação parecem sugerir.

As DPVs tal como serão apresentadas no capítulo IV desse estudo são baseadas em definições parciais de verdade que aparecem tanto em Boolos \& Jeffrey [1980, pp. 207 e segs.] quanto em Hájek \& Pudlák [1998, pp. 51-61], enquanto nossas DPFs (definições parciais de falsidade) são modificações óbvias das DPVs.

Por outro lado, nossas definições parciais enraizadas ou RVs [cf. p. 127] e nossas definições parciais estruturais ou EVs [cf. p. 137] que serão introduzidas no capítulo VI, se não inéditas na literatura (e tudo indica que elas sejam), são, pelo menos, resultado de um esforço independente de nossa parte. O mesmo deve ser dito de todos os sistemas de acumulação apresentados nesse estudo (e, portanto, dos resultados que lhes são relativos). 
Tarski escreveu no prefácio de Introduction to Logic and the Methodology of Deductive Sciences [1994, p. ix]: "[...] mathematics is expanding its realm in all possible directions, it is growing in height, in width, and in depth [...]".

Poderíamos e deveríamos expandir também esse estudo em todas direções sugeridas ali por Tarski: in height, alcançando novos resultados; in width, estabelecendo comparações mais abrangentes; $\mathrm{e}$, in depth, fundamentando mais adequadamente nossa discussão. E, talvez, para além da materialidade do que se segue (e, até mesmo, de sua constituição como etapa de formação de um, esperamos, pesquisador e professor de lógica), possamos julgar esse estudo também pela possível fecundidade.

Observação. Boa parte de nossa pesquisa foi realizada no âmbito abrangente de vários temas propostos pelos métodos gödelianos de aritmetização da sintaxe e de auto-referência indireta, e, como seria de se esperar, muito dessa pesquisa não aparecerá explicitamente no que se segue. Entretanto, algumas idiossincrasias derivadas dessa "abrangência" irão estar presentes no modo de exposição de nossos resultados: por exemplo, o uso de um alfabeto finito para gerar as expressões de nossas linguagens de base (cf. p. 40) e da concatenação na base 10 como função básica do processo de aritmetização (cf. p. 52) não são particularmente utéis no contexto desse estudo, mas se justificariam em contextos mais amplos. 


\section{Da abordagem definicional à axiomática da verdade}

\section{$\S 1$. Abordagem tarskiana da verdade}

\section{§1.1. Elementos da abordagem tarskiana}

\section{Em 'The Concept of Truth in Formalized Languages'(1), Alfred Tarski se propõe a} enfrentar uma antiga questão filosófica: o problema da verdade. Em termos à primeira vista menos pretensiosos do que sugeriria o peso da tradição, o autor se coloca a tarefa de construir, apoiando-se no conceito de metalinguagem associada a uma linguagem formalizada dada ${ }^{(2)}$, uma definição materialmente adequada e formalmente correta da função sentencial “... é uma sentença verdadeira”(3).

1. O artigo de Tarski aparece na versão polonesa em Ruch Filozoficzny, vol. XII (1930-1); na versão alemã, 'Der Wahrheitsbegriff in den formalisierten Sprachen', em Studia philosophica, vol. I, 1935, pp. 261-405; e, em tradução inglesa de J. H. Woodger, 'The Concept of Truth in Formalized Languages', em Logic, Semantics, Metamathematics, 1956, pp. 152-278. Nossa principal fonte nesse estudo preliminar será a tradução de Woodger, entretanto, a versão alemã será ocasionalmente empregada.

2. Segundo Tarski [1956, pp.154-165], a incompatibilidade entre as leis da lógica clássica e a universalidade característica das linguagens naturais coloca "dificuldades insuperáveis" à construção de uma definição de verdade adequada às linguagens naturais. Deveríamos, portanto, nos restringir, nas palavras do autor, "inteiramente às linguagens formalizadas" [ibidem, p. 165].*

* Existem, entretanto e como seria de se esperar, muitos filósofos que recusam as conclusões de Tarski; Scott Soames [1999, p. 56], tipicamente e por exemplo, diz que, não importa quão plausíveis sejam as assunções tarskianas, "[...] elas são conjuntamente incoerentes e, portanto, inaceitáveis. De modo que a tarefa de encontrar princípios mais acurados e aceitáveis [que aqueles pressupostos no argumento de Tarski] permanece".

3. Trata-se, para Tarski [1956, p. 153], da concepção clássica da verdade em oposição, por exemplo, às concepções utilitaristas. De fato, a análise de Tarski parte da definição "clássica" (ou correspondencial) de verdade, a primeira formulação do autor é a seguinte: "(1) a true sentence is one which says that the state of affairs is so and so, and the state os affairs is so and so." [ibidem, p. 155]; o que Ihe sugere uma comparação entre (1) e a formulação propriamente aristotélica da Metafísica [IV, 7, 1011b, 1969, p.107]: "Falso é dizer que o que é não é, ou que o que não é, é; verdadeiro é dizer que o que é, é e o que não é não é [...]". Entretanto, tal análise rapidamente adquire contornos mais abstratos; Tarski propõe, poucas linhas abaixo da primeira formulação, uma segunda: "(2) $x$ is a true sentence if and only if $p$ ", onde $x$ deve ser um nome da sentença $p$. Presumivelmente, uma formulação de caráter tão abstrato quanto essa poderia, além de acomodar variantes da concepção correspondencial, ser compatível, à revelia de Tarski, com algumas concepções de verdade nãocorrespondenciais-mas não, possivelmente, com qualquer uma delas, e.g., com concepções coerenciais (de fato, a discussão desse ponto se torna frequentemente bastante sutil e difícil, acabando por envolver tanto argumentos de natureza epistemológica quanto, mais propriamente, ontológicos [cf., por exemplo, Kirkham, 2001, pp. 182-183]). 
Mais especifica e analiticamente, Tarski parte de:

a) uma ciência dedutiva (deduktive Wissenschaft, deductive science) $\mathrm{A}$;

b) definida na linguagem formalizada (formalisiert Sprache, formalized language) L;

c) cuja atribuição I de sentido-significado (Sinn-Bedeutung, sense-meaning) às constantes não-lógicas de Lestaria pressuposta e seria compatível com $\mathrm{A}^{(4)}$.

Para, então, construir:

a) uma linguagem formalizada $L^{\prime}, L^{\prime}$ é a metalinguagem tarskiana de $L$;

b) uma ciência dedutiva $A^{\prime}, A^{\prime}$ é a metaciência tarskiana de $A$;

c) uma fórmula $\mathrm{V}(x)$ de $\mathrm{L}^{\prime}, \mathrm{V}(x)$ é a definição tarskiana de verdade em $\mathrm{L}^{\prime}$ (conformel);

d) Uma função $n$ das sentenças de $L$ em certos termos de $L^{\prime}, n(\alpha)$ é o nome descritivo-estrutural $^{(5)}$ de Tarski da sentença $\alpha$, de L, em L';

e) Uma função $t$ das fórmulas de $L$ em certas fórmulas de $L^{\prime} ; t(\alpha)$ é a tradução tarskiana da fórmula $\alpha$, de $L$, em L'.

No caso particular da construção apresentada no artigo de Tarski, $L^{\prime}$ incorpora termos correspondentes às expressões de $L$ (os nomes descritivos-estruturais), bem como operações que possibilitam um tratamento do conteúdo estrutural desses termos; tais operações, por sua vez, são descritas usando operações aritméticas; de modo que $A^{\prime}$ deve, então, incorporar axiomas regulando as operações envolvidas, tanto estruturais quanto aritméticas ${ }^{(6)}$.

4. A atribuição I e a ciência dedutiva $A$ devem ser, por assim dizer, adequadas uma à outra, abusando um pouco da terminologia atual, diríamos que A deve axiomatizar, pelo menos alguns dos "aspectos", de I e que I deve ser um modelo de $\mathrm{A}$.

5. Segundo Tarski, nomes descritivos-estruturais "[...] describe the words which compose the expression denoted by the name [...]" [1956, pp. 156-157]. Entretanto, no caso mais específico das linguagens formalizadas, os nomes descritivos-estruturais não "descrevem" própria e imediatamente "the words which compose ..."; a descrição é, por assim dizer, mediada pelas contrapartidas formais de predicados e relações tais como " $x$ é 0 símbolo de negação", " $x$ é o símbolo de quantificação universal", " $x$ é a concatenação entre $y$ e $z$ ", " $x$ é uma disjunção cujos disjuntos são, respectivamente, $y$ e $z$ ", etc. [cf. ibidem, pp.172-174]. 
Definição A. Diremos que $\left\langle L, A, I \mid L^{\prime}, A^{\prime}, n, V(x)\right\rangle$ é uma estrutura tarskiana, cujas estruturas de partida e de chegada são $\langle L, A, I\rangle$ e $\left\langle L^{\prime}, A^{\prime}, n \dagger V(x)\right\rangle$, respectivamente. ${ }^{(7)(8)}$

Temos, assim, que:

Teorema da Definibilidade de Tarski. Para qualquer sentença $\alpha$ de $L$, se $\tau$ é $\mathrm{n}(\alpha)$ e $\varphi$ é $\mathrm{t}(\alpha)$, então $\vdash \mathrm{A}^{\prime} \varphi \leftrightarrow \mathrm{V}(\tau)$

O ponto mais importante da descrição esquemática acima é que devemos compreender que todos os elementos de uma estrutura tarskiana estão intrinsecamente relacionados. O sentido da definição tarskiana de verdade, por exemplo, é determinado apenas por intermédio dos nomes descritivos-estruturais e da tradução correspondentes (o enunciado do Teorema da Definibilidade é claro em relação a esse ponto). De um lado, portanto, temos que, dada uma estrutura de partida $\langle L, A, I\rangle$, podemos construir $\left\langle L^{\prime}, A^{\prime}, n t V(x)\right\rangle$; de outro, temos que o sentido dos elementos da estrutura de chegada é determinado apenas na medida em que eles são considerados conjuntamente ${ }^{(9)}$.

6. No caso estrutural, isso é dado explicitamente pelos Axiomas 1-5 [Tarski, 1956, pp. 173-174]; no caso aritmético, Tarski assume implicitamente os axiomas usuais da aritmética [ibidem, p.171].

7. Podemos ver $\left\langle L, A, I \mid L^{\prime}, A^{\prime}, n, t, V(x)\right\rangle$ como uma abreviação do par ordenado formado, respectivamente, pelas estruturas de partida e chegada, ou seja, como $\left\langle\langle L, A, I\rangle,\left\langle L^{\prime}, A^{\prime}, n, t, V(x)\right\rangle\right\rangle$.

8. A distinção entre estruturas de partida e chegada torna bastante clara a dependência da definição de verdade, $\mathrm{V}(x)$, em relação à atribuição I . Grosso modo, a verdade é parasitária do significado; Tarski [1956, p. 167] diz textualmente: "We shall always ascribe quite concrete and, for us, intelligible meanings to the signs wich occur in the languages we shall consider". De fato, a tradução tarskiana depende da introdução do domínio associado a $L$ por I como predicado metalinguístico: Seja $\left\langle L_{1}, A_{1} I_{1}\right\rangle$ uma estrutura de partida na qual $L_{1}=\left\{\langle\}, \operatorname{Dom}\left(I_{1}\right)=0\right.$ conjunto de números reais, $\mathrm{I}_{1}(<)=$ a ordem linear estrita dos reais; $\mathrm{t}(\forall x(x<x))$ seria, então, uma contrapartida formal em $L_{1}^{\prime}$ de "para todo número real $a, a$ é menor que $a$ "; notemos a relativização de " $x$ é menor que $y$ ". No caso de $\mathrm{V}(x)$ o mesmo acontece, $\mathrm{V}(\mathrm{n}(\forall x \exists y(x<y)))$ será derivável em $\mathrm{A}_{1}^{\prime}$ se e somente se uma contrapartida formal de "para qualquer sequência infinita $s_{1}$ de números reais, existe uma sequência $s_{2}$ que é diferente $s_{1}$, possivelmente, apenas com respeito ao segundo membro e tal que o primeiro membro de $s_{2}$ é menor que o segundo" é derivável em $\mathrm{A}_{\mathrm{i}}^{\prime}$ Em suma, a definição tarskiana da verdade, $\mathrm{V}(x)$, não deveria ser tomada como explicação do conceito de significado (pelo menos, não sem modificações); pois, nela, de alguma maneira (no nosso exemplo, por meio de $\mathrm{I}_{1}$ ), o significado das expressões é tomado como um dado. É o que Kirkham [2001, pp. 178-181], argumentando em outro contexto e de maneira alternativa, sustenta; Davidson em 'Truth and Meaning' [2006, pp. 155-170], por sua vez, rearranja completamente os parâmetros do problema. 
Dado o background atualmente compartilhado pelos lógicos e comparada às versões mais recentes, a exposição original de Tarski apresenta algumas dificuldades. De fato, não é fácil precisar aquilo que Tarski quer dizer com deduktive Wissenschaft, formalisiert Sprache ou com o par Sinn-Bedeutung (usados indiscriminadamente) e mesmo nossa atribuição de certas "posições" na estrutura tarskiana-apelando às variáveis L, A e I -parece ser passível de crítica.

Questões como a da natureza precisa da deduktive Wissenschafte da formalisiert Sprache tarskianas são, obviamente, de interesse tanto histórico quanto conceitual e, de modo algum, seria lícito simplesmente varrê-las para baixo do tapete (o que não afasta a possibilidade de mostrarmos que tal precisão é, de fato, impossível). Iremos, portanto, conscientes das dificuldades intrínsecas a essas questões, apresentar, com vistas à discussão, um esquema alternativo ao proposto anteriormente mais ao gosto atual, esperando, é claro, que o possível caráter polêmico dessa "atualização" não prejudique nossos propósitos argumentativos mais imediatos.

Para efeito de discussão, nossa estrutura de partida será formada por:

a) Uma linguagem formal $L$;

b) Um sistema formal A definido em L;

c) Um modelo I de $A^{(10)}$.

No nosso caso particular, aos conceitos de linguagem e sistema formal devem, no espírito hilbertiano, corresponder conceitos decidíveis de fórmula e prova ${ }^{(11)}$.

9. No caso de Tarski, o bom funcionamento conjunto da estrutura é, em última instância, apoiado por considerações de ordem intuitiva: $L^{\prime}$ é realmente (o que poderíamos pensar como) uma metalinguagem para $L$ (uma vez que, entre outras coisas, a capacidade expressiva de $L^{\prime}$ é superior a de $L$ e que $L^{\prime}$ incorpora termos descritivos-estruturais como nomes de expressões de $L$ ); $A^{\prime}$ é realmente (o que poderíamos pensar como) uma metaciência associada a $A$ e $L$; té realmente uma tradução das fórmulas de $L$ em $L^{\prime}$, etc..

10. Presumivelmente, algumas restrições deveriam ser impostas a essas interpretações. Por exemplo, a classe de todos os conjuntos poderia ser tomada como domínio de tais interpretações? e quanto ao "superagregado" de todas as classes (próprias ou não)? Uma discussão dessas questões pode ser encontrada, por exemplo, em Saving Truth from Paradox de Hartry Field [2008, pp. 33-36].

11. Cp. o conceito de "axiomatic formal theories" de Mendelson [1997, p. 34]. 
Uma alternativa seria aceitar uma concepção, sob certo ponto de vista, mais abrangente de "sistema formal" como a de Shoenfield ${ }^{(12)}$; contudo e apesar das reservas, sustentaremos, por assim dizer, oficialmente o esquema acima.

De fato, algumas referências de Tarski a certas "descrições estruturais" (structural descriptions [1956, p. 166], strukturelle Beschreibung ${ }^{(13)}$ [1935, p. 280]) de símbolos e axiomas apoiam ligeiramente nossa "atualização".

Poderíamos, segundo esse ponto de vista, apresentar os símbolos de uma linguagem formalizada ou por meio de uma lista finita ou por meio de uma lista infinita "descrita estruturalmente" [cf. itens $(\alpha)$ e $(\beta)$, idem, 1956, p. 166]. Nesse sentido, os símbolos de predicados da linguagem seriam, e. g., da forma $R_{j}^{i}$, onde $i$ e $j$ são inteiros positivos e representam, respectivamente, a aridade e a posição do símbolo na lista.

No caso da ciência dedutiva relativa à estrutura de partida, ou os axiomas seriam introduzidos por uma lista finita ou por meio de, diríamos hoje, esquemas de axiomas [cf. item $(\gamma)$, ibidem, p. 166].

Partindo da estrutura acima, construiríamos, então:

a) Uma linguagem formal $L^{\prime}, L^{\prime}$ é a metalinguagem tarskiana de $L$;

b) Um sistema formal $A^{\prime}$ definido em $L^{\prime}, A^{\prime}$ é a metateoria tarskiana de $A$;

c) Uma fórmula $\mathrm{V}(x)$ de $\mathrm{L}^{\prime}, \mathrm{V}(x)$ é a definição tarskiana de verdade de I em $\mathrm{L}^{\prime}$;

d) Uma função $n$ das sentenças de $L$ em certos termos de $L^{\prime}, n(\alpha)$ é o nome descritivo-estrutural de Tarski da sentença $\alpha$, de L, em L';

e) Uma função $t$ das fórmulas de $L$ em certas fórmulas de $L^{\prime} ; t(\alpha)$ é a tradução tarskiana da fórmula $\alpha$, de $L$, em $L^{\prime}$.

12. Para Shoenfield [cf. 1967, pp. 3-6], o conjunto de teoremas de um sistema formal é, grosso modo, o fecho transitivo de um conjunto qualquer de fórmulas (axiomas) segundo relações dadas (regras de inferência), tomadas elas próprias conjuntamente.

13. No caso, "strukturelle Beschreibung" foi adaptado, uma das referências é mais precisamente: "[...] gibt man an oder beschreibt strukturell eine Kategorie von Aussagen, welche man Axiome oder Grundsätze nennt." [1935, p. 280]. 


\section{§1.2. Modificações na abordagem tarskiana}

Seja qual for o valor da abordagem definicional de Tarski tomada como "elucidação" ou "explicação" do conceito de verdade, uma de suas vantagens é a flexibilidade; com efeito, as estruturas tarskianas permitem (ou, talvez melhor, suportam) uma série de modificações tanto fecundas do ponto de vista teórico quanto úteis do ponto de vista técnico. Do ponto de vista técnico, podemos, em boa parte dos casos (leia-se: em boa parte das possíveis estruturas de partida), substituir-e iremos fazê-lo no caso de $\left\langle L_{\text {P.A. }}\right.$ P.A., $\left.I_{\mathbb{N}}\right\rangle$ - uma metateoria estritamente tarskiana pela teoria dos conjuntos (ficando, assim, a metalinguagem dispensada da quantificação sobre variáveis de ordem superior $\left.{ }^{(14)}\right)$. Do ponto de vista mais propriamente teórico, podemos, por exemplo, estabelecer algumas correlações interessantes entre a força expressiva de uma linguagem e a possibilidade de nela definirmos "predicados de verdade".

Entretanto, parece óbvio que essa flexibilidade não pode ser absoluta, o que coloca uma questão prévia: Quais seriam as modificações permitidas, uma vez que não queremos trair o espírito da abordagem de Tarski?

Felizmente, para nossos objetivos mais imediatos, uma resposta incompleta será suficiente.

Começaremos ignorando: a) a distinção entre as estruturas de partida e de chegada, b) assim como qualquer vínculo construtivo explícito entre os elementos da estrutura.

Seja $a=\left\langle L_{1}, A_{1} I_{1}, L_{2}, A_{2}, n_{1}, t_{1}, V_{1}(x)\right\rangle$ tal estrutura. A questão se torna, então: Quais condições deveriam ser satisfeitas por a para que os elementos de arepresentassem papéis similares aos seus correlatos mais estritamente tarskianos?

14. No nosso caso específico, deveremos substituir a metateoria pela teoria pura dos conjuntos, de modo que a tradução do domínio de nossa interpretação seja, na metalinguagem, um conjunto puro. Outra possibilidade é substituirmos os recursos de ordem superior por uma teoria dos conjuntos na qual os elementos do domínio da interpretação são introduzidos como átomos ou indivíduos (Urelemente). 
Ora, para a maioria dos casos a resposta é simples, as condições são tanto intuitivamente imediatas quanto relativamente fáceis de justificar:

a) $A_{1}$ deverá ser um sistema ${ }^{(15)(16)}$ definido em $L_{1}$ e I ${ }_{1}$ deverá ser um modelo de $A_{1}$;

b) $A_{2}$ deverá ser um sistema definido em $L_{2}$;

c) $t_{1}$ deverá ser uma interpretação de $A_{1}$ em $A_{2}{ }^{(17)}$;

d) $\mathrm{n}_{1}$ e $t_{1}$ deverão ser funções calculáveis e injetoras;

e) Um análogo do teorema da definibilidade deverá ser satisfeito por $\mathrm{a}^{(18)}$

Agora, com algumas dessas condições em mente, podemos, por assim dizer, fixar mais completamente os elementos de nossas "estruturas de partida".

Um exemplo extremo dessa "fixação" é, primeiramente, a) tomarmos, como estrutura de partida, $b=\left\langle L_{1}, A_{1}, I_{1}, L_{2}, A_{2}, X y\right\rangle$ (na qual a linguagem, a interpretação, 0 sistema, a metalinguagem, a metateoria, os nomes descritivos-estruturais e a tradução estão todos já determinados) b) para, então, construirmos uma definição de verdade $\mathrm{V}(x)$ para $\mathrm{b}$

É nesse espírito que podemos construir uma definição de verdade, cujos pormenores seguem de perto as indicações de Tarski, e que toma P.A. como teoria e ZF como metateoria.

15. Por um sistema, entenderemos um conjunto de sentenças fechado sob uma relação de consequência lógica supostamente dada. Não discutiremos, contudo, se e como uma mesma relação de consequência deve, por assim dizer, ser compartilhada pelos diferentes elementos da estrutura (a questão é sutil, mas não será relevante para nossa discussão).

16. A possibilidade de, no lugar de sistemas, utilizarmos teorias (no sentido de um conjunto qualquer de sentenças) será simplesmente ignorada.

17. Não devemos confundir uma interpretação do sistema $A_{1}$ no sistema $A_{2}$ com uma interpretação da linguagem L. Para interpretarmos $A_{1}$ em $A_{2}$, devemos, grosso modo, adicionar "definições possíveis" das constantes de $A_{1}$ como axiomas de $A_{2}$ e verificar que tais definições se comportam como deveriam, ou seja, que os teoremas de $A_{1}$ são deriváveis em $A_{2}$ módulo tal interpretação [cf. Undecidable Theories de Tarski, Mostowski e Robinson, 1971, pp.20-22].

18. Infelizmente, uma lista exaustiva e rigorosa das condições não é tão fácil de se conseguir. Um pequeno descuido e um pouco de criatividade permitiriam, por exemplo, distorcer o conteúdo intuitivo do teorema da definibilidade até torná-lo irreconhecível. 
Um pouco mais detalhadamente:

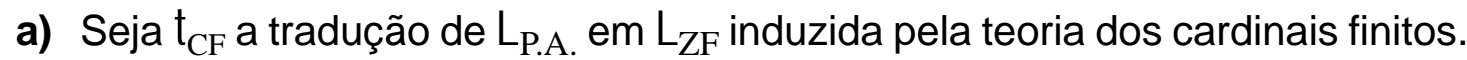

b) Seja * \lceil\rceil$^{*}$ a adaptação para $L_{Z F}$ de uma função metalinguística que aritmetize, à maneira de Gödel, as expressões de $L_{P . A}$.

A estrutura $\mathrm{S}=\left\langle\mathrm{L}_{\text {P.A. }}\right.$, P.A., $\left.\mathrm{I}_{\mathbb{N}}, \mathrm{L}_{\mathrm{ZF}}, \mathrm{ZF},{ }^{*}\lceil\rceil^{*}, \mathrm{t}_{\mathrm{CF}} \mid \mathrm{V}(x)\right\rangle$, onde $\mathrm{I}_{\mathbb{N}}$ é a interpretação usual (ou padrão) das constantes de $\mathrm{L}_{\mathrm{P} . \mathrm{A}}$, torna-se, então, comparável à estrutura tarskiana $\left\langle\mathrm{L}_{\text {P.A. }}\right.$, P.A., $\left.\mathrm{I}_{\mathbb{N}}\right| \mathrm{L}_{\text {P.A., }}^{\prime}$ P.A., $\left.\mathrm{n} \mathrm{t} \mathrm{V}(x)\right\rangle$, na medida em que os elementos correlatos de cada estrutura cumprem papéis semelhantes.

Em particular, assim como no caso da estrutura mais estritamente tarskiana, temos um teorema de definibilidade para S:

Definibilidade $\left\langle\right.$ P.A., $\left.\mathrm{I}_{\mathbb{N}}, \mathbf{Z F}\right\rangle$ de Tarski. $\vdash_{\mathrm{ZF}} \mathrm{t}_{\mathrm{CF}}(\alpha) \leftrightarrow \mathrm{V}\left({ }^{*}\lceil\alpha\rceil^{*}\right)$, para qualquer sentença $\alpha$ de $L_{P . A}$.

Além disso, a "fixação" dos elementos da estrutura de partida é particularmente útil para o estabelecimento de alguns resultados "negativos" ou "limitativos".

Sabemos, por exemplo, que os métodos de Gödel de aritmetização da sintaxe permitem que P.A. seja tomado como sua própria metateoria. Seguindo este caminho:

a) Seja $\Delta$ a função identidade (ou diagonal) das expressões de $L_{P . A . ;}$;

b) Seja \lceil\rceil a função gödeliana que aritmetiza as expressões de $L_{P . A}$.

Tomemos, agora, $\left\langle L_{\text {P.A. }}\right.$ P.A., $I_{\mathbb{N}}, L_{\text {P.A. }}$ P.A., \lceil\rceil$\left., \Delta\right\rangle$ como estrutura de partida (no caso, L.A. é tomada tanto como linguagem quanto como metalinguagem, P.A. é tomado tanto como sistema quanto como "meta-sistema").

Podemos, então, enunciar um famoso resultado limitativo:

Indefinibilidade P.A. de Gödel-Tarski. Se P.A. é consistente, então não existe fórmula $V(x)$ de $L_{\text {P.A. }}$ tal que, para qualquer sentença $\alpha$ de $L_{\text {P.A. }},{ }_{\text {P.A. }} \alpha \leftrightarrow V(\lceil\alpha\rceil)$. 
O relaxamento de nossa restrição anterior (polemicamente, tarskiana) de sempre tomarmos sistemas formais como segundo e quinto elementos da estrutura tarskiana permitirá um fortalecimento desse resultado.

Definimos, agora, um sistema $S(\mathbb{N})$ tal que $\vdash_{S(\mathbb{N})} \alpha$ se e somente se $\mathbb{N} \vDash \alpha^{(19)}$; desse modo, $S(\mathbb{N})$, obviamente, não é um sistema formal no sentido em que estamos empregando o termo [cf. p. 18].

Assim, podemos partir da estrutura $\left\langle L_{\text {P.A. }}, S(\mathbb{N}), I_{\mathbb{N}}, L_{\text {P.A. }}, S(\mathbb{N}),\lceil\rceil, \Delta\right\rangle-$ ou, menos pleonástica e mais simplesmente, $\left\langle L_{\text {P.A. }}, \mathbb{N},\lceil\rceil\right\rangle$-para estabelecermos:

Indefinibilidade $\mathbb{N}$ de Gödel-Tarski. Não existe nenhuma fórmula $\mathrm{V}(x)$ de $\mathrm{L}_{\mathrm{P} . \mathrm{A}}$. tal que, para qualquer sentença $\alpha$ de $L_{P . A .},{ }_{S(\mathbb{N})} \alpha \leftrightarrow V(\lceil\alpha\rceil) .{ }^{(20)}$

Ou seja, não existe nenhuma fórmula $\mathrm{V}(x)$ de $L_{\text {P.A. }}$ tal que, para qualquer sentença $\alpha$ de $L_{P . A .}, \mathbb{N} \vDash \alpha \leftrightarrow V(\lceil\alpha\rceil)$.

Em suma, Tarski faz mais do que simplesmente construir uma definição de verdade, ele constrói uma estrutura na qual essa mesma definição faz sentido (cf. 1.1); estrutura, essa, suficientemente flexível para suportar uma série de modificações, tanto técnica quanto teoricamente, fecundas; algo que explicaria, pelo menos em parte, o sucesso da abordagem do autor.

19. Devemos notar que $\mathbb{N} \vDash \alpha$ tal como o definimos costumeiramente é um resultado das considerações de Tarski que estamos discutindo aqui e, além disso, essa definição depende, incidentalmente, de $\mathrm{I}_{\mathbb{N}}$; felizmente, devido à natureza abstrata da discussão, isso não será um problema para nós (tomamos apenas um atalho).

20. Embora o argumento empregado para estabelecer as "indefinibilidades" não seja muito diferente do aplicado por Tarski no âmbito das linguagens naturais, ele conduz, como podemos ver, a conclusões totalmente diferentes. No caso específico das linguagens naturais: podemos partir, por exemplo, da "estrutura" $\langle$ ESLP, ICLP, "〉, onde ESLP e ICLP representam, respectivamente, as expressões sintáticas da língua portuguesa e a interpretação coditiana da língua portuguesa. Agora, uma vez que é intuitivamente claro que " $x$ ' é uma sentença verdadeira" é uma expressão da língua portuguesa e, por ICLP, uma definição de verdade da "estrutura de partida", temos que 〈ESLP, ICLP, ", "“ $x$ ' é uma sentença verdadeira"〉 é um tipo generalizado de estrutura tarskiana. Ora, uma vez que várias formas do paradoxo do mentiroso podem ser construídas nessa "estrutura"; estabelecemos, assim, que 〈ESLP, ICLP, ", " “ $x$ ' é uma sentença verdadeira” ’é inconsistente (ou seja, que existem sentenças de ESLP que são tanto verdadeiras quanto falsas em ICLP). Diferentemente, no caso da Indefinibilidade $\mathbb{N}$, temos que o conjunto das sentenças válidas em $\mathbb{N}$ é consistente e, portanto, que não deverá existir nenhuma definição de verdade $\mathrm{V}(x)$ de $\mathrm{L}_{\text {P.A. }}$ em $\mathbb{N}$. 


\section{$\S 1.3$. Resultados tarskianos}

Apresentaremos, agora, alguns resultados da abordagem definicional de Tarski relativos à aritmética de primeira-ordem. Exporemos, contudo, tais resultados, tomando $\mathrm{S}=\left\langle\mathrm{L}_{\text {P.A. }}\right.$, P.A., $\left.\mathrm{I}_{\mathbb{N}}, \mathrm{L}_{\mathrm{ZF}}, \mathrm{ZF},{ }^{*}\lceil\rceil^{*}, \mathrm{t}_{\mathrm{CF}} \mid \mathrm{V}(x)\right\rangle$ [cf. p. 22] como estrutura de base, ao invés da estrutura "mais estritamente tarskiana" $\left\langle L_{\text {P.A. }}\right.$ P.A., $\left.\mathrm{I}_{\mathbb{N}}\right| L_{\text {P.A. }}^{\prime}$ P.A., $\left.\mathrm{n} t \mathrm{~V}(x)\right\rangle$-notese, entre outras coisas, as diferentes posições de 'l'.

Seja $\operatorname{SENT}(x)$ uma contrapartida formal de " $x$ é uma sentença de $L_{P . A . " ~}$ "(21):

\section{Teorema I.}

a) Para qualquer sentença $\alpha$ de $L_{\text {P.A. }},{ }{ }_{\mathrm{ZF}} t_{\mathrm{CF}}(\alpha) \leftrightarrow \mathrm{V}\left({ }^{*}\lceil\alpha\rceil^{*}\right) ;^{(22)}$

b) $\vdash_{\mathrm{ZF}} \forall x(\mathrm{~V}(x) \rightarrow \mathrm{SENT}(x))$.

Definição B. A sentença $t_{1}(\alpha) \leftrightarrow V_{1}\left(n_{1}(\alpha)\right)$ é, no contexto da estrutura $\left\langle L_{1}, A_{1}, I_{l}\right.$, $\left.\mathrm{L}_{2}, \mathrm{~A}_{2}, \mathrm{n}_{1}, \mathrm{t}_{1}, \mathrm{~V}_{1}(x)\right\rangle$, chamada bicondicional de Tarski de $\alpha{ }^{(23)}$

Assim, por a) do Teorema I, temos que todos os bicondicionais de Tarski são deriváveis em $\mathrm{ZF}$.

Seja $\dot{\sim} x$ uma contrapartida formal da função "a negação de $x$ ":

Teorema II. $\vdash_{\mathrm{ZF}} \forall x(\operatorname{SENT}(x) \rightarrow(\sim \mathrm{V}(x) \vee \sim \mathrm{V}(\dot{\sim} x)))$ [cp. Teorema 1, Tarski, 1956, p. 197].

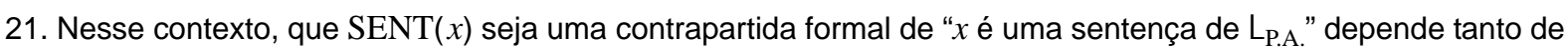
$\left.{ }^{*}\right\rceil^{*}$ quanto de ZF; pois, entre outras coisas, queremos que, para qualquer expressão $\alpha$ de $L_{\text {P.A. }}$, $\alpha$ seja uma sentença de $L_{\text {P.A. }}$ se somente se ${ }_{Z \text { ZF }} \operatorname{SENT}\left({ }^{*}[\alpha]^{*}\right)$. Os casos mais usuais são muito similares, que $\exists y(\overline{2} \cdot y=x)$ seja uma contrapartida formal de " $x$ é par" depende, analogamente, de ${ }^{-}$e $\mathbb{N}$.

22. Devemos notar que $\left.{ }^{*} \mid x\right\rceil^{*}$ e $t_{\mathrm{CF}}(x)$ são funções da meta-metateoria informal, uma vez que associam linguagem e metalinguagem, elas não são expressões de $\mathrm{L}_{\mathrm{ZF}}$, e, portanto, ' $\vdash_{\mathrm{ZF}} \forall x\left(\operatorname{SENT}(x) \rightarrow\left(\mathrm{t}_{\mathrm{CF}}(x) \leftrightarrow \mathrm{V}\left({ }^{*}\lceil x\rceil^{*}\right)\right)\right)^{\prime} \mathrm{e}$ ' ${ }_{\mathrm{ZF}} \forall x\left(\operatorname{SENT}(x) \rightarrow\left(\mathrm{t}_{\mathrm{CF}}(x) \leftrightarrow \mathrm{V}(x)\right)\right)$ ' simplesmente não fazem sentido.

23. Tarski [1935, p. 268] se refere a essas sentenças como "Teildefinitionen der Wahrheit"-"partial definitions of the truth" na tradução de Woodger [Idem, 1956, p. 155]-; entretanto, usaremos a tradução imediata dessa expressão no sentido empregado por Hajek e Pudlak [1998, pp. 28-44]. 
Teorema III. $\vdash_{\mathrm{ZF}} \forall x(\operatorname{SENT}(x) \rightarrow(\mathrm{V}(x) \vee \mathrm{V}(\dot{\sim} x)))$ [cp. Teorema 2, ibidem, p. 197].

Os teoremas II e III estabelecem, respectivamente, que os princípios de nãocontradição e do terceiro excluído para $\mathrm{V}(x)$. Enquanto, o próximo teorema estabelecerá a correção de P.A, ou seja, que os teoremas de P.A. são, como se poderia esperar, todos verdadeiros no modelo padrão da aritmética.

Seja $\operatorname{Pr}_{\text {P.A. }}(x)$ uma contrapartida formal de " $x$ é um teorema de P.A.":

Teorema IV. ${ }_{\mathrm{ZF}} \forall x\left(\operatorname{Pr}_{\text {P.A. }}(x) \rightarrow \mathrm{V}(x)\right)$ [cp. Teorema 5, ibidem, p.198].

O próximo resultado é análogo do Teorema 6 do artigo de Tarski [ibidem, p.198]; entretanto, o argumento de Tarski não pode ser transferido à estrutura $S^{(24)}$. De fato, o teorema abaixo é tanto um corolário quanto uma versão (por assim dizer, intrassistêmica) do Teorema da Incompletude de Gödel em ZF.

Teorema V. ${ }_{\mathrm{ZF}} \exists x\left(\sim \operatorname{Pr}_{\mathrm{P} . \mathrm{A} .}(x) \wedge \mathrm{V}(x)\right)$.

Não é difícil notar que as possibilidades expressivas de $\mathrm{V}(x)$ no contexto dos métodos de aritmetização de Gödel são enormes e podem conduzir a uma infinidade resultados aos quais o texto de Tarski não faz nenhuma referência.

Por exemplo, sejam $\operatorname{TM}(x), \doteq(x, y)$ e $x(y / z)$ contrapartidas formais, respectivamente, de " $x$ é um termo de LP.A.", de "a equação cujo primeiro termo é $x$ e cujo segundo é $y$ " e de "a substituição do termo $y$ pelo termo $z$ na fórmula $x$ ":

Teorema VI. $\vdash_{\mathrm{ZF}} \forall x \forall y \forall z((\mathrm{~V}(x) \wedge \mathrm{TM}(y) \wedge \mathrm{V}(\dot{=}(y, z))) \rightarrow \mathrm{V}(x(y / z)))$.

24. A estrutura de partida do artigo de Tarski [cf. 1956, pp.168-169] é, essencialmente, $\left\langle L_{\subseteq}, A_{\subseteq}, I_{c}\right\rangle$, onde $L_{\subseteq}=\{\subseteq\}$, $\operatorname{Dom}\left(\mathrm{I}_{\mathrm{C}}\right)=$ o conjunto potência de $\mathrm{C}, \mathrm{A}_{\subseteq}(\subseteq)=$ a relação "é subconjunto de". Assim, uma vez que a cardinalidade de $C$ não é especificada por nenhum axioma e que $\forall x \forall y(x \subseteq y)$ é válida se Cé vazio, enquanto $\sim x \forall y(x \subseteq y)$ é válida se $C$ não é vazio; temos, pela correção de $\mathrm{A}_{\subseteq}$, que nem $\forall x \forall y(x \subseteq y)$ nem $\sim \forall \forall y(x \subseteq y)$ são deriváveis em $\mathrm{A}_{\subseteq}$. Contudo, qualquer modelo de $\mathrm{A}_{\subseteq}$ deverá verificar uma das duas sentenças, de modo que ou temos $\mathrm{V}(\mathrm{n}(\forall x \forall y(x \subseteq y)))$ ou temos $\mathrm{V}(\mathrm{n}(\sim \forall x \forall y(x \subseteq y)))$ e, portanto, o Teorema 6 de Tarski. 


\section{§2. Axiomáticas da verdade}

\section{§2.1. Da abordagem definicional à axiomática}

A construção tarskiana da estrutura de chegada é, de fato, bastante natural e direta; suas etapas são, em suma, as seguintes:

a) Incorporação das expressões da linguagem-objeto como termos da metalinguagem [Tarski, 1956, pp. 170-171];

b) Regulamentação das propriedades dos termos por meio de axiomas metateóricos de caráter combinatório [ibidem, pp. 173-174];

c) E, finalmente, adição de ferramentas teóricas capazes de controlar a definição das sequências de satisfação ${ }^{(25)}$ [no caso da metalinguagem, cf. ibidem, p. 171; no caso da metateoria, cf. ibidem p. 173]. ${ }^{(26)}$

Esse caráter direto da construção de Tarski e os resultados da seção anterior são alguns dos pontos fortes da abordagem definicional de Tarski e, como tais, podem ser, e reiteradamente são, mobilizados como testemunhas de sua adequação.

Não obstante, uma alternativa às abordagens definicionais em geral e às estruturas tarskianas em particular é a introdução axiomática do predicado de verdade. Existem, como seria de se esperar, diversas formas de justificar essa abordagem; a mais direta delas é, ao que parece, sustentar que a verdade é, em si mesmo, um conceito primitivo e que, portanto, não faria muito sentido analisá-la em termos de conceitos mais fundamentais; desse modo nossos axiomas poderiam ser diretamente justificados por nossas intuições mais fundamentais sobre a verdade ${ }^{(27)}$.

25. A construção de uma estrutura tarskiana, cuja estrutura de partida é, por exemplo, $\langle$ L, A, I $\rangle$, é uma tarefa razoavelmente complexa; devemos, entre outras coisas, associar cada uma das fórmulas de $\mathrm{L}$ com uma relação entre certos "objetos", eles próprios, construídos a partir do domínio de I ; as sequências de satisfação de Tarski são simplesmente sequências categorialmente adequadas desses "objetos".

26. A metateoria deverá, segundo Tarski [1956, p.173] incluir "axiomas gerais da lógica" em número suficiente para tanto. Devemos notar que princípios de ordem superior (em ultima instância, de ordem tanto finita quanto infinita) são admitidos por Tarski como "axiomas gerais da lógica". 
Não pretendemos, contudo, elaborar nesse estudo uma justificação articulada e última da abordagem axiomática.

Apresentaremos abaixo, isso sim, duas motivações de ordem, por assim dizer, estratégica e metodológica:

a) A primeira será sustentada por uma analogia e remete às futuras possibilidades das teorias axiomáticas, é essa a motivação que, apesar de ser aparentemente mais frágil, deve ser tomada como oficial;

b) A segunda apelará para certa parcimônia na utilização de ferramentas lógicomatemáticas (quantificação, conjuntos, etc.) que são necessárias ao tratamento definicional rigoroso do predicado de verdade. ${ }^{(28)}$

Utilizaremos dois tipos de modificações das estruturas tarskianas com vistas à primeira motivação.

Em primeiro lugar, a determinação mais completa das estruturas de partida permite estabelecer resultados esclarecedores sobre as relações recíprocas existentes entre os mais diversos sistemas. Por exemplo, enunciamos, anteriormente, os teoremas da Definibilidade $\left\langle\right.$ P.A., $\left.\mathrm{I}_{\mathbb{N}}, \mathrm{ZF}\right\rangle$ e da Indefinibilidade $\mathbb{N}$ [cf. pp. 22-23]. Ora, tais teoremas sugerem, entre outras coisas, que $\mathbb{N}$ e ZF estão relacionados de um modo (o predicado de verdade de $\mathrm{I}_{\mathbb{N}}$ é definível em $\mathrm{ZF}$ ) que $\mathbb{N}$ não está relacionado consigo mesmo.

27. Um partidário desse ponto de vista é Donald Davidson em 'The Folly of Trying to Define Truth', [2005, pp.2021]: "For the most part, the concepts philosophers single out for attention, like truth [...], are the most elementary concepts we have, concepts without which [...] we would have concepts at all. Why then should expect to be able to reduce these concepts definitionally to other concepts that are simpler, clearer, and more basic? [...] [W]e cannot hope to underpin [the concept of truth] with something more transparent or easier to grasp.".

28. Existe uma distinção entre justificações ou motivações intrínsecas e extrínsecas para a adoção de novos axiomas [cf. discussão de Penelope Maddy em Defending The Axioms, 2011, notadamente, pp. 123-137]; e, embora não estejamos nesse momento discutindo motivações para aceitação de determinados axiomas, mas do próprio método axiomático em oposição à abordagem definicional de Tarski, um pequeno abuso da linguagem talvez seja desculpável. A defesa (justificação) de Davidson do método axiomático (no tratamento do predicado de verdade) seria, então, intrínseca: a verdade é um conceito primitivo e deve ser tratado como tal [nota 26, p. 11]. Nossa justificação oficial seria extrínsica (e também vaga): remete a "futuras possibilidades". O recurso a "certa parcimônia" seria, por sua vez, ambíguo: intrínseco, para aqueles que recusam a legitimidade das ferramentas metateóricas de Tarski; extrínseco, para aqueles que querem apenas estudar as relações entre as estruturas tarskianas e certas teorias axiomáticas. 
Desse modo, algumas questões surgem naturalmente:

a) Outros sistemas estão relacionados do mesmo modo que $\mathbb{N}$ e ZF? Quais?

b) A relação de definibilidade é irreflexiva? É transitiva?

Em segundo lugar, restrições impostas à linguagem da estrutura de partida permitem estabelecer resultados, também eles esclarecedores, sobre as relações existentes entre certos fragmentos de uma linguagem dada e, consequentemente, sobre as capacidades expressivas desses fragmentos. Seja, por exemplo, $L_{0}$ um fragmento de $L_{P . A .}$ no qual não há ocorrência de quantificadores; nesse caso específico, é possível construir uma definição de verdade tomando $\left\langle\mathrm{L}_{0}\right.$, P.A., $\mathrm{I}_{\mathbb{N}}$, L.A., P.A., $\left.\rceil, \Delta\right\rangle$ como estrutura de partida (obviamente, o teorema da definibilidade será, nesses casos, nossa pedra de toque).

$\mathrm{E}$, assim, surgem novas questões:

a) Existem sublinguagens $L_{1}$ de $L_{P . A}$ mais inclusivas do que $L_{0}$ tais que uma definição de verdade possa nelas ser construída (a estrutura de partida seria nesse caso $\left\langle L_{1}\right.$, P.A., $\mathrm{I}_{\mathbb{N}}, L_{P . A .}$, P.A., \lceil\rceil$\left.\left., \Delta\right\rangle\right)$ ? Se existem, quais?

b) Existem sublinguagens próprias $L_{2}$ de $L_{P . A}$ que permitem definições de verdade (a estrutura de partida seria $\left\langle\mathrm{L}_{0}\right.$, P.A., $\mathrm{I}_{\mathbb{N}}, \mathrm{L}_{2}$, P.A., \lceil\rceil$\left.\left., \Delta\right\rangle\right)$ ?

Devemos notar, então, que não há incoerência nenhuma entre a) aceitarmos a construção tarskiana como abordagem correta e adequada do predicado de verdade e b) exploramos todo tipo de modificação das estruturas envolvidas. Além disso, essas modificações, como tentamos mostrar por meio dos exemplos acima, são extremamente fecundas do ponto de vista teórico. Assim, uma vez que não temos ainda os subsídios necessários para julgar as abordagens axiomáticas do predicado de verdade; podemos, no mesmo espírito dedicado às modificações estruturais, explorar-Ihes as possibilidades teóricas. Essa será, por assim dizer, a posição oficial aceita nesse estudo. 
Podemos, não obstante, ir além desse expediente de caráter meramente analógico. Devemos notar, para tanto, que as ferramentas necessárias ao controle das sequências de satisfação estão longe de ser triviais. A aritmética elementar, por exemplo, deve necessariamente estar incluída na metateoria do cálculo de classes do artigo de Tarski; pois, do contrário, não seríamos capazes de lidar, de maneira uniforme, com funções sentencias de uma aridade qualquer [cf. Tarski, 1956, pp. 191-192].

E, embora seja verdade que, nesse caso específico, possamos replicar que isso não é um grande problema, uma vez que a aritmética elementar já está implícita no tratamento metateórico dos nomes descritivos-estruturais [cf. p. 16 e Tarski, 1956, p. 173], no caso de teorias mais fortes que aquela apresentada como exemplo no artigo de Tarski (e isso vale praticamente para qualquer teoria matematicamente relevante), as demandas podem não ser aceitas de modo tão pacífico.

No caso da aritmética elementar, por exemplo, a construção estritamente tarskiana relativa à estrutura de partida $\left\langle L_{P . A .}\right.$ P.A., $\left.I_{\mathbb{N}}\right\rangle$ leva à quantificação de propriedades ${ }^{(29)}$; e alguns lógicos (e.g., Quine) poderiam não aceitar a demanda.

No caso da teoria dos conjuntos, tudo fica pior. Se tomássemos, por exemplo, ZF como ponto de partida, encontraríamos, eventualmente, estruturas metateóricas nãoconjuntistas no ponto de chegada. Desse modo, estaríamos, dizem alguns, fora do domínio da matemática ordinária e, portanto, para um bom número desses autores, além de qualquer demanda teórica aceitável.

Nesse sentido, uma abordagem axiomática nos permite ser matematicamente mais parcimoniosos. Pois, ao invés de incorporarmos todas ferramentas matemáticas necessárias à definição da metalinguagem e da metateoria tarskianas, poderíamos introduzir o conceito de verdade como um predicado primitivo ${ }^{(30)}$; o que, nos casos de P.A.

29. De fato, uma metateoria adequada para $\left\langle L_{P . A .}\right.$, P.A., $\left.I_{\mathbb{N}}\right\rangle$ demanda uma teoria dos subconjuntos de números naturais. É, por isso, que tanto a tarskiana P.A'. quanto ZF podem podem cumprir o papel de metateoria de P.A.. 
e ZF, pode ser feito diretamente pela incorporação do predicado de verdade à linguagemobjeto, uma vez que as traduções entre expressões e termos são passíveis, por meio dos métodos de aritmetização gödelianos, de tratamento nas próprias teorias.

Esse é o caminho que trilharemos nesse estudo.

\section{§2.2. Algumas teorias axiomáticas da verdade}

Obviamente, aceitar a abordagem axiomática, mesmo que em termos quase meramente estratégicos, é apenas o primeiro passo; devemos lidar com uma questão crucial: Qual conjunto de axiomas, dentre os vários existentes, devemos subscrever? Para que tenhamos apenas uma vaga ideia do problema, um livro recente sobre teorias axiomáticas da verdade introduz nada menos que 12 famílias de teorias ${ }^{(31)}$ (algumas delas contando com um número infinito de teorias). De modo nada surpreendente, uma vez que tais teorias não são em geral introduzidas sem alguma motivação, uma quantidade enorme de argumentos (entre justificações intrínsecas e extrínsecas), tanto de caráter mais estritamente lógico, quanto mais amplamente filosófico, pode ser mobilizada em nome da adequação de uma ou outra das alternativas.

Entretanto, nosso objetivo aqui não será a análise de tais argumentos; gostaríamos, apenas, de introduzir quatro diferentes teorias "aparentadas" duas a duas, tanto como preâmbulo quanto como termos de comparação para as axiomáticas que iremos propor no decorrer desse estudo:

a) A primeira família de teorias será baseada nos bicondicionais de Tarski [cf. p. 24].

b) A segunda será baseada nas cláusulas recursivas empregadas na construção das metateorias tarskianas.

[cf. ibidem, p. 255 e segs.]. Contudo, como o autor nota no Postscript de seu artigo, essa suposta impossibilidade pode ser superada pelo uso de ordinais transfinitos [cf. ibidem, p. 268 e segs.]. Devemos notar que nem o caráter negativo do julgamento de Tarski nem a emenda conseguem afastar o fato de que Tarski é pioneiro tanto da abordagem definicional quanto da abordagem axiomática do predicado de verdade.

31. Axiomatic Theories of Truth de Volker Halbach, 2011. 
Definimos, primeiramente, a linguagem $L_{\text {P.A.V. }}$ pela adição do predicado $\mathrm{V}$ como uma nova constante em $L_{P . A}$ Segue-se, então, a primeira de nossas duas famílias.

Definição C. A teoria BT(V) em L $_{\text {P.A.V. }}$ será definida pelo seguinte conjunto de axiomas:

a) Os axiomas da teoria Q de Robinson;

b) As instâncias do esquema de indução matemática restrito às fórmulas de $L_{P . A}$;

c) Os bicondicionais de Tarski restritos às sentenças de $L_{P . A}$ :

Definiremos, além disso, uma variante bem simples de BT(V):

Definição D. A teoria BTV em $L_{P . A . V . ~}$ será definida pelo seguinte conjunto de axiomas:

a) Os axiomas da teoria Q de Robinson;

b) As instâncias do esquema de indução (em L L.A.V.);

c) Os bicondicionais de Tarski restritos às sentenças de $L_{P . A:}{ }^{(32)}$

A diferença entre as teorias está na aceitação ou não das instâncias do esquema da indução nas quais a "nova" constante V aparece. Devemos notar que não há nada de especial em aceitar essas instâncias; na verdade, é muito natural que as aceitemos, uma vez que a indução é intuitivamente válida para quaisquer subconjuntos de números naturais e que a extensão de V no modelo padrão da aritmética é, claramente, um subconjunto dos números naturais. ${ }^{(33)}$

32. É possível provar que se P.A. é consistente, então BT(V) e BTV também o são. Seja $\delta=\left\langle\alpha_{1}, \alpha_{2}, \ldots, \alpha_{n}, \alpha\right\rangle$ uma derivação em BTV (ou em BT(V)), sejam $\mathrm{V}\left(\left\lceil\varphi_{1}\right\rceil\right) \leftrightarrow \varphi_{1}, \ldots, \mathrm{V}\left(\left\lceil\varphi_{i}\right\rceil\right) \leftrightarrow \varphi_{i}, i \leqslant n_{+}$, os bicondicionais de Tarski de $\delta$. Definimos $\mathbb{X}\left(\alpha_{j}\right)$ como a substituição de cada subfórmula $V(\tau)$ de $\alpha_{j}$ pela fórmula $\left(\tau=\left\lceil\varphi_{1}\right\rceil \wedge \varphi_{1}\right) \vee \cdots \vee$ $\left(\tau=\left\lceil\varphi_{i}\right\rceil \wedge \varphi_{i}\right)$. Temos, então, que se $\alpha_{k}$ é um axioma de BTV, então $\vdash_{\text {P.A. }} \mathbb{X}\left(\alpha_{k}\right)$ [base]. Seja $\vdash_{\text {P.A. }} \mathbb{W}\left(\alpha_{1}\right)$, $\vdash_{\text {P.A. }} \mathbb{W}\left(\alpha_{2}\right), \ldots, r_{\text {P.A. }} \mathbb{W}\left(\alpha_{n}\right)$ [hipótese da indução], segue-se, uma vez que ou (1) $\alpha$ é um axioma ou (2) $\alpha=\forall v \alpha_{l}$ ou (3) $\alpha=\alpha_{l} \rightarrow \alpha$, para algum $l \leqslant n$, e que (4) $\mathbb{W}\left(\forall v \alpha_{l}\right)=\forall v \mathbb{W}\left(\alpha_{l}\right)$ e (5) $\mathbb{W}\left(\alpha_{l} \rightarrow \alpha\right)=\mathbb{W}\left(\alpha_{l}\right) \rightarrow \mathbb{W}(\alpha)$, que $(6)$ P.A. $_{\text {P }}(\alpha)$ (não é difícil notar que existem versões construtivas da prova). Assim, uma vez que, para toda fórmula $\beta$ de $L_{P . A}$, $X(\beta)=\beta$, qualquer fórmula de $L_{P . A}$ derivável em BTV também é derivável em P.A.; segue-se como corolário que se P.A. é consistente, então BT(V) e BTV também o são.

33. Esse é basicamente o argumento que Feferman apresenta para justificar o que ele chama "unfolding of a system" [cf., por exemplo, "Gödel's program for new axiomas: Why, where, how and what?”, pp. 8-10]. 
Bem outro, entretanto, é caso da restrição dos bicondicionais de Tarski às sentenças de L.A. Seja BTV* uma versão de BTV (o argumento é válido mutatis mutandis também para BT(V)) na qual a restrição é relaxada; podemos, então, mostrar que $\mathrm{BTV}^{*}$ é inconsistente por meio do seguinte argumento:

a) O Lema do Ponto-fixo é válido em $\mathrm{BTV}^{*}$; desse modo, existirá uma sentença $\varphi$ de $L_{\text {P.A.V. }}$ (mas não de $L_{\text {P.A. }}$ se P.A. for consistente!) tal que $\vdash_{\mathrm{BTV}^{*}} \varphi \leftrightarrow \sim \mathrm{V}(\lceil\varphi \mid)$ ). Ora, desde que b) os bicondicionais de Tarski são válidos para quaisquer sentenças de L.A.V. $_{\text {. }}$ temos que c) $\vdash_{\mathrm{BTV}^{*}} \varphi \leftrightarrow \mathrm{V}(\lceil\varphi\rceil)$ e, portanto, que d) $\vdash_{\mathrm{BTV}^{*}} \mathrm{~V}(\lceil\varphi\rceil) \leftrightarrow \sim \mathrm{V}(\lceil\varphi\rceil){ }^{(34)}$

Obviamente, 'BT' e os parênteses em '(V)' são referências, respectivamente, aos bicondicionais de Tarski e à restrição imposta ao esquema de indução matemática.

Com vistas à definição da segunda família de teorias, teremos de introduzir uma série de símbolos auxiliares:

a) $\dot{\lambda}(x, y)$ será uma contrapartida formal da função "a conjunção entre $x$ e $y$ ", ou seja, da função que atribui, aos números de Gödel de duas fórmulas dadas, o número de Gödel da conjunção dessas fórmulas;

b) $\dot{\vee}(x, y), \dot{\rightarrow}(x, y), \dot{\forall}(x, y)$ e $\dot{\exists}(x, y)$ serão, respectivamente,contrapartidas formais das funções "a disjunção entre $x$ e $y$ ", "a condicionalização de $y$ por $x$ ", "a universalização de $y$ com respeito a $x$ ", "a existencialização de $y$ com respeito a $x$ ".

34. Poderíamos, apesar de tudo, defender formas não-restritas dos bicondicionais de Tarski; no caso, deveríamos derrogar alguns dos princípios da lógica clássica, seja para evitar diretamente as contradições, seja para evitar que elas trivializem as teorias correpondentes [cf., por exemplo, Field, 2008, pp. 4-11]. E, embora a defesa de formas não-restritas dos bicondicionais de Tarski se dê, geralmente, no nível informal do predicado de verdade da linguagem natural [cf., por exemplo, Priest, 2006a, pp. 15 e segs.], isso não nos impede de transferi-la à aritmética formal. Uma questão, no mínimo interessante, seria aquela do status das sentenças nas quais o predicado de verdade ocorre (regulado por formas não-restritas de bicondicionais) em aritméticas desenvolvidas em sistemas de lógica paraconsistente. Suponhamos que seja possível definir uma aritmética aceitável em um desses sistemas, podemos então formular as seguintes perguntas: Para quais sentenças, se para alguma, podemos provar que a não-contradição vale? Para quais sentenças, se para alguma, podemos provar que a não-contradição falha? Existiria alguma relação entre a ocorrência ou não do predicado de verdade e esses tipos de sentenças? 
c) $\operatorname{LTF}(x)$ será, por sua vez, uma contrapartida formal da função "a denotação (valor ou legenda) do termo fechado x", ou seja, da função que atribui, ao número de Gödel de um termo fechado da linguagem, o número ao qual esse termo se refere; seja, por exemplo, $n$ o número de Gödel do termo $(\overline{2}+\overline{2}) \cdot \overline{2}$, a legenda de $n$ será, portanto, 8 .

d) $\mathrm{E}$, finalmente, $\mathrm{TF}(x)$ será uma contrapartida formal do predicado " $x$ é um termo fechado de L.A.". $_{\text {. }}$

Definição E. As sentenças listadas abaixo ${ }^{(35)}$ serão coletivamente chamadas axiomas composicionais em $\mathrm{L}_{\mathrm{P} . \mathrm{A}}$ :

a) $\forall x \forall y(\operatorname{SENT}(\dot{=}(x, y)) \rightarrow(\mathrm{V}(\dot{\doteq}(x, y)) \leftrightarrow \operatorname{LTF}(x)=\operatorname{LTF}(y))) ; \quad\left[\mathrm{C}_{=}\right]$

b) $\forall x(\operatorname{SENT}(x) \rightarrow(\mathrm{V}(\dot{\sim} x) \leftrightarrow \sim \mathrm{V}(x)))$;

c) $\forall x \forall y(\operatorname{SENT}(\dot{\lambda}(x, y)) \rightarrow(\mathrm{V}(\dot{\lambda}(x, y)) \leftrightarrow \mathrm{V}(x) \wedge \mathrm{V}(y)))$;

d) $\forall x \forall y(\operatorname{SENT}(\dot{\vee}(x, y)) \rightarrow(\mathrm{V}(\dot{\vee}(x, y)) \leftrightarrow \mathrm{V}(x) \vee \mathrm{V}(y)))$;

e) $\forall x \forall y(\operatorname{SENT}(\dot{\rightarrow}(x, y)) \rightarrow(\mathrm{V}(\dot{\rightarrow}(x, y)) \leftrightarrow \mathrm{V}(x) \rightarrow \mathrm{V}(y)))$;

f) $\forall x \forall y(\operatorname{SENT}(\dot{\forall}(x, y)) \rightarrow(\mathrm{V}(\dot{\forall}(x, y)) \leftrightarrow \forall z(\operatorname{TF}(z) \rightarrow \mathrm{V}(y(x / z))))) ;{ }^{(36)}$

g) $\forall x \forall y(\operatorname{SENT}(\dot{\exists}(x, y)) \rightarrow(\mathrm{V}(\dot{\exists}(x, y)) \leftrightarrow \exists z(\operatorname{TF}(z) \wedge \mathrm{V}(y(x / z))))$.

Definição F. A teoria $C(V)$ em $L_{\text {P.A.V. }}$ será definida pelo seguinte conjunto de axiomas:

a) Os axiomas da teoria $\mathrm{Q}$ de Robinson;

b) As instâncias do esquema de indução restrito às fórmulas de $L_{P . A}$;

c) Os axiomas composicionais em $L_{P . A}$ :

Definição G. A teoria CV em $L_{P . A . V .}$ será definida pelo seguinte conjunto de axiomas:

a) Os axiomas da teoria Q de Robinson;

35. Os axiomas composicionais podem ser pensados como contrapartidas intrassistêmicas das cláusulas recursivas da definição de verdade tarskiana. Temos, assim, que a base da definição de Tarski é representada por $\mathrm{C}_{=}$enquanto as cláusulas indutivas são respresentadas pelos demais axiomas [cf. 1956, p. 193, df. 22].

36. Sobre a fórmula $x(y / z)$, cf. p. 25, Teorema VI. 
b) As instâncias do esquema de indução (em L $L_{\text {P.A.V. }}$ );

c) Os axiomas composicionais em LP.A:

Obviamente, 'C' é uma referência aos axiomas composicionais, de modo $\mathrm{C}(\mathrm{V})$ é, por assim dizer, uma teoria composicional restrita, enquanto CV é uma teoria composicional ampla da verdade.

\section{§2.3. Demandas Tarskianas}

O objetivo desse estudo, deixemos claro, não é estabelecer que um dos sistemas acima (ou qualquer outro) captura de maneira adequada e absoluta as leis que regulam o predicado de verdade. De fato, há um ceticismo bem fundamentado quanto a essa possibilidade. Devemos, isso sim e mais frouxamente, notar que todos os axiomas propostos anteriomente são intuitivamente válidos ${ }^{(37)} \mathrm{ou}$, em outras palavras, que os sistemas da seção anterior são todos corretos de um ponto de vista, por assim dizer, lógico-intuitivo ${ }^{(38)}$. No que se segue, introduziremos alguns resultados relativos aos sistemas propostos na seção anterior com vistas a compará-los tanto entre si quanto em relação à teoria definicional tarskiana.

Lema VII. BT(V) é um subsistema de BTV, BTV é um subsistema de C(V) [Halbach, 2011, p. 66] e C(V) é um subsistema de CV.

37. Ou seja, nossa justificação dos axiomas, desse ponto de vista, seria intrínseca [cf. nota 28, p. 27] (desde que aceitemos os resultados de Tarski como guias fidedignos). Contudo, o problema aqui é outro: até onde devemos ir? Devemos ou não aceitar tudo que é intuitivamente válido? Nossa resposta é: analisaremos vários desses sistemas (mais ou menos inclusivos) com vistas a estabelecermos suas características e a compará-los; tudo isso em nome da fecundidade própria do método axiomático, deixando, estrategicamente, de lado a pretensão de estabelecer a "adequação" absoluta de qualquer um desses sistemas.

38. Lógico-intuitivo em oposição a um sentido mais propriamente lógico que demanda a apresentação de um modelo. Notemos algumas diferenças: a derivação de uma contradição em um sistema intuitivamente correto conduziria à revisão do próprio conteúdo intuitivo do predicado e/ou da lógica subjacente ao sistema. No segundo caso, os sistemas deverão ser ou não corretos em relação ao modelo dado, sendo que da correção se segue necessariamente que nenhuma contradição é derivável no sistema (qualquer inconsistência aqui é uma inconsistência da teoria dos modelos não do modelo ele mesmo). 
Lema VIII. Os sistemas BT(V), BTV e C(V) são todos conservativos sobre P.A. [ibidem, p. 55 e p. 80].

Temos, ainda, que:

Teorema IX. $\forall x(\operatorname{SENT}(x) \rightarrow(\sim \mathrm{V}(x) \vee \sim \mathrm{V}(\dot{\sim} x)))$ não é derivável em $\mathrm{C}(\mathrm{V})$.

Enquanto que:

Teorema $\mathbf{X} . \vdash_{\mathrm{CV}} \forall x(\operatorname{SENT}(x) \rightarrow(\sim \mathrm{V}(x) \vee \sim \mathrm{V}(\dot{\sim} x)))$.

Analogamente, temos que:

Teorema XI. $\forall x(\operatorname{SENT}(x) \rightarrow(\mathrm{V}(x) \vee \mathrm{V}(\dot{\sim} x)))$ não é derivável em $\mathrm{C}(\mathrm{V})$.

Teorema XII. $\vdash_{\mathrm{CV}} \forall x(\operatorname{SENT}(x) \rightarrow(\mathrm{V}(x) \vee \mathrm{V}(\dot{\sim} x)))$.

Em suma, CV é o único dos sistemas propostos no qual podemos estabeler os princípios de não-contradição e terceiro excluído. O caso da correção de P.A. não é diferente:

Teorema XIII. $\forall x\left(\left(\operatorname{SENT}(x) \wedge \operatorname{Pr}_{\mathrm{P} . \mathrm{A} .}(x)\right) \rightarrow \mathrm{V}(x)\right)$ não é derivável em $\mathrm{C}(\mathrm{V})$.

Teorema XIV. ${ }_{\mathrm{CV}} \forall x\left(\left(\operatorname{SENT}(x) \wedge \operatorname{Pr}_{\mathrm{P} . \mathrm{A} .}(x)\right) \rightarrow \mathrm{V}(x)\right)$ [ibidem, p. 104].

Do Teorema XIV e do teorema da incompletude para P.A., temos que:

Teorema XV. CV não é conservativo sobre P.A. [ibidem, p. 106].

Está claro que CV é a única teoria proposta cuja força probatória é similar à da metateoria tarskiana. Contudo,desde que CV não é conservativo sobre P.A. e, portanto, introduz, por meio de considerações "semânticas" sobre o predicado de verdade, algumas consequências puramente aritméticas em P.A., alguns filósofos "mais modestos" em relação ao papel teórico do predicado de verdade-Künne [2003, pp. 317 e segs.] os denomina minimalistas-argumentariam que CV não é, justamente por isso, uma boa teoria da verdade. Segundo Wolfgang Künne [ibidem, p. 321]: "[...] the minimalist 
declares: Si tacuisses, philosophus mansisses, or in terms more easily comprehensible to non-classicists, silence is golden". No caso específico dos minimalistas (na expressão de Künne) ou dos deflacionistas ${ }^{(39)}$, talvez o velho e bom português seja ainda mais certeiro: "Em boca fechada não entra mosca".

\section{§2.4. Um argumento matemático e algumas alternativas teóricas}

Grande parte das ferramentas semânticas de Tarski (mas, obviamente, devido à Indefinibilidade $\mathbb{N}$ [p. 23], não todas) pode ser devidamente formalizada em sistemas como P.A. por meio dos métodos gödelianos de aritmetização.

Podemos, entre outras coisas, construir as chamadas definições parciais de verdade $^{(40)}$ ou DPVs, que formam uma lista enumerável $\mathrm{V}_{0}(x), \mathrm{V}_{1}(x), \ldots, \mathrm{V}_{n}(x), \ldots$ de fórmulas cuja única variável livre é $x$ e, para as quais, o seguinte metateorema vale:

Teorema XVI. Para qualquer número natural $n$ dado, se $\alpha$ é uma sentença de $\mathrm{L}_{\text {P.A. }}$ cuja complexidade é menor ou igual a $n$, então $\vdash_{\text {P.A. }} \alpha \leftrightarrow \mathrm{V}_{n}(\lceil\alpha\rceil)$.

Ora, cada uma das sentenças de $L_{\text {P.A. }}$ tem uma única complexidade dada, de modo que a união das definições parciais de verdade de P.A. seria um bom candidato a predicado de verdade de P.A.. Como veremos, arregimentar tal intuição e obter algumas de suas consequências será, colocando tudo em pratos limpos, o objetivo desse estudo.

Uma proposta ao mesmo tempo simples e descuidada é o acréscimo da pseudodefinição $\exists y\left(\mathrm{~V}_{y}(x)\right) \leftrightarrow \mathrm{V}(x)$ aos axiomas de P.A.. Contudo, $\exists y\left(\mathrm{~V}_{y}(x)\right) \leftrightarrow \mathrm{V}(x)$ não é nem mesmo uma fórmula de $L_{P . A . V ;}$; e estaríamos, no caso, confundindo as variáveis

39. O termo 'deflacionismo' seria, nesse caso, mais adequado, porque é tomado pela maioria dos filósofos numa acepção mais geral (e, possivelmente, mais vaga) [cf., por exemplo, Burgess, 2004, pp. 37-55], enquanto 'minimalismo' se aplica mais diretamentamente à abordagem do predicado de verdade proposta por Paul Horwich [cf. 2004, pp. 57-73].

40. Esses predicados são descritos por Hájek \& Pudlák [1998, pp. 51-61] e são usados pelos autores para estabelecer que certos fragmentos de P.A. definidos pela restrição da esquema de indução às fórmulas de uma determinada complexidade aritmética são finitamente axiomatizáveis [ibidem, pp. 77-81]. 
metalinguísticas das definições parciais de verdade com variáveis de L.A.V. Na verdade, uma das consequências da Indefinibilidade $\mathbb{N}$ é que não existe nenhuma fórmula (com duas variáveis livres) que, de alguma maneira, descreva o comportamento da sequência de definições parciais de verdade. ${ }^{(41)}$

Obviamente, embora as versões parametrizadas $\mathrm{V}_{n}(x) \leftrightarrow \mathrm{V}(x)$ da pseudodefinição sejam sentenças de L como axiomas, pelo simples fato de que é claramente falso que $\mathrm{V}(\lceil\alpha\rceil) \rightarrow \mathrm{V}_{n}(\lceil\alpha\rceil)$ no caso de sentenças de complexidade maior que $n$.

Vamos às propostas iniciais.

\section{A. Sistemas de acumulação (simples)}

Definição H. As sentenças $\forall x\left(\mathrm{~V}_{n}(x) \rightarrow \mathrm{V}(x)\right)$, onde $n$ é um número natural, serão chamadas cláusulas de acumulação positiva ou, mais sucintamente, CAPs.

Nossa primeira proposta será:

Definição I. A teoria $\mathrm{AcM}(\mathrm{V})$ em L L.A.V. será definida pelo seguinte conjunto de axiomas:

a) Os axiomas da teoria Q de Robinson;

b) As instâncias do esquema de indução matemática restrito às fórmulas de $L_{P . A}$;

c) As cláusulas de acumulação positiva. ${ }^{(42)}$

Definiremos, como fizemos anteriormente para BT(V) e C(V), uma variante de $\operatorname{AcM}(\mathrm{V})$ na qual a indução matemática não estará restrita às fórmulas de $L_{P . A}$.

41. Para uma descrição mais rigorosa do problema e uma apresentação do argumento cf., por exemplo, Boolos \& Jeffrey [1980, p. 207].

42. Esse tipo de axiomática, na qual adicionamos um número infinito de axiomas, por assim dizer, um a um, não é algo estranho na literatura. Os exemplos mais comuns são as versões de primeira-ordem das teorias de estruturas algébricas, nas quais acrescentamos um axioma de existência para cada número natural dado, impondo, assim, que o domínio da estrutura seja infinito; e. g., a teoria dos grupos infinitos tal como exposta por Epstein [2006, p. 197]. 
Definição J. A teoria AcMV em L L.A.V. será definida pelo seguinte conjunto de axiomas:

a) Os axiomas da teoria Q de Robinson;

b) As instâncias do esquema de indução (em L L.A.V.);

c) As cláusulas de acumulação positiva.

Os sistemas AcM(V) e AcMV são, pelo menos, intuitivamente tão corretos quanto BT(V), BTV, C(V) e CV. Sem entrarmos em considerações lógico-filosóficas sobre como um predicado de verdade deveria se comportar, parece matematicamente imediato que, na medida em temos acesso às definições parciais de verdade, um caminho natural é, de alguma forma, acumulá-las. Ora, desde que não podemos acumulá-las usando uma relação que as enumere, parece legítimo adicionar as cláusulas de acumulação tal como fizemos. Por esses motivos, AcM(V) e AcMV serão objetos privilegiados de nosso estudo, nossas teorias "minimais" da verdade; $\mathrm{AcM}(\mathrm{V})$ é chamado sistema minimal (restrito) da acumulação positiva; AcMV é chamado sistema minimal da acumulação positiva.

Além disso, introduziremos, com vistas ao fortalecimento de nossos sistemas minimais, certas definições parciais modificadas de verdade que chamaremos, respectivamente, definições parciais enraizadas de verdade (RVs) e definições parciais estruturais de verdade (EVs); e apresentaremos, então, cláusulas, análogas às CAPs, e sistemas de acumulação, análogos ao sistema $\operatorname{AcM}(\mathrm{V})$, baseados nessas versões modificadas das definições parciais de verdade.

\section{B. Sistemas de dupla acumulação}

Contudo, como se pode ver, tais sistemas estão longe de dar conta da noção de verdade como a união das verdades parciais. Até o momento, "acumulamos" a verdade. Podemos, ainda, dizer que a verdade é tão-somente aquilo que foi acumulado. Nem é preciso lembrar que, no caso dos sistemas axiomáticos, não temos as ferramentas da 
teoria dos conjuntos necessárias para definir a extensão de $\mathrm{V}(x)$ como a menor extensão acumulada pelas definições parciais. Iremos propor, então, dois pequenos truques.

Primeiramente, podemos também construir definições parciais de falsidade, ou seja, uma lista enumerável $\mathrm{F}_{0}(x), \mathrm{F}_{1}(x), \ldots, \mathrm{F}_{n}(x), \ldots$ de fórmulas cuja única variável livre é $x$ e, para as quais, o seguinte metateorema vale:

Teorema XVII. Para qualquer número natural $n$ dado, se $\alpha$ é uma sentença de $\mathrm{L}_{\text {P.A. }}$ cuja complexidade é menor ou igual a $n$, então $\vdash_{\text {P.A. }} \sim \alpha \leftrightarrow \mathrm{F}_{n}(\lceil\alpha\rceil)$.

E, assim, analogamente aos sistemas $\operatorname{AcM}(\mathrm{V})$ e AcMV de acumulação da verdade, poderemos definir sistemas $\mathrm{AcM}(\mathrm{F})$ e AcMF de "acumulação da falsidade" baseados nas sentenças $\forall x\left(\mathrm{~F}_{n}(x) \rightarrow \mathrm{F}(x)\right)$, chamadas cláusulas de acumulação negativa de $L_{\text {P.A.F. }}$ ou, mais sucintamente, CANs.

Podemos, então, lançar as bases de sistemas de "dupla acumulação", no qual todas as sentenças são "alcançadas" ou por meio da acumulação da verdade ou por meio da acumulação da falsidade. Tais sistemas parecem interessantes devido à possibilidade de introduzirmos axiomas nos quais os predicados de verdade e falsidade interagem.

Outro truque mais simples e possivelmente menos flexível é baseado nas cláusulas de acumulação definidas logo abaixo.

Definição K. As sentenças $\forall x\left(\left(\operatorname{SENT}_{n}(x) \rightarrow\left(\mathrm{V}_{n}(x) \leftrightarrow \mathrm{V}(x)\right)\right)\right.$, onde $\operatorname{SENT}_{n}(x)$ é uma contrapartida formal de " $x$ é uma sentença de $L_{P . A}$ de complexidade $n$ ", serão chamadas cláusulas de acumulação.

De fato, uma definição satisfatória desses sistemas de acumulação simples e dupla, bem como o estudo um pouco mais detalhado dos primeiros serão o objetivo desse trabalho. 


\section{Conceitos metateóricos fundamentais}

\section{$\S 1$. Linguagens de base}

Chamaremos as linguagens $L_{\text {P.A. }} L_{\text {P.A.V. }} L_{\text {P.A.F. }}$ e $L_{\text {P.A.V.F., definidas abaixo, }}$ coletivamente, linguagens de base.

As expressões das linguagens de base serão todas formuladas no alfabeto:

$$
\forall, \quad \exists, \quad \sim, \quad \vee, \wedge, \quad r, \quad f, \quad v, \quad \text { '. }
$$

Expressaremos os graus (ou as "aridades") dos símbolos funcionais e dos símbolos de predicado, respectivamente, pela repetição de ' $f$ ' e ' $r$ '; repetições de '”, por sua vez, indicarão o lugar dos símbolos na possível enumeração dos mesmos.

Os símbolos $f^{\prime}, f^{\prime \prime}, f^{\prime \prime \prime}, \ldots$ serão símbolos funcionais de grau 0 ou constantes individuais e serão abreviados, respectivamente, por $c_{1}, c_{2}, c_{3}, \ldots ; f f^{\prime}, f f^{\prime \prime}, f f^{\prime \prime \prime}, \ldots$ serão símbolos funcionais de grau 1 e serão abreviados por $f_{1}^{1}, f_{2}^{1}, f_{3}^{1}, \ldots$; enquanto que $f f f^{\prime}, f f f^{\prime \prime}, f f f^{\prime \prime \prime}, \ldots$ serão símbolos funcionais de grau 2 e serão abreviados por $f_{1}^{2}, f_{2}^{2}, f_{3}^{2}, \ldots$; e assim sucessivamente.

Os símbolos $r^{\prime}, r^{\prime \prime}, r^{\prime \prime \prime}, \ldots$ serão, por sua vez, símbolos de predicados (de grau 1) e serão abreviados por $P_{1}, P_{2}, P_{3}, \ldots ; r r^{\prime}, r r^{\prime \prime}, r r^{\prime \prime \prime}, \ldots$ serão símbolos de relações binárias e serão abreviados por $R_{1}^{2}, R_{2}^{2}, R_{3}^{2}, \ldots$; enquanto que $r r r^{\prime}, r r r^{\prime \prime}, r r r^{\prime \prime \prime}, \ldots$ serão símbolos de relações ternárias e serão abreviados por $R_{1}^{3}, R_{2}^{3}, R_{3}^{3}, \ldots$; e assim sucessivamente.

Os símbolos $c_{1}$ e $f_{1}^{1}$ serão tomados, respectivamente, como nomes de 0 e da função sucessor. De modo que:

1.1. Definição de numeral. As expressões $c_{1}, f_{1}^{1} c_{1}, f_{1}^{1} f_{1}^{1} c_{1}, f_{1}^{1} f_{1}^{1} f_{1}^{1} c_{1}, \ldots$ serão os numerais (formais) das linguagens de base. 
Abreviaremos os numerais formais, como se tornou usual, por meio de uma barra transversal sobre os numerais da aritmética informal. Desse modo, temos $\overline{0}, \overline{1}, \overline{2}, \overline{3}, \ldots$, tomados em sequência, como abreviações de numerais (formais).

Os símbolos $f_{1}^{2}, f_{2}^{2}$ e $f_{3}^{2}$ serão tomados, respectivamente, como nomes das operações binárias de adição, multiplicação e exponenciação.

1.2. Definição de termo fechado. Uma expressão $\tau$ é um termo fechado das linguagens de base:

a) Se $\tau$ é um numeral formal.

b) Ou se $\tau$ é da forma $f_{1}^{2} v v, f_{2}^{2} v v$ e $f_{3}^{2} v v$, onde $v$ e $v$ são termos fechados.

Escreveremos '+' no lugar de $f_{1}^{2} \mathrm{e}$ '?' no lugar de $f_{2}^{2}$ e utilizaremos, além disso, os símbolos de soma e multiplicação como infixos ${ }^{(1)}$. Desse modo, temos, primeiro, $+v v$ no lugar de $f_{1}^{2} v v$ e $\cdot v v$ no lugar de $f_{2}^{2} v v$; e, finalmente, $(v+v)$ no lugar de $+v v$ e $(v \cdot v)$ no lugar de $\cdot v v$. Escreveremos, ainda, $v v$ no lugar de $f_{3}^{2} v v$.

1.3. Definição de variável. As expressões $v^{\prime}, v^{\prime \prime}, v^{\prime \prime \prime}, \ldots$ serão as variáveis das linguagens de base.

Abreviaremos as variáveis por meio de subscritos; desse modo, temos $v_{1}, v_{2}$, $v_{3}, \ldots$ como abreviações das variáveis das linguagens de base. Escreveremos, além disso, $x$ no lugar de $v_{1} ; y$ no lugar de $v_{2} ; z$ no lugar de $v_{3} ; w$ no lugar de $v_{4} ; v$ no lugar de $v_{5} ; u$ no lugar de $v_{6} ; x_{1}$ no lugar de $v_{7} ; y_{1}$ no lugar de $v_{8} ;$ e assim sucessivamente.

1.4. Definição de termo. Uma expressão $\tau$ é um termo das linguagens de base:

a) Se $\tau$ é numeral formal ou uma variável.

b) Ou se $\tau$ é da forma $f_{1}^{2} v v, f_{2}^{2} v v$ e $f_{3}^{2} v v$, onde $v$ e $v$ são termos.

1. Para uma discussão sobre os tipos de afixos utilizados em lógica cf. Curry [1977, pp. 34-35]. 
As convenções notacionais para termos fechados continuarão, mutatis mutandis, valendo para termos tanto abertos quanto fechados.

Os símbolos $R_{1}^{2}$ e $R_{2}^{2}$ serão tomados, respectivamente, como nomes das relações numéricas de identidade e ordem. De modo que:

\subsection{Definição de fórmula atômica de $L_{P . A}$ :}

a) Se $\tau$ e $v$ são termos, então $R_{1}^{2} \tau v$ e $R_{2}^{2} \tau v$ são fórmulas atômicas de $L_{\text {P.A.: }}$.

Escreveremos '=' no lugar de $R_{1}^{2}$ e ' $\leqslant$ ' no lugar de $R_{2}^{2}$ e utilizaremos, além disso, os símbolos de identidade e ordem como infixos. Desse modo, teremos $\tau=v$ no lugar de $R_{1}^{2} \tau v$ e $\tau \leqslant v$ no lugar de $R_{2}^{2} \tau v$.

\subsection{Definição de fórmula atômica de $L_{\text {P.A.V., }} L_{\text {P.A.F. }}$ e $L_{\text {P.A.V.F. }}$}

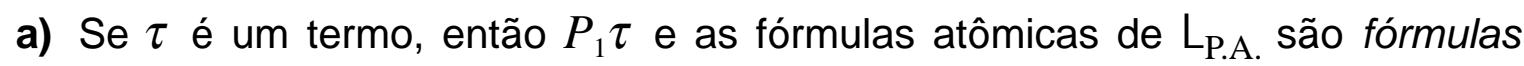
atômicas de $L_{\text {P.A.V. }}$ e $L_{\text {P.A.V.F. }}$

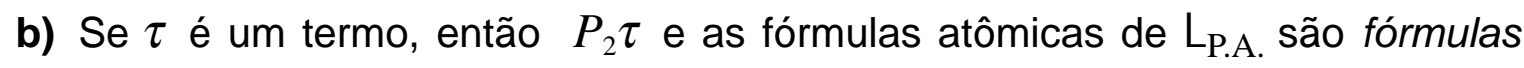
atômicas de $L_{\text {P.A.F. }}$ e $L_{\text {P.A.V.F. }}$

Escreveremos ' $\mathrm{V}$ ' no lugar de $P_{1}$ e 'F' no lugar de $P_{2}$ e adicionaremos ocasionalmente parênteses aos termos correspondentes. Desse modo, temos $\mathrm{V} \tau$ no lugar de $P_{1} \tau$ ou, às vezes, $\mathrm{V}(\tau)$ no lugar de $P_{1} \tau$; e $\mathrm{F} \tau$ no lugar de $P_{2} \tau$ ou, às vezes, $\mathrm{F}(\tau)$ no lugar de $P_{2} \tau^{(2)}$.

\subsection{Definição de fórmula das linguagens de base.}

a) As fórmulas atômicas de $L_{P . A}$ são fórmulas de $L_{P . A}$.

b) Se $\alpha$ e $\beta$ são fórmulas de $L_{\text {P.A. }}$ e $v$ é uma variável, então $\sim \alpha, v \alpha \beta, \wedge \alpha \beta, \forall v \alpha$ e $\exists v \alpha$ são fórmulas de $L_{P . A}$.

2. A ambiguidade não é de modo algum problemática, uma vez que os parênteses não ocorrem oficialmente em nenhuma das fórmulas desse estudo. 
Cláusulas análogas devem ser aplicadas às fórmulas atômicas de L.A.V. $L_{\text {P.A.F. }}$ e L.A.V.F. na definição das fórmulas das respectivas linguagens.

Escreveremos os conectivos ' $\vee$ ' e ' $\wedge$ ' como infixos (utilizando, é claro, adequadamente os parênteses); ' $\rightarrow$ ' e ‘ $\leftrightarrow$ ', por sua vez, serão introduzidos por meio das abreviações usuais: e. g., $(\alpha \rightarrow \beta)$ será a abreviação de $(\sim \alpha \vee \beta)$. Além disso, os parênteses serão associados à direita; os conectivos ' $\vee$ ' e ' $\wedge$ ' dominarão ' $\rightarrow$ ' e ' $\leftrightarrow$ '; ' ' dominará as demais constantes.

\subsection{Definição de substituição de variáveis livres por termos fechados. Seja} $v$ uma variável e $\tau$ um termo fechado:

a) Se $\alpha$ é uma fórmula atômica, $\alpha^{V} / \tau$ é o resultado da substituição de $v$ por $\tau$ em todas ocorrências de $v$ em $\alpha$;

b) $(\sim \alpha)^{v / \tau}=\sim(\alpha v / \tau)$;

c) $(\alpha \vee \beta)^{v / \tau}=\left(\alpha^{v / \tau}\right) \vee\left(\beta^{v / \tau}\right)$;

d) $(\alpha \wedge \beta)^{v / \tau}=\left(\alpha^{v / \tau}\right) \wedge\left(\beta^{v / \tau}\right)$

e) $(\forall v \alpha)^{v / \tau}=\forall v \alpha$;

f) $(\exists v \alpha)^{v / \tau}=\exists v \alpha$;

Se $\xi$ é uma variável diferente de $v$, então:

g) $(\forall v \alpha)^{\xi / \tau}=\forall v(\alpha \xi / \tau)$.

h) $(\exists v \alpha)^{\xi} / \tau=\exists v(\alpha \xi / \tau){ }^{(3)}$

Os conceitos de variável livre e ligada, de sentença e de complexidade ou grau de uma fórmula serão os usuais [cf. Smullyan, 1992, pp. 15-16].

A possível adição de símbolos funcionais ou relacionais às linguagens de base deve ser acompanhada de modificações óbvias nas cláusulas relevantes das definições acima.

3. Uma definição de substituição desse tipo pode ser encontrada em First-Order Logic de Smullyan [1995, p. 44]. 


\section{§2. Alguns conceitos semânticos}

2.1. Definição de denotação. A denotação $d(\tau)$ do termo fechado $\tau$ é definida pelas cláusulas abaixo:

a) Para cada numeral $\bar{n}, d(\bar{n})=n$.

b) Se $\tau$ e $v$ são termos fechados, então $d\left(f_{1}^{2} \tau v\right)$ é a soma de $d(\tau)$ e $d(v)$; $d\left(f_{2}^{2} \tau v\right)$ é a multiplicação de $d(\tau)$ por $d(v) ; d\left(f_{3}^{2} \tau v\right)$ é a $d(v)$-ésima potência $\operatorname{de} d(\tau)$.

Assim teremos, por exemplo, que $d\left(f_{2}^{2} f_{1}^{2} f_{1}^{1} f_{1}^{1} c_{1} f_{1}^{1} f_{1}^{1} c_{1} f_{1}^{1} f_{1}^{1} c_{1}\right)=8$, ou seja, que $d((\overline{2}+\overline{2}) \cdot \overline{2})=8$.

As cláusulas de b) são enunciadas "por extenso" para dissipar qualquer aparência de trivialidade ou ambiguidade sugerida pelas abreviações: $d(\tau+v)=d(\tau)+d(v)$; $d(\tau \cdot v)=d(\tau) \cdot d(v) ; d\left(\tau^{v}\right)=d(\tau) d(v)$.

\subsection{Definição de verdade em $\mathbb{N}$ para sentenças atômicas de $L_{P . A}$ :}

Se $\alpha$ é uma sentença atômica de $L_{P . A}, \alpha$ é da forma $R_{1}^{2} \tau \nu$ ou $\alpha$ é da forma $R_{2}^{2} \tau \nu$. Desse modo:

a) No primeiro caso, $\alpha$ é verdadeira em $\mathbb{N}$ se e somente se $d(\tau)$, a denotação de $\tau$, e $d(v)$, a denotação de $v$, são iguais.

b) No segundo caso, $\alpha$ é verdadeira em $\mathbb{N}$ se e somente se $d(\tau)$ é menor que $d(v)$ ou $d(\tau)$ e $d(v)$ são iguais.

Na versão abreviada, teríamos: a) se $\alpha$ é $\tau=v$, então $\alpha$ é verdadeira em $\mathbb{N}$ sse $d(\tau)=d(v) ;$ b) Se $\alpha$ é $\tau \leqslant v$, então $\alpha$ é verdadeira sse $d(\tau) \leqslant d(v)$.

\subsection{Definição de verdade em $\mathbb{N}$ para sentenças de $L_{\text {P.A. }}$ (complemento).}

Para qualquer sentença não-atômica $\varphi$ de $L_{\text {P.A. }}$, temos:

a) Se $\varphi$ é da forma $\sim \alpha$, $\varphi$ é verdadeira em $\mathbb{N}$ se somente se $\alpha$ não é verdadeira $e m \mathbb{N}$. 
b) Se $\varphi$ é $(\alpha \vee \beta)$, $\varphi$ é verdadeira em $\mathbb{N}$ sse $\alpha$ é verdadeira em $\mathbb{N}$ ou $\beta$ é verdadeira em $\mathbb{N}$.

c) Se $\varphi$ é da forma $(\alpha \wedge \beta)$, $\varphi$ é verdadeira $(e m \mathbb{N})$ sse $\alpha$ é verdadeira em $\mathbb{N}$ e $\beta$ é verdadeira em $\mathbb{N}$.

d) Se $\varphi$ é $\forall v \alpha$, $\varphi$ é verdadeira sse $\alpha^{v} / \bar{n}$ é verdadeira em $\mathbb{N}$ para todo número natural $n$.

e) E, finalmente, se $\varphi$ é da forma $\exists v \alpha$, $\varphi$ é verdadeira sse $\alpha^{V /} / \bar{n}$ é verdadeira em $\mathbb{N}$ para algum natural $n$.

2.4. Notação alternativa para verdade em $\mathbb{N}$. Escreveremos, também, $\mathbb{N} \vDash \alpha$ no lugar de $\alpha$ é verdadeira $(e m \mathbb{N})$.

\subsection{Definição dos conjuntos de verdades e falsidades de $L_{P . A}$ :}

a) $\hat{\mathrm{V}}=\{\alpha \mid \alpha$ é verdadeira $\}=\{\alpha \mid \mathbb{N} \vDash \alpha\}$.

b) $\hat{\mathrm{F}}=\{\alpha \mid \sim \alpha$ é verdadeira $\}$.

\subsection{Alguns fatos simples sobre $\hat{V}$ e $\hat{F}$.}

a) $\hat{\mathrm{F}} \cap \hat{\mathrm{V}}=\varnothing$.

b) $\hat{F} \cup \hat{V}=$ Conjunto das sentenças de $L_{P . A}$ :

c) As condições booleanas valem para $\hat{V}$ e $\hat{F}$, e. g., se $\alpha \in \hat{V}$, então $(\alpha \vee \beta) \in \hat{V}$; se $\alpha \in \hat{\mathrm{F}}$, então $(\alpha \wedge \beta) \in \hat{\mathrm{F}}$.

d) Se $\forall v \alpha \in \hat{\mathrm{V}}$, então, para todo termo fechado $\tau, \alpha v / \tau \in \hat{\mathrm{V}}$.

e) Se $\exists v \alpha \in \hat{V}$, então, para algum termo fechado $\tau, \alpha v / \tau \in \hat{V}$.

Seja $\varphi\left(v_{1}, v_{2}, \ldots, v_{n}\right)$ uma fórmula cujas variáveis livres são exatamente $v_{1}, v_{2}, \ldots$, $v_{n}$; entenderemos $\varphi\left(\tau_{1}, \tau_{2}, \ldots, \tau_{n}\right)$ como o resultado da substituição sucessiva das variáveis $v_{1}, v_{2}, \ldots, v_{n}$ pelos termos fechados $\tau_{1}, \tau_{2}, \ldots, \tau_{n}$ em $\varphi$, em outras palavras, $\varphi\left(\tau_{1}, \tau_{2}, \ldots, \tau_{n}\right)$ será $\left(\ldots\left(\left(\varphi^{v_{1}} / \tau_{1}\right) v_{2} / \tau_{2}\right) \ldots v_{n} / \tau_{n}\right)$. 
2.7. Definição de propriedade ou relação expressável em $L_{P . A}$. Uma relação $R n$-ária é expressávelem $L_{\text {P.A. }}$ se existe uma fórmula $\varphi$ de $L_{\text {P.A. }}$ tal que, para quaisquer números naturais $k_{1}, k_{2}, \ldots, k_{n}, \varphi\left(\bar{k}_{1}, \bar{k}_{2}, \ldots, \bar{k}_{n}\right)$ é verdadeira sse $\left\langle k_{1}, k_{2}, \ldots, k_{n}\right\rangle \in R$.

\subsection{Alguns exemplos de relações expressáveis em $L_{P . A}$ :}

a) " $x$ é o antecessor de $y$ " é expressa por $f_{1}^{1} x=y$.

b) " $x$ é par" é expressa por $\exists y(y+y=x)$ e, também, por $\exists y(\overline{2} \cdot y=x)$.

c) " $x$ é divisível por $y$ " é expressa por $\exists z(y \cdot z=x)$.

2.9. Definição da notação de barra simples. Por $|\varphi|$, entenderemos a relação expressa por $\varphi$ em $L_{\text {P.A. }}$ (Não devemos confundir $|\exists y(y+y=x)|$ que se refere ao conjunto dos pares e $\lceil\exists y(y+y=x)\rceil$ que se refere ao numeral de Gödel da fórmula [cf. p. 53].)

\subsection{Alguns fatos triviais sobre a notação de barra simples.}

a) Embora $\exists y(y+y=x), \exists y(\overline{2} \cdot y=x)$ e $\exists y(y \leqslant x \wedge y+y=x)$ sejam todas fórmulas diferentes de $L_{\text {P.A. }}|\exists y(y+y=x)|=|\exists y(\overline{2} \cdot y=x)|=|\exists y(y \leqslant x \wedge y+y=x)|$.

b) As condições booleanas valem para $\left|\varphi\left(v_{1}, \ldots, v_{n}\right)\right| \mathrm{e}\left|\psi\left(v_{1}, \ldots, v_{n}\right)\right|$, por exemplo, $\left|\varphi\left(v_{1}, \ldots, v_{n}\right)\right| \cup\left|\psi\left(v_{1}, \ldots, v_{n}\right)\right|=\left|\varphi\left(v_{1}, \ldots, v_{n}\right) \vee \psi\left(v_{1}, \ldots, v_{n}\right)\right| ;\left|\varphi\left(v_{1}, \ldots, v_{n}\right)\right| \cap \mid \psi\left(v_{1}\right.$, $\left.\ldots, v_{n}\right)|=| \varphi\left(v_{1}, \ldots, v_{n}\right) \wedge \psi\left(v_{1}, \ldots, v_{n}\right) \mid$.

Obviamente, podemos expressar em $L_{\text {P.A. }}$ relações para as quais nenhum nome foi cunhado, diferentemente dos usuais " $x$ é par" e " $x$ é divisível por $y$ "; nesses casos, a notação de barra simples pode ser bastante útil.

Temos, então, que:

2.11. Definição da relação de complexidade na Base 10. Seja $\mathrm{CX}(x) \approx y$ uma abreviação de $\forall x_{1}\left(x_{1} \leqslant y \rightarrow\left(f_{1}^{1} x_{1}=y \rightarrow \overline{10}^{x_{1}} \leqslant x\right)\right) \wedge f_{1}^{1} x \leqslant \overline{10}^{y}$; entenderemos a relação " $y$ é a complexidade de $x$ (na base 10)" como $|\mathrm{CX}(x) \approx y|$.

Assim, por exemplo, "2 é a complexidade de 11" ou $\langle 11,2\rangle \in|\mathrm{CX}(x) \approx y|$, porque $\mathrm{CX}(\overline{11}) \approx \overline{2}$ é verdadeira $(\mathrm{em} \mathbb{N})$. 
O símbolo (informal) ‘ $\approx$ ’ será sempre parte de um símbolo, por assim dizer, mais complexo, de modo que ' $\approx$ ' não terá um significado independente propriamente dito. Não obstante, o símbolo indicará que estamos trabalhando com relações de um tipo especial, mais especificamente, com gráficos de funções; assim, ' $\approx$ ' será uma espécie de símbolo de (pseudo-)identidade que será usado com propósitos mnemônicos.

Podemos, é claro, expressar funções por meio de termos da linguagem, e. g., "a $x$-ésima potência de $x$ " é expressa por $x^{x}$. Entretanto, a capacidade expressiva dos termos das linguagens de base é bastante limitada, é por isso que o símbolo ' $\approx$ ' é tão útil; não temos, por exemplo, termos correspondentes às relações $|\mathrm{CX}(x) \approx y| \mathrm{e}$ $\left|\left(f_{1}^{1} x=y \vee y=\overline{0}\right)\right|$ (" $x$ é o antecessor de $y$ ").

2.12. Mais sobre gráficos. Se $R$ é o gráfico de uma função $n$-ária $f$, entenderemos $R\left(k_{1}, \ldots, k_{n}\right)$, onde $k_{1}, \ldots, k_{n}$ são números naturais, como $f\left(k_{1}, \ldots, k_{n}\right)$, ou seja, o valor da função para os argumentos dados; além disso, entenderemos $R\left(v_{1}, \ldots, v_{n}\right)$, onde $v_{1}, \ldots, v_{n}$ são variáveis, como a própria função $f$.

Como um caso especial temos: seja $|\varphi|$ o gráfico da função $n$-ária fe $k_{1}, \ldots, k_{n}$ números naturais; por $|\varphi|\left(k_{1}, \ldots, k_{n}\right)$, entenderemos o valor de f para os argumentos $k_{1}, \ldots, k_{n}$, em outras palavras, $|\varphi|\left(k_{1}, \ldots, k_{n}\right)=\mathrm{f}\left(k_{1}, \ldots, k_{n}\right)$.

\section{§3. Introdução à hierarquia da aritmética}

A hierarquia das fórmulas de $L_{P . A}$ será definida de modo ligeiramente diferente dos mais usuais (pelo menos, fora da comunidade dos cientistas da computação).

Devemos notar, sobretudo, dois aspectos interrelacionados: primeiro, $f_{3}^{2}$ (um símbolo para "a ... -ésima potência de ..." pela interpretação padrão [cf. 2.1, p. 44]) é parte do vocabulário primitivo das linguagens de base [cf. 1.2, p. 41]; segundo, permitiremos que as fórmulas- $\Sigma_{0}$, que formam a base de nossa hierarquia, sejam limitadas 
por termos quaisquer e não apenas por variáveis (ou, às vezes, numerais e outros termos fechados). Essas modificações nos possibilitarão estabelecer contrapartidas formais $\Sigma_{0}$ de alguns conceitos metateóricos que, de outro modo, requereriam contrapartidas tanto $\Sigma_{1}$ quanto $\Pi_{1}$.

\subsection{Definição de fórmulas- $\Sigma_{0}$.}

a) Todas fórmulas atômicas de $L_{\text {P.A. }}$ são fórmulas $-\Sigma_{0}$.

b) Se $\alpha$ e $\beta$ são fórmulas- $\Sigma_{0}$, então $\sim \alpha,(\alpha \vee \beta)$ e $(\alpha \wedge \beta)$ são fórmulas- $\Sigma_{0}$ (e, portanto, também $(\alpha \rightarrow \beta)$ e $(\alpha \leftrightarrow \beta))$.

c) Se $\alpha$ é uma fórmula- $\Sigma_{0}, v$ é uma variável e $\tau$ é (1) um termo fechado ou (2) um termo cujas variáveis são distintas de $v$, então $\exists v(v \leqslant \tau \wedge \alpha)$ e $\forall v(v \leqslant \tau \rightarrow \alpha)$ são fórmulas $-\Sigma_{0} \cdot{ }^{(4)}$

\subsection{Fatos simples sobre fórmulas $-\Sigma_{0}$.}

a) Se $\varphi$ é uma sentença- $\Sigma_{0}$, então a verdade ou falsidade de $\varphi$ em $\mathbb{N}$ é decidível.

b) Se $\varphi$ é uma fórmula- $\Sigma_{0}$ (aberta), então $|\varphi|$ é uma relação decidível.

\subsection{Convenções notacionais.}

a) Escreveremos, como é usual, $\exists v \leqslant \tau(\alpha)$ no lugar de $\exists v(v \leqslant \tau \wedge \alpha)$ e $\exists v<\tau(\alpha)$ no lugar de $\exists v\left(f_{1}^{1} v \leqslant \tau \wedge \alpha\right)$;

b) Escreveremos, também, $\forall v \leqslant \tau(\alpha)$ no lugar de $\forall v(v \leqslant \tau \rightarrow \alpha)$ e $\forall v<\tau(\alpha)$ no lugar de $\forall v\left(f_{1}^{1} v \leqslant \tau \rightarrow \alpha\right)$.

\subsection{Definição da hierarquia da aritmética.}

a) Se $\alpha$ é uma fórmula- $\Sigma_{0}$ e $v$ é uma variável; então (1) $\alpha$ e $\exists v(\alpha)$ são fórmulas $-\Sigma_{1}$ e (2) $\alpha$ e $\forall v(\alpha)$ são fórmulas- $\Pi_{1}$.

4. O que difere tanto de Hájek \& Pudlák [1998, p. 13] quanto de Smullyan [1992, p. 41], as principais fontes dos conceitos mais fundamentais desse estudo; Hájek e Pudlák permitem apenas variáveis como "limitadores", enquanto Smullyan permite tanto variáveis quanto numerais. No caso particular da hierarquia, estamos mais próximos, por exemplo, de Buss [1998, pp. 82-83] e Franzén [2004, pp.136-138]. 
b) Se $\alpha$ é uma fórmula- $\Sigma_{n}$, onde $1 \leqslant n$, e $v$ é uma variável; então $\alpha$ e $\forall v(\alpha)$ são fórmulas- $\prod_{n+1}$.

c) Se $\alpha$ é uma fórmula- $\prod_{n}$, onde $1 \leqslant n$, e $v$ é uma variável; então $\alpha$ e $\exists v(\alpha)$ são fórmulas- $\sum_{n+1}$.

3.5. Definição da hierarquia de relações. Uma relação $R$ será $\Sigma_{n}$ se for expressável por uma fórmula- $\Sigma_{n} ; R$ será $\Pi_{n}$ se for expressável por uma fórmula- $\Pi_{n} ; R$ será $\Delta_{n}$ se for expressável tanto por uma fórmula- $\Sigma_{n}$ quanto por uma fórmula- $\prod_{n}$ (ou se tanto $R$ quanto seu complemento forem expressáveis por fórmulas- $\Sigma_{n}$ ou, ainda, se tanto $R$ quanto seu complemento forem expressáveis por fórmulas- $\left.\prod_{n}\right) .{ }^{(5)}$

\subsection{Alguns exemplos e fatos simples.}

a) " $x$ é par" é $\Sigma_{0}$ (e $\Sigma_{1}, \Pi_{1}$, etc.), uma vez que $\{x \mid x$ é par $\}=|\exists y \leqslant x(y+y=x)|$ (ou que $\{x \mid x$ é par $\}=|\exists y \leqslant x(\overline{2} \cdot y=x)|$ );

b) $|\mathrm{CX}(x) \approx y| \mathrm{e}\left|\exists w \leqslant \overline{2}^{x}(x \cdot w=y)\right|$ são $\Sigma_{0}{ }^{(6)}$;

c) Se $R$ é o gráfico de uma função e $R$ é $\Sigma_{n}$, então $R$ é, também, uma relação $\prod_{n}$. Se $R$ é o gráfico de uma função e $R$ é $\Pi_{n}$, então $R$ é, também, $\Sigma_{n}$.

\section{\$4. Sistemas formais}

Uma vez que nosso estudo estará centrado na formalização de conceitos semânticos (e, incidentalmente, na sintaxe dos termos e fórmulas), as noções metateóricas ligadas ao conceito de derivação serão apenas esboçadas.

5. O seguinte resumo de resultados será útil àqueles que estão familiarizados com os conceitos básicos da teoria da recursão: as relações $\Sigma_{0}=\Pi_{0}=\Delta_{0} \subset$ as relações recursivas primitivas $\subset \Delta_{1}=$ as relações recursivas $\subset \Sigma_{1}=$ as relações recursivamente enumeráveis [cf., por exemplo, Kaye, 1991, pp. 28-34].

6. Devemos notar que não teríamos subsídios para afirmar que essa segunda relação é ou não é $\Sigma_{0}$ se entendêssemos a hierarquia da aritmética como Smullyan ou Hájek \& Pudlák a entendem [cf. nota anterior, p. 28]. Contudo, os únicos fatos importantes para nós são: que as relações $\Sigma_{0}$ são decidíveis [cf. 3.2, p. 48] e que as instâncias relevantes das contrapartidas formais dessas relações são deriváveis nos sistemas formais discutidos nesse estudo. 
4.1. Definição. Aos axiomas usuais do sistema Q de Robinson [cf., por exemplo, Smullyan, 1992, p. 29], acrescentaremos:

a) $\forall x\left(f_{3}^{2} x \overline{0}=\overline{1}\right)$;

b) $\forall x \forall y\left(f_{3}^{2} x f_{1}^{1} y=f_{3}^{2} x y \cdot x\right)$.

Ou, mais familiarmente, usando nossas convenções notacionais [cf. pp. 41-42]:

a) $\forall x\left(x^{\overline{0}}=\overline{1}\right)$;

b) $\forall x \forall y\left(x^{f_{1}^{1} y}=x^{y} \cdot x\right)$.

Ou seja, acrescentaremos os axiomas recursivos de "a ...-ésima potência de _...".

O mais importante de nossos sistemas formais será denotado por P.A. (apesar da introdução, não de todo usual, da exponenciação).

4.2. Definição de P.A.. Os axiomas de P.A. serão:

a) Os axiomas do sistema Q de Robinson:

$\left.\mathrm{Q}_{1}\right) \quad \forall x \forall y\left(f_{1}^{1} x=f_{1}^{1} y \rightarrow x=y\right)$;

$\left.\mathrm{Q}_{2}\right) \quad \forall x\left(\sim f_{1}^{1} x=\overline{0}\right)$;

$\left.\mathrm{Q}_{3}\right) \quad \forall x\left(\sim x=\overline{0} \rightarrow \exists y\left(x=f_{1}^{1} y\right)\right)$;

$\left.\mathrm{Q}_{4}\right) \quad \forall x(x+\overline{0}=x)$;

$\left.\mathrm{Q}_{5}\right) \quad \forall x \forall y\left(x+f_{1}^{1} y=f_{1}^{1}(x+y)\right)$;

Q $) \quad \forall x(x \cdot \overline{0}=\overline{0})$;

$\left.\mathrm{Q}_{7}\right) \quad \forall x \forall y\left(x \cdot f_{1}^{1} y=(x \cdot y)+x\right)$;

$\left.\mathrm{Q}_{8}\right) \quad \forall x(x \leqslant \overline{0} \leftrightarrow x=\overline{0})$;

$\left.\mathrm{Q}_{9}\right) \quad \forall x \forall y\left(x \leqslant f_{1}^{1} y \leftrightarrow x \leqslant y \vee x=f_{1}^{1} y\right)$;

$\left.\mathrm{Q}_{10}\right) \forall x \forall y(x \leqslant y \vee y \leqslant x)$.

b) Os axiomas 4.1. a) e b);

c) As induções $\varphi(\overline{0}) \wedge \forall x\left(\varphi(x) \rightarrow \varphi\left(f_{1}^{1} x\right)\right) \rightarrow \forall x(\varphi(x))$, onde $\varphi$ é qualquer fórmula de $L_{P . A}$. 
Um fragmento útil de P.A. será $\mathrm{I} \Sigma_{1}$.

4.3. Definição de $\mathrm{I} \Sigma_{1}$. Os axiomas de $\mathrm{I} \Sigma_{1}$ serão:

a) Os axiomas do sistema $Q$ de Robinson;

b) Os axiomas 4.1. a) e b);

c) As induções $\varphi(\overline{0}) \wedge \forall x\left(\varphi(x) \rightarrow \varphi\left(f_{1}^{1} x\right)\right) \rightarrow \forall x(\varphi(x))$, onde $\varphi$ deverá ser uma fórmula $\Sigma_{1}$ de $L_{\text {P.A. }}$.

4.4. Definição de $Q^{*}$. Os axiomas do sistema $Q$ de Robinson juntamente com os axiomas 4.1. a) e b) serão referidos como axiomas $Q^{*}$.

Suponhamos, então, um cálculo lógico (no estilo hilbertiano) bem constituído, de modo que os conceitos de derivação a partir de P.A., I $\Sigma_{1}$ e $Q^{*}$, bem como de teorema de P.A., I $\Sigma_{1}$ e $\mathrm{Q}^{*}$ estejam adequadamente definidos. Escreveremos $\mathrm{Q}^{*} \vdash \alpha$, se $\alpha$ é um teorema de $\mathrm{Q}^{*}$, P.A. $\vdash \alpha$, se $\alpha$ é um teorema de P.A. e I $\Sigma_{1} \vdash \alpha$, se $\alpha$ é um teorema de $\mathrm{I} \Sigma_{1}$

É óbvio, então, que todos os teoremas de $\mathrm{Q}^{*}$ e I $\Sigma_{1}$ são teoremas de P.A.e que todos os teoremas de $\mathrm{Q}^{*}$ são teoremas de $\mathrm{I} \Sigma_{1}$.

Analogamente ao conceito de expressável [cf. p. 46] (em relação ao modelo padrão), temos o conceito de representável em um sistema formal dado:

4.5. Definição de representação. Uma relação $R n$-ária é representável em $\mathrm{S}$ se existe uma fórmula $\varphi$ de $\mathrm{L}_{\text {P.A. }}$ tal que, para quaisquer números naturais $k_{1}, \ldots, k_{n},\left\langle k_{1}, \ldots\right.$, $\left.k_{n}\right\rangle \in R$ sse $\mathrm{S} \vdash \varphi\left(\bar{k}_{1}, \ldots, \bar{k}_{n}\right) ;$ no caso, $\varphi$ é uma representação de $R$ em $\mathrm{S}$.

4.6. Definição de bi-representação. Uma relação $R$ n-ária é bi-representável em $\mathrm{S}$ se existe uma fórmula $\varphi$ de $\mathrm{L}_{\text {P.A. }}$ tal que, para quaisquer números naturais $k_{1}, \ldots, k_{n},\left\langle k_{1}, \ldots, k_{n}\right\rangle \in R$ sse $\mathrm{S} \vdash \varphi\left(\bar{k}_{1}, \ldots, \bar{k}_{n}\right)$ e $\left\langle k_{1}, \ldots, k_{n}\right\rangle \notin R$ sse $\mathrm{S} \vdash \sim \varphi\left(\bar{k}_{1}, \ldots, \bar{k}_{n}\right)$, no caso, $\varphi$ é uma bi-representação de $R$ em $\mathrm{S}$. 
III

\section{Gödelização da metateoria}

\section{§1. Introdução à gödelização da sintaxe 1: expressões}

Apresentaremos nessa seção uma gödelização de nossas linguagens de base fundamentada na operação de concatenação. Para tanto, os números de Gödel das expressões serão definidos por meio da seguinte atribuição de números ao alfabeto das linguagens de base:

$\begin{aligned} \prime & \mapsto 0 & \vee & \mapsto 5 \\ f & \mapsto 1 & \wedge & \mapsto 6 \\ r & \mapsto 2 & \forall & \mapsto 7 \\ v & \mapsto 3 & \exists & \mapsto 8 \\ \sim & \mapsto 4 & & \end{aligned}$

Encontraremos os números de Gödel das expressões concatenando simplesmente os dígitos correspondentes aos elementos do alfabeto. Por exemplo, o número de Gödel de $\vee f \forall$ ou, mais sucintamente, $g(\vee f \forall)$ será 517 .

\subsection{Alguns exemplos de conjuntos de números de Gödel.}

a) $\{1110,11100,111000,110,10\}$ é o conjunto dos números de Gödel dos símbolos funcionais das linguagens de base.

b) $\{10,11010,11011010,11011011010, \ldots\}$ é o conjunto de números de Gödel dos numerais formais.

c) $\{20,220,2200\}$, ou seja, $\left\{g\left(r^{\prime}\right), g\left(r r^{\prime}\right), g\left(r r^{\prime \prime}\right)\right\}$ é o conjunto dos números de Gödel dos símbolos de predicado de LP.A.V.

d) A imagem de $30 \cdot 10^{x}$ (da função $30 \cdot 10^{x}$ ) ou, talvez melhor, a imagem de $\lambda x\left(30 \cdot 10^{x}\right)$ é o conjunto de números de Gödel das variáveis. 
1.2. Definição da notação de barra dupla. Seja $\varphi$ um predicado ou uma relação na metalinguagem das linguagens de base (ou seja, o domínio das variáveis livres de $\varphi$ deverá ser o conjunto das expressões das linguagens de base), $\left\langle k_{1}, k_{2}, \ldots, k_{n}\right\rangle \in\|\varphi\|$ se e somente se $\varphi\left(g^{-1}\left(k_{1}\right), g^{-1}\left(k_{2}\right), \ldots, g^{-1}\left(k_{n}\right)\right)$.

\subsection{Alguns exemplos.}

a) $\| x$ é um símbolo funcional de $L_{\text {P.A. }} \|=\{1110,11100,111000,110,10\}$;

b) $\| x$ é um predicado de $L_{\text {P.A.V. }} \|=|x=\overline{20} \vee x=\overline{220} \vee x=\overline{2200}|=\{20,220,2200\}$;

c) $\| x$ é uma variável $\|=\left|\exists z\left(30 \cdot 10^{z}=y\right)\right|=$ a imagem de $\lambda x\left(30 \cdot 10^{x}\right)$.

1.4. Definição de propriedade e relação g-expressável. Uma relação $R$ é gödelianamente expressável ou, mais sucintamente, $g$-expressável se existe uma fórmula $\varphi$ de $L_{\text {P.A. }}$ tal que, para quaisquer números naturais $k_{1}, k_{2}, \ldots, k_{n}, \varphi\left(\bar{k}_{1}, \bar{k}_{2}, \ldots, \bar{k}_{n}\right)$ é verdadeiro em $\mathbb{N}$ sse $\left\langle g^{-1}\left(k_{1}\right), g^{-1}\left(k_{2}\right), \ldots, g^{-1}\left(k_{n}\right)\right\rangle \in R$, ou seja, se $\|R\|=|\varphi|$.

1.5. Definição das aspas retas. $\lceil\varepsilon\rceil=\overline{g(\varepsilon)}$, onde $\varepsilon$ é uma expressão das linguagens de base; $g(\varepsilon)$ é um número natural e, portanto, $\lceil\varepsilon\rceil$ é um numeral; $\lceil\varepsilon\rceil$ é o numeral de Gödel da expressão $\varepsilon$.

\section{§2. Coletânea de contrapartidas formais relevantes}

As próximas seções desse capítulo serão dedicadas aos pormenores da descrição de algumas das contrapartidas formais necessárias à discussão posterior, notadamente, da descrição das contrapartidas formais relativas à tipologia das expressões das linguagens de base e à denotação. Antes disso, entretanto, apresentaremos algumas dessas fórmulas fora do contexto (no mais da vezes, bastante complexo) de suas definições com o objetivo de proporcionar alguma familiaridade com a notação que empregaremos nesse estudo. 


\subsection{Termos.}

a) $\operatorname{TM}(x)$ é uma contrapartida formal $\Sigma_{0}$ de "xé um termo das linguagens de base", ou seja, $\operatorname{TM}(x)$ é uma fórmula $\Sigma_{0}$ (e, portanto, de $L_{\text {P.A. }}$ ) tal que $\| x$ é um termo $\|=|\operatorname{TM}(x)|$ (e, portanto, TM $(x)$ é uma bi-representação do conjunto de números de Gödel dos termos das linguagens de base);

b) $\mathrm{TF}(x)$ é uma contrapartida formal $\Sigma_{0}$ de " $x$ é um termo fechado (das linguagens de base)";

c) $\operatorname{VAR}(x)$ é uma contrapartida formal $\Sigma_{0}$ de " $x$ é uma variável”;

d) $\mathrm{NU}(x)$ é uma contrapartida formal $\Sigma_{0}$ de " $x$ é um numeral".

\subsection{Fórmulas-legenda.}

a) $\operatorname{LTF}^{\Sigma}(x) \simeq y$ é uma contrapartida formal $\Sigma_{1}$ de " $y$ é a denotação do termo $x$ ";

b) $\operatorname{LTF}^{\Pi}(x) \simeq y$ é uma contrapartida formal $\Pi_{1}$ de " $y$ é a denotação do termo $x$ ".

\subsection{Fórmulas e sentenças.}

a) $\operatorname{FORM}(x)$ é uma contrapartida formal $\Sigma_{0}$ de " $x$ é uma fórmula de $L_{\text {P.A."; }}$

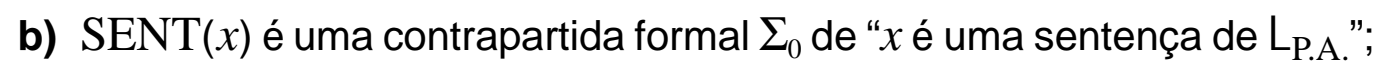

c) $\operatorname{SENT}_{n}(x)$ é uma contrapartida formal $\Sigma_{0}$ de " $x$ é uma sentença de $L_{\text {P.A. }}$ de complexidade $n "$.

É importante notar que $x$ é a única variável de $\operatorname{SENT}_{n}(x)$ e que $n$ é um parâmetro metalinguístico.

O que nós temos aqui é, de fato, uma sequência de certas fórmulas $\Sigma_{0}: \operatorname{SENT}_{0}(x)$, $\operatorname{SENT}_{1}(x), \ldots, \operatorname{SENT}_{n}(x), \ldots$, cada uma delas com uma única variável livre; embora, diferentemente do caso das DPVs [cf. p. 36], pudêssemos construir uma fórmula $\operatorname{SENT}_{y}(x)$ cujas variáveis livres são $x$ e $y$ tal que $\mathbb{N} \vDash \forall x\left(\exists y\left(\operatorname{SENT}_{y}(x)\right) \leftrightarrow \operatorname{SENT}(x)\right)$.

Exemplificaremos nosso uso da notação em diversas das fórmulas auxiliares empregadas nas discussões posteriores. Nossa esperança é que tais exemplos, 
somados às dicas encontradas no contexto da discussão relevante, sejam por si só capazes de permitir uma leitura adequada dos capítulos IV e V.

\subsection{Construção de expressões.}

a) $\doteq(x, y) \approx z$ é uma contrapartida formal $\Sigma_{0}$ de " $z$ é a equação cujos termos são $x$ e $y^{\prime \prime}$

b) $\dot{\sim}(x) \approx y$ é uma contrapartida formal $\Sigma_{0}$ de " $y$ é a negação de $x$ ".

Outras abreviações, nas quais símbolos pontuados e a igualdade ' $\approx$ ' ocorrem devem ser entendidas de maneira similar, e. $g ., \dot{\wedge}(x, y) \approx z$ é uma contrapartida formal $\Sigma_{0}$ de " $z$ é a conjunção de $x$ e $y$ ".

\subsection{Tipos de expressões.}

a) $\dot{\xi}(x)$ é uma contrapartida formal $\Sigma_{0}$ de " $x$ é uma inequação".

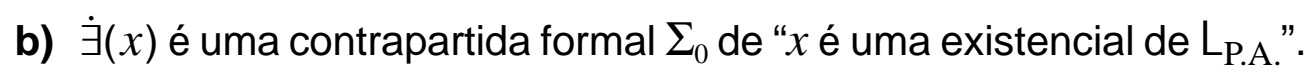

Como anteriormente, outras abreviações, nas quais apenas os símbolos pontuados aparecem, devem ser entendidas de maneira similar, e. $g ., \dot{\wedge}(x)$ é uma contrapartida formal $\Sigma_{0}$ de "x é uma conjunção de $L_{\text {P.A.". }}$

\subsection{Tipos de sentenças.}

a) $\operatorname{SENT} \doteq(x)$ é uma contrapartida formal $\Sigma_{0}$ de " $x$ é uma equação sem variáveis livres";

b) $\operatorname{SENT} \dot{v}(x)$ é uma contrapartida formal $\Sigma_{0}$ de " $x$ é uma disjunção sem nenhuma variável livre".

\subsection{Projeções.}

a) $\mathrm{P} \dot{\sim}(x) \simeq y$ é uma contrapartida formal $\Sigma_{0}$ de " $y$ é a fórmula cuja negação é $x$ ";

b) $\operatorname{PP} \dot{\vee}(x) \simeq y$ é uma contrapartida formal $\Sigma_{0}$ de " $y$ é o primeiro dos disjuntos de $x$ ";

c) $\operatorname{PS} \wedge(x) \simeq y$ é uma contrapartida formal $\Sigma_{0}$ de " $y$ é o segundo dos conjuntivos de $x "$. 


\section{§3. Codificação da teoria dos conjuntos e das sequências finitas}

Embora possamos tratar, no espírito da primeira seção, das contrapartidas aritmetizadas dos predicados e das relações metateóricos por meio de ferramentas informais conhecidas, como, por exemplo, a teoria da recursão; isso é insuficiente para nossos propósitos. Devemos, sistematicamente, mostrar que tais relações metateóricas são g-expressáveis (na verdade, que são g-expressáveis por um tipo especial de fórmula da hierarquia da aritmética). Para tanto, torna-se necessário simular uma teoria dos conjuntos finitos de números naturais por meio de predicados e relações $g$-expressáveis, de modo a estabelecer uma fundamentação da teoria da recursão na aritmética formal. Utilizaremos no que se segue uma codificação extensional dos conjuntos finitos inspirada em Ackermann [1937] e proposta por Hájek \& Pudlák [para maiores detalhes cf. 1998, pp. 37-44]; entretanto, evitaremos o desvio desses últimos por uma codificação não-extensional (essa é uma razão para a introdução de uma contrapartida formal da exponenciação como primitiva).

\subsection{Definição de pertinência numérica.}

a) Escreveremos $\operatorname{ESC}(x, y) \approx z$ no lugar de

$$
\exists u \leqslant y \exists w<\overline{2}^{x}\left(\left(y=\left(\left(\overline{2}^{f_{1}^{1} x} \cdot u\right)+\left(\overline{2}^{x} \cdot z\right)+w\right)\right) \wedge z \leqslant \overline{1}\right) ;{ }^{(1)}
$$

b) $x \dot{\epsilon} y$ no lugar de $\operatorname{ESC}(x, y) \approx \overline{1}$.

Podemos dizer, então, que $n$ é um elemento de $m$ ou que $n$ é uma escolha em $m$ se $\langle n, m\rangle \in|x \dot{\epsilon} y|$.

Notemos, então, que 0 não é uma escolha em (ou um elemento de) 10, ou seja, que $\langle 0,10,0\rangle \in|\operatorname{ESC}(x, y) \approx z|$; isso porque $\mathbb{N} \vDash \operatorname{ESC}(\overline{0}, \overline{10}) \approx \overline{0}$, uma vez que, tomando $\overline{5}$ como $u$ e $\overline{0}$ como $w$, temos que $\mathbb{N} \leqslant \overline{5} \leqslant \overline{10} \wedge \overline{0}<\overline{2}^{0} \wedge \overline{0} \leqslant \overline{1} \wedge \overline{10}=\left(\overline{2}^{\overline{1}} \cdot \overline{5}\right)+\left(\overline{2}^{\overline{0}} \cdot \overline{0}\right)+\overline{0}$.

1. A diferença entre $\operatorname{ESC}(x, y) \approx z$ e a fórmula empregada por Hájek \& Pudlak [1998, p. 38] é a "adição" da subfórmula $z \leqslant \overline{1}$ (o que, nesse contexto, é apenas uma conveniência). 
Similarmente:

1) $\mathbb{N} \vDash \overline{10}=\left(\overline{2}^{2} \cdot \overline{2}\right)+\left(\overline{2}^{\overline{1}} \cdot \overline{1}\right)+\overline{0}$ e, assim, $\mathbb{N} \vDash \operatorname{ESC}(\overline{1}, \overline{10}) \approx \overline{1}$; de modo que 1 é uma escolha em (ou um elemento de) 10;

2) $\mathbb{N} \vDash \overline{10}=\left(\overline{2}^{\overline{3}} \cdot \overline{1}\right)+\left(\overline{2}^{\overline{2}} \cdot \overline{0}\right)+\overline{2} \mathrm{e}$, assim, $\mathbb{N} \vDash \operatorname{ESC}(\overline{2}, \overline{10}) \approx \overline{0}$;

3) $\mathbb{N} \vDash \overline{10}=\left(\overline{2}^{\overline{4}} \cdot \overline{0}\right)+\left(\overline{2}^{\overline{3}} \cdot \overline{1}\right)+\overline{2} \mathrm{e}$, assim, $\mathbb{N} \vDash \operatorname{ESC}(\overline{3}, \overline{10}) \approx \overline{1}$; de modo que 3 é uma escolha em (ou um elemento de) 10;

4) $\mathbb{N} \vDash \overline{10}=\left(\overline{2}^{\overline{5}} \cdot \overline{0}\right)+\left(\overline{2}^{\overline{4}} \cdot \overline{0}\right)+\overline{10}$ e, portanto, $\mathbb{N} \vDash \operatorname{ESC}(\overline{4}, \overline{10}) \approx \overline{0}$;

5) $\mathbb{N} \vDash \overline{10}=\left(\overline{2}^{\overline{6}} \cdot \overline{0}\right)+\left(\overline{2}^{\overline{5}} \cdot \overline{0}\right)+\overline{10} \mathrm{e}$, portanto, $\mathbb{N} \vDash \operatorname{ESC}(\overline{5}, \overline{10}) \approx \overline{0}$

Não é difícil notar, portanto, que apenas 1 e 3 são "escolhas" em (ou pertencem a) 10, ou seja, que $|x \dot{\epsilon} \overline{10}|=\{1,3\}$.

A fórmula $x \dot{\epsilon} y$ é essencialmente uma contrapartida da formal de uma interpretação, proposta inicialmente por Ackermann, dos conjuntos (puros) finitamente gerados no conjuntos números naturais.

A ideia subjacente é a seguinte: seja $k_{n} \ldots k_{1} k_{0}$ a representação binária de $m$ ( $k_{0}=$ '0' ou $k_{0}=$ ' 1 ', $k_{1}=$ '0' ou $k_{1}=$ ' 1 ', etc. $), i \in m$ sse $k_{i}=$ ' 1 '. Temos, assim, que a representação binária de 10 é '1010' e, portanto, que $|x \dot{\in} \overline{10}|=\{1,3\}$; que a representação binária de 23 é '10111' e, portanto, $|x \in \overline{23}|=\{0,1,2,4\}$ [cf., por exemplo, Kaye \& Wong, 2007, pp. 499-500].

\subsection{Alguns fatos básicos sobre a pertinência numérica.}

a) $|\operatorname{ESC}(x, y) \approx z|$ é o gráfico de uma função binária cuja imagem é $\{0,1\}$;

b) O número natural $m$ é a codificação do conjunto dos números naturais $n$ tais que $n \in|\operatorname{ESC}(x, \bar{m}) \approx \overline{1}|$ ou tais que $n \in|x \dot{\epsilon} \bar{m}| ;$

c) Para qualquer número natural $m$, se $n \in|x \dot{\epsilon} \bar{m}|$, então $n$ é menor do que $m$;

d) Além disso, $|x \dot{\epsilon} y|$ é extensional, ou seja, se, para qualquer $a, a \in|x \dot{\epsilon} \bar{n}|$ se e somente se $a \in|x \dot{\epsilon} \bar{m}|$, então $n$ e $m$ são iguais. 


\subsection{Definição de alguns conceitos conjuntistas básicos.}

a) Escreveremos $x \dot{\subseteq} y$ no lugar de $\forall z<x(z \dot{\in} x \rightarrow z \dot{\epsilon} y)$;

b) $\operatorname{MAX}(x) \approx y$ no lugar de $(y \dot{\epsilon} x \wedge \forall z<x(z \dot{\epsilon} x \rightarrow z<y)) \vee(y=0 \wedge x=0)$;

c) $[0, x] \approx y$ no lugar de $f_{1}^{1} y=2^{x+1}$;

d) $[0, x) \approx y$ no lugar $\operatorname{de} f_{1}^{1} y=2^{x}$;

e) $\{x\}$ no lugar de $2^{x}$;

f) $\{x, y\}$ nolugar de $2^{x}+2^{y}$;

g) $\operatorname{SEG}(x) \approx y$ no lugar de $\exists z<x(\operatorname{MAX}(x) \approx z \wedge[0, z] \approx y)$;

h) $\operatorname{RES}(x, y, z)$ no lugar de $x<2^{z} \wedge \forall w<z(w \dot{\epsilon} y \leftrightarrow w \dot{\epsilon} x)$.

Temos, assim, que:

a) $x \subseteq$ ㄷ é uma contrapartida formal $\Sigma_{0}$ de " $x$ é um subconjunto de $y$ ";

b) $\operatorname{MAX}(x) \approx y$ é uma contrapartida (formal $\Sigma_{0}$ ) de " $y$ é o maior elemento de $x$ ";

c) $[0, x] \approx y$ é uma contrapartida de " $y$ é o conjunto dos números naturais menores ou iguais a $x^{\prime \prime}$;

d) $[0, x) \approx y$ é uma contrapartida de " $y$ é o conjunto dos números menores que $x$ ";

e) $\{x\}$ é uma contrapartida de "o conjunto cujo único elemento é $x$ ";

f) $\{x, y\}$ é uma contrapartida de "o conjunto cujos dois únicos elementos são $x$ e $y$ ";

g) $\operatorname{SEG}(x) \approx y$ é uma contrapartida de " $y$ é o conjunto de todos os números menores ou iguais ao maior elemento de $x^{\prime \prime}$;

h) $\operatorname{RES}(x, y, z)$ é uma contrapartida de " $x$ é a restrição de $y$ aos elementos menores que $z$.

Notamos, anteriormente, que $|x \dot{\in} \overline{10}|=\{1,3\}$; agora, por $3 \mathbf{3 f}),\{\overline{1}, \overline{3}\}$ é, diferentemente de $\{1,3\}$, um termo de $L_{\text {P.A. }}$; mais especificamente, $\{\overline{1}, \overline{3}\}$ é um termo tal que $|x \in\{\overline{1}, \overline{3}\}|=\{1,3\}$. 


\subsection{Definição de alguns conceitos conjuntistas (continuação).}

a) Escreveremos $\langle x, y\rangle$ nolugar de $\{\{x\},\{x, y\}\}$;

b) $x \times y \approx z$ no lugar de $\forall w<x \forall v<y(w \dot{\in} x \wedge v \dot{\in} y \rightarrow\langle w, v\rangle \dot{\in} z) \wedge \forall u<z(u \dot{\epsilon} z \rightarrow$ $\exists w<x \exists v<y(w \dot{\in} x \wedge v \dot{e} y \wedge\langle w, v\rangle=u))$

C) $x \dot{\operatorname{DOM}}(y)$ no lugar de $\exists z<y(\langle x, z\rangle \dot{\epsilon} y)$;

d) $x \dot{\operatorname{RAN}}(y)$ no lugar de $\exists z<y(\langle z, x\rangle \dot{\in} y)$;

e) $\operatorname{REL}(x)$ no lugar de $\forall w<x(w \in \dot{x} \rightarrow \exists u<w \exists v<w(\langle u, v\rangle=w))$;

f) $\operatorname{MAP}(x)$ no lugar de

$\operatorname{REL}(x) \wedge \forall v<x \forall u<x \forall w<x(\langle v, u\rangle \dot{\in} z \wedge\langle v, w\rangle \dot{\in} z \rightarrow u=w))$.

Temos, agora, que:

a) $\langle x, y\rangle$ é uma contrapartida (formal $\Sigma_{0}$ ) de "o par ordenado formado por $x$ e $y$ ";

b) $x \times y \approx z$ é uma contrapartida de " $z$ é o produto cartesiano de $x$ e $y$ ";

c) $x \in \dot{\operatorname{DOM}}(y)$ é uma contrapartida de " $x$ é um elemento do domínio de $y$ ";

d) $x \dot{\epsilon} \operatorname{RAN}(y)$ é uma contrapartida de " $x$ é um elemento do contradomínio de $y$ ";

e) $\operatorname{REL}(x)$ é uma contrapartida de " $x$ é uma relação";

f) $\operatorname{MAP}(x)$ é uma contrapartida de " $x$ é um mapa".

\subsection{Definição de alguns conceitos da teoria das sequências finitas.}

a) Escreveremos $\operatorname{COMP}(x) \simeq y$ no lugar de $\forall w<x\left(w \dot{\operatorname{DOM}}(x) \leftrightarrow \exists v<x\left(w \dot{\epsilon} v \wedge\left[0, f_{1}^{1} y\right) \approx v\right)\right) \wedge \operatorname{MAP}(x) ;$

b) $\operatorname{SEQ}(x)$ no lugar de $\exists y<x(\operatorname{COMP}(x) \simeq y)$;

c) $(x)_{y+1} \simeq z$ no lugar de $\langle y, z\rangle \dot{\epsilon} x$;

d) $(x)_{1} \simeq y$ no lugar de $\langle\overline{0}, y\rangle \dot{\in} x$;

e) $(x)_{2} \simeq y$ no lugar de $\langle\overline{1}, y\rangle \dot{\in} x$;

f) $x \frown\langle y\rangle \simeq z$ no lugar de $\operatorname{SEQ}(x) \rightarrow \forall w<z(w \dot{\epsilon} z \leftrightarrow \exists v<x(w \dot{\epsilon} v \vee\langle\operatorname{COMP}(x), y\rangle=w))$. 
Temos, finalmente, que:

a) $\operatorname{COMP}(x) \simeq y$ é uma contrapartida (formal $\Sigma_{0}$ ) de " $y$ é o comprimento de $x$, se $x$ for uma sequência";

b) $\operatorname{SEQ}(x)$ é uma contrapartida de " $x$ é uma sequência";

c) $(x)_{y+1} \simeq z$ é uma contrapartida de " $z$ é o $y+1$-ésimo membro de $x$ (se $x$ for uma sequência de comprimento adequado)";

d) $(x)_{1} \simeq y$ é uma contrapartida de " $y$ é o primeiro membro de $x$ ";

e) $(x)_{2} \simeq y$ é uma contrapartida de " $y$ é o segundo membro de $x$ ";

f) $x \frown\langle y\rangle \simeq z$ é uma contrapartida de " $z$ é a concatenação entre $x$ e $y$ ".

\section{§4. Introdução à gödelização da sintaxe 2: termos}

As práticas mais comuns de gödelização dos conceitos metateóricos estabelecem ou (1) fórmulas $\Sigma_{0}$ de alguma extensão da linguagem da aritmética [cf., por exemplo, Feferman, 1960, p. 52] ou (2) tanto fórmulas $\Sigma_{1}$ quanto fórmulas $\Pi_{1}$ em $L_{\text {P.A. como }}$ contrapartidas formais dos termos de uma linguagem dada (de modo que o conjunto de números de Gödel dos termos seja $\left.\Delta_{1}\right)$. Apresentaremos, abaixo, uma fórmula $\Sigma_{0}$ em

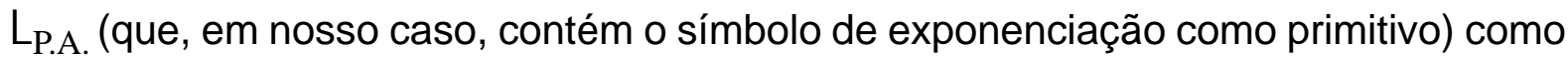
contrapartida formal do conjunto dos termos das linguagens de base.

\subsection{Definição da contrapartida formal do conjunto de numerais.}

a) Escreveremos $\mathrm{CX}(x) \approx y$ no lugar de $\forall x_{1} \leqslant y\left(f_{1}^{1} x_{1}=y \rightarrow \overline{10} x_{1} \leqslant x\right) \wedge\left(f_{1}^{1} x \leqslant \overline{10} y\right)$;

b) $\operatorname{SUC}(x) \approx y$ no lugar de $\exists x_{2} \leqslant y\left(\mathrm{CX}(x) \approx x_{2} \wedge\left(x+\left(\overline{110} \cdot \overline{10} x_{2}\right)\right)=y\right)$;

c) $\operatorname{LIM}(x)$ no lugar de $f_{3}^{2} \overline{2} f_{3}^{2} \overline{2} f_{3}^{2} \overline{2}(x+\overline{2})$;

d) $\mathrm{NU}(x)$ no lugar de $\exists y_{1} \leqslant \operatorname{LIM}(x) \exists x_{7}<y_{1}\left(\operatorname{SEQ}\left(y_{1}\right) \wedge \operatorname{COMP}\left(y_{1}\right) \simeq f_{1}^{1} x_{7} \wedge\right.$ $\forall x_{6} \leqslant x_{7} \exists x_{5}<x_{6}\left(\left(y_{1}\right)_{x_{6}} \simeq \overline{10} \vee \exists x_{4}<y_{1} \exists x_{3}<y_{1}\left(\left(y_{1}\right)_{x_{6}} \simeq x_{4} \wedge\left(y_{1}\right)_{x_{5}} \simeq x_{3} \wedge \operatorname{SUC}\left(x_{3}\right) \approx x_{4}\right)\right) \wedge$ $\left(y_{1}\right)_{x_{7}} \simeq x$ 
No caso, $|\mathrm{CX}(x) \approx y|(n)$ é o comprimento da representação decimal de $n$ ou, nos casos metateoricamente relevantes, $|\mathrm{CX}(x) \approx y|(n)$ é a complexidade da expressão de $L_{P . A .}$ cujo número de Gödel é $n$ [quanto à notação de barra simples cf. p. 46].

$|\operatorname{SUC}(x) \approx y|(n)$ é, por sua vez, o número de Gödel da concatenação entre ' $f_{1}^{1}$ ' (ou seja, do nome da operação de sucessão) e a expressão cujo número de Gödel é $n$.

$|\mathrm{NU}(x)|$ é, então, o conjunto dos números de Gödel dos numerais de $\mathrm{L}_{\mathrm{P} . \mathrm{A}}$.

Grosso modo, $n \in|\mathrm{NU}(x)|$ se existe uma sequência menor que $|\operatorname{LIM}(x) \approx y|(n)$ cujo último elemento é $n$ e tal que cada um dos seus elementos é (1) o número de Gödel da constante ' $c_{1}$ ' ou (2) é o número de Gödel da concatenação entre ' $f_{1}^{1}$ ' e um numeral cujo número de Gödel aparece anteriormente na sequência.

Uma notação semi-formal e mais humana, na qual as contrapartidas formais dos gráficos de funções totais e parciais (as condições de comutação estarão pressupostas $\left.^{(2)}\right)$ são apresentadas como termos, talvez seja esclarecedora e possibilite uma mediação entre as contrapartidas formais desejadas e as razões "informais" que as sustentam como tal.

Uma versão semi-formal de $\mathrm{NU}(x)$ poderia ser, então: $\exists s \leqslant \operatorname{LIM}(x)(\operatorname{SEQ}(s) \wedge$ $\left.\forall w \leqslant \operatorname{COMP}(s)\left((s)_{w}=\overline{10} \vee \exists v<w\left((s)_{w}=\operatorname{SUC}\left((s)_{v}\right)\right)\right) \wedge(s)_{\operatorname{COMP}(s)}=x\right)$.

De modo que, a fórmula abaixo é, por assim dizer, a condição imposta às sequências de construção dos numerais formais:

$$
\forall w \leqslant \operatorname{COMP}(s)\left((s)_{w}=\overline{10} \vee \exists v<w\left((s)_{w}=\operatorname{SUC}\left((s)_{v}\right)\right) .\right.
$$

Grosso modo, ' $(s)_{w}=\overline{10}$ ' estabelece como tais sequências devem "começar", enquanto ' $\exists v<w\left((s)_{w}=\mathrm{SUC}\left((s)_{v}\right)\right.$ ' é uma "condição de prosseguimento" dessas sequências.

2. Portanto, escreveremos, por exemplo e mais sucintamente, $\operatorname{SEQ}(x) \wedge \forall w \leqslant \operatorname{COMP}(x)\left(\mathrm{NU}\left((x)_{w}\right)\right)$ no lugar de $\operatorname{SEQ}(x) \wedge \exists z \leqslant x\left(\operatorname{COMP}(x) \simeq z \wedge \forall w \leqslant z \exists y \leqslant x\left((x)_{w} \simeq y \wedge \mathrm{NU}(y)\right)\right.$ que é uma contrapartida de " $x$ é uma sequência finita de numerais"; notemos que $\operatorname{SEQ}(x)$ é uma condição suficiente para que exista um e somente um $z$ tal que $\operatorname{COMP}(x) \simeq z$ e, por sua vez, isso é uma condição suficiente para que, para todo $w$ menor ou igual a $z$, exista um e somente um $y$ tal que $(x)_{w} \simeq y$. 
No caso da condição acima em particular e de condições fundamentadas no conceito de concatenação em geral, é possível estabelecer um limite "exponencial" para as sequências de formação relevantes. Desse modo, teríamos uma contrapartida formal imediata da decidibilidade dos conjuntos correspondentes (por meio da definição de $\mathbb{N}$ e dos algoritmos escolares). O limite acima foi estabelecido por meio do estudo das possíveis sequências de construção de termos (os detalhes não são importantes aqui) e, embora péssimo em termos computacionais, é mais do que suficiente para nossos propósitos teóricos imediatos.

\subsection{Definição de algumas abreviações auxiliares.}

a) Escreveremos $x * y \approx z$ no lugar de $\exists x_{2} \leqslant z\left(\mathrm{CX}(y) \approx x_{2} \wedge\left(y+\left(x \cdot \overline{10} x_{2}\right)\right)=z\right)$;

b) $x * y * z \approx w$ no lugar de

$$
\exists x_{4} \leqslant w \exists x_{3} \leqslant x_{4}\left(y * z \approx x_{4} \wedge \mathrm{CX}\left(x_{4}\right) \approx x_{3} \wedge\left(x_{4}+x \cdot \overline{10} x_{3}\right)=z\right) ;
$$

c) $\dot{+}(x, y) \approx z$ no lugar de $\overline{1110} * x * y \approx z$;

d) $:(x, y) \approx z$ no lugar de $\overline{11100} * x * y \approx z$;

e) $\operatorname{EXP}(x, y) \approx z$ no lugar de $\overline{111000} * x * y \approx z$

Temos, portanto, contrapartidas formais $\Sigma_{0}$, respectivamente, da concatenação entre 2 e 3 expressões, e da formação de termos.

Apesar da relativa simplicidade das fórmulas acima, a contrapartida formal do conjunto de termos fechados é praticamente ilegível:

\subsection{Definição da contrapartida formal do conjunto de termos fechados.}

a) Escreveremos $\operatorname{TF}(x)$ no lugar de $\exists y_{1} \leqslant \operatorname{LIM}(x) \exists x_{11}<y_{1}\left(\operatorname{SEQ}\left(y_{1}\right) \wedge\right.$ $\operatorname{COMP}\left(y_{1}\right) \simeq f_{1}^{1} x_{11} \wedge \forall x_{10} \leqslant x_{11} \exists x_{9}<x_{10} \exists x_{8}<x_{10}\left(\left(y_{1}\right)_{x_{10}} \simeq \overline{10} \vee \exists x_{4}<y_{1} \exists x_{3}<y_{1}\left(\left(y_{1}\right)_{x_{10}} \simeq x_{4}\right.\right.$ $\left.\wedge\left(y_{1}\right)_{x_{9}} \simeq x_{3} \wedge \operatorname{SUC}\left(x_{3}\right) \approx x_{4}\right) \vee \exists x_{7}<y_{1} \exists x_{6}<y_{1} \exists x_{5}<y_{1}\left(\left(y_{1}\right)_{x_{10}} \simeq x_{7} \wedge\left(y_{1}\right)_{x_{9}} \simeq x_{6} \wedge\left(y_{1}\right)_{x_{8}} \simeq x_{5}\right.$ $\left.\wedge \dot{+}\left(x_{5}, x_{6}\right) \approx x_{7}\right) \vee \exists x_{7}<y_{1} \exists x_{6}<y_{1} \exists x_{5}<y_{1}\left(\left(y_{1}\right)_{x_{10}} \simeq x_{7} \wedge\left(y_{1}\right)_{x_{9}} \simeq x_{6} \wedge\left(y_{1}\right)_{x_{8}} \simeq x_{5}\right.$ $\left.\wedge:\left(x_{5}, x_{6}\right) \approx x_{7}\right) \vee \exists x_{7}<y_{1} \exists x_{6}<y_{1} \exists x_{5}<y_{1}\left(\left(y_{1}\right)_{x_{10}} \simeq x_{7} \wedge\left(y_{1}\right)_{x_{9}} \simeq x_{6} \wedge\left(y_{1}\right)_{x_{8}} \simeq x_{5} \wedge\right.$ $\left.\left.\operatorname{EXP}\left(x_{5}, x_{6}\right) \approx x_{7}\right)\right) \wedge\left(y_{1}\right)_{x_{11}} \simeq x$ 
Muito mais simplesmente, escreveremos uma versão semi-formal de $\mathrm{TF}(x)$ cuja forma é:

$\exists s \leqslant \operatorname{LIM}(x)\left(\operatorname{SEQ}(s) \wedge \forall w \leqslant \operatorname{COMP}(s)\left(\alpha_{1} \vee \alpha_{2} \vee \alpha_{3} \vee \alpha_{4} \vee \alpha_{5}\right) \wedge(s)_{\operatorname{COMP}(s)}=x\right)$

E na qual as subfórmulas $\alpha_{1}, \alpha_{2}, \alpha_{3}, \alpha_{4}$ e $\alpha_{5}$ são, respectivamente:

$(s)_{w}=\overline{10}$;

$\exists v<w\left((s)_{w}=\operatorname{SUC}\left((s)_{v}\right)\right)$

$\exists v_{1}<w \exists v_{2}<w\left((s)_{w}=\dot{+}\left((s)_{v_{1}},(s)_{v_{2}}\right)\right)$

$\exists v_{1}<w \exists v_{2}<w\left((s)_{w}=:\left((s)_{v_{1}},(s)_{v_{2}}\right)\right)$;

$\left.\exists v_{1}<w \exists v_{2}<w\left((s)_{w}=\operatorname{EXP}\left((s)_{v_{1}},(s)_{v_{2}}\right)\right)\right)$.

Ou seja, $\alpha_{1}$ é a contrapartida formal da base da sequência de formação dos termos fechados, enquanto $\alpha_{2}, \alpha_{3}, \alpha_{4}$ e $\alpha_{5}$ são as "condições de prosseguimento".

Uma vez que a leitura da grande maioria das próximas contrapartidas formais é impraticável, deveremos empregar descrições "informais" ou "semi-formais" dessas fórmulas. Entretanto, é importante notar que, munidos da paciência de Jó, poderíamos transformar tais descrições informais nas respectivas contrapartidas formais.

\subsection{Descrição da contrapartida formal do conjunto de termos (em geral).}

a) Escreveremos $\operatorname{VAR}(x)$ no lugar de $\exists x_{1}<x\left(\overline{30} \cdot \overline{10} x_{1}=x\right)$;

b) $\operatorname{TM}(x)$ no lugar de $\exists s \leqslant \operatorname{LIM}(x)\left(\operatorname{SEQ}(s) \wedge \forall w \leqslant \operatorname{COMP}(s)\left(\alpha_{1} \vee \operatorname{VAR}\left((s)_{w}\right) \vee \alpha_{2} \vee\right.\right.$ $\left.\left.\alpha_{3} \vee \alpha_{4} \vee \alpha_{5}\right) \wedge(s)_{\operatorname{COMP}(s)}=x\right)$.

A diferença entre $\operatorname{TM}(x)$ e $\operatorname{TF}(x)$ é a introdução da cláusula, $\operatorname{VAR}\left((s)_{w}\right)$, relativa às variáveis das linguagens de base. 


\section{§5. Introdução à gödelização da semântica: fórmulas-legenda}

As contrapartidas formais de sequências do tipo $\langle\overline{0}, 0\rangle,\langle\overline{1}, 1\rangle,\langle\overline{1}+\overline{0}, 1\rangle, \ldots,\langle\tau$, denotação de $\tau\rangle$, nas quais os termos fechados são "construídos" ao mesmo tempo em que os valores correspondentes são “calculados”, fundamentarão nossa gödelização do conceito de denotação de termos por números.

Suponhamos, por um momento, que a fórmula-legenda para termos fechados ou, abreviadamente, $\operatorname{LTF}(x) \simeq y$ tenha sido descrita adequadamente por meio de sequências

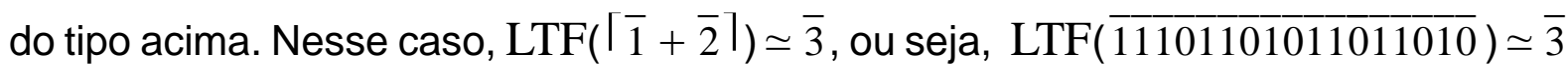
seria estabelecida pela sequência $\langle\overline{0}, 0\rangle,\langle\overline{1}, 1\rangle,\langle\overline{2}, 2\rangle,\langle\overline{1}+\overline{2}, 3\rangle$.

Por motivos que não explicaremos, entretanto, nenhum dos termos das linguagens de base seria capaz de fornecer um limite para esse tipo de sequência; tal limite seria super-exponencial ${ }^{(3)}$. Portanto, as fórmulas-legenda serão, por razões técnicas, introduzidas tanto como fórmulas $\Sigma_{1}$ quanto como fórmulas $\Pi_{1}{ }^{(4)}$

\subsection{Descrição semi-formal da fórmula-legenda $\Sigma_{1}$.}

a) Escreveremos $\operatorname{LTF}^{\Sigma}(x) \simeq y$ no lugar de $\exists s\left(\operatorname{SEQ}(s) \wedge \forall w \leqslant \operatorname{COMP}(s)\left(\alpha_{1} \vee \alpha_{2} \vee\right.\right.$ $\left.\left.\alpha_{3} \vee \alpha_{4} \vee \alpha_{5}\right) \wedge x=\left((s)_{\mathrm{COMP}(s)}\right)_{1} \wedge y=\left((s)_{\mathrm{COMP}(s)}\right)_{2}\right) ;$

b) Onde $\alpha_{1}$ está no lugar de $(s)_{w}=\langle\overline{10}, \overline{0}\rangle$ (ou seja, de $(s)_{w}=\langle[\overline{0}], \overline{0}\rangle$ );

c) $\alpha_{2}$ no lugar de $\exists v<w\left((s)_{w}=\left\langle\operatorname{SUC}\left(\left((s)_{v}\right)_{1}\right), f_{1}^{1}\left((s)_{v}\right)_{2}\right\rangle\right)$;

d) $\alpha_{3}$ no lugar de $\exists v_{1}<w \exists v_{2}<w\left((s)_{w}=\left\langle\dot{+}\left(\left((s)_{v_{1}}\right)_{1},\left((s)_{v_{2}}\right)_{1}\right),\left((s)_{v_{1}}\right)_{2}+\left((s)_{v_{2}}\right)_{2}\right\rangle\right)$;

e) $\alpha_{4}$ no lugar de $\exists v_{1}<w \exists v_{2}<w\left((s)_{w}=\left\langle\cdot\left(\left((s)_{v_{1}}\right)_{1},\left((s)_{v_{2}}\right)_{1}\right),\left((s)_{v_{1}}\right)_{2} \cdot\left((s)_{v_{2}}\right)_{2}\right\rangle\right)$;

f) $\alpha_{5}$ no lugar de $\exists v_{1}<w \exists v_{2}<w\left((s)_{w}=\left\langle\operatorname{EXP}\left(\left((s)_{v_{1}}\right)_{1},\left((s)_{v_{2}}\right)_{1}\right), f_{3}^{2}\left((s)_{v_{1}}\right)_{2}\left((s)_{v_{2}}\right)_{2}\right\rangle\right)$.

3. A introdução de uma função "super-exponencial" como primitiva não seria de ajuda, uma vez que nosso limite se tornaria, por sua vez, "super-super-exponencial", etc..

4. O que, na verdade, é mais usual, mas cuja necessidade é às vezes escamoteada por um procedimento generalizado de introdução dos conceitos metateóricos recursivos necessários à argumentação. Devemos notar, então, que todas as funções e relações definidas por fórmulas $\Sigma_{0}$ são recursivas primitivas, mas nem todas as funções e relações recursivas primitivas são $\Sigma_{0}$ [cf. nota 5 do Cap. II p. 49]; quais as relações recursivas primitivas são, além disso, $\Sigma_{0}$ depende dos termos que estão à disposição em nossa linguagem. 
Desse modo, as sequências $s$ serão sequências de pares ordenados, nas quais o primeiro elemento de cada par é um termo fechado $\tau$ de $L_{P . A . ~}$ e o segundo a denotação de $\tau$; as subfórmulas $\alpha_{2}, \alpha_{3}, \alpha_{4}$ e $\alpha_{5}$ são "condições de prosseguimento", respectivamente, para ' $f_{1}^{1}$, ' '+', ‘’’ ' ' $f_{3}^{2}$ ' (símbolos da sucessão, adição, multiplicação e exponenciação), enquanto $\alpha_{1}$ determina uma denotação para ' $c_{1}$ ' (ou seja, para $\overline{0}$ ).

\subsection{Descrição da fórmula-legenda $\Pi_{1}$.}

a) Escreveremos $\operatorname{LTF}^{\Pi}(x) \simeq y$ no lugar de $\forall s\left(\operatorname{SEQ}(s) \wedge \forall w \leqslant \operatorname{COMP}(s)\left(\alpha_{1} \vee \alpha_{2} \vee\right.\right.$ $\left.\left.\left.\alpha_{3} \vee \alpha_{4} \vee \alpha_{5}\right) \wedge x=\left((s)_{\operatorname{COMP}(s)}\right)_{1}\right) \rightarrow y=\left((s)_{\operatorname{COMP}(s)}\right)_{2}\right)$.

Seria possível descrevermos (e iremos supor que o fizemos) as fórmulas $\operatorname{LTM}^{\Sigma}(x, y) \simeq z \operatorname{eLTM}^{\Pi}(x, y) \simeq z$ como contrapartidas formais $\Sigma_{1}$ e $\Pi_{1}$ de " $z$ é a denotação do termo $x$ segundo $y$ ", onde $y$ é uma atribuição de valores às variáveis de $x$.

A ideia subjacente à descrição poderia ser: seja $e$ uma função do conjunto dos termos no conjunto de variáveis de $L_{\text {P.A. }}$ tal que $e(\tau)$ é a variável de maior índice de $\tau$; seja $\left\langle k_{1}, \ldots, k_{n}\right\rangle$ uma sequência de números naturais; desse modo, a denotação de $\tau$, segundo $\left\langle k_{1}, \ldots, k_{n}\right\rangle$, seria $d\left(\tau v_{1} / \bar{k}_{1} v_{2} / \bar{k}_{2} \ldots v_{n} / \bar{k}_{n} v_{n+1} / \bar{k}_{n} \ldots e(\tau) / \bar{k}_{n}\right)$.

\section{§6. Introdução à gödelização da sintaxe 3: sentenças}

Introduziremos, nessa seção, contrapartidas $\Sigma_{0}$ de inúmeros conceitos metateóricos relacionados aos conceitos de fórmula e sentença das linguagens de base. Por razões "mnemônicas", essas serão acompanhadas por uma série de explicações, por assim dizer, intuitivas e de exemplos.

\subsection{Descrição de algumas fórmulas auxiliares.}

a) Escreveremos $\doteq(x, y) \approx z$ no lugar de $\overline{220} * x * y \approx z$;

b) $\dot{\leqslant}(x, y) \approx z$ no lugar de $\overline{2200} * x * y \approx z$; 
c) $\doteq(x)$ no lugar de $\exists x_{13}<x \exists x_{12}<x\left(\overline{220} * x_{12} * x_{13} \approx x \wedge \mathrm{TM}\left(x_{12}\right) \wedge \mathrm{TM}\left(x_{13}\right)\right)$;

d) $\dot{\leqslant}(x)$ no lugar de $\exists x_{13}<x \exists x_{12}<x\left(\overline{2200} * x_{12} * x_{13} \approx x \wedge \operatorname{TM}\left(x_{12}\right) \wedge \operatorname{TM}\left(x_{13}\right)\right)$;

e) $\mathrm{PP} \doteq(x) \simeq y$ no lugar de $\exists x_{12}<x\left(\overline{220} * y * x_{12} \approx x \wedge \mathrm{TM}(y) \wedge \mathrm{TM}\left(x_{12}\right)\right)$;

f) $\mathrm{PS} \doteq(x) \simeq y$ no lugar de $\exists x_{12}<x\left(\overline{220} * x_{12} * y \approx x \wedge \mathrm{TM}\left(x_{12}\right) \wedge \mathrm{TM}(y)\right)$;

g) $\mathrm{PP} \dot{\leqslant}(x) \simeq y$ no lugar de $\exists x_{12}<x\left(\overline{2200} * y * x_{12} \approx x \wedge \operatorname{TM}(y) \wedge \operatorname{TM}\left(x_{12}\right)\right)$;

h) $\mathrm{PS} \leqslant(x) \simeq y$ no lugar de $\exists x_{12}<x\left(\overline{2200} * x_{12} * y \approx x \wedge \operatorname{TM}\left(x_{12}\right) \wedge \operatorname{TM}(y)\right)$;

i) $\mathrm{SENT} \doteq(x)$ no lugar de $\exists x_{13}<x \exists x_{12}<x\left(\overline{220} * x_{12} * x_{13} \approx x \wedge \mathrm{TF}\left(x_{12}\right) \wedge \mathrm{TF}\left(x_{13}\right)\right)$;

j) $\mathrm{SENT} \leqslant(x)$ no lugar de $\exists x_{13}<x \exists x_{12}<x\left(\overline{2200} * x_{12} * x_{13} \approx x \wedge \mathrm{TF}\left(x_{12}\right) \wedge \mathrm{TF}\left(x_{13}\right)\right)$.

$|\dot{=}(x, y) \approx z| \mathrm{e}|\dot{\leqslant}(x, y) \approx z|$ são gráficos de funções tais que se $i$ e $j$ são números de Gödel, respectivamente, dos termos $\tau_{i}$ e $\tau_{j}$, então $|\doteq(x, y) \approx z|(i, j)$ será o número de Gödel de $\tau_{i}=\tau_{j}$ e $|\dot{\leqslant}(x, y) \approx z|(i, j)$ será $g\left(\tau_{i} \leqslant \tau_{j}\right)$.

$|\doteq(x)|=\| x$ é uma equação $\|\mathrm{e}|\dot{\xi}(x)|=\| x$ é uma inequação $\|$.

$|\mathrm{PP} \dot{=}(x) \simeq y|=\| x$ é uma equação cujo primeiro termo é $y \|$;

$|\mathrm{PS} \doteq(x) \simeq y|=\| x$ é uma equação cujo segundo termo é $y \|$;

$|\mathrm{PP} \dot{\leqslant}(x) \simeq y|=\| x$ é uma inequação cujo primeiro termo é $y \|$;

$|\mathrm{PS} \dot{\leqslant}(x) \simeq y|=\| x$ é uma inequação cujo segundo termo é $y \|$.

Finalmente, $|\operatorname{SENT} \doteq(x)|=\| x$ é uma equação sem variáveis livres $\|$;

e $|\operatorname{SENT} \dot{\leqslant}(x)|=\| x$ é uma inequação sem variáveis livres $\|$.

\subsection{Descrição da contrapartida formal dos conjuntos das fórmulas e das} sentenças atômicas de $L_{\text {P.A. }}$.

a) Escreveremos $\mathrm{FORM}_{0}(x)$ no lugar de $\dot{=}(x) \vee \dot{\leqslant}(x)$;

b) $\operatorname{SENT}_{0}(x)$ no lugar de $\operatorname{SENT} \doteq(x) \vee \operatorname{SENT} \dot{\leqslant}(x)$.

\subsection{Descrição de algumas fórmulas auxiliares.}

a) Escreveremos $\dot{\mathrm{V}}(x) \approx y$ no lugar de $\overline{20} * x \approx y$;

b) $\dot{\mathrm{F}}(x) \approx y$ no lugar de $\overline{200} * x \approx y$; 
c) $\dot{\mathrm{V}}(x)$ no lugar de $\exists x_{12}<x\left(\overline{20} * x_{12} \approx x \wedge \mathrm{TM}\left(x_{12}\right)\right)$;

d) $\dot{\mathrm{F}}(x)$ no lugar de $\exists x_{12}<x\left(\overline{200} * x_{12} \approx x \wedge \mathrm{TM}\left(x_{12}\right)\right)$;

e) $\mathrm{PV}(x) \simeq y$ no lugar de $\overline{20} * y \approx x \wedge \mathrm{TM}(y)$;

f) $\mathrm{P} \dot{\mathrm{F}}(x) \simeq y$ no lugar de $\overline{200} * y \approx x \wedge \mathrm{TM}(y)$;

g) $\operatorname{SENT\dot {V}}(x)$ no lugar de $\exists x_{12}<x\left(\overline{20} * x_{12} \approx x \wedge \mathrm{TF}\left(x_{12}\right)\right)$;

h) $\operatorname{SENTF}(x)$ no lugar de $\exists x_{12}<x\left(\overline{200} * x_{12} \approx x \wedge \operatorname{TF}\left(x_{12}\right)\right)$.

O paralelismo entre as fórmulas de 6.3 e as de 6.1 é evidente, e. $g .:|\dot{\mathrm{V}}(x) \approx y| \mathrm{e}$ um gráfico de função tal que se $i$ é um número de Gödel do termo $\tau$, então $|\dot{\mathrm{V}}(x) \approx y|(i)$ é o número de Gödel de $\mathrm{V}(\tau) ;|\dot{\mathrm{V}}(x)|=\|$ para algum termo $\tau$, $x$ é o número de Gödel de $\mathrm{V}(\tau) \|$.

\subsection{Descrição da contrapartida formal dos conjuntos das fórmulas} atômicas de $L_{\text {P.A.V. }} L_{\text {P.A.F. }}$ e $L_{\text {P.A.V.F. }}$.

a) Escreveremos FORM $(\mathrm{V})_{0}(x)$ no lugar de $\dot{=}(x) \vee \dot{\leqslant}(x) \vee \dot{\mathrm{V}}(x)$;

b) $\operatorname{FORM}(\mathrm{F})_{0}(x)$ no lugar de $\doteq(x) \vee \dot{\leqslant}(x) \vee \dot{\mathrm{F}}(x)$;

c) $\operatorname{FORM}(\mathrm{VF})_{0}(x)$ no lugar de $\doteq(x) \vee \dot{\leqslant}(x) \vee \dot{\mathrm{V}}(x) \vee \dot{\mathrm{F}}(x)$.

As contrapartidas formais das sentenças atômicas $L_{\text {P.A.V. }}$ L.A.F. $_{\text {P.A.V.F. }}$ seguem os mesmos princípios da cláusula 6.2.b), e.g.:

Escreveremos $\operatorname{SENT}(\mathrm{VF})_{0}(x)$ no lugar de $\operatorname{SENT} \doteq(x) \vee \operatorname{SENT} \dot{\doteq}(x) \vee \operatorname{SENTV}(x)$ $\vee \operatorname{SENT\dot {F}}(x)$.

\subsection{Descrição de algumas fórmulas auxiliares.}

a) Escreveremos $\dot{\sim}(x) \approx y$ no lugar de $\overline{4} * x \approx y$;

b) $\dot{\vee}(x, y) \approx z$ no lugar de $\overline{5} * x * y \approx z$;

c) $\dot{\lambda}(x, y) \approx z$ no lugar de $\overline{6} * x * y \approx z$;

d) $\dot{\forall}(x, y) \approx z$ no lugar de $\overline{7} * x * y \approx z$;

e) $\dot{\exists}(x, y) \approx z$ no lugar de $\overline{8} * x * y \approx z$. 
Novamente, o paralelismo é evidente entre as fórmulas de 6.5 e aquelas de 6.1.a-b) e 6.3.a-b), e. $g .:|\dot{\vee}(x, y) \approx z|$ é um gráfico tal que se $i$ e $j$ são números de Gödel, respectivamente, das fórmulas $\alpha$ e $\beta$, então $|\dot{\vee}(x, y) \approx z|(i, j)$ é o número de Gödel $\operatorname{de} \alpha \vee \beta$.

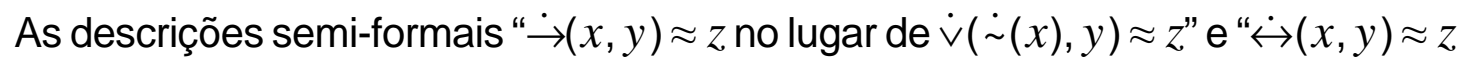
no lugar de $\dot{\wedge}(\dot{\rightarrow}(x, y), \dot{\rightarrow}(y, x)) \approx z$ " correspondem às definições do condicional e do bicondicional [cf. p. 43].

6.6. Descrição (semi-formal) das contrapartidas formais do conjunto das fórmulas de $L_{P . A}$ :

a) Escreveremos $\mathrm{FORM}(x)$ no lugar de $\exists s \leqslant \operatorname{LIM}(x)\left(\operatorname{SEQ}(s) \wedge \forall w \leqslant \operatorname{COMP}(s)\left(\alpha_{1}\right.\right.$ $\left.\left.\vee \alpha_{2} \vee \alpha_{3} \vee \alpha_{4} \vee \alpha_{5} \vee \alpha_{6}\right) \wedge(s)_{\operatorname{COMP}(s)}=x\right)$;

b) Onde $\alpha_{1}$ está no lugar de $\operatorname{FORM}_{0}\left((s)_{w}\right)$;

c) $\alpha_{2}$ no lugarde $\exists v<w\left((s)_{w}=\dot{\sim}\left((s)_{v}\right)\right)$;

d) $\alpha_{3}$ no lugar de $\exists v_{1}<w \exists v_{2}<w\left((s)_{w}=\dot{v}\left((s)_{v_{1}},(s)_{v_{2}}\right)\right)$;

e) $\alpha_{4}$ no lugar de $\exists v_{1}<w \exists v_{2}<w\left((s)_{w}=\dot{\wedge}\left((s)_{v_{1}},(s)_{v_{2}}\right)\right)$;

f) $\alpha_{5}$ no lugar de $\exists v_{1}<x \exists v_{2}<w\left(\operatorname{VAR}\left(v_{1}\right) \wedge(s)_{w}=\dot{\forall}\left(v_{1},(s)_{v_{2}}\right)\right)$;

g) $\alpha_{6}$ no lugar de $\exists v_{1}<x \exists v_{2}<w\left(\operatorname{VAR}\left(v_{1}\right) \wedge(s)_{w}=\dot{\exists}\left(v_{1},(s)_{v_{2}}\right)\right)$.

\subsection{Descrição das contrapartidas formais dos conjuntos de tipos de} fórmulas moleculares de $L_{P . A}$.

As contrapartidas formais dos conjuntos das negações, disjunções, conjunções e generalizações universais e existencias são introduzidas de maneira análoga à introdução de $\doteq(x) \mathrm{e} \dot{\leqslant}(x)$ (contrapartidas dos conjuntos de equações e inequações das linguagens de base). Exemplos:

a) Escreveremos $\dot{\wedge}(x)$ no lugar de:

$$
\exists t_{1}<x \exists t_{2}<x\left(\overline{6} * t_{1} * t_{2} \approx x \wedge \operatorname{FORM}\left(t_{1}\right) \wedge \operatorname{FORM}\left(t_{2}\right)\right)
$$

b) $\dot{\exists}(x)$ no lugar de $\exists t_{1}<x \exists t_{2}<x\left(\overline{8} * t_{1} * t_{2} \approx x \wedge \operatorname{VAR}\left(t_{1}\right) \wedge \operatorname{FORM}\left(t_{2}\right)\right)$ 
Iremos pressupor uma descrição $\Sigma_{0}$ adequada, $x(y / z) \simeq w$, da contrapartida formal de " $w$ é a substituição da variável livre $y$ por $z$ em $x$ " tal como essa operação foi definida em II 1.8 p. 43.

\subsection{Descrição das contrapartidas formais das projeções de subfórmulas.} As projeções (de única, de primeira e segunda subfórmulas) das fórmulas moleculares das linguagens de base, ou seja, as "operações" inversas das definições de $\mathbf{6 . 5}$ são introduzidas pela mesma técnica de $\mathrm{PV}(x) \simeq y, \mathrm{PP} \doteq(x) \simeq y$ e $\mathrm{PS} \doteq(x) \simeq y$, e. $g$.:

a) Escreveremos $\operatorname{PP} \dot{\wedge}(x) \simeq y$ no lugar de $\exists t_{1}<x\left(\overline{6} * y * t_{1} \approx x \wedge \operatorname{FORM}(y) \wedge\right.$ $\left.\operatorname{FORM}\left(t_{1}\right)\right)$.

Além disso, introduziremos uma operação, por assim dizer, mista:

\subsection{Descrição das contrapartidas formais das instanciações.}

a) Escreveremos $\operatorname{INS}(x, y) \simeq z$ no lugar de $\dot{\exists}(x) \vee \dot{\forall}(x) \wedge(\dot{\exists}(x) \rightarrow \operatorname{PS} \dot{\exists}(x)(\operatorname{PP} \dot{\exists}(x) /$ $y) \simeq z)) \wedge(\dot{\forall}(x) \rightarrow \operatorname{PS} \dot{\forall}(x)(\operatorname{PP} \dot{\forall}(x) / y) \simeq z)) \wedge \mathrm{TF}(y)$.

Iremos supor, ainda, que as contrapartidas formais de " $x$ é uma negação (uma disjunção, uma conjunção, etc.) de complexidade 1" foram adequadamente descritas; tais fórmulas serão denotadas por $\dot{\sim}_{1}(x), \operatorname{SENT} \dot{\sim}_{1}(x), \dot{v}_{1}(x), \operatorname{SENT} \dot{\vee}_{1}(x)$, etc., além de $\operatorname{FORM}_{1}(x)$ e $\operatorname{SENT}_{1}(x)$

\subsection{Fórmulas e sentenças de complexidade 1.}

a) Escreveremos $\dot{\sim}_{1}(x)$ no lugar de $\exists t_{1}<x\left(\operatorname{FORM}_{0}\left(t_{1}\right) \wedge \dot{\sim}\left(t_{1}\right) \approx x\right)$;

b) $\operatorname{SENT} \dot{\sim}_{1}(x)$ no lugar de $\exists t_{1}<x\left(\operatorname{SENT}_{0}\left(t_{1}\right) \wedge \dot{\sim}\left(t_{1}\right) \approx x\right)$.

O mesmo processo é utilizado na descrição de contrapartidas formais para qualquer complexidade dada, e. $g$ :

6.11. Fórmulas e sentenças de complexidade $n+1$.

a) Escreveremos $\dot{\sim}_{n+1}(x)$ no lugar de $\exists t_{n}<x\left(\operatorname{FORM}_{n}\left(t_{n}\right) \wedge \dot{\sim}\left(t_{n}\right) \approx x\right)$;

b) $\operatorname{SENT}_{\sim_{n+1}}(x)$ no lugar de $\exists t_{n}<x\left(\operatorname{SENT}_{n}\left(t_{n}\right) \wedge \dot{\sim}\left(t_{n}\right) \approx x\right)$. 
É possível, e iremos supor que o fizemos, introduzir contrapartidas formais análogas às anteriores, nas quais substituimos a "complexidade lógica" subjacente a elas por noções da hierárquia da aritmética [cf. II §3, pp. 47-49].

Teremos, então, por exemplo:

a) $\operatorname{FORM}_{\Sigma_{0}}(x) \operatorname{e} \operatorname{SENT}_{\Sigma_{0}}(x)$ (de tipo $\Sigma_{0}$ ) tais que $\left|\operatorname{FORM}_{\Sigma_{0}}(x)\right|=\| x$ é uma fórmula $\Sigma_{0}\left\|;\left|\operatorname{SENT}_{\Sigma_{0}}(x)\right|=\right\| x$ é uma sentença $\Sigma_{0} \| ;$

b) $\operatorname{FORM}_{\Pi_{2}}(x)$ e $\operatorname{SENT}_{\Sigma_{5}}(x)$ (de tipo $\Sigma_{0}$ ) tais que $\left|\operatorname{FORM}_{\Pi_{2}}(x)\right|=\| x$ é uma fórmula $\Pi_{2}\left\|;\left|\operatorname{SENT}_{\Sigma_{5}}(x)\right|=\right\| x$ é uma sentença $\Sigma_{5} \|$.

\section{$\S 7$. Resultados relativos às contrapartidas formais}

A maior parte do trabalho realizado até aqui foi a descrição das contrapartidas formais relevantes para nosso estudo. Obviamente, precisaremos de resultados relativos às fórmulas descritas; infelizmente, estabelecer rigorosamente esses resultados para fórmulas, cuja própria leitura é praticamente irrealizável, é extremamente complicado. Nossa esperança é que a descrição das contrapartidas formais e os comentários propostos anteriormente sejam suficientes para vislumbrarmos que podemos em princípio estabelecer os resultados relevantes. Nessa seção, enunciaremos (sem demonstração) alguns dos resultados mais úteis e significativos da "teoria dos termos", entre eles: os lemas da suficiência da decomposição e da unicidade da leitura.

O lema da suficiência da decomposição estabelece que os termos são ou numerais ou somas ou multiplicações ou exponenciações de outros termos.

$$
\begin{aligned}
& \text { Seja } \gamma_{1}(x)={ }_{\text {df. }} \exists y<x(\operatorname{SUC}(y) \approx x \wedge \operatorname{TF}(y)) ; \\
& \gamma_{2}(x)==_{\text {df. }} \exists y<x \exists z<x(\dot{+}(y, z) \approx x \wedge \operatorname{TF}(y) \wedge \operatorname{TF}(z)) ; \\
& \gamma_{3}(x)={ }_{\text {df. }} \exists y<x \exists z<x(:(y, z) \approx x \wedge \operatorname{TF}(y) \wedge \operatorname{TF}(z)) ; \\
& \gamma_{4}(x)=\text { df. } \exists y<x \exists z<x(\operatorname{EXP}(y, z) \approx x \wedge \operatorname{TF}(y) \wedge \operatorname{TF}(z)) . \\
& \text { Seja, finalmente, } \kappa_{1}(x)==_{\text {df. }} \exists y<x(\operatorname{SUC}(y) \approx x \wedge \operatorname{TM}(y)) ;
\end{aligned}
$$




$$
\begin{aligned}
& \kappa_{2}(x)={ }_{\text {df. }} \exists y<x \exists z<x(\dot{+}(y, z) \approx x \wedge \mathrm{TM}(y) \wedge \mathrm{TM}(z)) ; \\
& \kappa_{3}(x)={ }_{\text {df. }} \exists y<x \exists z<x(\dot{0}(y, z) \approx x \wedge \mathrm{TM}(y) \wedge \mathrm{TM}(z)) ; \\
& \kappa_{4}(x)={ }_{\text {df. }} \exists y<x \exists z<x(\operatorname{EXP}(y, z) \approx x \wedge \mathrm{TM}(y) \wedge \mathrm{TM}(z)) .
\end{aligned}
$$

\subsection{Lemas da suficiência da decomposição.}

a) P.A. $\vdash \forall x\left(\operatorname{TF}(x) \rightarrow\left(x=\overline{10} \vee \gamma_{1}(x) \vee \gamma_{2}(x) \vee \gamma_{3}(x) \vee \gamma_{4}(x)\right)\right)$;

b) P.A. $\vdash \forall x\left(\operatorname{TM}(x) \rightarrow\left(\operatorname{VAR}(x) \vee x=\overline{10} \vee \kappa_{1}(x) \vee \kappa_{2}(x) \vee \kappa_{3}(x) \vee \kappa_{4}(x)\right)\right)$.

Os lemas da unicidade da leitura estabelecem que se $\tau$ é um termo de certo tipo (um numeral maior que 0 , por exemplo), então $\tau$ não é um termo de um outro tipo qualquer (não é uma soma, não é uma multiplicação, etc.). Tais lemas podem ser todos estabelecidos em P.A. (na verdade, $\mathrm{I} \Sigma_{0}$ seria suficiente).

\subsection{Lemas da unicidade da leitura (exemplo).}

a) P.A. $\vdash \forall x(\operatorname{TF}(x) \wedge \exists y<x(\operatorname{SUC}(y) \approx x) \rightarrow \sim \exists y \exists z(\dot{+}(y, z) \approx x) \wedge \sim \exists y \exists z(\cdot(y, z) \approx x)$ $\wedge \sim \exists y \exists z(\operatorname{EXP}(y, z) \approx x) \wedge x \neq \overline{10})$.

Uma subfórmula importante de $\operatorname{LTF}(x) \simeq y$ é $\operatorname{SEQ}(s) \wedge \forall w \leqslant \operatorname{COMP}(s)\left(\alpha_{1} \vee \alpha_{2} \vee\right.$ $\left.\alpha_{3} \vee \alpha_{4} \vee \alpha_{5}\right)$ [cf. p. 63] ou, sucintamente, $\operatorname{SEQ}(s) \wedge \forall w \leqslant \operatorname{COMP}(s)\left(\vee \alpha_{1-5}\right)$.

Trata-se de uma contrapartida formal do conceito de "s é uma sequência de valoração de termos fechados". Um lema útil e por si só significativo estabelece que apenas termos fechados têm legendas ou denotação.

\subsection{Lema.}

a) P.A. $\left.\vdash \forall x \forall y\left(\left(\operatorname{SEQ}(x) \wedge \operatorname{COMP}(x) \simeq y \wedge \forall w \leqslant y\left(\vee \alpha_{1-5}\right)\right) \rightarrow \operatorname{TF}\left(\left((x)_{y}\right)_{1}\right)\right)\right)$.

O que, aproveitando algumas propriedades de $\operatorname{SEQ}(x)$ e $\operatorname{COMP}(x) \simeq y$ e segundo nossas convenções semi-formais, pode ser enunciado como:

\subsection{Lema.}

a) P.A. $\vdash \forall x\left(\operatorname{SEQ}(x) \wedge \forall w \leqslant \operatorname{COMP}(x)\left(\vee \alpha_{1-5}\right) \rightarrow \operatorname{TF}\left(\left((x)_{\operatorname{COMP}(x)}\right)_{1}\right)\right)$. 
IV

\section{Definições parciais de verdade e falsidade}

\section{§1. Fórmulas-legenda revisitadas}

Aplicações efetivas e rigorosas dos métodos gödelianos de aritmetização trazem invariavelmente complicações de ordem prática: os números de Gödel são, tipicamente, gigantescos. Muitos autores ensaiam simplificar as coisas, pretendendo preservar o núcleo do argumento; o problema está em como fazê-lo sem comprometer nosso entendimento da própria argumentação: "cobre-se a cabeça, descobrem-se os pés".

$\mathrm{Na}$ teoria das definições parciais de verdade, o problema se torna um pouco pior; falamos de números, numerais, fórmulas, denotação, valorações e das respectivas contrapartidas formais; arriscamo-nos na intrincada teia de suas relações.

Levando isso em conta, procuraremos agora examinar mais detidamente as fórmulas-legenda [cf. III \$5, pp. 62-64] que estão essencialmente relacionadas à aritmetização da relação “evaluation” proposta por Hájek \& Pudlák [1998,1.64-1.66, pp. 53-55]; tais fórmulas estarão na base de nossa teoria das definições parciais de verdade.

Para tanto, seguindo uma prática comum a teóricos de outras especialidades, introduziremos modelos hipotéticos simplificados de uso restrito e temporário. Por exemplo, ao invés de negligenciarmos completamente os detalhes da gödelização da metateoria-afinal dizem: "Deus (ou o Diabo [quem sabe?]) está nos detalhes"-; proporemos gödelizações hipotéticas e temporárias, em oposição à godelização "oficial" proposta anteriormente [cf. III §1, pp. 52-53], com vistas a observar mais de perto a teia de relações ligadas às fórmulas-legenda.

\subsection{Resumo de fatos e retomada da notação.}

a) Para cada expressão $\varepsilon$ das linguagens de base, $g(\varepsilon)$ é o número de Gödel de $\varepsilon$; $g$ é uma função injetora do conjunto expressões das linguagens de base em $\mathbb{N}$ [cf. p. 52]. 
b) $\bar{n}$ é o numeral correspondente ao número natural $n$; $^{-}$é, claramente, uma função bijetora do conjunto dos números naturais no conjuntos dos numerais das linguagens de base [cf. p. 41].

c) Para cada expressão $\varepsilon$ das linguagens de base, $\lceil\varepsilon\rceil$ é $\bar{n}$, onde $n$ é o número de Gödel de $\varepsilon$; \lceil\rceil é a composição entre $g \mathrm{e}^{-}$, ou seja, \lceil\rceil${ }^{-}(g)$; \lceil\rceil é, portanto, uma função injetora do conjunto de expressões no conjunto de numerais das linguagens de base [cf. p. 53].

d) Para cada termo fechado $\tau$ das linguagens de base, $d(\tau)$ é a denotação de $\tau ; d$ é uma função injetora dos termos fechados das linguagens de base em $\mathbb{N}$ [cf. p. 44].

e) Q*, I $\Sigma_{1}$ e P.A. são axiomatizações da aritmética em L.A. [cf. pp. 50-51]. (Todos os teoremas de Q* são teoremas de I $\Sigma_{1}$, todos os teoremas de I $\Sigma_{1}$ são teoremas de P.A.. $E m I \Sigma_{1}$, o esquema de indução está restrito às fórmulas $\Sigma_{1}$.)

1.2. Propriedades básicas de $\operatorname{LTF}^{\Sigma}(x) \simeq y$ e $\operatorname{LTF}^{\Pi}(x) \simeq y$ [cf. pp. 64-64].

a) $\operatorname{LTF}^{\Sigma}(x) \simeq y$ é $\Sigma_{1}$ e as únicas variáveis livres de $\operatorname{LTF}^{\Sigma}(x) \simeq y$ são $x$ e $y$.

b) $\operatorname{LTF}^{\Pi}(x) \simeq y$ é $\Pi_{1}$ e as únicas variáveis livres de $\operatorname{LTF}^{\Pi}(x) \simeq y$ são $x$ e $y$.

c) $\mathrm{I}_{1} \vdash \forall x \forall y\left(\operatorname{LTF}^{\Sigma}(x) \simeq y \leftrightarrow \operatorname{LTF}^{\Pi}(x) \simeq y\right)$.

d) $\mathrm{I}_{1} \vdash \forall x \forall y \forall z\left(\left(\operatorname{LTF}^{\Sigma}(x) \simeq y \wedge \operatorname{LTF}^{\Sigma}(x) \simeq z\right) \rightarrow y=z\right)$.

e) $\operatorname{I} \Sigma_{1} \vdash \forall x\left(\operatorname{TF}(x) \rightarrow \exists y\left(\operatorname{LTF}^{\Sigma}(x) \simeq y\right)\right)$.

f) Se $\tau$ é um termo fechado e $d(\tau)=n$, então $I_{1}+\operatorname{LTF}^{\Sigma}(\lceil\tau\rceil) \simeq \bar{n}$.

g) Se $\operatorname{I}_{1}+\operatorname{LTF}^{\Sigma}(\bar{m}) \simeq \bar{n}$, então existe um termo fechado $\tau$ tal que $g^{-1}(m)=\tau$ e $d(\tau)=n$.

Ou seja, $\operatorname{LTF}^{\Sigma}(x) \simeq y$ e $\operatorname{LTF}^{\Pi}(x) \simeq y$ são contrapartidas formais da denotação de termos fechados das linguagens de base por números naturais.

Nossas "propriedades básicas" não são, na maioria dos casos, absolutamente simples de serem estabelecidas. Um exame mais próximo, por exemplo, das condições 
necessárias à demonstração de 1.2.e) conduz à formulação de uma "teoria dos termos" que, apesar de certo grau de trivialidade, é razoavelmente difícil de ser estabelecida rigorosamente; devemos, entre outras coisas, nela demonstrar as contrapartidas formais dos "lemas da unicidade da leitura" [cf. p. 71].

1.3. Alguns exemplos do comportamento de $\operatorname{LTF}^{\Sigma}(x) \simeq y$. Apresentaremos uma gödelização (da forma já abreviada) de $L_{\text {P.A. }}$ de caráter temporário e parcial cuja finalidade é proporcionar uma melhor intuição do funcionamento das fórmulas-legenda. Suponhamos, por exemplo, que os números de Gödel de expressões de $L_{P . A}$ são o resultado da concatenação na base 10 segundo a seguinte atribuição de números aos símbolos de $L_{\text {P.A. }}$ :

$$
\begin{array}{rlrl}
\overline{0} & \mapsto 1 & + & \mapsto 2 \\
\overline{1} & \mapsto 10 & \cdot & \mapsto 3 \\
\vdots & & \mapsto 4 \\
\bar{n} & \mapsto 10^{n} & & \mapsto 5
\end{array}
$$

Segue-se, então, que:
a) $g(\overline{1}+\overline{2})=102100$;
b) $d(\overline{1}+\overline{2})=3$;
c) Assim, por 1.1, $\lceil\overline{1}+\overline{2}\rceil=\overline{102} \overline{100}$;
d) Por 1.2.f), temos que $\mathrm{I}_{1}+\operatorname{LTF}^{\Sigma}(\overline{102100}) \simeq \overline{3}$, ou seja, $\operatorname{I\Sigma }_{1}+\operatorname{LTF}^{\Sigma}(\lceil\overline{1}+\overline{2}\rceil) \simeq \overline{3}$.

Para um exemplo mais complexo, temos:

a) $g((\overline{2}+\overline{2}) \cdot \overline{2})=4100210053100$;

b) $d((\overline{2}+\overline{2}) \cdot \overline{2})=8$;

c) Por 1.1, $\lceil(\overline{2}+\overline{2}) \cdot \overline{2}\rceil=\overline{4100210053100}$;

d) E, por 1.2.f), temos que $\Sigma_{1}+\operatorname{LTF}^{\Sigma}(\lceil(\overline{2}+\overline{2}) \cdot \overline{2}\rceil) \simeq \overline{8}$. 
Os fatos abaixo são válidos para $\operatorname{LTF}^{\Sigma}(x) \simeq y$ e $\operatorname{LTF}^{\Pi}(x) \simeq y$, expressaremos tal situação apagando os índices superiores das fórmulas-legenda.

\subsection{Alguns fatos básicos sobre $\operatorname{LTF}(x) \simeq y$.}

a) Para qualquer $n \in \mathbb{N}, \operatorname{I} \Sigma_{1} \vdash \operatorname{LTF}(\lceil\bar{n}\rceil) \simeq \bar{n}$, ou seja, $\left.\operatorname{I} \Sigma_{1} \vdash \operatorname{LTF}(\overline{g(\bar{n}})\right) \simeq \bar{n}$; por exemplo, $\operatorname{I} \Sigma_{1} \vdash \operatorname{LTF}(\overline{10}) \simeq \overline{0}, \operatorname{I} \Sigma_{1} \vdash \operatorname{LTF}(\overline{110} \overline{10}) \simeq \overline{1}$ e $\operatorname{I} \Sigma_{1} \vdash \operatorname{LTF}(\overline{110110} \overline{10}) \simeq \overline{2}$ (nesse exemplo, usamos nossa godelização oficial). De fato, temos que, para qualquer termo fechado $\tau, \operatorname{I} \Sigma_{1}+\operatorname{LTF}(\lceil\tau\rceil) \simeq \tau$;

b) Para quaisquer $m$, $n$ e $a \in \mathbb{N}$ tal que $m+n=a, \operatorname{I} \Sigma_{1} \vdash \operatorname{LTF}(\lceil\bar{m}+\bar{n}\rceil) \simeq \bar{a}$; notemos que o símbolo de adição em ' $m+n$ ' é parte da linguagem da aritmética informal, enquanto em ' $\bar{m}+\bar{n}$ ' é uma constante de $\mathrm{L}_{\text {P.A. }}$ (mutatis mutandis o comentário se aplica aos próximos resultados);

c) Para quaisquer $m, n$ e $a \in \mathbb{N}$ tal que $m \cdot n=a, \operatorname{I} \Sigma_{1}+\operatorname{LTF}(\lceil\bar{m} \cdot \bar{n}\rceil) \simeq \bar{a}$;

d) Para quaisquer $m, n$ e $a \in \mathbb{N}$ tal que $m^{n}=a, \operatorname{I} \Sigma_{1} \vdash \operatorname{LTF}\left(\left\lceil f_{3}^{2} \bar{m} \bar{n}\right\rceil\right) \simeq \bar{a}$;

e) Ou, mais genericamente, dado que I $\Sigma_{1}$ é $\Sigma_{0}$-completo, temos que, para quaisquer termos fechados $\tau_{1}$ e $\tau_{2}$ tais que $d\left(\tau_{1}\right)=d\left(\tau_{2}\right), \operatorname{I} \Sigma_{1} \vdash \operatorname{LTF}\left(\left\lceil\tau_{1}\right\rceil\right) \simeq \overline{d\left(\tau_{2}\right)}$ e, portanto, mais especificamente, que $\operatorname{I} \Sigma_{1} \vdash \operatorname{LTF}\left(\left\lceil\tau_{1}\right\rceil\right) \simeq \overline{d\left(\tau_{1}\right)}$;

f) Obviamente, é falso que se $\mathbb{N} \vDash \operatorname{LTF}(\lceil\tau\rceil) \simeq \bar{n}$, então $\mathbb{N} \vDash\lceil\tau\rceil=\bar{n}$; é (menos óbvio, mas) falso que se $\mathbb{N} \vDash \operatorname{LTF}(\lceil\tau\rceil) \simeq \bar{n}$, então $\mathbb{N} \vDash \bar{n} \leqslant\lceil\tau\rceil$.

Dados 4.e)-f), temos duas questões interessantes:

a) Existe algum $\tau$ tal que $\mathbb{N} \vDash \operatorname{LTF}(\tau) \simeq \tau$ ?

b) $E$, supondo que a resposta seja positiva, essa espécie de teorema do ponto-fixo dependeria dos pormenores de uma gödelização específica ? 


\section{§2. Definição de verdade para sentenças atômicas}

A definição de verdade para fórmulas atômicas demanda algumas fórmulas auxiliares cujas propriedades básicas serão resumidas abaixo (as variáveis livres das fórmulas em questão são aquelas indicadas explicitamente).

2.1. Algumas propriedades básicas de $\doteq(x), \operatorname{SENT} \doteq(x), \mathrm{PP} \doteq(x) \simeq y$, $\mathrm{PS} \doteq(x) \simeq y$ [cf. pp. 65-66].

a) $\doteq(x), \mathrm{SENT} \doteq(x), \mathrm{PP} \doteq(x) \simeq y$ e $\mathrm{PS} \doteq(x) \simeq y$ são $\Sigma_{0}$.

b) $\mathrm{I} \Sigma_{1} \vdash \forall x \forall y \forall z((\mathrm{PP} \doteq(x) \simeq y \wedge \mathrm{PP} \dot{=}(x) \simeq z) \rightarrow y=z)$.

c) $\mathrm{I} \Sigma_{1} \vdash \forall x \forall y \forall z((\mathrm{PS} \dot{\doteq}(x) \simeq y \wedge \mathrm{PS} \dot{\doteq}(x) \simeq z) \rightarrow y=z)$.

d) $\mathrm{I} \Sigma_{1} \vdash \forall x(\dot{=}(x) \rightarrow \exists y(\mathrm{PP} \dot{=}(x) \simeq y))$.

e) $\mathrm{I} \Sigma_{1} \vdash \forall x(\doteq(x) \rightarrow \exists y(\mathrm{PS} \doteq(x) \simeq y))$.

f) $\mathrm{I} \Sigma_{1} \vdash \forall x(\operatorname{SENT} \doteq(x) \rightarrow \dot{\doteq}(x))$.

g) Se $\tau_{1}$ e $\tau_{2}$ são termos de $L_{\text {P.A. }}$, então $I \Sigma_{1} \vdash \doteq\left(\left\lceil\tau_{1}=\tau_{2}\right\rceil\right)$.

h) Se I $\Sigma_{1} \vdash \doteq(\bar{n})$, então existem termos $\tau_{1}$ e $\tau_{2}$ de $L_{\text {P.A. }}$ tais que $g^{-1}(n)$ é $\tau_{1}=\tau_{2}$.

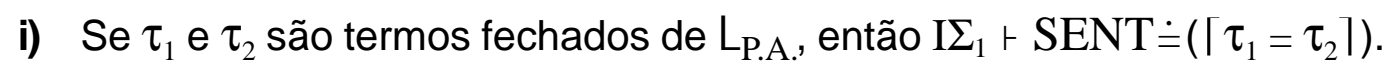

j) Se I $\Sigma_{1}+\operatorname{SENT} \doteq(\bar{n})$, então existem termos fechados $\tau_{1}$ e $\tau_{2}$ de $L_{\text {P.A. }}$ tais que $g^{-1}(n)$ é $\tau_{1}=\tau_{2}$.

l) Se $\tau_{1}$ e $\tau_{2}$ são termos de $L_{P . A}$, então $\mathrm{I} \Sigma_{1} \vdash \mathrm{PP} \doteq\left(\left\lceil\tau_{1}=\tau_{2}\right\rceil\right) \simeq\left\lceil\tau_{1}\right\rceil$.

m) Se I $\Sigma_{1} \vdash \mathrm{PP} \doteq(\bar{m}) \simeq \bar{n}$, então existem termos $\tau_{1}$ e $\tau_{2}$ de $L_{\text {P.A. }}$ tais que $g^{-1}(m)$ é $\tau_{1}=\tau_{2}$ e $g^{-1}(n)$ é $\tau_{1}$.

n) Se $\tau_{1}$ e $\tau_{2}$ são termos de $L_{\text {P.A. }}$, então $\mathrm{I} \Sigma_{1} \vdash \mathrm{PS} \doteq\left(\left\lceil\tau_{1}=\tau_{2}\right\rceil\right) \simeq\left\lceil\tau_{2}\right\rceil$.

o) Se I $\Sigma_{1} \vdash \mathrm{PS} \doteq(\bar{m}) \simeq \bar{n}$, então existem termos $\tau_{1}$ e $\tau_{2}$ de $L_{\text {P.A. }}$ tais que $g^{-1}(m)$ é $\tau_{1}=\tau_{2}$ e $g^{-1}(n)$ é $\tau_{2}$.

Ou seja $, \doteq(x), \mathrm{SENT} \doteq(x), \mathrm{PP} \doteq(x) \simeq y$ e $\mathrm{PS} \doteq(x) \simeq y$ são contrapartidas formais, respectivamente, de " $x$ é uma equação", " $x$ é uma equação sem variáveis livres", 
" $y$ é a projeção do primeiro termo da equação $x$ " e " $y$ é a projeção do segundo termo da equação $x$ " [cf. pp. 64-65].

2.2. Convenção. Se $\varphi\left(x_{1}, \ldots, x_{n}\right)$ é tal que

a) $\mathrm{T} \vdash \forall x_{1} \ldots \forall x_{n} \forall y\left(\varphi\left(x_{1}, \ldots, x_{n}\right) \wedge \varphi\left(x_{1}, \ldots, x_{n-1}, y\right) \supset x_{n}=y\right) \mathrm{e}$

b) $\mathrm{T} \vdash \forall x_{1} \ldots \forall x_{n-1}\left(\psi\left(x_{1}, \ldots, x_{n-1}\right) \rightarrow \exists y\left(\varphi\left(x_{1}, \ldots, x_{n-1}, y\right)\right)\right.$, então $\varphi\left(x_{1}, \ldots, x_{n}\right)$ é $\psi$-funcional em $\mathrm{T}$.

2.3. Algumas propriedades básicas de $\dot{\leqslant}(x), \operatorname{SENT} \dot{\leqslant}(x), \operatorname{PP} \dot{\leqslant}(x) \simeq y$ e $\mathrm{PS} \dot{\leqslant}(x) \simeq y$ [cf. pp. 65-66].

a) $\dot{\leqslant}(x), \operatorname{SENT} \dot{\leqslant}(x), \operatorname{PP} \dot{\leqslant}(x) \simeq y$ e $\mathrm{PS} \dot{\leqslant}(x) \simeq y$ são $\Sigma_{0}$.

b) $\mathrm{PP} \dot{\leqslant}(x) \simeq y$ e $\mathrm{PS} \dot{\leqslant}(x) \simeq y$ são $\dot{\leqslant}$-funcionais em $\mathrm{I} \Sigma_{1}$.

c) $\mathrm{I} \Sigma_{1} \vdash \forall x(\operatorname{SENT} \dot{\leqslant}(x) \rightarrow \dot{\leqslant}(x))$.

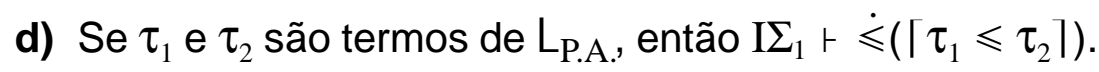

e) Se I $\Sigma_{1} \vdash \dot{\xi}(\bar{n})$, então existem termos $\tau_{1}$ e $\tau_{2}$ de $L_{\text {P.A. }}$ tais que $g^{-1}(n)$ é $\tau_{1} \leqslant \tau_{2}$.

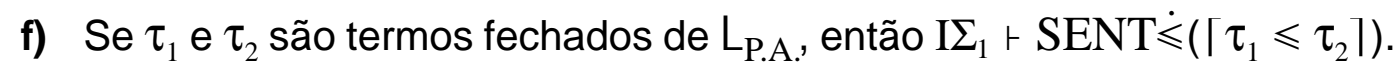

g) Se I $\Sigma_{1}+\operatorname{SENT} \leqslant(\bar{n})$, então existem termos fechados $\tau_{1}$ e $\tau_{2}$ de $L_{\text {P.A. }}$ tais que $g^{-1}(n)$ é $\tau_{1} \leqslant \tau_{2}$.

h) Se $\tau_{1}$ e $\tau_{2}$ são termos de $L_{\text {P.A. }}$, então $\mathrm{I} \Sigma_{1} \vdash \mathrm{PP} \dot{\leqslant}\left(\left\lceil\tau_{1} \leqslant \tau_{2}\right\rceil\right) \simeq\left\lceil\tau_{1}\right\rceil$.

i) Se I $\Sigma_{1} \vdash \mathrm{PP} \dot{\leqslant}(\bar{m}) \simeq \bar{n}$, então existem termos $\tau_{1}$ e $\tau_{2}$ de $L_{\text {P.A. }}$ tais que $g^{-1}(m)$ é $\tau_{1} \leqslant \tau_{2}$ e $g^{-1}(n)$ é $\tau_{1}$.

j) Se $\tau_{1}$ e $\tau_{2}$ são termos de $L_{\text {P.A. }}$, então $\mathrm{I} \Sigma_{1} \vdash \mathrm{PS} \dot{\leqslant}\left(\left\lceil\tau_{1} \leqslant \tau_{2}\right\rceil\right) \simeq\left\lceil\tau_{2}\right\rceil$.

I) Se I $\Sigma_{1}+\mathrm{PS} \dot{\leqslant}(\bar{m}) \simeq \bar{n}$, então existem termos $\tau_{1}$ e $\tau_{2}$ de $L_{\text {P.A. }}$ tais que $g^{-1}(m)$ é $\tau_{1} \leqslant \tau_{2}$ e $g^{-1}(n)$ é $\tau_{2}$.

As propriedades 2.3 deixam claro que $\dot{\leqslant}(x)$ é uma contrapartida formal de " $x$ é uma inequação"; $\operatorname{SENT} \leqslant(x)$ é uma contrapartida de " $x$ é uma inequação sem variáveis"; 
$\mathrm{PP} \dot{\leqslant}(x) \simeq y$ é uma contrapartida de " $y$ é a projeção do primeiro termo da inequação $x$ "; $\mathrm{PS} \dot{\leqslant}(x) \simeq y$, de " $y$ é a projeção do segundo termo da inequação $x$ " [cf. pp. 65-66].

Poderemos, então, introduzir às contrapartidas formais $\Sigma_{1}$ e $\Pi_{1}$ de " $x$ é uma equação verdadeira em $\mathbb{N}$ " e " $x$ é uma inequação verdadeira em $\mathbb{N}$ ". A ideia subjacente à definição de "verdade em $\mathbb{N}$ para equações" será, grosso modo, a seguinte: uma equação $\tau_{1}=\tau_{2}$ é verdadeira se existe uma sequência, cujo primeiro elemento é uma valoração de $\tau_{1}$ e cujo segundo elemento é uma valoração de $\tau_{2}$, tal que as valorações estabelecem uma mesma denotação para os termos $\tau_{1}$ e $\tau_{2}$.

\subsection{Descrição semi-formal $\Sigma_{1}$ da verdade em $\mathbb{N}$ para equações.}

a) Escreveremos $\mathrm{V}_{=}^{\Sigma}(x)$ no lugar de

$\exists s\left(\mathrm{SEQ}(s) \wedge \operatorname{COMP}(s)=\overline{2} \wedge \operatorname{SEQ}\left((s)_{1}\right) \wedge \mathrm{SEQ}\left((s)_{2}\right) \wedge \forall w \leqslant \operatorname{COMP}\left((s)_{1}\right)\left(\alpha_{1-5}\right)\right.$ $\wedge \forall w \leqslant \operatorname{COMP}\left((s)_{2}\right)\left(\alpha_{1-5}\right) \wedge \mathrm{PP} \dot{\doteq}(x)=\left(\left((s)_{1}\right)_{\mathrm{COMP}\left((s)_{1}\right)}\right)_{1} \wedge \mathrm{PS} \dot{\doteq}(x)=\left(\left((s)_{2}\right)_{\mathrm{COMP}\left((s)_{2}\right)_{1}} \wedge\right.$

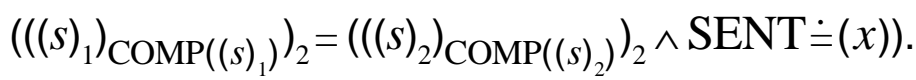

Onde $\alpha_{1-5}$ é $\left(\alpha_{1} \vee \alpha_{2} \vee \alpha_{3} \vee \alpha_{4} \vee \alpha_{5}\right)$ e as subfórmulas $\alpha_{1}, \alpha_{2}, \alpha_{3}, \alpha_{4}$ e $\alpha_{5}$ [cf. pp. 61-62] são, respectivamente:

$$
\begin{aligned}
& \left.(s)_{w}=\langle\overline{10}, \overline{0}\rangle \text { (ou seja, de }(s)_{w}=\langle\lceil\overline{0}\rceil, \overline{0}\rangle\right) ; \\
& \exists v<w\left((s)_{w}=\left\langle\operatorname{SUC}\left(\left((s)_{v}\right)_{1}\right), f_{1}^{1}\left((s)_{v_{2}}\right)_{2}\right\rangle\right) ; \\
& \exists v_{1}<w \exists v_{2}<w\left((s)_{w}=\left\langle\dot{+}\left(\left((s)_{v_{1}}\right)_{1},\left((s)_{v_{2}}\right)_{1}\right),\left((s)_{v_{1}}\right)_{2}+\left((s)_{v_{2}}\right)_{2}\right\rangle\right) ; \\
& \exists v_{1}<w \exists v_{2}<w\left((s)_{w}=\left\langle\dot{(}\left(\left((s)_{v_{1}}\right)_{1},\left((s)_{v_{2}}\right)_{1}\right),\left((s)_{v_{1}}\right)_{2} \cdot\left((s)_{v_{2}}\right)_{2}\right\rangle\right) ; \\
& \exists v_{1}<w \exists v_{2}<w\left((s)_{w}=\left\langle\operatorname{EXP}\left(\left((s)_{v_{1}}\right)_{1},\left((s)_{v_{2}}\right)_{1}\right), f_{3}^{2}\left((s)_{v_{1}}\right)_{2}\left((s)_{v_{2}}\right)_{2}\right\rangle\right) .
\end{aligned}
$$

Menos rigorosamente, teríamos:

\subsection{Descrição semi-formal $\Sigma_{1}$ da verdade em $\mathbb{N}$ para equações.}

a) Escreveremos $\mathrm{V}_{=}^{\Sigma}(x)$ no lugar de

$\mathrm{SENT} \doteq(x) \wedge \operatorname{LTF}^{\Sigma}(\mathrm{PP} \doteq(x))=\operatorname{LTF}^{\Sigma}(\mathrm{PS} \doteq(x))$. 
Obviamente, $\left|\mathrm{V}_{=}^{\Sigma}(x)\right| \neq|\mathrm{SENT} \doteq(x) \wedge \mathrm{PP} \doteq(x)=\mathrm{PS} \doteq(x)|$, em outras palavras, o conjunto das equações verdadeiras em $\mathbb{N}$ é diferente do conjunto dos números de Gödel de equações cujos dois termos, enquanto termos, são iguais; muito embora $|\mathrm{SENT} \doteq(x) \wedge \mathrm{PP} \doteq(x)=\mathrm{PS} \doteq(x)|$ seja, obviamente, um subconjunto de $\left|\mathrm{V}_{=}^{\Sigma}(x)\right|$.

Temos, por exemplo, que $\mathbb{N} \vDash V_{=}^{\Sigma}(\lceil\overline{1}+\overline{2}=\overline{3}\rceil)$ e, uma vez que $\mathbb{N} \vDash\lceil\overline{1}+\overline{2}\rceil \neq\lceil\overline{3}\rceil$, que $\mathbb{N} \vDash \mathrm{PP} \doteq(\lceil\overline{1}+\overline{2}=\overline{3}\rceil) \neq \mathrm{PS} \doteq(\lceil\overline{1}+\overline{2}=\overline{3}\rceil)$.

Como havíamos explicado anteriormente [cf. p. 61], nossa notação semi-formal pressupõe as condições de comutação dos gráficos de funções parciais, tomando, por essa razão, as abreviações das fórmulas correspondentes como se fossem símbolos funcionais.

Não obstante, um pouco de paciência permitiria uma descrição mais perspícua (e de leitura mais difícil) de $\mathrm{V}_{=}^{\Sigma}(x)$.

\subsection{Descrição semi-formal $\Sigma_{1}$ alternativa da verdade em $\mathbb{N}$ para equações.}

a) Escreveremos $\mathrm{V}_{=}^{\Sigma}(x)$ no lugar de $\exists s\left(\operatorname{SEQ}(s) \wedge \operatorname{COMP}(s) \simeq \overline{2} \wedge \exists s_{1} \leqslant s \exists s_{2} \leqslant s\right.$ $\exists c_{1} \leqslant s_{1} \exists c_{2} \leqslant s_{2} \exists s_{10} \leqslant s_{1} \exists s_{20} \leqslant s_{2} \exists t_{1} \leqslant s_{10} \exists t_{2} \leqslant s_{20} \exists v \leqslant s_{10}\left(\operatorname{SEQ}\left(s_{1}\right) \wedge \mathrm{SEQ}\left(s_{2}\right) \wedge(s)_{1} \simeq s_{1}\right.$ $\wedge(s)_{2} \simeq s_{2} \wedge \operatorname{COMP}\left(s_{1}\right) \simeq c_{1} \wedge \operatorname{COMP}\left(s_{2}\right) \simeq c_{2} \wedge \forall w \leqslant c_{1}\left(\alpha_{1-5}\right) \wedge \forall w \leqslant c_{2}\left(\alpha_{1-5}\right) \wedge$ $\mathrm{PP} \doteq(x) \simeq t_{1} \wedge\left(s_{1}\right)_{c_{1}} \simeq s_{10} \wedge\left(s_{10}\right)_{1} \simeq t_{1} \wedge \mathrm{PS} \dot{\doteq}(x) \simeq t_{2} \wedge\left(s_{2}\right)_{c_{2}} \simeq s_{20} \wedge\left(s_{20}\right)_{1} \simeq t_{2} \wedge\left(s_{10}\right)_{2} \simeq v \wedge$ $\left.\left(s_{20}\right)_{2} \simeq v \wedge \operatorname{SENT} \doteq(x)\right)$.

Analogamente à modificação apresentada no caso das fórmulas-legenda $\Sigma_{1} \mathrm{e}$ $\Pi_{1}$ [cf. p. 65], temos:

\subsection{Descrição semi-formal $\Pi_{1}$ da verdade em $\mathbb{N}$ para equações.}

a) Escreveremos $\mathrm{V}_{=}^{\Pi}(x)$ no lugar de $\forall s\left(\left(\left(\beta_{1} \wedge \beta_{2} \wedge \beta_{3} \wedge \beta_{4} \wedge \beta_{5} \wedge \beta_{6} \wedge \beta_{7} \wedge \beta_{8}\right) \rightarrow \beta_{9}\right) \wedge \beta_{10}\right)$.

b) Onde $\beta_{1}$ está no lugar de $\operatorname{SEQ}(s)$;

c) $\beta_{2}$ no lugar de $\operatorname{COMP}(s)=\overline{2}$; 
d) $\beta_{3}$ no lugar de $\operatorname{SEQ}\left((s)_{1}\right)$;

e) $\beta_{4}$ no lugar de $\operatorname{SEQ}\left((s)_{2}\right)$;

f) $\beta_{5}$ no lugar de $\forall w \leqslant \operatorname{COMP}\left((s)_{1}\right)\left(\alpha_{1} \vee \alpha_{2} \vee \alpha_{3} \vee \alpha_{4} \vee \alpha_{5}\right)$;

g) $\beta_{6}$ no lugar de $\forall w \leqslant \operatorname{COMP}\left((s)_{2}\right)\left(\alpha_{1} \vee \alpha_{2} \vee \alpha_{3} \vee \alpha_{4} \vee \alpha_{5}\right)$;

h) $\beta_{7}$ no lugar de $\mathrm{PP} \doteq(x)=\left(\left((s)_{1}\right)_{\mathrm{COMP}\left((s)_{1}\right)}\right)_{1}$;

i) $\beta_{8}$ no lugar de $\mathrm{PS} \doteq(x)=\left(\left((s)_{2}\right)_{\mathrm{COMP}\left((s)_{2}\right)}\right)_{1}$;

j) $\beta_{9}$ no lugarde $\left(\left((s)_{1}\right)_{\operatorname{COMP}\left((s)_{1}\right)}\right)_{2}=\left(\left((s)_{2}\right)_{\operatorname{COMP}\left((s)_{2}\right)}\right)_{2}$;

I) $\beta_{10}$ no lugar de $\operatorname{SENT} \doteq(x)$.

Menos rigorosa e mais simplesmente, teríamos:

2.8. Descrição semi-formal $\Pi_{1}$ da verdade em $\mathbb{N}$ para equações.

a) Escreveremos $\mathrm{V}_{=}^{\Pi}(x)$ no lugar de

$\mathrm{SENT} \doteq(x) \wedge \operatorname{LTF}^{\Pi}(\mathrm{PP} \doteq(x))=\operatorname{LTF}^{\Pi}(\mathrm{PS} \doteq(x))$.

Modificações óbvias das descrições anteriores estabeleceriam as contrapartidas formais $\Sigma_{1}$ e $\Pi_{1}$ de "a inequação $x$ é verdadeira em $\mathbb{N}$ ". Na descrição mais simples, temos:

\subsection{Descrição semi-formal da verdade em $\mathbb{N}$ para inequações.}

a) Escreveremos $\mathrm{V}_{\leqslant}^{\Sigma}(x)$ no lugar de

$\mathrm{SENT} \dot{\xi}(x) \wedge \operatorname{LTF}^{\Sigma}(\mathrm{PP} \doteq(x)) \leqslant \operatorname{LTF}^{\Sigma}(\mathrm{PS} \doteq(x)) ;$

b) $\mathrm{V} \leqslant(x)$ no lugar de $\operatorname{SENT} \dot{\leqslant}(x) \wedge \operatorname{LTF}^{\Pi}(\mathrm{PP} \doteq(x)) \leqslant \operatorname{LTF}^{\Pi}(\mathrm{PS} \doteq(x))$.

\subsection{Alguns exemplos do comportamento das fórmulas.}

Uma vez que a compreensão das definições parciais de verdade para equações e inequações é de extrema importância (essas fórmulas serão a base de todas as definições parciais subsequentes), vale a pena apresentarmos novamente uma gödelização de $L_{P . A}$ de caráter temporário, visando uma melhor intuição do funcionamento dessas. 
Suponhamos que os números de Gödel de expressões de $L_{\text {P.A. são, novamente, }}$ resultado da concatenação na base 10, mas, agora, segundo a atribuição de números aos símbolos de $L_{P . A}$ dada abaixo:

$$
\begin{aligned}
& \overline{0} \mapsto 1 \\
& +\mapsto 2 \\
& \overline{1} \mapsto 10 \\
& \text { - } \mapsto 3 \\
& \vdots \\
& =\mapsto 4 \\
& \bar{n} \mapsto 10^{n} \\
& \leqslant \mapsto 5
\end{aligned}
$$

Segue-se, então, que:

a) $g(\overline{1}+\overline{1}=\overline{2})=102104100$;

b) $d(\overline{1}+\overline{1})=2$ e $d(\overline{2})=2$;

c) Por 1.1, $\lceil\overline{1}+\overline{1}=\overline{2}\rceil=\overline{102104100}$;

d) Por 2.1.g), I $I \Sigma_{1} \vdash \doteq(\overline{102104100})$, ou seja, I $\mathrm{I} \Sigma_{1} \vdash \doteq(\lceil\overline{1}+\overline{1}=\overline{2}\rceil)$;

e) Por 2.1.I), $\mathrm{I} \Sigma_{1}+\mathrm{PP} \doteq(\overline{102104100}) \simeq \overline{10210}$ ou $\mathrm{I} \Sigma_{1}+\mathrm{PP} \doteq(\lceil\overline{1}+\overline{1}=\overline{2}\rceil) \simeq\lceil\overline{1}+\overline{1}\rceil$;

f) Por 2.1.n), I $\Sigma_{1}+\mathrm{PS} \doteq(\lceil\overline{1}+\overline{1}=\overline{2}\rceil) \simeq\lceil\overline{2}\rceil$;

g) Por 1.2.f), $\operatorname{I} \Sigma_{1}+\operatorname{LTF}(\lceil\overline{2}\rceil) \simeq \overline{2}$;

h) Por 1.2.f), $\operatorname{I} \Sigma_{1}+\operatorname{LTF}(\lceil\overline{1}+\overline{1}\rceil) \simeq \overline{2}$;

i) Por d)-h) e 2.4, I $\Sigma_{1}+V_{=}(\overline{102104100})$, ou seja, $I \Sigma_{1}+V_{=}(\lceil\overline{1}+\overline{1}=\overline{2}\rceil)$.

E também que:

a) $g(\overline{1} \cdot \overline{1} \leqslant \overline{2})=103105100$;

b) $d(\overline{1} \cdot \overline{1})=2$ e $d(\overline{2})=2$;

c) Por 2.2.d), I $\Sigma_{1} \vdash \dot{\leqslant}(\lceil\overline{1} \cdot \overline{1} \leqslant \overline{2}\rceil)$, ou seja, $\mathrm{I} \Sigma_{1} \vdash \dot{\leqslant}(\overline{103105100})$;

d) Por 2.2.h), $\mathrm{I} \Sigma_{1}+\mathrm{PP} \dot{\leqslant}(\lceil\overline{1} \cdot \overline{1} \leqslant \overline{2}\rceil) \simeq\lceil\overline{1} \cdot \overline{1}\rceil$;

e) Por 2.2.j), I $\Sigma_{1}+\mathrm{PS} \dot{\leqslant}(\lceil\overline{1} \cdot \overline{1} \leqslant \overline{2}\rceil) \simeq\lceil\overline{2}\rceil$;

f) Por 1.2.f), $\operatorname{I\Sigma } \Sigma_{1}+\operatorname{LTF}(\lceil\overline{2}\rceil) \simeq \overline{2}$;

g) Por 1.2.f), $\operatorname{I} \Sigma_{1}+\operatorname{LTF}(\lceil\overline{1} \cdot \overline{1}\rceil) \simeq \overline{2}$; 
h) $\mathrm{I} \Sigma_{1}+\overline{2} \leqslant \overline{2}$;

i) Por c)-h) e 2.9, $I \Sigma_{1}+V_{\leqslant}(\lceil\overline{1} \cdot \overline{1} \leqslant \overline{2}\rceil)$.

2.11. Fórmulas auxiliares. Descreveremos as contrapartidas formais de " $x$ é uma sentença atômica verdadeira em $\mathbb{N}$ ", ajudados pelas seguintes abreviações mais adequadamente estruturadas:

a) Escreveremos $\exists s\left(\Phi_{=}^{\Sigma}(s, x)\right)$ no lugar de $\mathrm{V}_{=}^{\Sigma}(x)$;

b) $\exists s(\varpi \stackrel{\Sigma}{\leqslant}(s, x))$ no lugar de $\mathrm{V}_{\leqslant}^{\Sigma}(x)$;

c) $\forall s\left(\varpi_{=}^{\Pi}(s, x)\right)$ no lugar de $\mathrm{V}_{=}^{\Pi}(x)$;

d) $\forall s\left(\varpi_{\leqslant}^{\Pi}(s, x)\right)$ no lugar de $\mathrm{V}_{\leqslant}^{\Sigma}(x)$.

2.12. Descrição semi-formal da verdade em $\mathbb{N}$ para sentenças atômicas.

a) Escreveremos $\mathrm{V}_{0}^{\Sigma}(x)$ no lugar de $\exists s\left(\Phi_{=}^{\Sigma}(s, x) \vee \Phi_{\leqslant}^{\Sigma}(s, x)\right)$.

b) $\mathrm{V}_{0}^{\Pi}(x)$ no lugar de $\forall s\left(\left(\operatorname{SENT} \dot{=}(x) \rightarrow \varpi_{=}^{\Pi}(s, x)\right) \wedge\left(\operatorname{SENT} \dot{\xi}(x) \rightarrow \varpi_{\leqslant}^{\Pi}(s, x)\right) \wedge\right.$ $(\operatorname{SENT} \doteq(x) \vee \operatorname{SENT} \dot{\leqslant}(x)))$.

2.13. Propriedades básicas de $\mathrm{V}_{0}^{\Sigma}(x)$ e $\mathrm{V}_{0}^{\Pi}(x)$.

a) $\mathrm{V}_{0}^{\Sigma}(x)$ é $\Sigma_{1}$ e a única variável livre de $\mathrm{V}_{0}^{\Sigma}(x)$ é $x$.

b) $\mathrm{V}_{0}^{\Pi}(x)$ é $\Pi_{1}$ e a única variável livre de $\mathrm{V}_{0}^{\Pi}(x)$ é $x$.

c) $\mathrm{I} \Sigma_{1} \vdash \forall x\left(\mathrm{~V}_{0}^{\Sigma}(x) \leftrightarrow \mathrm{V}_{0}^{\Pi}(x)\right)$ [cf. Hájek \& Pudlák, 1998, p. 58].

d) Se $\alpha$ é uma sentença atômica de $L_{\text {P.A. }}$ tal que $\mathbb{N} \vDash \alpha$, então $I \Sigma_{1}+V_{0}(\lceil\alpha\rceil)$.

e) Se $\mathrm{I} \Sigma_{1} \vdash \mathrm{V}_{0}(\bar{n})$, então $g^{-1}(n)$ é uma sentença atômica de $L_{\text {P.A. }}$ e $\mathbb{N} \vDash g^{-1}(n)$.

Ou seja, $\mathrm{V}_{0}^{\Sigma}(x)$ e $\mathrm{V}_{0}^{\Pi}(x)$ são contrapartidas formais de " $x$ é uma sentença atômica verdadeira em $\mathbb{N}$ ".

Notemos que, por meio de uma ideia de Rosser empregada no fortalecimento do primeiro teorema da incompletude de Gödel [cf., por exemplo, Smullyan, 1992, p. 81], segue-se, da dupla representação de " $x$ é uma sentença atômica verdadeira em $\mathbb{N}$ " 
por $\mathrm{V}_{0}^{\Sigma}(x)$ e $\mathrm{V}_{0}^{\Pi}(x)$, que o conjunto das sentenças atômicas verdadeiras em $\mathbb{N}$ é birepresentável em P.A.. O que contrasta, por exemplo, com o fato que " $x$ é um teorema de P.A." não é bi-representável em P.A..

\section{§3. Introdução à teoria de $\mathrm{V}_{0}(x)$}

Segundo alguns filósofos, Quine entre eles, os predicados de verdade permitiriam enunciados de caráter geral que, em sua ausência, seriam impossíveis de serem estabelecidos [Quine, 1970, pp.10-13].

Grosso modo, se aceitarmos a mais simples das demandas tarskianas, ou seja, que os bicondicionais de Tarski devem valer para um predicado de verdade digno do nome, tal possibilidade de enunciados gerais nem vem ao caso, uma vez que, pelo teorema da indefinibilidade, tais predicados não existem em $L_{P . A .}$. [cf. Indefinibilidade $\mathbf{N}$, p. 23]. Não obstante, desde que estamos munidos da fórmula $\mathrm{V}_{0}(x)$ (se for irrelevante ao argumento, omitiremos os índices ' $\Sigma$ ' e ' $\Pi$ ') e mesmo que o caráter parcial de $\mathrm{V}_{0}(x)$ impeça que esse "predicado" dê conta das demandas tarskianas (o que, nesse caso, está longe de ser um infortúnio), podemos, ainda sim, perguntar pela nossa capacidade de estabelecer algumas generalidades por meio de $\mathrm{V}_{0}(x)^{(1)}$.

Com esse objetivo em mente, não parece ser uma exigência exagerada estabelecer, por exemplo, uma versão formal de "para todo numeral $\tau$, ' $\tau=\tau$ ' é uma sentença atômica verdadeira em $\mathbb{N}$ " ou, referido mais sucintamente, uma versão formal da reflexividade fraca das equações; entretanto, existem alguns problemas em como fazê-lo.

Ilustraremos essas dificuldades mostrando uma pequena armadilha que deve sempre ser evitada.

1. Tratar a fórmula $\mathrm{V}_{0}(x)$ (depois, $\mathrm{V}_{n}(x)$ e, finalmente, $\mathrm{V}(x)$ ) como "predicado de verdade" conduz indiretamente ao problema dos portadores da verdade. Embora $\mathrm{V}_{0}(x)$ seja uma contrapartida formal de um conjunto de números naturais, isso não é tão importante aqui, porque tais números, dadas as contrapartidas formais de outros "predicados" (e. g., " $x$ é uma disjunção" , " $x$ é uma instância de $y$ "), comportam-se como sentenças e não, presumivelmente, como crenças ou proposições. Em todo caso, uma visão bastante tolerante sobre os portadores de verdade pode ser encontrada em Kirkham, 2001, pp. 59-63. 
Sabemos que $\mathrm{NU}(x)$ é uma contrapartida formal de " $x$ é um numeral" [cf. p. 60], de modo que $\forall x\left(\mathrm{NU}(x) \rightarrow \mathrm{V}_{0}(\lceil x=x\rceil)\right)$ poderia ser, à primeira vista, tomada como uma versão formal da reflexividade fraca. Mas, olhando mais atentamente, a "fórmula" $\forall x\left(\mathrm{NU}(x) \rightarrow \mathrm{V}_{0}(\lceil x=x\rceil)\right)$, devido à associação entre a quantificaçãoe a função meta-

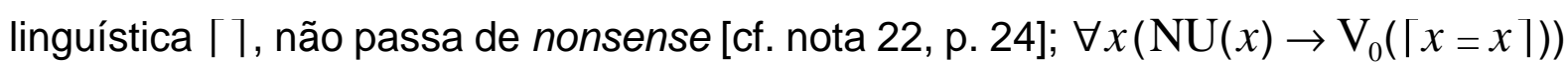
não é uma expressão bem formada de $L_{P . A}-$ e, além disso, não possui nenhum significado metalinguístico claro; embora, contrastantemente, possamos estabelecer que "se $\tau$ for um numeral, então $\mathrm{I} \Sigma_{1}+\mathrm{V}_{0}(\lceil\tau=\tau\rceil)$ ", o que é, obviamente, um enunciado metalinguístico bem construído.

A moral da história é simples: devemos, nesse caso específico e tipicamente em outros similares, ser cuidadosos na escolha das versões formais de nossos enunciados, uma vez que a associação entre quantificação e funções metalinguísticas tende a ser problemática.

Apresentaremos duas (entre várias) possibilidades para uma versão formal de "para todo numeral $\tau, \tau=\tau$ é uma sentença atômica verdadeira em $\mathbb{N}$ ".

\subsection{Descrição da reflexividade fraca das equações.}

a) Escreveremos $\forall n\left(\mathrm{~V}_{0}(\bar{n} \doteq \bar{n})\right)$ no lugar de $\forall x \forall y\left((\mathrm{NU}(x) \wedge \doteq(x, x) \approx y) \rightarrow \mathrm{V}_{0}(y)\right)$;

b) $\mathrm{E} \forall n\left(\mathrm{~V}_{0}(\bar{n} \doteq \bar{n})\right)^{*}$ no lugar de $\forall x \forall y \forall z \forall w((\mathrm{NU}(x) \wedge \doteq(y) \wedge \mathrm{PP} \dot{=}(y) \simeq z \wedge$ $\left.\mathrm{PS} \doteq(y) \simeq z \wedge \operatorname{VAR}(z) \wedge y(z / x) \simeq w) \rightarrow \mathrm{V}_{0}(w)\right)$

Devido à simplicidade, trabalharemos com $\forall n\left(\mathrm{~V}_{0}(\bar{n} \doteq \bar{n})\right)$ como versão formal oficial da reflexividade fraca; mas isso não quer dizer que $\forall n\left(\mathrm{~V}_{0}(\bar{n} \doteq \bar{n})\right)^{*}$ não seja uma versão formal tão boa quanto $\forall n\left(\mathrm{~V}_{0}(\bar{n} \doteq \bar{n})\right)$ desse enunciado; de fato, esperaríamos que essas duas sentenças fossem ambas deriváveis (e, portanto, equivalentes) em I $\Sigma_{1}$ ou, na pior das hipóteses, em P.A.. 
De III 4.1 e 4.3 [cf. p. 60; p. 62] (as subfórmulas relevantes de $\operatorname{TF}(x)$ são $\alpha_{1}$ e $\left.\alpha_{2}\right)$, temos que I $\Sigma_{1} \vdash \forall x(\mathrm{NU}(x) \rightarrow \mathrm{TF}(x))$. Logo, uma vez que 1.2.e) [p. 73], pelas descrições de $\operatorname{LTF}(x) \simeq y$ [p. 64], $\mathrm{V}_{=}(x)$ [pp. 78-79], $\exists s\left(\Phi_{=}(s, x)\right)$ e $\mathrm{V}_{0}$ [p. 82], temos:

3.2. Teorema. $\mathrm{I} \Sigma_{1} \vdash \forall n\left(\mathrm{~V}_{0}(\bar{n} \doteq \bar{n})\right)$.

De modo que, escolhido adequadamente o enunciado correspondente, o teorema 3.2 estabelece uma versão formal da reflexividade fraca. Estamos, assim, diante da primeira generalidade alcançada por meio do predicado $\mathrm{V}_{0}(x)$; e outras generalidades virão, se escolhermos propriamente os enunciados formais adequados.

\subsection{Descrição da reflexividade forte das equações.}

a) Escreveremos $\forall \tau\left(\mathrm{V}_{0}(\tau \doteq \tau)\right)$ no lugar de $\forall x \forall y\left((\mathrm{TF}(x) \wedge \doteq(x, x) \approx y) \rightarrow \mathrm{V}_{0}(y)\right)$.

Basicamente, a mesma argumentação empregada em 3.2 produz:

3.4. Teorema. $\mathrm{I} \Sigma_{1} \vdash \forall \tau\left(\mathrm{V}_{0}(\tau \doteq \tau)\right)$.

Muitas das questões meta-aritméticas mais usuais podem ser respondidas sem um apelo muito minucioso às formas dos enunciados; por exemplo; o primeiro teorema da incompletude de Gödel e "para qualquer termo $\tau, \mathrm{I} \Sigma_{1} \vdash \mathrm{V}_{0}(\lceil\tau=\tau\rceil)$ " podem ser, diferentemente de 3.2, 3.4 e, futuramente, de 3.6, estabelecidos, mobilizando apenas o caráter representativo, respectivamente, da contrapartida formal de " $x$ é um teorema de P.A." e de $\mathrm{V}_{0}(x)$.

Em todo caso, a escolha de $\forall n\left(\mathrm{~V}_{0}(\bar{n} \doteq \bar{n})\right)$, e não de $\forall n\left(\mathrm{~V}_{0}(\bar{n} \doteq \bar{n})\right)^{*}$, para estabelermos a versão formal da reflexividade fraca em 3.2 parece (e, em certa medida, é) arbitrária; devemos apenas definir o que estamos tomando como versão formal do que; entretanto, nosso próximo comentário mostrará que isso não é sempre tão simples e nos conduzirá a outro nível da problemática da escolha dos enunciados, aquela relacionada aos chamados contextos intensionais. 
A análise do argumento de $\mathbf{3 . 2}$ sugere que precisamos do seguinte resultado, à primeira vista trivial, $\operatorname{I} \Sigma_{1} \vdash \operatorname{TF}(x) \rightarrow \exists y(\operatorname{LTF}(x) \simeq y)$. Em nosso caso, esse fato foi pressuposto [cf. 1.2.e), p. 73] (mas poderíamos paciente e rigorosamente estabelecê-lo). Infelizmente, entretanto, existem outras "fórmulas-legenda" que não respeitam 1.2.e).

Por exemplo:

a) Seja $\operatorname{Prf}_{\mathrm{P} . \mathrm{A} .}(x, y)$ uma contrapartida formal de " $x$ é uma prova de $y$ em P.A.".

b) Seja $\operatorname{EPrf}_{\text {P.A. }}(x) \approx y$ uma contrapartida formal da enumeração do conjunto de números de Gödel das provas em P.A..

Assim, $\operatorname{I}_{1} \vdash \operatorname{EPrf}_{\text {P.A. }}(\overline{0}) \approx \bar{k}_{1}, \operatorname{I}_{1} \vdash \operatorname{EPrf}_{\text {P.A. }}(\overline{1}) \approx \bar{k}_{2}, \operatorname{I}_{1} \vdash \operatorname{EPrf}_{\text {P.A. }}(\overline{2}) \approx \bar{k}_{3}$, etc.; de modo que os números de Gödel de todas as provas em P.A. deverão estar entre $k_{1}$, $k_{2}, k_{3}, \ldots$

c) Então, $\operatorname{LTF}(x) \simeq y \wedge \sim \operatorname{Prf}_{\text {P.A. }}\left(\operatorname{EPrf}_{\text {P.A. }}(\operatorname{LTF}(x)),\lceil\overline{0}=\overline{1}\rceil\right)$ ou, mais sucintamente, $\operatorname{LTF}^{\#}(x) \simeq y$ será uma bi-representação em $\Sigma_{1}$ da denotação dos termos fechados (em outras palavras, $\operatorname{LTF}^{\#}(x) \simeq y$ é também uma "fórmula-legenda").

Seja, agora,

d) $\operatorname{TF}^{\#}(x)$ uma abreviação de $\operatorname{TF}(x) \wedge \sim \operatorname{Prf}_{\text {P.A. }}\left(\operatorname{EPrf}_{\text {P.A. }}(\operatorname{LTF}(x)),\lceil\overline{0}=\overline{1}\rceil\right)$;

e) assim, $\operatorname{TF}^{\#}(x) \rightarrow \operatorname{TF}(x)$ será um teorema da lógica.

f) Contudo, $\operatorname{TF}(x) \rightarrow \mathrm{TF}^{\#}(x)$ não é, dado o segundo teorema da incompletude, nem mesmo um teorema de P.A..

Ora, uma vez que, claramente,

g) $\operatorname{I} \Sigma_{1} \vdash \exists y\left(\operatorname{LTF}^{\#}(x) \simeq y\right) \rightarrow \operatorname{TF}^{\#}(x)$;

h) temos, por $\mathbf{g})$ e f), que $\operatorname{TF}(x) \rightarrow \exists y\left(\operatorname{LTF}^{\#}(x) \simeq y\right)$ não é um teorema de P.A. [devemos notar que se $\mathrm{I} \Sigma_{1} \vdash \operatorname{TF}(x) \rightarrow \exists y\left(\operatorname{LTF}^{\#}(x) \simeq y\right)$, então, por $\left.\mathbf{g}\right), \operatorname{I} \Sigma_{1} \vdash \operatorname{TF}(x) \rightarrow$ $\left.\mathrm{TF}^{\#}(x)\right]$;

i) de modo que $\operatorname{LTF}^{\#}(x) \simeq y$ é uma "fórmula-legenda" que não respeita 1.2.e) [cf. p. 73]. 
Em 'Arithmetization of metamathematics in general setting', Solomon Feferman [1960] sustenta uma distinção entre resultados metamatemáticos extensionais e intensionais $^{(2)}$. Grosso modo, uma vez que as contrapartidas formais envolvidas no estabelecimento do primeiro teorema da incompletude de Gödel precisam tão-somente representar aquilo que representam ou, nas palavras de Feferman, "essentially only numerically correct definitions are needed" [ibidem, p. 35], o teorema é um resultado extensional. Enquanto, uma vez que algumas condições restritivas adicionais em relação aos tipos de fórmulas envolvidas são necessárias no caso do segundo teorema da incompletude [cf., por exemplo, ibidem, p.66, “... $\alpha$ is an $R E$-formula ...”], o resultado é intensional $^{(3)}$.

Nossos argumentos, por sua vez, estão fundamentados na maneira concreta como as fórmulas envolvidas são descritas, de modo que condições impostas tão-somente aos tipos de fórmulas seriam possivelmente insuficientes. Poderíamos, então, classificar teoremas nos quais condições intensionais sobre tipos de fórmulas são realmente insuficientes como hiper-intensionais.

Em todo caso, considerações desse tipo, embora obviamente interessantes, estarão além do escopo desse estudo; nossa estratégia será escolher uma fórmula concreta como representante de certa questão meta-aritmética, ignorando, assim,

2. Segundo Feferman [Ibidem, p. 35]: "The applications of the method [of arithmetization] can be classified as being extensional if essentially only numerically correct definitions are needed, or intensional if the definitions must more fully express the notions involved". Baseando-se nessa classificação, Feferman apresenta exemplos de resultados extensionais e intensionais. O primeiro teorema da incompletude de Gödel, o teorema da indefinibilidade (aritmética) das verdades aritméticas de Tarski e os resultados relativos à indecibilidade de teorias do livro Undecidable Theories de Tarski, Mostowski e Robinson, dentre outros, são exemplos de resultados extensionais; enquanto, o segundo teorema da incompletude de Gödel, os resultados relativos à comparação de teorias por meio de provas de consistência relativa e às lógicas ordinais de Turing são exemplos típicos de resultados intensionais.

3. A prova é, basicamente, como se segue: (1) Construímos um enunciado de consistência Con P.A*. $_{\text {a partir de }}$ uma bi-representação "não-canônica" dos axiomas de P.A.; (2) derivamos Con $_{\text {P.A* em P.A.. (3) Ora, o enunciado }}$ de consistência canônico de P.A. não é derivável em P.A.; (4) logo, alguma restrição deve ser imposta as fórmulas empregadas no argumento anterior. (5) Portanto, o segundo teorema da incompletude de Gödel deve ser um resultado intensional. 
justamente dois dos principais problemas colocados no artigo de Feferman: primeiro, o da classificação de questões metamatemáticas como intensionais ou extensionais (mas notemos que estabelecemos logo acima que 1.2.e) [p. 73] é um resultado intensional); segundo, o da possibilidade de um tratamento generalizado ('general setting') de algumas dessas primeiras.

Deixemos de lado essa digressão e retomemos a concretude de nossas fórmulas.

3.5. Descrição da reflexividade das inequações. Escreveremos $\forall \tau\left(\mathrm{V}_{0}(\tau \dot{\leqslant} \tau)\right)$ no lugar de $\forall x \forall y\left((\mathrm{TF}(x) \wedge \dot{\leqslant}(x, x) \approx y) \rightarrow \mathrm{V}_{0}(y)\right)$.

3.6. Teorema. $\mathrm{I} \Sigma_{1} \vdash \forall \tau\left(\mathrm{V}_{0}(\tau \dot{\leqslant} \tau)\right)$.

Vemos assim como a definição de verdade para sentenças atômicas, $\mathrm{V}_{0}$, possibilita enunciados gerais da reflexividade de '=' e ' $\leqslant$ '. Os próximos resultados estabelecem, entre outras coisas, que $V_{0}$ pode realmente ser pensada como uma "definição de verdade para sentenças atômicas".

3.7. Teorema. Se $\alpha$ é uma sentença atômica de $L_{P . A . ~}$ e $\mathbb{N} \vDash \alpha$, então $\mathbb{N} \vDash V_{0}(\lceil\alpha\rceil)$ $\mathrm{e} \mathrm{Q}^{*}+\mathrm{V}_{0}(\lceil\alpha\rceil)$

Prova. Os axiomas "recursivos" da sucessão, soma, multiplicação e exponenciação de Q* permitem "calcular" as denotações de quaisquer termos fechados: basta construirmos uma contrapartida numérica do sequências de valoração dos termos de $\alpha$ por meio da numeração de Gödel.

Por exemplo, para estabelecermos que $\mathrm{Q}^{*}+\mathrm{V}_{0}(\lceil\overline{2}+\overline{2}=\overline{2} \cdot \overline{2}\rceil)$, não precisamos muito mais do que os numerais de Gödel correspondentes às sequências $\langle\overline{0}, 0\rangle,\langle\overline{1}, 1\rangle$, $\langle\overline{2}, 2\rangle,\langle\overline{2}+\overline{2}, 4\rangle$ e $\langle\overline{0}, 0\rangle,\langle\overline{1}, 1\rangle,\langle\overline{2}, 2\rangle,\langle\overline{2} \cdot \overline{2}, 4\rangle$. 
As questões acima são, por assim dizer, positivas e relativamente fáceis de serem respondidas; entretanto, outras questões simples de serem formuladas têm respostas relativamente mais complicadas. Por exemplo:

\subsection{Descrição da injeção entre numerais e legendas.}

a) Escreveremos $\forall n \neq m\left(\sim \mathrm{V}_{0}(\bar{n} \doteq \bar{m})\right)$ no lugar de $\forall x \forall y\left(\left(\mathrm{NU}(x) \wedge \mathrm{NU}(y) \wedge x \neq y \rightarrow \sim \mathrm{V}_{0}(\dot{=}(x, y))\right)\right.$.

3.9. Teorema. $\mathrm{I} \Sigma_{1} \vdash \forall n \neq m\left(\sim \mathrm{V}_{0}(\bar{n} \doteq \bar{m})\right)$.

O teorema 3.9 só pode ser estabelecido na base de uma série de lemas, tais como:

a) Suficiência da decomposição e unicidade da leitura dos termos [cf. pp. 70-71];

b) $\mathrm{I} \Sigma_{1}+\exists y(\operatorname{LTF}(x) \simeq y) \rightarrow \mathrm{TF}(x)$;

c) $\operatorname{I} \Sigma_{1} \vdash(\operatorname{LTF}(x) \simeq \operatorname{LTF}(y) \wedge \mathrm{NU}(x) \wedge \mathrm{NU}(y)) \rightarrow x=y$.

Notemos que, uma vez que $\mathrm{I} \Sigma_{1}+\mathrm{V}_{0}(\lceil\overline{2}=\overline{1}+\overline{1}\rceil) \wedge\lceil\overline{2}\rceil \neq\lceil\overline{1}+\overline{1}\rceil$, um análogo de 3.9 para termos fechados quaisquer é, claramente, falso.

Outro lema útil parece ser:

a) $\mathrm{I} \Sigma_{1} \vdash \forall x(\mathrm{TF}(x) \rightarrow \exists y(\mathrm{NU}(y) \wedge \operatorname{LTF}(x) \simeq \operatorname{LTF}(y))$, que auxiliado pelos lemas anteriores e por 1.2.d) [p. 72] produz:

3.10. Teorema. Se $\alpha$ é uma sentença atômica de $L_{\text {P.A. }}$ tal que $\mathbb{N} \vDash \sim \alpha$, então $\mathbb{N} \vDash \sim \mathrm{V}_{0}(\lceil\alpha\rceil)$ e I $\Sigma_{1} \vdash \sim \mathrm{V}_{0}(\lceil\alpha\rceil)$.

3.11. Corolário. Se $\alpha$ é uma sentença atômica de $L_{P . A}$, então ou temos que $\mathrm{I} \Sigma_{1} \vdash \mathrm{V}_{0}(\lceil\alpha\rceil)$ ou que I $\Sigma_{1} \vdash \sim \mathrm{V}_{0}(\lceil\alpha\rceil)$.

O que é bem diferente do trivial: $I \Sigma_{1} \vdash \mathrm{V}_{0}(\lceil\alpha\rceil) \vee \sim \mathrm{V}_{0}(\lceil\alpha\rceil)$.

3.12. Teorema. Se $\alpha$ é uma sentença atômica de $L_{P . A}$, então $I \Sigma_{1} \vdash \alpha \leftrightarrow V_{0}(\lceil\alpha\rceil)$. 
Apresentaremos, agora, algumas contrapartidas formais da relação entre verdade e derivabilidade.

No que se segue: $\operatorname{Pr}_{\mathrm{Q}^{*}}(x), \operatorname{Pr}_{\mathrm{I} \Sigma_{n}}(x), \operatorname{Pr}_{\mathrm{P} . \mathrm{A} .}(x)$ e $\operatorname{Prf}_{\mathrm{P} . \mathrm{A} .}(x, y)$ são contrapartidas formais de " $x$ é um teorema de $\mathrm{Q}^{*}$ "; " $x$ é um teorema de $\mathrm{I} \Sigma_{n}$ ", onde $\mathrm{I} \Sigma_{n}$ é o resultado de adicionar induções restritas às fórmulas $\mathrm{I} \Sigma_{n}$ aos axiomas de $\mathrm{Q}^{*}$; “ $x$ é um teorema de P.A." e " $x$ é uma derivação de y em P.A.", respectivamente.

3.13. Teorema. $\mathrm{I} \Sigma_{1} \vdash \forall x\left(\mathrm{~V}_{0}(x) \rightarrow \operatorname{Pr}_{\mathrm{Q}^{*}}(x)\right)$. Logo, $\mathrm{I}_{1} \vdash \forall x\left(\mathrm{~V}_{0}(x) \rightarrow \operatorname{Pr}_{\text {P.A. }}(x)\right)$, uma vez que $\mathrm{I} \Sigma_{1} \vdash \forall x\left(\operatorname{Pr}_{\mathrm{Q}}(x) \rightarrow \operatorname{Pr}_{\text {P.A. }}(x)\right)$.

É instrutivo notar que $\mathrm{I}_{1}+\operatorname{Pr}_{\mathrm{Q}^{*}}(\lceil\overline{1}=\overline{2} \rightarrow \overline{1}=\overline{2}\rceil) \wedge \sim \mathrm{V}_{0}(\lceil\overline{1}=\overline{2} \rightarrow \overline{1}=\overline{2}\rceil)$, uma vez que $\mathrm{V}_{0}$ está restrita às sentenças atômicas.

No caso da "conversa" de 3.13, a história é bem diferente. De 3.11 e do segundo teorema da incompletude de Gödel, temos (se P.A. é consistente):

3.14. Teorema. P.A. $\forall \forall x\left(\operatorname{Pr}_{\text {P.A. }}(x) \wedge \operatorname{SENT}_{0}(x) \rightarrow \mathrm{V}_{0}(x)\right) \mathrm{e}$, mais genericamente, $\mathrm{I}_{n} H \forall x\left(\operatorname{Pr}_{I_{n}}(x) \wedge \operatorname{SENT}_{0}(x) \rightarrow \mathrm{V}_{0}(x)\right)$.

Prova (esboço). De 3.11, temos P.A. $\vdash \mathrm{V}_{0}(\lceil\overline{0}=\overline{1}\rceil)$ ou P.A. $\vdash \sim \mathrm{V}_{0}(\lceil\overline{0}=\overline{1}\rceil)$.

No primeiro caso, se P.A. $\vdash \mathrm{V}_{0}(\lceil\overline{0}=\overline{1}\rceil)$, por 3.13, temos que P.A. $\vdash \operatorname{Pr}_{\text {P.A. }}(\lceil\overline{0}=\overline{1}\rceil)$ e, daí, que P.A. é inconsistente.

No segundo, temos que P.A. $\vdash \sim \mathrm{V}_{0}(\lceil\overline{0}=\overline{1}\rceil)$ e P.A. $\vdash \operatorname{SENT}_{0}(\lceil\overline{0}=\overline{1}\rceil)$.

Assim, da suposição que P.A. $\vdash \forall x\left(\operatorname{Pr}_{\text {P.A. }}(x) \wedge \operatorname{SENT}_{0}(x) \rightarrow \mathrm{V}_{0}(x)\right)$ se segue que P.A. $\vdash \sim \operatorname{Pr}_{\text {P.A. }}(\lceil\overline{0}=\overline{1}\rceil)$, o que é contrário ao segundo teorema da incompletude.

Como é comum em meta-aritmética, temos "aproximações positivas" do resultado negativo acima:

3.15. Teorema. Para qualquer $n$, P.A. $\vdash \forall x\left(\operatorname{Prf}_{\text {P.A. }}(\bar{n}, x) \wedge \operatorname{SENT}_{0}(x) \rightarrow \mathrm{V}_{0}(x)\right)$. 
As definições parciais de verdade permitem que enunciemos questões interessantes sobre a consistência relativamente a P.A. e seus fragmentos ou aos próprios fragmentos de P.A..

\subsection{Questões.}

a) Para quais $n$ 's, P.A. $\vdash \forall x\left(\operatorname{Pr}_{I_{n}}(x) \wedge \operatorname{SENT}_{0}(x) \rightarrow \mathrm{V}_{0}(x)\right)$;

b) Para quais $n$ 's e $m$ 's, $\mathrm{I}_{n} \vdash \forall x\left(\operatorname{Pr}_{\mathrm{I} \Sigma_{m}}(x) \wedge \operatorname{SENT}_{0}(x) \rightarrow \mathrm{V}_{0}(x)\right)$ ?

Desde que temos a reflexividade de P.A. (ou seja, P.A. estabelece a consistência de seus fragmentos finitos) [cf., por exemplo, Feferman, 1960, pp. 67-68] e o fato que os fragmentos I $\Sigma_{n}$ são finitamente axiomatizáveis [cf., Hájek \& Pudlák, 1998, pp. 77-81], a resposta da primeira pergunta parece ser: para todos os n's; mas, como é usual, construir o argumento dentro de P.A. não parece ser tarefa fácil.

\section{§4. Outros tipos de definições parciais de verdade}

As fórmulas-legenda $\operatorname{LTM}^{\Sigma}(x, y) \simeq z$ e $\operatorname{LTM}^{\Pi}(x, y) \simeq z$, esboçadas no final da seção 5 do capítulo III [p. 65] e baseadas na atribuição de valores às variáveis livres de termos abertos por meio de sequências, permitem construções alternativas de contrapartidas formais dos predicados parciais de verdade.

No caso, estabeleceríamos, primeiramente, uma "relação de satisfação" aplicável apenas às fórmulas atômicas de $\mathrm{L}_{\text {P.A. }}$; no caso, fórmulas atômicas (abertas incluídas) seriam satisfeitas por uma determinada sequência de números naturais. Construiríamos, então, à maneira de Tarski, definições de verdade $\Sigma_{1}$ e $\Pi_{1}$ de fórmulas atômicas.

Além disso, poderíamos, tanto por meio de "relações de satisfação" quanto, mais diretamente, por meio das fórmulas-legenda para termos fechados, estabelecer uma base ligeiramente diferente para a hierarquia dos predicados parciais de verdade. Nesse caso, contruiríamos definições de verdade $\mathrm{V}_{\Sigma_{0}}^{\Sigma}(x)$ e $\mathrm{V}_{\Sigma_{0}}^{\Pi}(x)$, respectivamente, $\Sigma_{1}$ e $\Pi_{1}$ para fórmulas $\Sigma_{0}$ [cf. Hájek \& Pudlak, 1998, pp. 56-58]. 
O importante aqui é que essa construção é possível e que, embora as fórmulas $\Sigma_{0}$ sejam logicamente mais complexas que as fórmulas atômicas, não o são do ponto de vista da hierarquia da aritmética.

Devemos notar, entre outras coisas, que:

4.1. Propriedades básicas de $\mathrm{V}_{\Sigma_{0}}^{\Sigma}(x)$ e $\mathrm{V}_{\Sigma_{0}}^{\Pi}(x)$.

a) $\mathrm{V}_{\Sigma_{0}}^{\Sigma}(x)$ é $\Sigma_{1}$ e a única variável livre de $\mathrm{V}_{\Sigma_{0}}^{\Sigma}(x)$ é $x$;

b) $\mathrm{V}_{\Sigma_{0}}^{\Pi}(x)$ é $\Pi_{1}$ e a única variável livre de $\mathrm{V}_{\Sigma_{0}}^{\Pi}(x)$ é $x$;

c) $\mathrm{I} \Sigma_{1} \vdash \forall x\left(\mathrm{~V}_{\Sigma_{0}}^{\Sigma}(x) \leftrightarrow \mathrm{V}_{\Sigma_{0}}^{\Pi}(x)\right)$.

d) Se $\alpha$ é uma sentença $\Sigma_{0}$ tal que $\mathbb{N} \vDash \alpha$, então $I \Sigma_{1} \vdash V_{\Sigma_{0}}(\lceil\alpha\rceil)$;

e) Se $\mathrm{I} \Sigma_{1} \vdash \mathrm{V}_{\Sigma_{0}}(\bar{n})$, então $g^{-1}(n)$ é uma sentença $\Sigma_{0}$ e $\mathbb{N} \vDash g^{-1}(n)$;

f) $\mathrm{I}_{1} \vdash \forall x\left(\mathrm{~V}_{0}(x) \rightarrow \mathrm{V}_{\Sigma_{0}}(x)\right) \wedge \exists x\left(\sim \mathrm{V}_{0}(x) \wedge \mathrm{V}_{\Sigma_{0}}(x)\right)$.

\section{§5. Definição de verdade para sentenças de complexidade $n$}

Nosso primeiro problema é descrever uma contrapartida formal do predicado de verdade para sentenças de complexidade 1 por meio de $\mathrm{V}_{0}^{\Sigma}(x)$ e $\mathrm{V}_{0}^{\Pi}(x)$ conjuntamente com o lugar que esse predicado ocupa na hierarquia da aritmética.

\subsection{Definição de verdade para sentenças de complexidade 1 .}

a) Escreveremos $V_{1}(x)$ no lugar de $\alpha_{1} \vee \alpha_{2} \vee \alpha_{3} \vee \alpha_{4} \vee \alpha_{5} \vee \alpha_{6}$;

b) Onde $\alpha_{1}$ é $\mathrm{V}_{0}^{\Sigma}(x)$;

c) $\alpha_{2}$ é $\left(\operatorname{SENT} \dot{\sim}_{1}(x) \wedge \sim \mathrm{V}_{0}^{\Pi}(\mathrm{P} \dot{\sim}(x))\right)$;

d) $\alpha_{3}$ é $\left(\operatorname{SENT} \dot{\vee}_{1}(x) \wedge\left(\mathrm{V}_{0}^{\Sigma}(\operatorname{PP} \dot{\vee}(x)) \vee \mathrm{V}_{0}^{\Sigma}(\operatorname{PS} \dot{\vee}(x))\right)\right)$;

e) $\alpha_{4}$ é $\left(\operatorname{SENT} \dot{\lambda}_{1}(x) \wedge\left(\mathrm{V}_{0}^{\Sigma}(\mathrm{PP} \dot{\wedge}(x)) \wedge \mathrm{V}_{0}^{\Sigma}(\operatorname{PS} \dot{\lambda}(x))\right)\right)$;

f) $\alpha_{5}$ é $\left(\operatorname{SENT} \dot{\exists}_{1}(x) \wedge \exists y \mathrm{~V}_{0}^{\Sigma}(\operatorname{INS}(x, y))\right)$;

g) $\alpha_{6}$ é $\left(\operatorname{SENT} \dot{\forall}_{1}(x) \wedge \forall y \mathrm{~V}_{0}^{\Pi}(\operatorname{INS}(x, y))\right)$. 
Obviamente, $\mathrm{V}_{1}(x)$ não é uma fórmula da hierarquia da aritmética; entretanto, $\mathrm{V}_{1}(x)$ pode ser posta em uma forma normal prenex $\mathrm{NP}\left[\mathrm{V}_{1}(x)\right]$ tal que $\vdash \mathrm{V}_{1}(x) \leftrightarrow \mathrm{NP}\left[\mathrm{V}_{1}(x)\right] \mathrm{e}$, depois da contração dos quantificadores, em uma forma "normal aritmética" $\mathrm{NA}\left[\mathrm{V}_{1}(x)\right]$ tal que $\mathrm{I} \Sigma_{0} \vdash \mathrm{V}_{1}(x) \leftrightarrow \mathrm{NA}\left[\mathrm{V}_{1}(x)\right]$. Infelizmente, o processo tal como descrito não pode estabelecer univocamente $\mathrm{NA}\left[\mathrm{V}_{1}(x)\right]$ nem, portanto, a posição de $\mathrm{NA}\left[\mathrm{V}_{1}(x)\right]$ na hierarquia aritmética. Devemos, então, analisar $\mathrm{V}_{1}(x)$ mais de perto.

A estrutura de $V_{1}(x)$ relevante para análise é $\left.\left(\left(\left(\left(\alpha_{1} \vee \alpha_{2}\right) \vee \alpha_{3}\right) \vee \alpha_{4}\right) \vee \alpha_{5}\right) \vee \alpha_{6}\right)$.

Começaremos "reduzindo" $\alpha_{2}$ a uma fórmula $\Sigma_{1}$ :

$\mathrm{V}_{0}^{\Pi}(x)$ é $\Pi_{1}$.

Assim, segundo nossas convenções anteriores [p. 60]:

$\mathrm{V}_{0}^{\Pi}(\mathrm{P} \sim(x))$ é $\forall y\left(\mathrm{P} \dot{\sim}(x) \simeq y \rightarrow \mathrm{V}_{0}^{\Pi}(y)\right)$.

Ora, $\mathrm{V}_{0}^{\Pi}(y)$ é $\forall s\left(\varpi_{0}(s, y)\right)$, onde $\varpi_{0}(s, y)$ é $\Sigma_{0}$.

Temos, assim, que $\vdash\left(\forall y\left(\mathrm{P} \dot{\sim}(x) \simeq y \rightarrow \mathrm{V}_{0}^{\Pi}(y)\right)\right) \leftrightarrow \forall y \forall s\left(\mathrm{P} \dot{\sim}(x) \simeq y \rightarrow \varpi_{0}(s, y)\right)$, para uma escolha adequada da variável $s$, e que $\mathrm{I} \Sigma_{1} \vdash \forall y \forall s\left(\mathrm{P} \dot{\sim}(x) \simeq y \rightarrow \varpi_{0}(s, y)\right) \leftrightarrow$ $\left.\forall y\left(\mu_{2}(y, x)\right)\right)$, onde $\left.\forall y\left(\mu_{2}(y, x)\right)\right)$ é $\forall y \forall v_{i}<y \forall v_{j}<y\left(\operatorname{COMP}(y) \simeq \overline{2} \wedge(y)_{1} \simeq v_{i} \wedge(y)_{2} \simeq v_{j}\right.$ $\left.\rightarrow\left[\mathrm{P} \dot{\sim}(x) \simeq y \rightarrow \varpi_{0}(s, y)\right] s / v_{i} y / v_{j}\right)$ e, portanto, é $\Pi_{1}$.

Obviamente, $\vdash \sim \forall y\left(\mu_{2}(y, x)\right) \leftrightarrow \exists y\left(\sim \mu_{2}(y, x)\right)$, cuja subfórmula da direita é $\Sigma_{1}$. Logo, uma vez que SENT $\dot{\sim}_{1}(x)$ é $\Sigma_{0}$, temos que I $\Sigma_{1} \vdash \alpha_{2} \leftrightarrow \exists z\left(\operatorname{SENT} \dot{\sim}_{1}(x) \wedge\right.$ $\sim \mu_{2}(z, x)$ ), onde $\exists z\left(\operatorname{SENT}_{1}(x) \wedge \sim \mu_{2}(z, x)\right)$ ou $\exists z\left(\sigma_{2}(z, x)\right)$ é a redução $\Sigma_{1}$ de $\alpha_{2}$.

Passemos para $\alpha_{3}$.

$\mathrm{V}_{0}^{\Sigma}(\mathrm{PP} \dot{\vee}(x))$ e $\mathrm{V}_{0}^{\Sigma}(\mathrm{PS} \dot{\vee}(x))$ são $\Sigma_{1}$. Por exemplo, $\mathrm{V}_{0}^{\Sigma}(\mathrm{PP} \dot{\vee}(x))$ é $\exists y(\operatorname{PP} \dot{\vee}(x) \simeq y$ $\left.\wedge \mathrm{V}_{0}^{\Sigma}(y)\right)$

Os passos da redução são os seguintes: 1) os quanticadores existenciais de $\mathrm{V}_{0}^{\Sigma}(\operatorname{PP} \dot{\mathrm{V}}(x))$ e $\left.\mathrm{V}_{0}^{\Sigma}(\operatorname{PS} \dot{\mathrm{V}}(x))\right)$ são postos "para fora" das fórmulas; 2$)$ "formamos" a disjunção das fórmulas de $1 ; 3$ ) os quantificadores da disjunção de 2 são postos 
novamente "para fora"; 4) "contraímos" os quantificadores de 3; 5) "formamos" a conjunção de $\operatorname{SENT} \dot{v}_{1}(x)$ e da fórmula de $\left.4 ; 6\right)$ o quantificador de 5 é posto "para fora". Temos, então, que I $\Sigma_{0} \vdash \alpha_{3} \leftrightarrow \exists z\left(\operatorname{SENT} \dot{\vee}_{1}(x) \wedge \mu_{3}(z, x)\right)$, onde $\exists z\left(\operatorname{SENT} \dot{\vee}_{1}(x) \wedge \mu_{3}(z, x)\right)$ ou $\exists z\left(\sigma_{3}(z, x)\right)$, ou seja, o resultado do passo 6 é a redução $\Sigma_{1}$ de $\alpha_{3}$.

Devemos reduzir, então, $\alpha_{4}$ e $\alpha_{5}$ a $\exists z\left(\sigma_{4}(z, x)\right)$ e $\exists z\left(\sigma_{5}(z, x)\right)$ pelo mesmo processo dos parágrafos anteriores.

No caso de $\alpha_{6}$, a ideia é partir de $V_{0}^{\Pi}(x)$ sempre "buscando" reduções $\Pi_{1}$ das demais subfórmulas de $\alpha_{6}$ que são "superfórmulas" de $\mathrm{V}_{0}^{\Pi}(x)$.

Temos, então, reduções $\Sigma_{1}$ de $\alpha_{1}, \alpha_{2}, \alpha_{3}, \alpha_{4}$ e $\alpha_{5}$, além de uma redução $\Pi_{1}$ $\forall z\left(\sigma_{6}(z, x)\right)$ de $\alpha_{6}$. Assim, $\mathrm{I}_{1} \vdash \mathrm{V}_{1}(x) \leftrightarrow\left(\left(\mathrm{V}_{0}^{\Sigma}(x) \vee \exists z\left(\sigma_{2}(z, x)\right) \vee \exists z\left(\sigma_{3}(z, x)\right) \vee \exists z\left(\sigma_{4}(z\right.\right.\right.$, $\left.\left.x)) \vee \exists z\left(\sigma_{5}(z, x)\right)\right) \vee \forall z\left(\sigma_{6}(z, x)\right)\right)$. Finalmente, reduzimos as subfórmulas $\Sigma_{1}$ da subfórmula direita da "redução" acima, de modo que $\mathrm{I} \Sigma_{1} \vdash \mathrm{V}_{1}(x) \leftrightarrow \exists z\left(\sigma_{1-5}(z, x) \vee \forall z\left(\sigma_{6}(z, x)\right)\right)$, onde $\sigma_{1-5}(z, x)$ é $\left(\mathrm{V}_{0}^{\Sigma}(x) \vee \sigma_{2}(z, x) \vee \sigma_{3}(z, x) \vee \sigma_{4}(z, x) \vee \sigma_{5}(z, x)\right)$.

Podemos, então, apresentar versões $\Sigma_{2}$ e $\Pi_{2}$ de $\mathrm{V}_{1}(x)$.

\subsection{Definições de verdade para sentenças de complexidade 1.}

a) $\mathrm{V}_{1}^{\Sigma}(x)$ é abreviação de $\left.\exists z \forall w\left(\sigma_{1-5}(z, x)\right) \vee \sigma_{6}(w, x)\right)$;

c) $\mathrm{V}_{1}^{\Pi}(x)$ é abreviação de $\left.\forall z \exists w\left(\sigma_{1-5}(w, x)\right) \vee \sigma_{6}(z, x)\right)$.

Mais geralmente, temos que:

5.3. Definição de verdade para sentenças de complexidade $n+1$.

a) Escreveremos $V_{n+1}(x)$ no lugar de $\alpha_{1} \vee \alpha_{2} \vee \alpha_{3} \vee \alpha_{4} \vee \alpha_{5} \vee \alpha_{6}$;

b) Onde $\alpha_{1}$ é $\mathrm{V}_{n}^{\Sigma}(x)$;

c) $\alpha_{2}$ é $\left(\operatorname{SENT}_{\sim_{n+1}}(x) \wedge \sim \mathrm{V}_{n}^{\Pi}(\mathrm{P} \sim(x))\right)$;

d) $\alpha_{3}$ é $\left(\operatorname{SENT} \dot{\vee}_{n+1}(x) \wedge\left(\mathrm{V}_{n}^{\Sigma}(\mathrm{PP} \dot{\vee}(x)) \vee \mathrm{V}_{n}^{\Sigma}(\mathrm{PS} \dot{\vee}(x))\right)\right)$;

e) $\alpha_{4}$ é $\left(\operatorname{SENT} \dot{\wedge}_{n+1}(x) \wedge\left(\mathrm{V}_{n}^{\Sigma}(\mathrm{PP} \dot{\wedge}(x)) \wedge \mathrm{V}_{n}^{\Sigma}(\mathrm{PS} \dot{\wedge}(x))\right)\right)$; 
f) $\alpha_{5}$ é $\left(\operatorname{SENT} \dot{\exists}_{n+1}(x) \wedge \exists y \mathrm{~V}_{n}^{\Sigma}(\operatorname{INS}(x, y))\right)$;

g) $\alpha_{6}$ é $\left(\operatorname{SENT} \dot{\nabla}_{n+1}(x) \wedge \forall y \mathrm{~V}_{n}^{\Pi}(\operatorname{INS}(x, y))\right)$.

A estrutura relevante de $\mathrm{V}_{n_{+1}}(x)$ para análise é essencialmente a mesma de $\mathrm{V}_{1}(x)$ $\left.\left(\left(\left(\left(\alpha_{1} \vee \alpha_{2}\right) \vee \alpha_{3}\right) \vee \alpha_{4}\right) \vee \alpha_{5}\right) \vee \alpha_{6}\right)$ e as "reduções" são realizadas em etapas similirares.

No caso $\alpha_{2}, \mathrm{~V}_{n}^{\Pi}(x)$ é $\Pi_{1} ;$ portanto, $\mathrm{V}_{n}^{\Pi}(\mathrm{P} \dot{\sim}(x))$ é $\forall y\left(\mathrm{P} \sim(x) \simeq y \rightarrow \mathrm{V}_{n}^{\Pi}(y)\right)$. Ora, $\mathrm{V}_{n}^{\Pi}(y)$ é $\forall s_{n} \exists s_{n^{-1}} \cdots\left(\varpi_{0}\left(s_{n}, s_{n^{-1}}, \ldots, y\right)\right)$, onde $\varpi_{0}\left(s_{n}, s_{n^{-1}}, \ldots, y\right)$ é $\Sigma_{0}$. Assim, uma vez que $\vdash\left(\forall y\left(\mathrm{P} \dot{\sim}(x) \simeq y \rightarrow \mathrm{V}_{n}^{\Pi}(y)\right)\right) \leftrightarrow \forall y \forall s_{n} \exists s_{n^{-1}} \cdots\left(\mathrm{P} \dot{\sim}(x) \simeq y \rightarrow \varpi_{0}\left(s_{n}, s_{n-1}, \ldots, y\right)\right)$, para escolhas adequadas de variáveis, podemos, então,"contrair" ' $\forall y \forall s_{n}$ ' em I $\Sigma_{1}$ ficando com uma fórmula $\prod_{n}$, abreviada por $\forall s_{n} \exists s_{n-1} \cdots\left(\mu_{2}\left(s_{n}, s_{n-1}, \ldots, x\right)\right)$, cuja negação equivale logicamente a $\exists s_{n} \forall s_{n-1} \cdots\left(\sim \mu_{2}\left(s_{n}, s_{n-1}, \ldots, x\right)\right)$ (uma fórmula $\left.\Sigma_{n}\right)$. Uma vez que $\operatorname{SENT} \dot{\sim}_{1}(x)$ é $\Sigma_{0}$, temos que I $\Sigma_{1} \vdash \alpha_{2} \leftrightarrow \exists s_{n} \forall s_{n-1} \cdots\left(\operatorname{SENT} \dot{\sim}_{1}(x) \wedge \sim \mu_{2}\left(s_{n}, s_{n-1}, \ldots, x\right)\right)$, cuja subfórmula da direita ou $\exists s_{n} \forall s_{n^{-1}} \cdots\left(\sigma_{2}\left(s_{n}, s_{n-1}, \ldots, x\right)\right)$ será a redução $\Sigma_{n}$ de $\alpha_{2}$.

Podemos, então, aplicar procedimentos similares em $\alpha_{3}, \alpha_{4}, \alpha_{5}$ e $\alpha_{6}$, o que produzirá, eventualmente, reduções $\Sigma_{n}$ nos três primeiros casos e uma redução $\Pi_{n}$ no último. Podemos, agora, reduzir sucessivamente as disjunções de $\mathrm{V}_{n_{+1}}(x)$; devemos, para tanto, 1) "exteriorizar" os quantificadores de cada disjunto alternadamente e, depois, 2) "contrair" (em I $\Sigma_{1}$ ) os pares de quantificadores universais e existenciais.

Uma vez reduzidos os cinco primeiros disjuntos de $\mathrm{V}_{n_{+1}}(x)$, teremos algo como: $\mathrm{I} \Sigma_{1} \vdash \mathrm{V}_{n+1}(x) \leftrightarrow \exists s_{n} \forall s_{n-1} \cdots\left(\sigma_{1-5}\left(s_{n}, \ldots, x\right)\right) \vee \forall s_{n} \exists s_{n-1} \cdots\left(\operatorname{SENT} \dot{\forall}_{1}(x) \wedge \mu_{6}\left(s_{n}, \ldots, x\right)\right)$.

Devemos produzir, finalmente, versões $\Sigma_{n+1}$ e $\Pi_{n+1}$ de $\mathrm{V}_{n}(x)$, abreviadas por $\mathrm{V}_{n}^{\Sigma}$ $\mathrm{e} \mathrm{V}_{n}^{\Pi}$, conforme escolhemos o primeiro quantificador a ser "exteriorizado".

Sabemos que é impossível apresentar uma única fórmula de $L_{P . A}$ que descreva o comportamento de toda sequência de definições parciais de verdade (o que é uma 
consequência da Indefinibilidade $\mathbb{N}$ [cf. p. 23; p. 37]); no entanto, acabamos de mostrar como sucessivamente construir essas definições parciais.

Devemos notar que $\mathrm{V}_{0}(x), \mathrm{V}_{1}(x), \mathrm{V}_{2}(x), \ldots, \mathrm{V}_{n}(x), \ldots$ é uma sequência de fórmulas de $L_{\text {P.A. }}$, de modo que ' $n$ ' em $\mathrm{V}_{n}$ é, bem-entendido, um parâmetro metalinguístico e não uma variável de LP.A. [cf. discussão p. 37].

\section{§6. Introdução à teoria das definições parciais de verdade}

Apresentaremos nessa seção alguns resultados da teoria das definições parciais de verdade ou DPVs tanto do ponto de vista da generalidade quineana [cf. p. 83] quanto da adequação tarskiana [cf. pp. 24-25].

Dada a construção das DPVs da seção anterior, o teorema abaixo é um resultado totalmente trivial.

6.1. Teorema. $\vdash \forall x\left(\mathrm{~V}_{n}(x) \rightarrow \mathrm{V}_{n+1}(x)\right)$, para todo $n$.

Além disso, podemos notar algumas similaridades estruturais entre as definições parciais e as contrapartidas formais da derivabilidade nos subsistemas de P.A.; temos, por exemplo, que I $\Sigma_{1} \vdash \forall x\left(\operatorname{Pr}_{I_{n}}(x) \rightarrow \operatorname{Pr}_{I_{n+1}}(x)\right) \wedge \forall x\left(\operatorname{Pr}_{\mathrm{II}{ }_{n}}(x) \rightarrow \operatorname{Pr}_{\mathrm{I}_{n+1}}(x)\right)$, embora tal resultado não seja totalmentre trivial.

Outro resultado trivial é:

6.2. Teorema. $\mathrm{I} \Sigma_{1} \vdash \exists x\left(\mathrm{~V}_{n+1}(x) \wedge \sim \mathrm{V}_{n}(x)\right)$.

E, também, nesse caso, parece que temos, para sistemas formais suficientemente fortes, que $\exists x\left(\operatorname{Pr}_{\mathrm{I} \Sigma_{n+1}}(x) \wedge \sim \operatorname{Pr}_{\mathrm{I} \Sigma_{n}}(x)\right)$; contudo, tal resultado está, pelo menos à primeira vista, longe de ser trivial. Em Hájek e Pudlak [1998, p. 220 e segs.], por exemplo, o resultado informal correspondente, ou seja, "I $\Sigma_{n+1}$ é dedutivamente mais forte que $\mathrm{I} \Sigma_{n}(x)$ " é estabelecido por meio de considerações modelo-teoréticas e a formalização desse tipo 
de argumento é, em geral, bastante complicada. Obviamente, a possibilidade de argumentos alternativos fica aberta e seria, de fato, interessante explorá-las.

Minha aposta seria em algum tipo de "sentença de Rosser" específica para cada fragmento de P.A. (talvez, versões "finitizadas" dos esquemas de indução relativos aos fragmentos [cf. ibidem, p. 78] possam funcionar); entretanto, isso nos levaria bem longe dos objetivos desse estudo.

O resultado mais importante dessa seção é, não surpreendentemente, uma versão parametrizada dos bicondicionais de Tarski:

6.3. Teorema. Para qualquer número natural $n$ dado, se $\alpha$ é uma sentença de $\mathrm{L}_{\text {P.A. }}$ cuja complexidade é $n$, então P.A. $\vdash \alpha \leftrightarrow \mathrm{V}_{n}(\lceil\alpha\rceil)$.

Por meio de lemas versando sobre a estrutura das sentenças e das definições parciais, podemos estabelecer versões genéricas (parametrizadas) das condições de verdade tarskianas:

6.4. Condições tarskianas para $\mathrm{V}_{n}(x)$ e $\mathrm{V}_{n+1}(x)$.

a) $\mathrm{I}_{1} \vdash \forall x\left(\operatorname{SENT}_{n}(x) \wedge \sim \mathrm{V}_{n}(x) \rightarrow \mathrm{V}_{n+1}(\dot{\sim}(x))\right)$;

b) $\mathrm{I}_{1} \vdash \forall x \forall y\left(\mathrm{~V}_{m}(x) \wedge \operatorname{SENT}_{n}(y) \rightarrow \mathrm{V}_{m+n}(\dot{\vee}(x, y)) \wedge \mathrm{V}_{m+n}(\dot{\vee}(y, x))\right)$;

c) $\mathrm{I} \Sigma_{1} \vdash \forall x \forall y\left(\mathrm{~V}_{m}(x) \wedge \mathrm{V}_{n}(y) \rightarrow \mathrm{V}_{m+n}(\dot{\wedge}(x, y))\right)$;

d) $\mathrm{I}_{1} \vdash \forall x\left(\exists y\left(\mathrm{~V}_{n}(\operatorname{INS}(x, y))\right) \rightarrow \mathrm{V}_{n+1}(\dot{\exists}(x))\right)$;

e) $\mathrm{I}_{1} \vdash \forall x\left(\forall y\left(\mathrm{~V}_{n}(\operatorname{INS}(x, y))\right) \rightarrow \mathrm{V}_{n+1}(\dot{\forall}(x))\right)$.

Menos trivialmente, por meio da suficiência da decomposição, da unicidade de leitura das sentenças e da estrutura das DPVs, temos algumas formas "conversas" das condições tarskianas, por exemplo:

\subsection{Conversas das condições tarskianas para $\mathrm{V}_{n}(x)$.}

a) $\mathrm{I} \Sigma_{1} \vdash \forall x\left(\mathrm{~V}_{n}(\dot{\sim}(x)) \rightarrow \sim \mathrm{V}_{n}(x)\right)$; 
b) $\mathrm{I} \Sigma_{1} \vdash \forall x \forall y\left(\mathrm{~V}_{n}(\dot{\vee}(x, y)) \rightarrow \mathrm{V}_{n}(x) \vee \mathrm{V}_{n}(y)\right)$;

c) $\mathrm{I}_{1} \vdash \forall x\left(\mathrm{~V}_{n}(\dot{\exists}(x)) \rightarrow \exists y\left(\mathrm{~V}_{n}(\operatorname{INS}(x, y))\right)\right)$.

De modo que um manejo cuidadoso dos índices das DPVs possibilita versões "semânticas" de outros teoremas da lógica, por exemplo, $\mathrm{I}_{1} \vdash \forall x\left(\mathrm{~V}_{n}(\dot{\sim}(\dot{\sim}(x))) \rightarrow \mathrm{V}_{n}(x)\right)$ (princípio de eliminação da dupla negação).

\section{§7. Definições parciais de falsidade}

Analogamente às definições parciais de verdade, podemos introduzir definições parciais de falsidade ou, mais sucintamente, DPFs. Iremos supor que o fizemos adequadamente, de modo que teremos resultados tanto análogos quanto "duais" àqueles das seções §2, §3 e §6 desse capítulo.

Por exemplo, podemos apresentar, analogamente à descrição 2.5 [cf. p. 78], uma definição $\Sigma_{1}$ da "falsidade de equações":

\subsection{Descrição semi-formal $\Sigma_{1}$ da falsidade em $\mathbb{N}$ para equações.}

a) Escreveremos $\mathrm{F}_{=}^{\Sigma}(x)$ no lugar de

$\mathrm{SENT} \doteq(x) \wedge \sim\left(\operatorname{LTF}^{\Pi}(\mathrm{PP} \doteq(x))=\operatorname{LTF}^{\Pi}(\mathrm{PS} \doteq(x))\right)$.

Podemos, além disso, produzir análogos e duais dos resultados anteriores, por exemplo:

7.2. Algumas propriedades de $\mathrm{F}_{n}(x)$ e $\mathrm{F}_{n+1}(x)$.

a) Para qualquer número natural $n$ dado, se $\alpha$ é uma sentença de $L_{\text {P.A. }}$ cuja complexidade é $n$, então P.A. $\vdash \sim \alpha \leftrightarrow \mathrm{F}_{n}([\alpha])$ [cp. p. 97];

b) $\vdash \forall x\left(\mathrm{~F}_{n}(x) \rightarrow \mathrm{F}_{n+1}(x)\right)$, para todo $n$ [cp. p. 97];

c) $\mathrm{I}_{1} \vdash \forall x\left(\operatorname{SENT}_{n}(x) \wedge \sim \mathrm{F}_{n}(x) \rightarrow \mathrm{F}_{n+1}(\dot{\sim}(x))\right)$ [cp. p. 97]. 
Outra aplicação interessante das DPFs dizem respeito à interação entre elas e as definições parciais de verdade:

7.3. Algumas Propriedades de $\mathrm{F}_{n}(x)$ e $\mathrm{V}_{n}(x)$.
a) $\mathrm{I}_{1} \vdash \forall x\left(\mathrm{~F}_{n}(x) \rightarrow \mathrm{V}_{n+1}(\dot{\sim}(x))\right)$;
b) $\mathrm{I}_{1} \vdash \forall x\left(\mathrm{~V}_{n}(x) \rightarrow \mathrm{F}_{n+1}(\dot{\sim}(x))\right)$;
c) $\mathrm{I}_{1} \vdash \forall x\left(\operatorname{SENT}_{n}(x) \rightarrow \mathrm{F}_{n}(x) \vee \mathrm{V}_{n}(x)\right)$. 


\section{V}

\section{Alguns sistemas de acumulação}

Embora as considerações anteriores sejam compreensivelmente incompletas, esperamos que o capítulo IV proporcione subsídios suficientes, de um lado, para estabelecermos a correção dos sistemas desse capítulo, de outro, para lhes avaliarmos a adequação. De fato, dado o significado intuitivo das definições parciais de verdade (DPVs), ou seja, dado que as DPVs realmente expressam o que deveriam expressar, é bastante óbvio que nossos sistemas serão todos corretos [cf. p. 36]. Resta-nos, portanto, defini-los adequadamente, examiná-los e compará-los com outros sistemas já propostos na literatura especializada.

Infelizmente, algumas limitações de conhecimento (e. g., minha ignorância sobre várias das técnicas mais avançadas utilizadas tanto na teoria da derivação quanto na teoria dos modelos) e de tempo (e. g., muito da discussão demanda um desenvolvimento mais completo da aritmética das contrapartidas formais que propomos anteriormente) inviabilizam um tratamento satisfatório de todas as questões que poderiam ser colocadas. Contudo, acreditamos que o que se segue é um bom começo e, obviamente, que devemos começar de algum lugar.

\section{§1. O sistema formal $\mathrm{AcM}(\mathrm{V})$}

Comecemos pelo sistema mais fraco que iremos definir nesse estudo.Esse sistema será baseado nas cláusulas de acumulação positiva ou CAPs [cf., p. 37] e não permitirá que o princípio de indução seja aplicado às fórmulas nas quais o predicado de verdade aparece.

1.1. Definição. $\operatorname{AcM}(V)$ é o sistema cujos axiomas específicos são os axiomas de P.A. para $\mathrm{L}_{\text {P.A. }}$ [cf. p. 37] e as sentenças $\forall x\left(\mathrm{~V}_{n}(x) \rightarrow \mathrm{V}(x)\right)$, para todo $n$. 
$\operatorname{AcM}(\mathrm{V})$ é chamado sistema minimal (restrito) da acumulação positiva; onde 'acumulação positiva' se refere, obviamente, às CAPs, 'minimal' ao fato de que tãosomente tais cláusulas são “acrescentadas” à aritmética de primeira-ordem e ‘restrito' à restrição imposta ao esquema de indução matemática.

$\operatorname{AcM}(\mathrm{V})$ se opõe, por exemplo, ao sistema minimal de acumulação negativa, $\operatorname{AcM}(\mathrm{F})$, e ao sistema minimal não-restrito de acumulação positiva, AcMV .

Uma vez que o conjunto das DPVs (e, portanto, das CAPs) é decidível:

1.2. Teorema. AcM(V) é um sistema formal (no sentido hilbertiano [cf. p. 18]).

Notemos, então, que $\left\{i \mid i=g\left(\mathrm{~V}_{n}(x)\right)\right.$ para algum $\left.n\right\}$, ou seja, o conjunto dos números de Gödel das DPVs é decidível; embora, como consequência do teorema da indefinibilidade de Tarski, $\left\{i \mid \mathbb{N} \vDash \mathrm{V}_{n}(\bar{i})\right.$ para algum $\left.n\right\}(=\hat{\mathrm{V}})$ ou, em outras palavras, o conjunto dos números de Gödel das verdades da aritmética seja indecidível (de fato, $\hat{\mathrm{V}}$ não é nem mesmo um conjunto aritmético).

Nesse caso, a decidibilidade de $\left\{i \mid i=g\left(\mathrm{~V}_{n}(x)\right)\right.$ para algum $\left.n\right\}$ talvez possa emprestar certo aroma paradoxal à indefinibilidade da verdade; iremos, rapidamente, dissipá-lo.

Poderíamos, por exemplo, pensar que, por meio da contrapartida formal do conjunto de definições parciais, seríamos capazes de descrever uma contrapartida do conjunto das próprias verdades aritméticas; contudo, isso não é, de forma alguma, exato. Para que possamos vê-lo, devemos tomar DPV $(x)$ como uma contrapartida formal de $\left\{i \mid i=g\left(\mathrm{~V}_{n}(x)\right)\right.$ para algum $\left.n\right\}$, de modo que $j \in\left\{i \mid i=g\left(\mathrm{~V}_{n}(x)\right)\right.$, para algum $\left.n\right\}$ sse $\mathbb{N} \vDash \operatorname{DPV}(\bar{j})$. Ora, uma contrapartida formal da definição total da verdade seria, então, algo como: $\exists y(\operatorname{DPV}(y) \wedge \varphi(x, y))$. Entretanto, não avançamos aqui sequer um passo, uma vez que é uma das consequências do teorema da indefinibilidade de Tarski que não existe tal $\varphi(x, y)$ [cf. pp. 36-37]. 
Nossos primeiros resultados relativos ao sistema $\mathrm{AcM}(\mathrm{V})$ serão baseados em uma transformação (definida mais abaixo) das derivações de $\mathrm{AcM}(\mathrm{V})$ em derivações de P.A. (no caso, assumiremos que as únicas regras de inferência da lógica subjacente são o modus ponens e a generalização universal).

Seja uma derivação $\alpha=\left\langle\alpha_{1}, \alpha_{2}, \ldots, \alpha_{j}\right\rangle$ em AcM(V) tal que $\mathrm{V}_{i}$ é a DPV de maior complexidade em $\alpha$; seja, além disso, $[\beta] \mathrm{V} / \mathrm{V}_{i}$ uma substituição das ocorrências de $\mathrm{V}$ por $\mathrm{V}_{i}$ em $\beta$. Por exemplo, $[\mathrm{V}(\lceil\overline{1}=\overline{1}\rceil) \wedge \overline{2}=\overline{2}]^{\mathrm{V} / \mathrm{V}_{2}}$ será $\mathrm{V}_{2}(\lceil\overline{1}=\overline{1}\rceil) \wedge \overline{2}=\overline{2}$.

Definiremos, primeiramente, $/ \alpha /(1)$ :

a) $/ \alpha /(1)=\left\langle\left[\alpha_{1}\right] \mathrm{V} / \mathrm{V}_{i}\right\rangle$ se $\alpha_{1}$ é um axioma de P.A.;

b) $/ \alpha /(1)=\beta$, onde $\beta=\left\langle\beta_{1}, \beta_{2}, \ldots,\left[\alpha_{1}\right]^{\mathrm{V} / \mathrm{V}_{i}}\right\rangle$ é uma derivação de $\forall x\left(\mathrm{~V}_{k}(x) \rightarrow \mathrm{V}_{i}(x)\right)$ em P.A., se $\alpha_{1}$ é $\forall x\left(\mathrm{~V}_{k}(x) \rightarrow \mathrm{V}(x)\right)$, ou seja, se $\alpha_{1}$ é uma CAP.

Notemos que:

1) Se $\alpha_{1}$ é um axioma da lógica, então $\left[\alpha_{1}\right] \mathrm{V} / \mathrm{V}_{i}$ é um axioma da lógica e $\left\langle\left[\alpha_{1}\right]{ }^{\mathrm{V}} / \mathrm{V}_{i}\right\rangle$ é uma derivação em P.A..

2) Se $\alpha_{1}$ é um axioma de P.A., então $\left[\alpha_{1}\right]{ }^{\mathrm{V}} / \mathrm{V}_{i}$ é $\alpha_{1}$ e $\left\langle\left[\alpha_{1}\right] \mathrm{V}_{\mathrm{V}_{i}}\right\rangle$ é uma derivação em P.A..

3) Finalmente, se $\alpha_{1}$ é $\forall x\left(\mathrm{~V}_{k}(x) \rightarrow \mathrm{V}(x)\right)$, então, por IV-6.1 [cf. p. 96], existe uma derivação $\left\langle\beta_{1}, \beta_{2}, \ldots,\left[\alpha_{1}\right] \mathrm{V} / \mathrm{V}_{i}\right\rangle$ em P.A. (e, de fato, poderíamos efetivamente construir essa derivação).

Definiremos, agora, $/ \alpha /(n+1)$ :

a) $/ \alpha /(n+1)=$ a concatenação entre $/ \alpha /(n)$ e $\left\langle\left[\alpha_{n+1}\right] \mathrm{V}_{\mathrm{V}_{i}}\right\rangle$ se $\alpha_{n+1}$ é um axioma de P.A. ou se $\alpha_{n+1}$ é resultado da aplicação do modus ponens ou se $\alpha_{n+1}$ é resultado da aplicação da generalização universal.

b) $/ \alpha /(n+1)=$ a concatenação entre $/ \alpha /(n)$ e $\beta$, onde $\beta=\left\langle\beta_{1}, \beta_{2}, \ldots,\left[\alpha_{3}\right]{ }^{\mathrm{V}} / \mathrm{V}_{i}\right\rangle$ é uma derivação de $\forall x\left(\mathrm{~V}_{k}(x) \rightarrow \mathrm{V}_{i}(x)\right)$ em P.A., se $\alpha_{n+1}$ é $\forall x\left(\mathrm{~V}_{k}(x) \rightarrow \mathrm{V}(x)\right)$. 
Notemos que:

4) Se $\alpha_{2}$ é uma generalização universal de $\alpha_{1}$ e $\left[\alpha_{1}\right] \mathrm{V}_{\mathrm{V}}$ é um elemento da derivação $\beta$ em P.A., então a concatenação entre $\beta$ e $\left\langle\left[\alpha_{2}\right] \mathrm{V} / \mathrm{V}_{i}\right\rangle$ é uma derivação de $\left[\alpha_{2}\right] \mathrm{V} / \mathrm{V}_{i}$ em P.A..

5) Se $\alpha_{3}$ é resultado da aplicação de modus ponens às fórmulas $\alpha_{1}$ e $\alpha_{2}$, e, além disso, $\left[\alpha_{1}\right] \mathrm{V} / \mathrm{V}_{i}$ e $\left[\alpha_{2}\right] \mathrm{V} / \mathrm{V}_{i}$ são elementos da derivação $\beta$ em P.A.; então, a concatenação entre $\beta$ e $\left\langle\left[\alpha_{3}\right]^{\mathrm{V} / \mathrm{V}_{i}}\right\rangle$ é uma derivação de $\left[\alpha_{3}\right]^{\mathrm{V} / \mathrm{V}_{i}}$ em P.A..

1.3. Definição. Seja uma derivação $\alpha$ tal como descrita acima; tomaremos, então, $/ \alpha / \operatorname{como} / \alpha /(j)$.

1.4. Lema. Se $\alpha$ é uma derivação de $\alpha_{j} \operatorname{em~} \operatorname{AcM}(V)$ tal que $V_{i}$ é a DPV de maior complexidade de $\alpha$, então / $\alpha /$ será uma derivação de $\left[\alpha_{j}\right] \mathrm{V} / \mathrm{V}_{i}$ em P.A.. ${ }^{(1)}$

Uma vez que $[\beta]^{V} / V_{i}$ não afeta as fórmulas de $L_{P . A .}$, segue-se de 1.4 que:

1.5. Teorema. AcM(V) é conservativo sobre P.A..

E, portanto, que:

1.6. Corolário. Se P.A. é consistente, então $\mathrm{AcM}(\mathrm{V})$ é consistente.

Na verdade, no caso particular das CAPs, temos algo mais forte.

Seja AcP o sistema cujos únicos axiomas específicos são nossas CAPs, por meio da transformação definida em 1.3, temos que:

1.7. Teorema. AcP é conservativo sobre $\mathrm{CP}^{=}$(a teoria da identidade em $\left.L_{P . A .}\right)$.

Como exemplo de um resultado "positivo", de IV-3.4, [cf. p. 85], de IV-3.6 [cf. p. 88] e das CAPs, temos que:

1.8. Teorema. $\mathrm{AcM}(\mathrm{V}) \vdash \forall \tau(\mathrm{V}(\tau \doteq \tau)) \wedge \forall \tau(\mathrm{V}(\tau \dot{\leqslant} \tau))$.

1. Toda nossa argumentação é inspirada em Tarski [cf. Teorema III, 1956, pp. 256-257] e é empregada por Halbach no estabelecimento da conservatividade (e, consequentemente, da consistência) de vários dos sistemas estudados em Axiomatic Theories of Truth, e. g., Teorema 7.5 [Halbach, 2011, p. 55]. 
Segue-se de 1.8 e do Teorema 7.6 de Axiomatic Theories of Truth [Halbach, 2011, p. 57] que existe pelo menos um teorema de $\mathrm{AcM}(\mathrm{V})$ que não é teorema de $\mathrm{BT}(\mathrm{V})$ [cf. p. 31] (esse sistema é denominado TB, Tarski Biconditionals, por Halbach).

Além disso, segundo 1.7, diferentemente dos bicondicionais de Tarski [cf. ibidem, p. 55], as CAPs são conservativas sobre $\mathrm{CP}=.{ }^{(2)}$ Ou seja, em um sentido, essas últimas são mais fortes que os bicondicionais de Tarski, uma vez que existem teoremas de $\operatorname{AcM}(V)$ que não são teoremas de BT(V), em outro, são mais fracas, uma vez que são conservativas sobre $\mathrm{CP}^{=}$. Podemos, entretanto, definir uma versão, por assim dizer, parametrizada de $\mathrm{BT}(\mathrm{V})$, na qual $\forall \tau(\mathrm{V}(\tau \doteq \tau))$ é derivável [ibidem, p. 58]; o que sugere, primeiramente, uma comparação entre $\mathrm{AcM}(\mathrm{V})$ e essa versão de $\mathrm{BT}(\mathrm{V})$.

O sistema BUT(V) será uma versão parametrizada de BT(V) na qual introduziremos novos axiomas por meio do esquema: $\forall \tau\left(\operatorname{V}\left((\dot{\alpha})^{x} / \tau\right) \leftrightarrow \alpha(\operatorname{LTF}(\tau))\right)$ para $\alpha$ em $L_{P . A}$, cuja descrição pormenorizada será apresentada abaixo [cf. o sistema UTB de Halbach, ibidem, pp. 53-54]. Seja, por exemplo, $\varphi$ uma fórmula de $L_{\text {P.A. }}$ cuja única variável livre é $x$ e $g(\varphi)=n$. Ora, $g(x)=30 \mathrm{e}(z)^{x} / y$ é pensado como um termo com as três variáveis livres indicadas (uma variante notacional de $x(y / z)$ [cf. p. 25 e p. 69]).

Teríamos, então, a seguinte aplicação do esquema esboçado acima:

$$
\operatorname{but}(\varphi)=_{d f} \forall y\left(\operatorname{TF}(y) \rightarrow\left(\mathrm{V}\left((\bar{n})^{30} / y\right) \leftrightarrow \varphi(\operatorname{LTF}(y))\right)\right) .
$$

Dissemos, anteriormente, que BT(V) e $\mathrm{AcM}(\mathrm{V})$ são, em certo sentido, incomparáveis. Introduziremos, agora, dois sistemas, $\mathrm{CT}(\mathrm{V})$ e CUT(V), mais fracos e análogos, respectivamente, aos sistemas $\mathrm{BT}(\mathrm{V})$ e $\mathrm{BUT}(\mathrm{V})$, que permitirão avançarmos um pouco mais nas comparações com $\mathrm{AcM}(\mathrm{V})$.

2. De fato, seja BTP o sistema cujos axiomas específicos são os bicondicionais de Tarski; BTP demonstrará, por exemplo, a existência de pelo menos dois objetos, um "verdadeiro" e outro "falso". Grosso modo, o argumento é o seguinte: pela lógica, temos que (1) BTP $\vdash \overline{0}=\overline{0} \rightarrow \overline{0}=\overline{0}$ e que (2) BTP $\vdash \sim \sim(\overline{0}=\overline{0} \rightarrow \overline{0}=\overline{0})$; pelos bicondicionais de Tarski, (3) BTP $\vdash \mathrm{V}([\overline{0}=\overline{0} \rightarrow \overline{0}=\overline{0}\rceil)$ e (4) BTP $\vdash \sim \mathrm{V}(\lceil\sim(\overline{0}=\overline{0} \rightarrow \overline{0}=\overline{0})\rceil)$; portanto, temos que (5) $\mathrm{BTP} \vdash \exists x \mathrm{~V}(x)$ e que (6) BTP $\vdash \exists x \sim \mathrm{V}(x)$; e, finalmente, que (7) BTP $\vdash \exists x \exists y(\sim x=y)$ [cf. Halbach, 2011, p. 55]. 


\subsection{Definições de condicionais e bicondicionais tarskianos.}

a) A sentença $\alpha \rightarrow \mathrm{V}(\lceil\alpha\rceil)$ é chamada condicional de Tarski de $\alpha$;

b) Enquanto, $\alpha \leftrightarrow \mathrm{V}(\lceil\alpha\rceil)$ é chamada bicondicional de Tarski de $\alpha$ [cf. p. 24];

c) A sentença $\forall y\left(\operatorname{TF}(y) \wedge \alpha(\operatorname{LTF}(y)) \rightarrow \mathrm{V}\left((\dot{\alpha})^{30} / y\right)\right)$ ou, abreviadamente, att( $\left.\alpha\right)$ é chamada condicional uniforme de Tarski de $\alpha$;

d) Enquanto, $\forall y\left(\operatorname{TF}(y) \rightarrow\left(\alpha(\operatorname{LTF}(y)) \leftrightarrow \mathrm{V}\left((\dot{\alpha})^{\overline{30}} / y\right)\right)\right)$ ou, abreviadamente, $\operatorname{but}(\alpha)$ é chamada bicondicional uniforme de Tarski de $\alpha$.

Aos tipos de condicionais tarskianos correspondem, portanto, certas teorias, a saber: aos bicondicionais de Tarski, BT(V) [cf. p. 31]; aos bicondicionais uniformes, BUT(V). Defineremos, agora, os sistemas CT(V) e CUT(V) correspondentes, respectivamente, aos condicionais e aos condicionais uniformes de Tarski.

\subsection{Definição.}

a) $\mathrm{CT}(\mathrm{V})$ é o sistema cujos axiomas são os axiomas de P.A. para $L_{P . A .}$ e os condicionais tarskianos das fórmulas de $L_{P . A}$;

b) CUT(V) é o sistema cujos axiomas são os axiomas de P.A. para $L_{\text {P.A. }}$ e os condicionais tarskianos uniformes das fórmulas de $L_{\text {P.A. }}$.

Temos, claramente, que:

1.11. Teorema. $\mathrm{CT}(\mathrm{V})$ é um subsistema de $\mathrm{AcM}(\mathrm{V})$ [notemos que, pelo teorema 1.8, $\mathrm{AcM}(\mathrm{V})$ não é um subsistema de $\mathrm{CT}(\mathrm{V})]$

A próxima questão naturalmente seria: $\mathrm{CUT}(\mathrm{V})$ é um subsistema de $\mathrm{AcM}(\mathrm{V})$ ?

Contudo, antes de continuarmos, analisemos, visando uma apreensão mais intuitiva do sistema CUT(V), um caso particular, o axioma $\operatorname{att}(\exists z(x+z=x))$ ou, mais detalhadamente, $\forall y\left(\operatorname{TF}(y) \wedge \exists z(\operatorname{LTF}(y)+z=\operatorname{LTF}(y)) \rightarrow \mathrm{V}\left((\lceil\exists z(x+z=x)\rceil)^{\overline{30}} / y\right)\right)$. 
Notemos, primeiro, que tanto $\operatorname{TF}(\lceil\overline{2} \cdot \overline{3}\rceil) \wedge \exists z(\operatorname{LTF}(\lceil\overline{2} \cdot \overline{3}\rceil)+z=\operatorname{LTF}(\lceil\overline{2} \cdot \overline{3}\rceil))$ $\rightarrow \mathrm{V}\left((\lceil\exists z(x+z=x)\rceil)^{30} /\lceil\overline{2} \cdot \overline{3}\rceil\right)$, uma das instâncias de ât $(\exists z(x+z=x))$, quanto, de fato, todas as instâncias dos condicionais uniformes são deriváveis em $\mathrm{AcM}(\mathrm{V})$; o que mostra que tais condicionais são "corretos" em relação ao sistema $\mathrm{AcM}(\mathrm{V})$. Esse é o primeiro ponto de contato entre CUT(V) e AcM(V).

Temos, além disso, que $\mathrm{AcM}(\mathrm{V})+\mathrm{V}(\lceil\exists z((\overline{2} \cdot \overline{3})+z=(\overline{2} \cdot \overline{3}))\rceil)$ é uma consequência de P.A. $\vdash \operatorname{TF}(\lceil\overline{2} \cdot \overline{3}\rceil) \wedge \operatorname{LTF}(\lceil\overline{2} \cdot \overline{3}\rceil) \simeq \overline{6} \wedge \overline{2} \cdot \overline{3}=\overline{6} \wedge \exists z(\overline{6}+z=\overline{6})$ e das cláusulas de acumulação positivas. $\mathrm{E}$, embora $\mathrm{AcM}(\mathrm{V})+\mathrm{V}(\lceil\exists z((\overline{2} \cdot \overline{3})+z=(\overline{2} \cdot \overline{3}))\rceil)$ seja também uma consequência de P.A. $\vdash \forall x(x+\overline{0}=x)$, a ideia subjacente aos condicionais uniformes é que possamos fazer derivações análogas àquela primeira em $\operatorname{AcM}(V)$. Esse é nosso segundo ponto de contato entre os sistemas.

Infelizmente, como sabemos da discussão de IV §3 [pp. 85-86], a quantificação ' $\forall y$ ' no início de $\operatorname{att}(\exists z(x+z=x))$, pode mudar muito as coisas. Uma dose de "engenharia reversa" aplicada aos condicionais uniformes de Tarski, por exemplo, traria consigo uma série de princípios "filigranáticos" (mas, é claro, nem por isso em si mesmos desinteressantes): alguns até razoavelmente simples, como $\forall y \exists x(\operatorname{LTF}(x) \simeq y)$; outros complexos, como $\left.\left.\forall x \forall y \forall z\left(\operatorname{TF}(x) \wedge \mathrm{TF}(y) \wedge \operatorname{LTF}(x) \simeq \operatorname{LTF}(y) \wedge \mathrm{V}_{n}\left((z)^{\overline{30}} / x\right)\right) \rightarrow \mathrm{V}_{n}\left((z)^{\overline{30}} / y\right)\right)\right)$; esse último estabelecendo que se dois termos designam um mesmo número, eles são intersubstituíveis salva veritate.

De modo que, apesar dos pontos de contanto entre CUT(V) e AcM(V), a complexidade inerente desses últimos princípios coloca sérias dificuldades técnicas ao avanço de nossa comparação entre CUT(V) e AcM(V).

Deixemos claro, não estamos afirmando que possamos derivar os condicionais uniformes de Tarski dos princípios acima, nem que esses condicionais sejam deriváveis em $\mathrm{AcM}(\mathrm{V})$, nem que os próprios princípios acima sejam deriváveis em $\mathrm{AcM}(\mathrm{V})$; embora, nesse último caso, isso pareça, de fato, acontecer. 
Estamos afirmando que, analisando possíveis derivações dos condicionais, chegamos a uma aritmética "em filigrana" das relações entre as contrapatidas formais de valorações, termos e definições parciais de verdade que foi apenas esboçada anteriormente e cujos princípios mais básicos estariam entre, por exemplo, $\mathrm{I} \Sigma_{1} \vdash \forall \tau\left(\mathrm{V}_{0}(\tau \doteq \tau)\right)$ [IV-3.4, p. 84], I $\Sigma_{1} \vdash \forall n \neq m\left(\sim \mathrm{V}_{0}(\bar{n} \doteq \bar{m})\right)$ [IV-3.9, p. 89], e os princípios da suficiência da decomposição e da unicidade da leitura [pp. 70-71].

Deixaremos, portanto, uma primeira questão:

1.12. Questão. CUT(V) é um subsistema de $\operatorname{AcM}(V)$ ?

Como seria natural esperar, temos, agora, a questão conversa:

1.13. Questão. AcM(V) é um subsistema de CUT(V)?

Paralelamente ao caso anterior, em CUT(V) podemos derivar todas as instâncias das CAPs, o que não é, entretanto, subsídio suficiente, como já deve estar óbvio, para responder a questão acima. Notemos que, como no caso de $\operatorname{AcM}(V)$ quantificamos sobre "fórmulas" e no caso de CUT(V) quantificamos sobre "termos", uma comparação entre os sistemas não seria imediata.

Entretanto, mesmo deixando as duas questões em aberto, devemos notar que existe um certo parentesco entre os sistemas AcM(V) e CUT(V); de fato, daremos noutra ocasião uma definição mais rigorosa desse "parentesco".

Devemos, por um momento, voltar nossa atenção mais pontualmente para o sistema $\operatorname{AcM}(\mathrm{V})$.

Tipicamente, as cláusulas de acumulação de $\mathrm{AcM}(\mathrm{V})$ permitirão que, dadas certas condições metateóricas específicas, possamos estabelecer esquemas de teoremas em $\operatorname{AcM}(\mathrm{V})$. 
Por exemplo, podemos estabelecer que:

\subsection{Teoremas.}

a) Para qualquer sentença $\alpha$ de $\mathrm{L}_{\text {P.A. }}, \mathrm{AcM}(\mathrm{V})+\mathrm{V}(\lceil\alpha \rightarrow \alpha\rceil)$;

b) $E$, para qualquer sentença $\alpha$, se P.A. $\vdash \alpha$, então $A c M(V) \vdash V(\lceil\alpha\rceil)$.

Notemos que 1.14.b), por exemplo, é diferente do mais que trivial "se P.A. $\vdash \alpha$, então $\operatorname{AcM}(V)+\alpha "$.

O argumento para 1.14.b) é o seguinte: 1) Se $\alpha$ é uma sentença de $L_{\text {P.A. }}$, então $\alpha$ possui uma complexidade dada, digamos, $n$. 2) Portanto, por IV-6.3 [p. 97], temos que P.A. $\vdash \alpha \rightarrow \mathrm{V}_{n}(\lceil\alpha\rceil)$. E, assim, P.A. $\vdash \mathrm{V}_{n}(\lceil\alpha\rceil)$ e $\mathrm{AcM}(\mathrm{V}) \vdash \mathrm{V}(\lceil\alpha\rceil)$.

Desse modo, podemos estabelecer alguns enunciados de caratér geral em $\mathrm{AcM}(\mathrm{V})$, e. g., 1.14.a); entretanto, é discutível se outros enunciados com, por assim dizer, o mesmo conteúdo cognitivo não poderiam ser estabelecidos sem o auxílio do predicado $\mathrm{V}(x)$ de verdade [cf. discussão, p. 83]. Afinal, qual poderia ser a vantagem de 'para qualquer sentença $\alpha$ de $L_{\text {P.A. }}, \mathrm{AcM}(\mathrm{V}) \vdash \mathrm{V}([\alpha \rightarrow \alpha\rceil)$ ' em relação, por exemplo, a 'para qualquer sentença $\alpha$ de $L_{\text {P.A. }}$, P.A. $\vdash \alpha \rightarrow \alpha$ '?

Não obstante, vários outros resultados interessantes de caráter geral podem ser estabelecidos em $\operatorname{AcM}(\mathrm{V})$; por exemplo, uma forma fraca da correção da lógica subjacente:

1.15. Teorema. Seja $\varphi(x)$ uma fórmula de $L_{P . A}$, onde $\varphi(x)$ é pensada como a descrição de um conjunto de axiomas; seja $\operatorname{Pr}_{\varphi}(x)$ uma contrapartida formal (construída canonicamente) de " $x$ é derivável a partir dos axiomas de $|\varphi(x)|$ "; seja $\alpha$ uma sentença qualquer de $\mathrm{L}_{\text {P.A. }}$. Então, $\operatorname{AcM}(\mathrm{V}) \vdash\left(\forall x(\varphi(x) \rightarrow \mathrm{V}(x)) \wedge \operatorname{Pr}_{\varphi}(\lceil\alpha\rceil)\right) \rightarrow \mathrm{V}(\lceil\alpha\rceil)$.

Esses resultados não querem dizer que $\mathrm{AcM}(\mathrm{V})$ seja um sistema dedutivamente forte e deveremos investigar essa questão um pouco mais adiante. Antes disso, contudo, 
estudaremos rapidamente alguns dos modelos de $\mathrm{AcM}(\mathrm{V})$, notadamente, aqueles que são extensões do modelo padrão da aritmética.

\section{A. Extensões do modelo padrão para $\operatorname{AcM}(\mathrm{V})$}

1.16. Definição. No modelo padrão $\mathbb{N}(\hat{V})$ de $A c M(V)$ interpretaremos as constantes aritméticas como em $\mathbb{N}$ e $\mathrm{V}$ como $|\hat{V}|$, ou seja, como $\| x$ é verdadeira em $\mathbb{N} \|$ ou, em outras palavras, como $\{g(\alpha) \mid \mathbb{N} \vDash \alpha\}$.

1.17. Teorema. $\mathbb{N}(\hat{V})$ é realmente um modelo de $\operatorname{AcM}(V)$.

1.18. Definição. Um sistema S é (positivamente) auto-referencial se existe uma fórmula $\alpha$ de $\mathrm{L}_{\text {P.A.V. }}$ tal que $\alpha \notin \mathrm{L}_{\text {P.A. }}$ e $\mathrm{S} \vdash \mathrm{V}(\lceil\alpha\rceil) .{ }^{(3)}$

Segue-se, então, de 1.17 que:

1.19. Corolário. AcM(V) não é auto-referencial.

Obviamente, $\operatorname{AcM}(\mathrm{V})$ frequentemente se "pronuncia" sobre $\mathrm{V}$, por exemplo:

a) $\operatorname{AcM}(\mathrm{V})+\mathrm{V}(\lceil\mathrm{V}(\lceil\alpha\rceil)\rceil) \vee \sim \mathrm{V}(\lceil\mathrm{V}(\lceil\alpha\rceil)\rceil)$;

b) $\operatorname{AcM}(\mathrm{V})+\mathrm{V}(\lceil\lceil\mathrm{V}(\lceil\alpha\rceil)\rceil=\lceil\mathrm{V}(\lceil\alpha\rceil)\rceil\rceil)$.

É bastante simples compreender que não existe nenhuma contradição entre a) e 1.19. O segundo caso é um pouquinho mais tricky: devemos notar que, embora a fórmula $\mathrm{V}(\lceil\alpha\rceil)$ de $\mathrm{L}_{\text {P.A.V. }}$ "ocorra" em $\lceil\mathrm{V}(\lceil\alpha\rceil)\rceil,\lceil\mathrm{V}(\lceil\alpha\rceil)\rceil$ é um numeral de $L_{\text {P.A. }}$ e, portanto, $\lceil\mathrm{V}(\lceil\alpha\rceil)\rceil=\lceil\mathrm{V}(\lceil\alpha\rceil)\rceil$ é uma sentença de $\mathrm{L}_{\mathrm{P} . \mathrm{A}}$. Poderíamos dizer, então, que $\mathrm{AcM}(\mathrm{V})$ se pronuncia apenas obliquamente a respeito de $\mathrm{V}$.

1.20. Definição. Na interpretação $\mathbb{N}(\omega)$ de LP.A.V. as constantes aritméticas serão interpretadas como em $\mathbb{N}$, enquanto $\mathrm{V}$ será o conjunto dos números naturais.

3. Uma discussão mais substancial sobre a distinção entre sistemas "typed" e "type-free", relacionada à ideia de auto-referência, pode ser encontrada em Halbach [2011, pp. 140-145]. 
1.21. Teorema. $\mathbb{N}(\omega)$ é um modelo de $A c M(V)$.

A teoria de $\mathbb{N}(\omega)$ pode ser chamada, seguindo sugestões, respectivamente, de Priest [2006b, p. 12] e de Field [2008, p. 143], trivialismo ou hiper-dialetismo. De modo que $\mathrm{AcM}(\mathrm{V})$ é compatível com o trivialismo (algo que de modo nenhum seria promissor para Field [ibidem]).

Notemos, entretanto, que $\mathbb{N}(\omega)$ não é ele mesmo trivial (no sentido em que toda sentença de $L_{\text {P.A.V. }}$ é verdadeira em $\mathbb{N}(\omega)$ ).

Logo, diferentemente de BT(V)-no qual temos "se $\mathrm{S} \vdash \mathrm{BT}(\mathrm{V})$ é consistente, então não existe fórmula $\varphi(x)$ de $L_{\text {P.A. }}$ tal que $\mathrm{S} \vdash \forall x(\varphi(x) \leftrightarrow \mathrm{V}(x)$ )" (o que é uma espécie de teorema da indefinibilidade da verdade) [cf. Halbach, 2011, p. 54]-temos que:

1.22. Corolário. Se P.A. é consistente, então $\operatorname{AcM}(\mathrm{V}) \cup\{\forall x(x=x \leftrightarrow \mathrm{V}(x))\}$ também será consistente.

1.23. Definição. Na interpretação $N(T . P . A) ~ d e. ~ L_{P . A . V .}$, as constantes aritméticas serão interpretadas como em $\mathbb{N}$, enquanto $V$ será interpretado como $\| x$ é um teorema de P.A. $\|$ ou, em outras palavras, $\{g(\alpha) \mid$ P.A. $\vdash \alpha\}$.

Nosso próximo resultado estabelece que, apesar de ser pensado como um sistema minimal, $\mathrm{AcM}(\mathrm{V})$ não é totalmente trivial. $E$, incidentalmente, chama nossa atenção para a força expressiva das fórmulas de P.A. em relação à posição que ocupam na hierarquia da aritmética; no caso abaixo, tanto entre as definições parciais de certa complexidade e outras de maior complexidade (cada uma delas com sua posição peculiar na hierarquia), quanto entre as próprias DPVs e o conceito recursivamente enumerável ou $\Sigma_{1}$ de " $x$ é um teorema de P.A.".

1.24. Teorema. $\mathbb{N}(T . P . A$.$) não é um modelo de A c M(V)$. 
Prova. Seja $\mathrm{R}_{\text {P.A. }}$ a sentença de Rosser de P.A. e $n$ a complexidade de $\mathrm{R}_{\text {P.A.: }}$. Temos, pelas condições tarskianas para $\mathrm{V}_{n}(x)$ e $\mathrm{V}_{n+1}(x)$ [cf. IV-6.4.a), p. 97], que

a) $\operatorname{AcM}(\mathrm{V}) \vdash \mathrm{V}_{n}\left(\left\lceil\mathrm{R}_{\text {P.A. }}\right\rceil\right) \vee \mathrm{V}_{n_{+1}}\left(\left\lceil\sim \mathrm{R}_{\text {P.A. }}\right\rceil\right)$ e, portanto, que

b) $\operatorname{AcM}(\mathrm{V}) \vdash \mathrm{V}\left(\left\lceil\mathrm{R}_{\mathrm{P} . \mathrm{A} .}\right\rceil\right) \vee \mathrm{V}\left(\left\lceil\sim \mathrm{R}_{\text {P.A. }}\right\rceil\right)$.

Suponhamos que $\mathbb{N}($ T.P.A.) seja um modelo de $\operatorname{AcM}(\mathrm{V})$; de modo que temos

c) $\mathbb{N}$ (T.P.A. $) \vDash \operatorname{Pr}_{\text {P.A. }}\left(\left\lceil R_{\text {P.A. }}\right\rceil\right) \vee \operatorname{Pr}_{\text {P.A. }}\left(\left\lceil\sim R_{\text {P.A. }}\right\rceil\right)$.

Contudo,

d) se $\mathbb{N}$ (T.P.A. $) \vDash \operatorname{Pr}_{\text {P.A. }}\left(\left\lceil\mathrm{R}_{\text {P.A. }}\right\rceil\right)$, P.A. $\vdash \mathrm{R}_{\text {P.A. }}$;

e) $\operatorname{se} \mathbb{N}$ (T.P.A.) $\operatorname{Pr}_{\text {P.A. }}\left(\left\lceil\sim R_{\text {P.A. }}\right\rceil\right)$, então P.A. $\vdash \sim R_{\text {P.A. }}$.

Nos dois casos, seguir-se-ia da versão de Rosser do teorema da incompletude que P.A.é inconsistente e, portanto,

f) $\mathbb{N}(T . P . A$.$) não poderia ser, de qualquer modo, modelo de \mathrm{AcM}(\mathrm{V})$.

Assim, em um certo sentido, não seria possível equacionar derivabilidade em P.A. e verdade em $\mathrm{AcM}(\mathrm{V})$. Além disso, devemos notar que, no caso de BT(V), um análogo do Teorema 1.24 seria uma consequência trivial do teorema citado no comentário introdutório ao Corolário 1.22; pois, se $\mathrm{S} \vdash \mathrm{BT}(\mathrm{V})$ é consistente, então não existe fórmula $\varphi(x)$ de $\mathrm{L}_{\text {P.A. }}$ tal que $\mathrm{S} \vdash \forall x(\varphi(x) \leftrightarrow \mathrm{V}(x))$, de modo que $\operatorname{Pr}_{\text {P.A. }}(x)$ não poderia ser essa fórmula.

Quanto à interpretação de $\mathrm{V}$, temos, mais geralmente, que:

1.25. Definição. Na interpretação $\mathbb{N}(A)$ de $L_{\text {P.A.V., }}$ as constantes aritméticas serão interpretadas como em $\mathbb{N}$, enquanto V será interpretado como A.

Em suma, os resultados anteriores estabelecem que:

a) Se $\mathrm{N}(\mathrm{A})$ é um modelo de $\operatorname{AcM}(\mathrm{V})$, então $\{g(\alpha) \mid$ P.A. $\vdash \alpha\} \subseteq \mathrm{A}$ [1.14.b) , p. 108];

b) $\mathbb{N}(T . P . A$.$) não é um modelo de \operatorname{AcM}(\mathrm{V})[1.24$, p. 110];

c) Entretanto, $\mathbb{N}(\omega)$ é um modelo de $\operatorname{AcM}(V)[1.21$, p. 110]; 
d) Além disso, para qualquer sentença $\alpha$ de $\mathrm{L}_{\text {P.A. }}, \mathrm{AcM}(\mathrm{V}) \vdash \mathrm{V}(\lceil\alpha\rceil) \vee \mathrm{V}(\lceil\sim \alpha\rceil)$.

Assim, pensando em certa "minimalidade" das possíveis interpretações de V, a pergunta que cabe é se quaisquer "extensões completas" de P.A. são modelos de $\operatorname{AcM}(V)$.

1.26. Definição. Um subconjunto A de números naturais é chamado - completo ou neg-completo se, para toda sentença $\alpha$ de $\mathrm{L}_{\text {P.A. }}, g(\alpha) \in \mathrm{A}$ ou $g(\sim \alpha) \in \mathrm{A}$.

Como uma espécie de caracterização de $\mathrm{AcM}(\mathrm{V})$ relativa (bem entendido, relativa) ao modelo padrão $\mathbb{N}$ da aritmética, temos:

1.27. Teorema. $\mathbb{N}(\mathrm{A})$ é um modelo de $\mathrm{AcM}(\mathrm{V})$ se e somente se A é -completo e $\{g(\alpha) \mid$ P.A. $\vdash \alpha\} \subseteq$ A

Anteriormente, foi ventilado um certo "parentesco" entre $\mathrm{AcM}(\mathrm{V})$ e CUT(V) [cf. p. 106]; de fato, temos que:

\subsection{Teoremas.}

a) $\mathbb{N}(\omega)$ é um modelo de CUT(V) [cp. 1.21, p. 110];

b) $\mathbb{N}$ (T.P.A.) não é um modelo de CUT(V) [cp. 1.24, p. 110];

c) E, geralmente, $\mathbb{N}(\mathrm{A})$ é um modelo de CUT(V) se e somente se A é -completo e $\{g(\alpha) \mid$ P.A. $\vdash \alpha\} \subseteq$ A [cp. 1.27].

E, além disso, um argumento por indução na complexidade das fórmulas de $L_{P . A}$. (apesar das considerações anteriores [cf. pp. 106-107]) parece nos conduzir a:

1.29. Conjectura. CUT(V) é um subsistema de $A c M(V)$.

Como pudemos notar-e isso não é nada surpreendente- $\mathrm{AcM}(\mathrm{V})$ aceita modelos dialéticos e mesmo triviais ou hiper-dialéticos [cf. p. 110], embora $\mathrm{AcM}(\mathrm{V})$ seja ele mesmo, na suposição de que P.A. o é, consistente. Essa é, na verdade, uma das 
características principais das cláusulas de acumulação e, possivelmente, uma de suas principais fraquezas: $\mathrm{AcM}(\mathrm{V})$ aceita, poderíamos argumentar, mais do que deveria.

Lembremos, entretanto, que $\mathrm{AcM}(\mathrm{V})$ é tomado nesse estudo mais como um exemplo e uma etapa da constituição de sistemas formais da verdade da aritmética, por meio de definições parciais de verdade, do que um sistema que devemos sustentar como aquele mais adequado. E, além disso, muitos filósofos com tendências deflacionistas argumentariam que esse tipo de fraqueza é, de fato, desejada.

Um pouco de argumentação desinteressada não deixa de ser bem-vinda (e mesmo esclarecedora): uma vez que, para sentenças de $\mathrm{L}_{\text {P.A. }}$, $\mathrm{AcM}(\mathrm{V}) \vdash \mathrm{V}(\lceil\alpha\rceil) \vee \mathrm{V}(\lceil\sim \alpha\rceil)$, $\mathrm{AcM}(\mathrm{V})$ é refratário ao indetermismo (em L.A.); em compensação, $\mathrm{AcM}(\mathrm{V})$ é compatível com o dialetismo (de fato, com o trivialismo).

Ora, talvez isso não seja o fim do mundo. Suponhamos que $\mathbb{M}$ seja um modelo de $\operatorname{AcM}(V)$ tal que $\mathbb{M} \vDash V(\lceil\alpha\rceil) \vee V(\lceil\sim \alpha\rceil)$ e que P.A. seja consistente. Segue-se, então, que existem verdades (em $\mathbb{M}$ ) que não são deriváveis em P.A., mas isso é simplesmente uma forma do teorema da incompletude e, portanto, não deveria espantar ninguém. Ao que parece, a consistência é uma condição necessária para nossos sistemas formais sejam úteis quando dados na lógica clássica, mas não é, presumivelmente, uma condição necessária para a verdade pensada como acumulação.

Já aqui, podemos notar um tipo de deflacionismo radical, cujas possibilidades auto-referenciais e estruturais que serão esboçadas no próximo capítulo, acabarão por tornar cada vez mais atraente.

Encerrando nossas considerações modelo-teoréticas, apresentaremos um resultado muito interessante para as relações entre $\mathrm{AcM}(\mathrm{V})$ e o "deflacionismo descitacionista" do mote "truth is disquotation". 
Segue-se de 1.27 que:

1.30. Lema. Se $\alpha$ é uma sentença de $L_{P . A .}$ mas não é um teorema de P.A., então existe uma interpretação $\mathbb{N}(\mathrm{A})$ de $L_{\text {P.A.V. }}$ tal que $\mathbb{N}(\mathrm{A}) \vDash \mathrm{V}(\lceil\alpha\rceil)$.

E, portanto, temos que:

1.31. Teorema da descitação. Se $\operatorname{AcM}(\mathrm{V}) \vdash \mathrm{V}(\lceil\alpha\rceil)$, então P.A. $\vdash \alpha$.

Obviamente, existem inúmeras questões sobre modelos $\mathbb{M}(\mathrm{A})$ de $\mathrm{AcM}(\mathrm{V})$, nos quais M é um modelo desviante (non-standard) de P.A.; entretanto não teremos ocasião de analisá-las nesse estudo.

Analisaremos, agora, as "fraquezas" de AcM(V) em outra chave.

\section{B. Alguns resultados da teoria da derivação}

1.32. Teorema. Se $\operatorname{AcM}(\mathrm{V}) \vdash \forall x(\varphi(x) \rightarrow \mathrm{V}(x))$, então deve existir $n$ tal que P.A. $\vdash \forall x\left(\varphi(x) \rightarrow \operatorname{SENT}_{n}(x)\right)$.

Prova. Seja $\alpha$ uma derivação de $\forall x(\varphi(x) \rightarrow \mathrm{V}(x))$ em $\operatorname{AcM}(\mathrm{V})$ tal que $\mathrm{V}_{i}$ é a DPV de maior complexidade que ocorre em $\alpha$. Transformamos $\alpha$ em / $\alpha /$ como no Lema 1.4 [cf. p. 103]; / $\alpha /$ será, então, uma derivação de $\forall x\left(\varphi(x) \rightarrow \mathrm{V}_{i}(x)\right)$ em P.A.. Assim, uma vez que P.A. $\vdash \forall x\left(\mathrm{~V}_{i}(x) \rightarrow \operatorname{SENT}_{i}(x)\right)$, temos que P.A. $\vdash \forall x\left(\varphi(x) \rightarrow \operatorname{SENT}_{i}(x)\right)$.

As consequências do teorema são, basicamente, que não podemos derivar em $\mathrm{AcM}(\mathrm{V})$ vários princípios gerais para os quais existem "versões metateóricas" da forma: "Se $\varphi$ é uma sentença de ..., então $\mathrm{AcM}(\mathrm{V}) \vdash \ldots \varphi$...".

1.33. Teoremas. Se P.A. é consistente,

a) $\operatorname{AcM}(\mathrm{V}) \Vdash \forall x(\operatorname{SENT}(x) \rightarrow \mathrm{V}(\dot{\rightarrow}(x, x)))$ [cp. 1.14.a), p. 108];

b) $\operatorname{AcM}(\mathrm{V}) \Vdash \forall x\left(\operatorname{Pr}_{\text {P.A. }}(x) \rightarrow \mathrm{V}(x)\right)$ [cp. 1.14.b), p. 108]. 


\section{As demandas tarskianas em $\mathrm{AcM}(\mathrm{V})$}

É consequência das considerações anteriores que quase todas as demandas tarskianas discutidas em \$1.3 e \$2.3 do capítulo I [cf. pp. 24-25 e pp. 34-36] não são satisfeitas pelo nosso sistema $\mathrm{AcM}(\mathrm{V})$.

A exceção óbvia é justamente uma forma fraca das CAPs que se verifica:

1.34. Teorema. Para qualquer sentença $\alpha$ de $L_{P . A .}, \operatorname{AcM}(V) \vdash \alpha \rightarrow V(\lceil\alpha\rceil)[c p$. Teorema I-a), p. 24].

O primeiro dos nossos resultados negativos será:

1.35. Teorema. Existe uma sentença $\alpha$ de $L_{\text {P.A. }}$ tal que $\operatorname{AcM}(\mathrm{V}) \Vdash \mathrm{V}(\lceil\alpha\rceil) \rightarrow \alpha$ [cp. Teorema I-a), p. 24].

Prova. Nosso modelo $\mathbb{N}(\omega)$ [cf. p. 109] e a sentença $R_{P . A .}$ de Rosser são suficientes para tanto.

a) Suponhamos, por absurdo, que $\mathrm{AcM}(\mathrm{V}) \vdash \mathrm{V}\left(\left\lceil\sim \mathrm{R}_{\text {P.A. }}\right\rceil\right) \rightarrow \sim \mathrm{R}_{\text {P.A: }}$. Segue-se que

b) $\mathrm{N}(\omega) \vDash \mathrm{V}\left(\left\lceil\sim \mathrm{R}_{\text {P.A. }}\right\rceil\right) \rightarrow \sim \mathrm{R}_{\text {P.A. }}$ e, desde que $\mathrm{N}(\omega) \vDash \mathrm{V}\left(\left\lceil\sim \mathrm{R}_{\text {P.A. }}\right\rceil\right)$,

c) $\mathbb{N}(\omega) \vDash \sim R_{\text {P.A. }}$, o que contradiz $\mathbb{N} \vDash R_{\text {P.A. }}$.

Usando novamente $\mathbb{N}(\omega)$, podemos estabelecer:

1.36. Teorema. $\operatorname{AcM}(\mathrm{V}) \Vdash \forall x(\mathrm{~V}(x) \rightarrow \operatorname{SENT}(x))$ [cp. Teorema I-b), p. 24].

Notemos que $\mathbb{N}(\omega) \vDash \mathrm{V}\left(\left\lceil\sim \mathrm{R}_{\text {P.A. }}\right\rceil\right) \wedge \mathrm{V}\left(\left\lceil\mathrm{R}_{\text {P.A. }}\right\rceil\right) \wedge \operatorname{SENT}\left(\left\lceil\mathrm{R}_{\text {P.A. }}\right\rceil\right) \wedge \dot{\sim}\left(\left\lceil\mathrm{R}_{\text {P.A. }}\right\rceil\right)=$ $\left\lceil\sim R_{\text {P.A. }}\right\rceil$, temos, então, que:

1.37. Teorema. $\operatorname{AcM}(\mathrm{V}) \Vdash \forall x(\operatorname{SENT}(x) \rightarrow(\sim \mathrm{V}(x) \vee \sim \mathrm{V}(\dot{\sim} x))))$. De fato, existe $\alpha$ tal que $\alpha$ é uma sentença de $L_{\text {P.A. }}$ e $\mathrm{AcM}(\mathrm{V}) \Vdash \sim \mathrm{V}(\lceil\alpha\rceil) \vee \sim \mathrm{V}(\lceil\sim \alpha\rceil)$ [cp. Teorema II, p. 24]. 
O próximo teorema exige um rápido mergulho nos modelos desviantes de P.A.:

1.38. Teorema. $\operatorname{AcM}(\mathrm{V}) \Vdash \forall x(\operatorname{SENT}(x) \rightarrow(\mathrm{V}(x) \vee \mathrm{V}(\dot{\sim} x)))$ [cp. Teorema III, p. 25].

Prova. Seja M um modelo desviante de P.A. e A é um subconjunto do domínio de M tal que:

a) o número de Gödel de nenhuma fórmula cuja complexidade lógica é "infinita" (no sentido em que nela existe um número “infinito” de constantes lógicas) esteja em A e tal que:

b) os números de Gödel de sentenças atômicas “infinitas” (ou seja, com algum termo de comprimento infinito) bem como as "consequências" finitas dessas sentenças (segundo nossas DPVs) estejam em A.

c) Uma vez que nossas CAPs são introduzidas por meio dos parâmetros metateóricos: 0, 1, 2, 3, etc., e nenhum número infinito de $\mathbb{M}$ aparece nessa lista; então, $\mathbb{M}(\mathrm{A})$ será um modelo de $\mathrm{AcM}(\mathrm{V})$.

Seja $\beta$, por exemplo, uma disjunção cujos disjuntos são todos $\overline{0}=\overline{0}$, cuja complexidade é dada por um número “infinito" de $\mathbb{M}$ e cujo número de Gödel em $\mathbb{M}$ é $\lambda$.

Temos, então, que

d) $\mathrm{M}(\mathrm{A}) \vDash \sim \mathrm{V}(\dot{\sim}(x)) \wedge \sim \mathrm{V}(x) \wedge \operatorname{SENT}(x)[\lambda]$, ou seja, a atribuição do número $\lambda$ à variável $x$ satisfaz $\sim \mathrm{V}(\dot{\sim}(x)) \wedge \sim \mathrm{V}(x) \wedge \operatorname{SENT}(x)$ em $\mathbb{M}(\mathrm{A})$.

$\mathrm{E}$, portanto,que $\operatorname{AcM}(\mathrm{V}) \Vdash \forall x(\operatorname{SENT}(x) \rightarrow(\mathrm{V}(x) \vee \mathrm{V}(\dot{\sim} x))))$.

Algo interessante é que temos uma forma fraca para a demanda tarskiana relativa ao Teorema III [cf. p. 25]: para qualquer sentença $\alpha$ de $L_{\text {P.A. }}, \mathrm{AcM}(\mathrm{V}) \vdash \mathrm{V}(\lceil\alpha\rceil) \vee \mathrm{V}(\lceil\sim \alpha\rceil)$. O que contrasta com o caso do Teorema II [cf. 1.37 p. 115].

Finalmente, já sabemos que $\operatorname{AcM}(\mathrm{V}) \Vdash \forall x\left(\operatorname{Pr}_{\mathrm{P} . \mathrm{A} .}(x) \rightarrow \mathrm{V}(x)\right)$ [1.33.b), p. 114] (cp. Teorema IV [cf. p. 25]). 
Argumentamos anteriormente que não há problema em aceitar instâncias do esquema de indução nas quais a constante $\mathrm{V}$ aparece [cf. p. 31]. No caso que estamos tratando agora, não há, pelo menos até esse ponto da pesquisa, nenhum problema em não aceitá-las; uma vez que os principais resultados apresentados nessa seção se aplicam também ao sistema formal AcMV [cf. p. 38], no qual a restrição do esquema de indução às fórmulas de $L_{P . A}$ é relaxada. Por exemplo, AcMV é conservativo sobre P.A. e AcMV $\forall \forall x\left(\operatorname{Pr}_{\text {P.A. }}(x) \rightarrow \mathrm{V}(x)\right)$.

\section{Halbach versus $\$ 1$}

Apesar da incompletude de nossa análise, cobrimos, por assim dizer, as bases do capítulo VII de Halbach [2011, pp. 53-62] e fomos um pouco além. Temos, grosso modo, análogos de todos teoremas de Halbach:

a) A indefinibilidade da verdade módulo $L_{P . A}$-fórmulas para extensões de BT(V) [cf. idem, p. 54] falha para $\operatorname{AcM}(\mathrm{V})$, uma vez que $\operatorname{AcM}(\mathrm{V}) \cup\{\forall x(x=x \leftrightarrow \mathrm{V}(x))\}$ é uma extensão de $\operatorname{AcM}(\mathrm{V})$ na qual a consistência é preservada [cf., 1.22, p. 110].

b) Diferentemente dos bicondicionais de Tarski [cf. Halbach, 2011, p. 55], as cláusulas de acumulação primitiva são conservativas sobre $\mathrm{CP}^{=}$[cf., 1.7, p. 103].

c) BT(V) e AcM(V) são conservativos sobre P.A. [Halbach, 2011, p. 54; nosso 1.5, p. 103, respectivamente].

d) Não é possivel demonstrar em BT(V) e AcM(V) "generalizações intra-sistêmicas" de leis metateóricas que envolvam todas as sentenças de $L_{P . A}$. [Halbach, 2011, p. 58; nosso 1.33, p. 114]. 


\section{§2. O Sistema Formal AcM(VF)}

Nessa seção, introduziremos, primeiramente, o sistema $\operatorname{AcM}(\mathrm{F})$, dual de $\mathrm{AcM}(\mathrm{V})$; depois, consideraremos algumas "hibridizações" desses sistemas. Para tanto, partiremos do sistema minimal da dupla acumulação, $\mathrm{AcM}(\mathrm{VF})$, ao qual acrescentaremos certos "axiomas de interação" para os predicados V e F.

2.1. Definição. $\operatorname{AcM}(\mathrm{F})$ é o sistema cujos axiomas são os axiomas de P.A. para $\mathrm{L}_{\text {P.A. }}$ e as sentenças $\forall x\left(\mathrm{~F}_{n}(x) \rightarrow \mathrm{F}(x)\right)$, para todo $n$.

$\operatorname{AcM}(\mathrm{F})$ é chamado sistema minimal (restrito) da acumulação negativa.

Alguns resultados análogos aos da seção anterior podem ser facilmente estabelecidos em ou para $\mathrm{AcM}(\mathrm{F})$.

\subsection{Teoremas.}

a) $\operatorname{AcM}(\mathrm{F})$ é um sistema formal;

b) $\operatorname{AcM}(\mathrm{F})$ é conservativo sobre P.A.;

c) Para qualquer sentença $\alpha$, se P.A. $\vdash \sim \alpha$, então $\operatorname{AcM}(\mathrm{F}) \vdash \mathrm{F}(\lceil\alpha\rceil)$;

d) $\operatorname{Se} \operatorname{AcM}(\mathrm{F}) \vdash \forall x(\varphi(x) \rightarrow \mathrm{F}(x))$, existe $n$ tal que P.A. $\vdash \forall x\left(\varphi(x) \rightarrow \operatorname{SENT}_{n}(x)\right)$.

Outros análogos da seção anterior demandam maior preparação e/ou reflexão.

\subsection{Definições.}

a) Escreveremos $\forall \tau(\mathrm{F}(\tau \doteq \mathrm{SUC}(\tau)))$ no lugar de $\forall x y(\mathrm{TF}(x) \wedge \dot{\doteq}(x, \operatorname{SUC}(x)) \approx y \rightarrow \mathrm{F}(y)) ;$

b) $\mathrm{E} \forall \tau(\mathrm{F}(\mathrm{SUC}(\tau) \dot{\leqslant} \tau))$ no lugar de $\forall x y(\mathrm{TF}(x) \wedge \dot{\leqslant}(\operatorname{SUC}(x), x) \approx y \rightarrow \mathrm{F}(y))$.

2.4. Teorema. $\operatorname{AcM}(\mathrm{F}) \vdash \forall \tau(\mathrm{F}(\tau \doteq \mathrm{SUC}(\tau))) \wedge \forall \tau(\mathrm{F}(\mathrm{SUC}(\tau) \dot{\leqslant} \tau))$.

2.5. Definição. No modelo padrão $\mathbb{N}(\hat{\mathrm{F}})$ de $\operatorname{AcM}(\mathrm{F})$ interpretaremos as constantes aritméticas como em $\mathbb{N}$ e F como $|\hat{\mathrm{F}}|$ ou, em outras palavras, $\{g(\alpha) \mid \mathbb{N} \vDash \sim \alpha\}$. 
2.6. Teorema. $\mathbb{N}(\hat{\mathrm{F}})$ é realmente um modelo de $\mathrm{AcM}(\mathrm{F})$.

2.7. Definição. Um sistema A é negativamente auto-referencial se existe uma fórmula $\alpha$ de $L_{\text {P.A.V. }}$ tal que $\alpha \notin L_{\text {P.A. }}$ e $A \vdash F(\lceil\alpha\rceil)$.

2.8. Corolário. $\mathrm{AcM}(\mathrm{F})$ não é negativamente auto-referencial.

2.9. Definição. $A c M(V F)$ é o sistema cujos axiomas são os axiomas de P.A. para $\mathrm{L}_{\text {P.A. }}$, e as sentenças $\forall x\left(\mathrm{~F}_{n}(x) \rightarrow \mathrm{F}(x)\right)$ e $\forall x\left(\mathrm{~V}_{n}(x) \rightarrow \mathrm{V}(x)\right)$, para todo $n$.

$\mathrm{AcM}(\mathrm{VF})$ é chamado sistema minimal (restrito) da dupla acumulação.

Não é difícil notar que métodos análogos aos da seção anterior são suficientes para estabelecer as principais propriedades de $\mathrm{AcM}(\mathrm{VF})$.

\subsection{Teoremas.}

a) $\mathrm{AcM}(\mathrm{VF})$ é conservativo sobre P.A.;

b) Para qualquer sentença $\alpha$ de $L_{\text {P.A., }}, \operatorname{AcM}(\mathrm{VF}) \vdash \mathrm{V}(\lceil\alpha\rceil) \vee \mathrm{F}(\lceil\alpha\rceil)$;

c) $\mathrm{Se} \mathrm{AcM}(\mathrm{VF}) \vdash \forall x(\varphi(x) \rightarrow \mathrm{F}(x))$ ou $\mathrm{AcM}(\mathrm{VF}) \vdash \forall x(\varphi(x) \rightarrow \mathrm{V}(x))$, então existe $n$ tal que P.A. $\vdash \forall x\left(\varphi(x) \rightarrow \operatorname{SENT}_{n}(x)\right)$.

Com um pouco mais de reflexão, podemos notar que os predicados de verdade e falsidade praticamente não interagem em $\mathrm{AcM}(\mathrm{VF})$; entretanto, segundo a estratégia que estamos adotando nesse estudo, isso não deve ser tomado como uma desvantagem dos nossos sistemas minimais em geral ou de $\mathrm{AcM}(\mathrm{VF})$ em particular.

De um ponto de vista, podemos argumentar que $\mathrm{AcM}(\mathrm{VF})$ é uma boa teoria exatamente por causa de seu caráter parcimonioso [cf. pp. 35-36 e p. 113]. De outro, $\operatorname{AcM}(\mathrm{VF})$ se torna um sistema adequado para o estudo da interação entre verdade e falsidade por meio da adição de novos axiomas [cf. p. 39]. 
Existem, pelo menos, dois príncipios óbvios e presumivelmente corretos de interação entre verdade e falsidade:

a) o princípio de determinação: uma sentença deve ser verdadeira ou falsa;

b) o princípio de separação: uma sentença não pode ser, dado um contexto determinado, verdadeira e falsa.

Em consonância com esses princípios, temos, então, que:

\subsection{Definições.}

a) A sentença $\mathrm{V}(\lceil\alpha\rceil) \vee \mathrm{F}(\lceil\alpha\rceil)$ será chamada a determinação de $\alpha$;

b) $\mathrm{e} \sim \mathrm{V}(\lceil\alpha\rceil) \vee \sim \mathrm{F}(\lceil\alpha\rceil)$ será a separação de $\alpha$;

c) $\forall x(\mathrm{~V}(x) \vee \mathrm{F}(x))$ será o axioma da determinação ou $A x D$;

d) e $\forall x(\sim \mathrm{V}(x) \vee \sim \mathrm{F}(x))$ será o axioma da separação ou $A x S$.

Notemos que, por 2.10.b), todas as determinações de sentenças de $L_{P . A}$ já são teoremas de $\mathrm{AcM}(\mathrm{VF})$; contudo, podemos pensar em vários "fortalecimentos" de $\mathrm{AcM}(\mathrm{VF})$, baseados nas fórmulas acima.

\subsection{Definições.}

a) $\operatorname{AcM}(\mathrm{VF})+E s q S$ é o sistema cujos axiomas são os axiomas de $\mathrm{AcM}(\mathrm{VF})$ e as sentenças $\sim \mathrm{V}([\alpha]) \vee \sim \mathrm{F}([\alpha])$, para toda sentença $\alpha$ de $\mathrm{L}_{\text {P.A. }}(\mathrm{AcM}(\mathrm{VF})+\mathrm{o}$ esquema de separação);

b) $\operatorname{AcM}(\mathrm{VF})+A x D$ é o sistema cujos axiomas são aqueles de $\mathrm{AcM}(\mathrm{VF})$ e $A x D$;

c) $\operatorname{AcM}(\mathrm{VF})+A x S$ é o sistema cujos axiomas são aqueles de $\mathrm{AcM}(\mathrm{VF})$ e $A x S$;

d) $\mathrm{E}$, finalmente, $\mathrm{AcM}(\mathrm{VF})+A x D+A x S$ é o sistema cujos axiomas são aqueles de $\operatorname{AcM}(\mathrm{VF}), A x D$ e $A x S$.

Novamente, usando métodos análogos aos da seção anterior [pp. 102-103], podemos estabelecer as principais propriedades de $\mathrm{AcM}(\mathrm{VF})+E s q S$. 


\subsection{Teoremas.}

a) $\operatorname{AcM}(\mathrm{VF})+E s q S$ é conservativo sobre P.A.;

b) Se AcM(VF) $+E s q S \vdash \forall x(\varphi(x) \rightarrow \mathrm{F}(x))$, então existe $n$ tal que P.A. $\vdash \forall x\left(\varphi(x) \rightarrow \operatorname{SENT}_{n}(x)\right)$;

c) Se AcM(VF) $+E s q S \vdash \forall x(\varphi(x) \rightarrow \mathrm{V}(x))$, então existe algum $n$ tal que P.A. $\vdash \forall x\left(\varphi(x) \rightarrow \operatorname{SENT}_{n}(x)\right)$.

Temos, além disso, nosso principal resultado comparativo com respeito ao sistema $\mathrm{AcM}(\mathrm{VF})+E s q S$ [cf. 1.11, p. 105]:

2.14. Teorema. BT(V) é um subsistema de $\mathrm{AcM}(\mathrm{VF})+E s q S$.

Prova. Para qualquer sentença $\alpha$ de $L_{\text {P.A. }}$, temos que:

a) $\operatorname{AcM}(V)+\alpha \rightarrow V(\lceil\alpha\rceil)$;

b) $\operatorname{AcM}(\mathrm{F}) \vdash \sim \alpha \rightarrow \mathrm{F}(\lceil\alpha\rceil)$;

c) $\operatorname{AcM}(\mathrm{VF})+E s q S \vdash \sim \mathrm{V}(\lceil\alpha\rceil) \vee \sim \mathrm{F}(\lceil\alpha\rceil)$;

d) $\mathrm{E}$, portanto, que $\mathrm{AcM}(\mathrm{VF})+E s q S \vdash \alpha \leftrightarrow \mathrm{V}(\lceil\alpha\rceil)$.

2.15. Corolário. AcM(VF) $+E s q S \vdash \sim \alpha \leftrightarrow \mathrm{F}(\lceil\alpha\rceil)$.

De modo que, se entendermos os bicondicionais de Tarski como pedra de toque do princípio de citação/descitação, podemos acrescentar, ao mote "verdade é descitação", que “citação/descitação é, essencialmente, acumulação + separação".

Não estudaremos os demais sistemas da Definição 2.12 (nem aprofundaremos nosso estudo de $\mathrm{AcM}(\mathrm{VF})+E s q S)$; optaremos por introduzir novos sistemas minimais, baseados em modificações das nossas DPVs (o que faremos no próximo capítulo).

Para encerrar, chamaremos atenção para o fato de que os métodos utilizados para estabelecer a conservatividade (em relação a P.A.) dos sistemas que estudamos até aqui não podem aplicados nos demais sistemas da Definição 2.12. 


\section{§3. O Sistema Formal AcS(V)}

Introduziremos, agora, um último sistema formal inspirado na ideia de acumulação de DPVs (não modificadas).

Uma variante óbvia dos sistemas apresentados anteriormente será o sistema $\operatorname{AcS}(\mathrm{V})$ definido abaixo. De fato, na figura de seus axiomas característicos, $\mathrm{AcS}(\mathrm{V})$ incorporará temas tanto "acumulativos" e quanto "descitacionais".

(Notemos que muitas de nossas considerações anteriores sobre AcM(V) [cf. 1.28 p. 112 e 1.31 p. 114], AcM(VF) [cf. 2.10 p. 119] e, principalmente, $\operatorname{AcM}(\mathrm{VF})+E s q S$ [cf. 2.13 e 2.14 p. 121] já aproximam esses temas.)

3.1. Definição. $\operatorname{AcS}(V)$ é o sistema cujos axiomas são os axiomas de P.A. para $\mathrm{L}_{\text {P.A. }}$ e as sentenças $\forall x\left(\operatorname{SENT}_{n}(x) \rightarrow\left(\mathrm{V}_{n}(x) \leftrightarrow \mathrm{V}(x)\right)\right)$, para todo $n$.

$\mathrm{AcS}(\mathrm{V})$ é chamado sistema (restrito) da acumulação simples.

Exatamente os mesmos métodos de \$1 estabelecem:

\subsection{Teoremas.}

a) $\mathrm{AcS}(\mathrm{V})$ é conservativo sobre P.A.;

b) $\mathrm{Se} \mathrm{AcS}(\mathrm{V}) \vdash \forall x(\varphi(x) \rightarrow \mathrm{V}(x))$, então existe $n$ tal que P.A. $\forall \forall x\left(\varphi(x) \rightarrow \operatorname{SENT}_{n}(x)\right)$.

É fácil notar que:

3.3. Teorema. $\operatorname{AcS}(\mathrm{V}) \vdash \forall \tau(\mathrm{V}(\tau \doteq \tau)) \wedge \forall \tau(\mathrm{V}(\tau \dot{\leqslant} \tau))$.

E, portanto, que:

4.4. Teorema. $\mathrm{AcS}(\mathrm{V})$ não é um subsistema de $\mathrm{BT}(\mathrm{V})$.

Enquanto que:

3.5. Teorema. BT(V) é um subsistema de $\mathrm{AcS}(\mathrm{V})$. 


\section{Prova.}

Para qualquer sentença $\alpha$ de $L_{\text {P.A. }}$ dada, cuja complexidade é, por exemplo, $n$, temos que:

a) P.A. $\vdash \operatorname{SENT}_{n}(\lceil\alpha\rceil) \wedge \mathrm{V}_{n}(\lceil\alpha\rceil) \leftrightarrow \alpha$;

b) $E$, portanto, que $\operatorname{AcS}(V)+\alpha \leftrightarrow V(\lceil\alpha\rceil)$.

Por motivos que já deveriam estar claros [cf. discussão pp. 106-107], a comparação entre $\mathrm{AcS}(\mathrm{V})$ e BUT(V) é bem mais complicada.

Em todo caso, $\mathrm{AcS}(\mathrm{V})$ é um sistema que, tal como BUT(V), fortalece levemente $\mathrm{BT}(\mathrm{V})$ (e. g., deriva a reflexividade da identidade mas é conservativo sobre P.A.); de modo que $\mathrm{AcS}(\mathrm{V})$ e BUT(V) estão aparentados em um sentido muito próximo daquele em que $\operatorname{AcM}(\mathrm{V})$ e CUT(V) também estão [cf. p. 112]. O que sugere fortemente uma comparação mais detalhada e profunda entre BUT(V), AcM(VF)+EsqS e AcS(V), todos eles sistemas de citação/descitação (supersistemas de BT(V)) relativamente fracos. 


\section{VI}

\section{Sistemas baseados em definições parciais alternativas}

Uma das características mais interessantes e bem-vindas das DVPs é que podemos modificá-las e, por meio dessas versões alternativas, introduzir, tomando o capítulo V como paradigma, novos sistemas de acumulação da verdade.

Estudaremos nesse capítulo dois exemplos dessas modificações: a) a primeira permitirá estabelecer várias propriedades auto-referenciais do predicado de verdade; b) a segunda permitirá estabelecer enunciados gerais de leis da lógica (tudo isso em sistemas que são conservativos sobre P.A.); forçando, em ambos os casos, os limites de nossos sistemas de acumulação (e, incidentalmente, das abordagens deflacionistas do predicado de verdade).

Não obstante, os sistemas desse capítulo devem ser tomados tentativamente como concretização da ideia de acumulação. Por exemplo, o sistema $\operatorname{AcRM(V)~é~}$ resultado de uma série de correções e aprimoramentos de sistemas anteriores (condenados ao limbo dos rejeitados); contudo, seria temerário, uma vez que existe uma infinidade de questões não respondidas, afirmar que, em $\operatorname{AcRM}(V)$, encontramos a formulação mais adequada da ideia de acumulação auto-referencial. Nossa esperança é que as cláusulas de acumulação introduzidas nesse capítulo sejam, quando não totalmente adequadas, ao menos suficientemente maleáveis para permitir um futuro refinamento das ideias subjacentes aos sistemas aqui expostos.

\section{$\S 1.0$ sistema formal $\mathrm{AcRM}(\mathrm{V})$}

Modificando nossas DPVs, podemos definir sistemas claramente auto-referenciais, tanto no sentido preciso de $\mathrm{V}(\lceil\mathrm{V}(\lceil\tau=\tau\rceil)\rceil)[\mathrm{cf}$. V-1.18, p. 109] quanto no sentido mais frouxo, por exemplo, de $\forall x\left(\operatorname{SENT}_{n}(\mathrm{~V})(x) \wedge \mathrm{V}(x) \rightarrow \mathrm{V}(\dot{\mathrm{V}}(x))\right)^{(1)}$. Essa será nossa tarefa nessa seção. 
Introduziremos a base de nossas formas alternativas das DPVs:

\subsection{Definição de $\mathrm{V}_{0}^{\mathrm{R}}(x)$.}

a) $\mathrm{V}_{0}^{\mathrm{R}}(x)$ será uma abreviação de $\mathrm{V}_{0}(x) \vee\left(\dot{\mathrm{V}}(x) \wedge \mathrm{V}_{0}(\mathrm{PV}(x))\right)$.

Devemos notar, então, que (se P.A. é consistente) P.A. $\forall \mathrm{V}_{0}(\lceil\mathrm{~V}(\lceil\tau=\tau\rceil)\rceil)$ e que P.A. $\forall \mathrm{V}_{1}(\lceil\mathrm{~V}(\lceil\tau=\tau\rceil)\rceil)$; entretanto, P.A. $\vdash \mathrm{V}_{0}^{\mathrm{R}}(\lceil\mathrm{V}(\lceil\tau=\tau\rceil)\rceil)$, uma vez que $\dot{\mathrm{V}}(\lceil\mathrm{V}(\lceil\tau=\tau\rceil)\rceil)$, $\operatorname{PV}(\lceil\mathrm{V}(\lceil\tau=\tau\rceil)\rceil) \approx\lceil\tau=\tau\rceil$ e $\mathrm{V}_{0}(\lceil\tau=\tau\rceil)$ são todos teoremas de P.A.-ou seja, uma vez que as contrapartidas formais de “' $\mathrm{V}(\lceil\tau=\tau\rceil)$ ' é uma fórmula atômica do tipo ' $\mathrm{V}(\cdots)$ '”, “' $\lceil\tau=\tau\rceil$ ' é o subtermo principal de ' $\mathrm{V}(\lceil\tau=\tau\rceil)$ '” e “' $\tau=\tau$ ' é uma verdade atômica em N" são deriváveis em P.A..

A introdução da forma geral das definições parciais modificadas dessa seção demandará alterações mais profundas nas subfórmulas das DPVs.

Deveríamos, por exemplo, ser capazes de derivar $\mathrm{V}_{1}^{\mathrm{R}}(\lceil\sim \mathrm{V}(\lceil\overline{2}=\overline{3}\rceil)\rceil)$ ou, talvez, $\mathrm{V}_{2}^{\mathrm{R}}(\lceil\sim \mathrm{V}(\lceil\overline{2}=\overline{3}\rceil)\rceil)$ em P.A.; o que não aconteceria se tomássemos ingênua e imediatamente ' $\mathrm{V}_{n}^{\mathrm{R}}(x) \vee\left(\dot{\mathrm{V}}(x) \wedge \mathrm{V}_{n}^{\mathrm{R}}(\mathrm{PV}(x))\right)$ ' como $\mathrm{V}_{n+1}^{\mathrm{R}}(x)$. Devemos, portanto, levar em conta as "ocorrências oblíquas" dos símbolos lógicos e de 'V'-tematizamos rapidamente esse tipo de obliquidade em V-1.19 [cf. p. 109].

\subsection{Definição das versões oblíquas de $\mathrm{SENT}_{n}(\mathrm{~V})(x)$.}

a) Primeiro, escreveremos $\backslash \operatorname{SENT}(\mathrm{V})_{0} \backslash(x)$ no lugar de $\operatorname{SENT} \doteq(x) \vee \operatorname{SENT} \dot{\leqslant}(x) \vee$ $(\operatorname{SENT} \dot{V}(x) \wedge(\operatorname{SENT} \doteq(\mathrm{PV}(x)) \vee \operatorname{SENT} \dot{=}(\mathrm{PV}(x))))[$ cp. III-6.4 p. 67];

1. Esse é um bom momento para relembrarmos alguns aspectos de nossas convenções notacionais. Vamos comparar as fórmulas $\forall x\left(\operatorname{SENT}_{n}(\mathrm{~V})(x) \wedge \mathrm{V}(x) \rightarrow \mathrm{V}(\dot{\mathrm{V}}(x))\right)$ e $\forall x(\operatorname{SENT}(\mathrm{V})(x) \rightarrow \operatorname{SENT}(x) \vee \dot{\mathrm{V}}(x))$. No primeiro caso, ' $\dot{\mathrm{V}}(x)$ ' se refere à fórmula $\dot{\mathrm{V}}(x) \approx y$ [cf. III-6.3.a), pp. 66-67] que é o gráfico de uma certa função, $\dot{\mathrm{V}}(x) \approx y$ é uma contrapartida formal de " $y$ é a aplicação do predicado ' $\mathrm{V}$ ' a $x$ "; de modo que $\forall x\left(\operatorname{SENT}_{n}(\mathrm{~V})(x) \wedge \mathrm{V}(x) \rightarrow\right.$ $\mathrm{V}(\dot{\mathrm{V}}(x)))$ é, por sua vez, uma contrapartida de "o resultado da aplicação do predicado ' $\mathrm{V}$ ' ao número de Gödel de uma sentença verdadeira de complexidade $n$ é uma sentença verdadeira”, ' $\dot{V}(x)$ ' é, nesse caso específico, entendido como um pseudo-termo [cf. discussão, p. 61]. No segundo caso, ' $\dot{V}(x)$ ' se refere diretamente à fórmula $\dot{\mathrm{V}}(x)$ [cf. III-6.3.c), pp. 66-67] que é uma contrapartida formal de " $x$ é uma fórmula atômica do tipo 'V( $\ldots)^{\text {'”; }}$ de modo que $\forall x(\operatorname{SENT}(\mathrm{V})(x) \rightarrow \operatorname{SENT}(x) \vee \dot{\mathrm{V}}(x))$ é, por sua vez, uma contrapartida de "as sentenças de $\mathrm{L}_{\text {P.A.V. }}$ ou são sentenças de $L_{\text {P.A. }}$ ou são do tipo ' $V(\ldots)$ '” (o que é falso). 
b) E, então, escreveremos $\backslash \operatorname{SENT}(\mathrm{V})_{n+1} \backslash(x)$ no lugar de $\backslash \operatorname{SENT}(\mathrm{V})_{n} \backslash(x) \vee \alpha_{1} \vee$ $\alpha_{2} \vee \alpha_{3} \vee \alpha_{4} \vee \alpha_{5} \vee \alpha_{6}$

c) Onde $\alpha_{1}$ é $\left(\operatorname{SENT}(\mathrm{V}) \dot{\sim}(x) \wedge \backslash \operatorname{SENT}(\mathrm{V})_{n} \backslash(\mathrm{P} \dot{\sim}(x))\right)$;

d) $\alpha_{2}$ é $\left.\left(\operatorname{SENT}(\mathrm{V}) \dot{\vee}(x) \wedge \backslash \operatorname{SENT}(\mathrm{V})_{n} \backslash(\operatorname{PP} \dot{\vee}(x))\right) \wedge \backslash \operatorname{SENT}(\mathrm{V})_{n} \backslash(\operatorname{PS} \dot{\vee}(x))\right)$;

e) $\alpha_{3}$ é $\left.\left(\operatorname{SENT}(\mathrm{V}) \dot{\lambda}(x) \wedge \backslash \operatorname{SENT}(\mathrm{V})_{n} \backslash(\operatorname{PP} \dot{\lambda}(x))\right) \wedge \backslash \operatorname{SENT}(\mathrm{V})_{n} \backslash(\operatorname{PS} \dot{\lambda}(x))\right)$;

f) $\alpha_{4}$ é $\left(\operatorname{SENT}(\mathrm{V}) \dot{\exists}(x) \wedge \backslash \operatorname{SENT}(\mathrm{V})_{n} \backslash(\operatorname{INS}(x,\lceil\overline{0}=\overline{0}\rceil))\right)$;

g) $\alpha_{5}$ é $\left(\operatorname{SENT}(\mathrm{V}) \dot{\forall}(x) \wedge \backslash \operatorname{SENT}(\mathrm{V})_{n} \backslash(\operatorname{INS}(x,\lceil\overline{0}=\overline{0}\rceil))\right)$;

h) $\alpha_{6}$ é $\left(\operatorname{SENTV}(x) \wedge \backslash \operatorname{SENT}(\mathrm{V})_{n} \backslash(\operatorname{PV}(x))\right)$.

Devemos notar que, em ' $\mathrm{SENT}(\mathrm{V})_{n} \backslash(x)$ ', ' $n$ ' está se referindo às etapas de introdução de conectivos, quantificadores e 'V's e não à quantidade desses símbolos, o que é uma vantagem técnica do ponto de vista da notação.

Alguns exemplos serão úteis:

\subsection{Exemplos.}

a) P.A. $\vdash \backslash \operatorname{SENT}(\mathrm{V})_{0} \backslash(\lceil\mathrm{V}(\lceil\overline{2}=\overline{2}\rceil)\rceil)$;

b) contudo, P.A. $\| \backslash \operatorname{SENT}(\mathrm{V})_{0} \backslash(\lceil\mathrm{V}(\lceil\mathrm{V}(\lceil\overline{2}=\overline{2}\rceil)\rceil)\rceil)$ e P.A. $H \backslash \operatorname{SENT}(\mathrm{V})_{0} \backslash(\lceil\mathrm{V}(\overline{10})\rceil)$,

c) embora, P.A. $\vdash \operatorname{SENT}(\mathrm{V})_{0}(\lceil\mathrm{~V}(\lceil\mathrm{~V}(\lceil\overline{2}=\overline{2}\rceil)\rceil)\rceil)$ e P.A. $\vdash \operatorname{SENT}(\mathrm{V})_{0}(\lceil\mathrm{~V}(\overline{10})\rceil)$.

d) P.A. $\vdash \backslash \operatorname{SENT}(\mathrm{V})_{1} \backslash(\lceil\mathrm{V}(\lceil\mathrm{V}(\lceil\overline{2}=\overline{2}\rceil)\rceil)\rceil)$;

e) P.A. $\vdash \operatorname{SENT}(\mathrm{V})_{1} \backslash(\lceil\sim \mathrm{V}(\lceil\overline{2}=\overline{3}\rceil)\rceil)$;

f) e, portanto, P.A. $+\backslash \operatorname{SENT}(\mathrm{V})_{2} \backslash(\lceil\mathrm{V}(\lceil\mathrm{V}(\lceil\overline{2}=\overline{2}\rceil)\rceil) \vee \sim \mathrm{V}(\lceil\overline{2}=\overline{3}\rceil)\rceil)$.

\subsection{Definição das versões oblíquas específicas de $\operatorname{SENT}_{n}(\mathrm{~V})(x)$.}

a) Escreveremos $\backslash \operatorname{SENT}(\mathrm{V}) \dot{\sim}_{n} \backslash(x)$ no lugar de $\operatorname{SENT}(\mathrm{V}) \dot{\sim}(x) \wedge \backslash \operatorname{SENT}(\mathrm{V})_{n} \backslash(x)$. O mesmo procedimento deve ser aplicado às demais constantes lógica.

Por exemplo:

b) $\backslash \operatorname{SENT}(\mathrm{V}) \dot{\vee}_{n} \backslash(x)$ no lugar de $\operatorname{SENT}(\mathrm{V}) \dot{\vee}(x) \wedge \backslash \operatorname{SENT}(\mathrm{V})_{n} \backslash(x)$;

c) $\backslash \operatorname{SENT}(\mathrm{V}) \dot{\exists}_{n} \backslash(x)$ no lugar de $\operatorname{SENT}(\mathrm{V}) \dot{\exists}(x) \wedge \backslash \operatorname{SENT}(\mathrm{V})_{n} \backslash(x)$. 
Podemos, então, introduzir a forma geral de nossas definições parcias enraizadas de verdade ou RVs:

\subsection{Definição da forma geral das RVs.}

a) Escreveremos $\mathrm{V}_{n+1}^{\mathrm{R}}(x)$ no lugar de $\mathrm{V}_{n}^{\mathrm{R}}(x) \vee \alpha_{1} \vee \alpha_{2} \vee \alpha_{3} \vee \alpha_{4} \vee \alpha_{5} \vee \alpha_{6}$;

b) Onde $\alpha_{1}$ é $\left(\backslash \operatorname{SENT}(\mathrm{V}) \dot{\sim}_{n+1} \backslash(x) \wedge \sim \mathrm{V}_{n}^{\mathrm{R}}(\mathrm{P} \sim(x))\right)$;

c) $\alpha_{2}$ é $\left(\backslash \operatorname{SENT}(\mathrm{V}) \dot{\vee}_{n+1} \backslash(x) \wedge\left(\mathrm{V}_{n}^{\mathrm{R}}(\mathrm{PP} \dot{\vee}(x)) \vee \mathrm{V}_{n}^{\mathrm{R}}(\mathrm{PS} \dot{\vee}(x))\right)\right)$;

d) $\alpha_{3}$ é $\left(\backslash \operatorname{SENT}(\mathrm{V}) \dot{\wedge}_{n+1} \backslash(x) \wedge\left(\mathrm{V}_{n}^{\mathrm{R}}(\mathrm{PP} \dot{\wedge}(x)) \wedge \mathrm{V}_{n}^{\mathrm{R}}(\mathrm{PS} \dot{\wedge}(x))\right)\right)$;

e) $\alpha_{4}$ é $\left(\backslash \operatorname{SENT}(\mathrm{V}) \dot{\exists}_{n+1} \backslash(x) \wedge \exists y \mathrm{~V}_{n}^{\mathrm{R}}(\operatorname{INS}(x, y))\right)$;

f) $\alpha_{5}$ é $\left(\backslash \operatorname{SENT}(\mathrm{V}) \dot{\forall}_{n+1} \backslash(x) \wedge \forall y \mathrm{~V}_{n}^{\mathrm{R}}(\operatorname{INS}(x, y))\right)$;

g) $\alpha_{6}$ é $\left(\backslash \operatorname{SENT}(\mathrm{V}) \dot{\mathrm{V}}_{n+1} \backslash(x) \wedge \mathrm{V}_{n}^{\mathrm{R}}(\mathrm{PV}(x))\right)$.

A grande novidade da construção das RVs em relação às DPVs [cp. IV-5.3, pp. 94-95] são, portanto, os novos predicados "oblíquos" $\backslash \cdots \backslash(x)$ e nossa última cláusula $\alpha_{6}$ que permitirão um tratamento auto-referencial adequado do predicado de verdade.

1.6. Definição. As fórmulas $\mathrm{V}_{0}^{\mathrm{R}}(x), \mathrm{V}_{1}^{\mathrm{R}}(x), \ldots, \mathrm{V}_{n}^{\mathrm{R}}(x), \ldots$ serão chamadas definições parciais enraizadas de verdade ou, mais sucintamente, RVs.

$\mathrm{O}$ 'R' em 'RVs' remete ao radical 'raiz' em 'enraizadas' para opô-las às EVs ou definições parciais estruturais de verdade da próxima seção. Nosso ‘enraizadas' remete, por sua vez, à noção kripkeana de "grounded sentence"(2) [Kripke, 1975, pp. 693-694] e não é totalmente alheia à noção conjunto-teorética de "well-founded".

A hierarquia induzida pela definição acima é à primeira vista um pouco estranha; pois, devemos "contar" as ocorrências oblíquas dos símbolos lógicos e dos predicados de verdade; entretanto, essa "estranheza", como deve estar claro agora, é característica das RVs.

2. O que não quer dizer absolutamente que o sistema $\operatorname{AcMR}(\mathrm{V})$ dessa seção tenha, em princípio, relação com alguma das teorias propostas por ou inspiradas em "Outline of a Theory of Truth" de Kripke [para nossas "inspirações" diretas cf. p. 11], embora um estudo nessa direção seja, obviamente, bem-vindo. 
Temos, por exemplo, que

a) P.A. $+\mathrm{V}_{0}^{\mathrm{R}}(\lceil\mathrm{V}(\lceil\overline{2}=\overline{2}\rceil)\rceil)$ [cf. 1.1, p. 125]

b) e, desde que P.A. $\vdash \sim \mathrm{V}_{0}^{\mathrm{R}}(\lceil\mathrm{V}(\lceil\overline{2}=\overline{3}\rceil)\rceil) \wedge \backslash \operatorname{SENT}(\mathrm{V}) \dot{\sim}_{1} \backslash(\lceil\sim \mathrm{V}(\lceil\overline{2}=\overline{3}\rceil)\rceil)$,

c) que P.A. $\vdash V_{1}^{R}(\lceil\sim V(\lceil\overline{2}=\overline{3}\rceil)\rceil)$ [cf. 1.5.b), p. 127].

d) Portanto, temos que P.A. $\vdash \mathrm{V}_{2}^{\mathrm{R}}(\lceil\mathrm{V}(\lceil\overline{2}=\overline{2}\rceil) \wedge \sim \mathrm{V}(\lceil\overline{2}=\overline{3}\rceil)\rceil)$ [cf. 1.5.d), p. 127]

e) e, também, que P.A. $\vdash \mathrm{V}_{2}^{\mathrm{R}}(\lceil\mathrm{V}(\lceil\sim \mathrm{V}(\lceil\overline{2}=\overline{3}\rceil)\rceil)\rceil)$ [cf. 1.5.g), p. 127].

Devemos notar que, em ' $\mathrm{V}(\lceil\overline{2}=\overline{2}\rceil) \wedge \sim \mathrm{V}(\lceil\overline{2}=\overline{3}\rceil)$ ', temos uma ocorrência de ' $\wedge$ ' e de ' $\sim$ ', e duas ocorrências de 'V'; mas que, em ' $\mathrm{V}(\lceil\sim \mathrm{V}(\lceil\overline{2}=\overline{3}\rceil)\rceil)$ ', há apenas uma ocorrência de ' $\mathrm{V}$ ', uma vez que ' $\lceil\sim \mathrm{V}(\lceil\overline{2}=\overline{3}\rceil)\rceil$ ' é um numeral. De modo que ' $\mathrm{V}(\lceil\overline{2}=\overline{3}\rceil)$ ' e 'V( $(\lceil\sim \mathrm{V}(\lceil\overline{2}=\overline{3}\rceil)\rceil)$ ', embora tenham uma mesma complexidade lógica, possuem complexi-dades diferentes do ponto vista da hierárquia induzida pelas RVs.

Tal como no caso das DPVs, temos que:

1.7. Teorema. Para qualquer número natural $n$ tal que $\alpha$ é uma sentença de $L_{\text {P.A. }}$. de complexidade oblíqua $n$, P.A. $\vdash \alpha \leftrightarrow \mathrm{V}_{n}^{\mathrm{R}}([\alpha])$.

O teorema análogo a 1.7 para todas as sentenças de $L_{\text {P.A.V. }}$, e que terá sentido no sistema que definiremos em 1.9, é, além de falso, mais complicado; de modo que deixaremos essa questão em aberto. Entretanto, como já deve estar claro pelo exemplo logo acima, as RVs nos permitiram ir muito além das sentenças de $L_{\text {P.A. }}$.

1.8. Definição. As sentenças $\forall x\left(\mathrm{~V}_{n}^{\mathrm{R}}(x) \rightarrow \mathrm{V}(x)\right)$, onde $n$ é um número natural, serão chamadas cláusulas de acumulação enraizadas-positivas ou, mais sucintamente, CARPs.

1.9. Definição. $A c R M(V)$ é o sistema cujos axiomas são os axiomas de P.A. para $\mathrm{L}_{\text {P.A. }}$ e as sentenças $\forall x\left(\mathrm{~V}_{n}^{\mathrm{R}}(x) \rightarrow \mathrm{V}(x)\right)$, para todo $n$. 
$\operatorname{AcRM}(\mathrm{V})$ é chamado sistema minimal (restrito) da acumulação enraizadapositiva.

Dada qualquer derivação $\alpha=\left\langle\alpha_{1}, \alpha_{2}, \ldots, \alpha_{j}\right\rangle$ em AcRM(V) cuja RV de maior complexidade é $\mathrm{V}_{i}^{\mathrm{R}}$, podemos, então, definir uma substituição $[\beta] \mathrm{V} / \mathrm{V}_{i}^{\mathrm{R}}$ das ocorrências de $\mathrm{V}$ por $\mathrm{V}_{i}^{\mathrm{R}}$ em $\beta$ (analogamente ao que fizemos em $\mathbf{V}$-§1 [pp. 102-103]).

Nesse caso, temos que $[\mathrm{V}(\lceil\mathrm{V}(\lceil\overline{2}=\overline{2}\rceil)\rceil)] \mathrm{V} / \mathrm{V}_{2}^{\mathrm{R}}$ é $\mathrm{V}_{2}^{\mathrm{R}}(\lceil\mathrm{V}(\lceil\overline{2}=\overline{2}\rceil)\rceil)$-e não, o que é muito importante, $\mathrm{V}_{2}^{\mathrm{R}}\left(\left\lceil\mathrm{V}_{2}^{\mathrm{R}}(\lceil\overline{2}=\overline{2}\rceil)\right\rceil\right)$-, de modo que as "ocorrências oblíquas" de $\mathrm{V}$ não são relevantes para $[\beta] \mathrm{V} / \mathrm{V}_{i}^{\mathrm{R}}$ (a correção do nosso próximo argumento depende disso).

Definiremos [cp. $/ \alpha /(1)$ p. 102], primeiramente, $/ \alpha /^{\mathrm{R}}(1)$ :

a) $/ \alpha /^{\mathrm{R}}(1)=\left\langle\left[\alpha_{1}\right]^{\mathrm{V}} / \mathrm{V}_{i}^{\mathrm{R}}\right\rangle$ se $\alpha_{1}$ é um axioma de P.A.;

b) $/ \alpha /^{\mathrm{R}}(1)=\beta$, onde $\beta=\left\langle\beta_{1}, \beta_{2}, \ldots,\left[\alpha_{1}\right]^{\mathrm{V}} / \mathrm{V}_{i}^{\mathrm{R}}\right\rangle$ é uma derivação de $\forall x\left(\mathrm{~V}_{k}^{\mathrm{R}}(x) \rightarrow\right.$ $\left.\mathrm{V}_{i}^{\mathrm{R}}(x)\right)$ em P.A., se $\alpha_{1}$ é $\forall x\left(\mathrm{~V}_{k}^{\mathrm{R}}(x) \rightarrow \mathrm{V}(x)\right)$, ou seja, se $\alpha_{1}$ é uma CARP.

Definiremos [cp. $/ \alpha /(n+1)$ p. 102], agora, $/ \alpha /^{\mathrm{R}}(n+1)$ :

a) $/ \alpha /^{\mathrm{R}}(n+1)=$ a concatenação entre $/ \alpha /^{\mathrm{R}}(n)$ e $\left\langle\left[\alpha_{n+1}\right]^{\mathrm{V}} / \mathrm{V}_{i}^{\mathrm{R}}\right\rangle$ se $\alpha_{n+1}$ é um axioma de P.A. ou se $\alpha_{n+1}$ é resultado da aplicação do modus ponens ou se $\alpha_{n+1}$ é resultado da aplicação da generalização universal.

b) $/ \alpha /^{\mathrm{R}}(n+1)=$ a concatenação entre $/ \alpha /^{\mathrm{R}}(n)$ e $\beta$, onde $\beta=\left\langle\beta_{1}, \beta_{2}, \ldots,\left[\alpha_{3}\right]^{\mathrm{V}} / \mathrm{V}_{i}^{\mathrm{R}}\right\rangle$ é uma derivação de $\forall x\left(\mathrm{~V}_{k}^{\mathrm{R}}(x) \rightarrow \mathrm{V}_{i}^{\mathrm{R}}(x)\right)$ em P.A., se $\alpha_{n+1}$ é $\forall x\left(\mathrm{~V}_{k}^{\mathrm{R}}(x) \rightarrow \mathrm{V}(x)\right)$.

1.10. Definição. Se $\alpha$ é uma derivação tal como a descrevemos acima; escreveremos $/ \alpha /^{\mathrm{R}}$ no lugar de $/ \alpha /{ }^{\mathrm{R}}(j)$.

Razões análogas às apresentadas em V-\$1 [pp. 102-103] estabelecem:

1.11. Lema. Se $\alpha$ é uma derivação de $\alpha_{j}$ em $\operatorname{AcRM}(V)$ tal que $V_{i}^{R}(x)$ é a $R V$ de maior complexidade de $\alpha$, então $/ \alpha /^{\mathrm{R}}$ será uma derivação de $\left[\alpha_{j}\right]^{\mathrm{V}} / \mathrm{V}_{i}^{\mathrm{R}}$ em P.A.. 
E, portanto, que:

1.12. Teorema. AcRM(V) é conservativo sobre P.A..

Exemplificaremos nosso procedimento anterior:

a) $\operatorname{AcRM}(V)+\overline{2}=\overline{2}$;

b) $\operatorname{AcRM}(V)+V_{2}^{R}(\lceil\overline{2}=\overline{2}\rceil)$, de a);

c) $\operatorname{AcRM}(\mathrm{V})+\dot{\mathrm{V}}(\lceil\mathrm{V}(\lceil\overline{2}=\overline{2}\rceil)\rceil) \wedge \mathrm{P} \dot{\mathrm{V}}(\lceil\mathrm{V}(\lceil\overline{2}=\overline{2}\rceil)\rceil) \simeq\lceil\overline{2}=\overline{2}\rceil$;

d) $\operatorname{AcRM}(\mathrm{V}) \vdash \mathrm{V}_{0}^{\mathrm{R}}(\lceil\mathrm{V}(\lceil\overline{2}=\overline{2}\rceil)\rceil)$, de a) e c);

e) $\operatorname{AcRM}(\mathrm{V})+\mathrm{V}(\lceil\mathrm{V}(\lceil\overline{2}=\overline{2}\rceil)\rceil)$, de d) e $\forall x\left(\mathrm{~V}_{0}^{\mathrm{R}}(x) \rightarrow \mathrm{V}(x)\right)$;

f) $\operatorname{AcRM}(\mathrm{V})+\mathrm{V}(\lceil\mathrm{V}(\lceil\overline{2}=\overline{2}\rceil)\rceil) \wedge \mathrm{V}_{2}^{\mathrm{R}}(\lceil\overline{2}=\overline{2}\rceil)$, de b) e e $)$.

O argumento a)-f) pode ser convertido em uma derivação rigorosa em $\operatorname{AcRM}(\mathrm{V})$; o mesmo pode ser dito da "transformação" /a)-f) $/{ }^{R}$ em relação a P.A.:

a) P.A. $\vdash \overline{2}=\overline{2}$;

b) P.A. $\vdash \mathrm{V}_{2}^{\mathrm{R}}(\lceil\overline{2}=\overline{2}\rceil)$, de a);

c) P.A. $\vdash \dot{\mathrm{V}}(\lceil\mathrm{V}(\lceil\overline{2}=\overline{2}\rceil)\rceil) \wedge \mathrm{PV}(\lceil\mathrm{V}(\lceil\overline{2}=\overline{2}\rceil)\rceil) \simeq\lceil\overline{2}=\overline{2}\rceil$;

d) P.A. $\vdash \mathrm{V}_{0}^{\mathrm{R}}(\lceil\mathrm{V}(\lceil\overline{2}=\overline{2}\rceil)\rceil)$, de a) e c);

e) P.A. $\vdash \mathrm{V}_{2}^{\mathrm{R}}(\lceil\mathrm{V}(\lceil\overline{2}=\overline{2}\rceil)\rceil)$, de d) e $\forall x\left(\mathrm{~V}_{0}^{\mathrm{R}}(x) \rightarrow \mathrm{V}_{2}^{\mathrm{R}}(x)\right)$;

f) P.A. $\vdash V_{2}^{R}(\lceil V(\lceil\overline{2}=\overline{2}\rceil)\rceil) \wedge V_{2}^{R}([\overline{2}=\overline{2}\rceil)$, de b) e e $)$.

Vale notar que $\mathrm{V}_{1}^{\mathrm{R}}(\lceil\mathrm{V}(\lceil\overline{2}=\overline{2}\rceil)\rceil)$, é derivável em P.A., mas, devido à complexidade de $\mathrm{V}_{0}^{\mathrm{R}}(\lceil\overline{2}=\overline{2}\rceil)$, P.A. $\forall \mathrm{V}_{1}^{\mathrm{R}}\left(\left\lceil\mathrm{V}_{0}^{\mathrm{R}}(\lceil\overline{2}=\overline{2}\rceil)\right\rceil\right)$; ainda assim, presumivelmente, temos que P.A. $\vdash \mathrm{V}_{100}^{\mathrm{R}}\left(\left\lceil\mathrm{V}_{0}^{\mathrm{R}}(\lceil\overline{2}=\overline{2}\rceil)\right\rceil\right)$.

Um resultado bastante simples, embora característico, de $\operatorname{AcMR}(V)$ é:

1.13. Teorema. Para qualquer sentença $\alpha$ e número natural $n$, se $\alpha$ é um teorema de P.A., então AcRM(V) $\vdash \mathrm{V}(\lceil\cdots \mathrm{V}(\lceil\mathrm{V}(\lceil\alpha\rceil)\rceil) \cdots\rceil)$. 
Segue-se, então, de 1.13 que:

1.14. Corolário. AcRM(V) é auto-referencial [cp. V-1.19, p. 109].

Outros resutados em $\mathrm{AcRM}(\mathrm{V})$ :

\subsection{Teoremas.}

a) $\operatorname{AcRM}(\mathrm{V})+\mathrm{V}(\lceil\exists x \mathrm{~V}(x)\rceil)$;

b) $\operatorname{AcRM}(\mathrm{V})+\mathrm{V}(\lceil\exists x \sim \mathrm{V}(x)\rceil)$;

c) $\operatorname{AcRM}(\mathrm{V})+\mathrm{V}(\lceil\forall x(\mathrm{~V}(x \doteq x))])$, onde $\forall x(\mathrm{~V}(x \doteq x))$ é descrita mais precisamente como $\forall x \forall y(\dot{\doteq}(x, x) \approx y \rightarrow \mathrm{V}(y))$.

\section{Provas.}

a)

1) Uma vez que P.A. $\vdash \mathrm{V}_{0}^{\mathrm{R}}(\lceil\mathrm{V}(\lceil\overline{0}=\overline{0}\rceil)\rceil) \wedge \mathrm{INS}(\lceil\exists x \mathrm{~V}(x)\rceil,\lceil\overline{0}=\overline{0}\rceil) \simeq\lceil\mathrm{V}(\lceil\overline{0}=\overline{0}\rceil)\rceil$;

2) P.A. $\vdash \mathrm{V}_{1}^{\mathrm{R}}(\lceil\exists x \mathrm{~V}(x)\rceil)$, pela cláusula 1.5.e) [p. 127].

3) $\mathrm{E}$, portanto, de $\operatorname{AcRM}(\mathrm{V}) \vdash \forall x\left(\mathrm{~V}_{1}^{\mathrm{R}}(x) \rightarrow \mathrm{V}(x)\right)$;

4) segue-se, então, $\operatorname{AcRM}(\mathrm{V})+\mathrm{V}(\lceil\exists x \mathrm{~V}(x)\rceil)$.

b)

1) P.A. $\vdash \mathrm{V}_{1}^{\mathrm{R}}(\lceil\sim \mathrm{V}(\lceil\overline{2}=\overline{3}\rceil)\rceil)$;

2) P.A. $\vdash \mathrm{V}_{2}^{\mathrm{R}}(\lceil\exists x \sim \mathrm{V}(x)\rceil)$, pela cláusula 1.5.e).

3) $\mathrm{E}$, portanto, $\mathrm{AcRM}(\mathrm{V}) \vdash \mathrm{V}(\lceil\exists x \sim \mathrm{V}(x)\rceil)$.

c)

1) P.A. $\vdash \forall y \forall z\left(\operatorname{INS}(\lceil\forall x(\mathrm{~V}(x \dot{\doteq} x))], y) \simeq z \rightarrow \mathrm{V}_{0}^{\mathrm{R}}(z)\right)$;

2) P.A. $\vdash \mathrm{V}_{1}^{\mathrm{R}}(\lceil\forall x(\mathrm{~V}(x \doteq x))])$, pela cláusula 1.5.f).

3) $\mathrm{E}$, portanto, $\operatorname{AcRM}(\mathrm{V})+\mathrm{V}(\lceil\forall x(\mathrm{~V}(x \doteq x))])$. 


\section{A. Extensões do modelo padrão para $\mathrm{AcRM}(\mathrm{V})$}

Preliminarmente, introduziremos alguns conjuntos e operações auxiliares:

\subsection{Definições auxiliares (fechos de enraizamento).}

a) $\left.\backslash \mathrm{L}\right|_{n}={ }_{\text {df. }}|| \operatorname{SENT}(\mathrm{V})_{n} \backslash(x) \mid=_{\text {df. }}$ o conjunto dos números de Gödel de sentenças de complexidade oblíqua $\leqslant n$;

b) $[\mathrm{A}]_{n}^{\sim}={ }_{\text {df. }}\left\{\sim \alpha|g(\alpha) \in \backslash \mathrm{L}|_{n}\right.$ e $\left.\alpha \notin \mathrm{A}\right\}$;

c) $[\mathrm{A}]_{n}^{\vee}=$ df. $_{\text {. }}\left\{\alpha \vee \beta|g(\alpha) \in \backslash \mathrm{L}|_{n}, g(\beta) \in \backslash \mathrm{L}_{n}\right.$ e ou $\alpha \in \mathrm{A}$ ou $\left.\beta \in \mathrm{A}\right\}$;

d) $[\mathrm{A}]_{n}^{\wedge}={ }_{\text {df. }}\left\{\alpha \wedge \beta|g(\alpha) \in \backslash \mathrm{L}|_{n},\left.g(\beta) \in \backslash \mathrm{L}\right|_{n}, \alpha \in \mathrm{A}\right.$ e $\left.\beta \in \mathrm{A}\right\}$;

e) $[\mathrm{A}]_{n}^{\exists}={ }_{\text {df. }}\left\{\exists v \alpha \mid\right.$ existe $\tau$ tal que $\left.g\left(\alpha^{V} / \tau\right) \in \backslash \mathrm{L}\right|_{n}$ e $\left.\alpha^{\mathrm{V}} / \tau \in \mathrm{A}\right\}$;

f) $[\mathrm{A}]_{n}^{\forall}=$ df. $_{\text {. }}\left\{\forall v \alpha \mid\right.$ para todo termo $\tau,\left.g\left(\alpha^{V} / \tau\right) \in \backslash \mathrm{L}\right|_{n}$ e $\left.\alpha^{\mathrm{V}} / \tau \in \mathrm{A}\right\}$;

g) $[\mathrm{A}]_{n}==_{\mathrm{df} .}[\mathrm{A}]_{n}^{\sim} \cup[\mathrm{A}]_{n}^{\vee} \cup[\mathrm{A}]_{n}^{\wedge} \cup[\mathrm{A}]_{n}^{\exists} \cup[\mathrm{A}]_{n}^{\forall}$.

A ideia de enraizamento presente nas RVs pode, então, ser aplicada ao conjunto das verdades do modelo padrão da aritmética:

\subsection{Definição do conjunto padrão de $\operatorname{AcRM}(\mathrm{V})$.}

a) Seja $\hat{V}_{0}={ }_{\text {df. }} \hat{V} \cup\{\mathrm{V}(\lceil\alpha\rceil) \mid \alpha \in \hat{V}\}$;

b) $\hat{\mathrm{V}}_{1}=\mathrm{dff}_{\mathrm{df}} \hat{\mathrm{V}}_{0} \cup\left[\hat{\mathrm{V}}_{0}\right]_{0} \cup\left\{\mathrm{V}(\lceil\alpha\rceil) \mid \alpha \in \hat{\mathrm{V}}_{0}\right\}$;

c) $\hat{\mathrm{V}}_{2}={ }_{\mathrm{df}} \hat{\mathrm{V}}_{1} \cup\left[\hat{\mathrm{V}}_{1}\right]_{1} \cup\left\{\mathrm{V}(\lceil\alpha\rceil) \mid \alpha \in \hat{\mathrm{V}}_{1}\right\}$.

Em termos gerais:

d) $\hat{\mathrm{V}}_{n+1}==_{\text {df. }} \hat{\mathrm{V}}_{n} \cup\left[\hat{\mathrm{V}}_{n}\right]_{n} \cup\left\{\mathrm{V}(\lceil\alpha\rceil) \mid \alpha \in \hat{\mathrm{V}}_{n}\right\}$;

e) e, finalmente, $\hat{\mathrm{V}}_{\omega}=_{\text {df. }} \cup\left\{\hat{\mathrm{V}}_{n} \mid n \in \omega\right\}, \hat{\mathrm{V}}_{\omega}$ é o enraizamento de $\hat{\mathrm{V}}$.

Podemos, agora, estabelecer que:

1.18. Definição. O modelo padrão $\mathbb{N}\left(\hat{V}_{\omega}\right)$ de $\operatorname{AcRM}(V)$ interpretará as constantes aritméticas do mesmo modo que $\mathbb{N}$ e $\mathrm{V}$ como $\left|\hat{\mathrm{V}}_{\omega}\right|$, ou seja, como $\left\{g(\alpha) \mid \alpha \in \hat{\mathrm{V}}_{\omega}\right\}$.

1.19. Teorema. $\mathbb{N}\left(\hat{V}_{\omega}\right)$ é realmente um modelo de $\operatorname{AcRM}(V)$. 
Alguns exemplos:

a) Uma vez que

1) $\overline{2}=\overline{3} \notin \hat{V}_{0}$;

2) $\mathbb{N}\left(\hat{\mathrm{V}}_{\omega}\right) \neq \overline{2}=\overline{3}$;

3) $\mathrm{V}(\lceil\overline{2}=\overline{3}\rceil) \notin \hat{\mathrm{V}}_{0}$ e, assim, $\sim \mathrm{V}(\lceil\overline{2}=\overline{3}\rceil) \in \hat{\mathrm{V}}_{1}$.

4) Portanto, $\mathbb{N}\left(\hat{V}_{\omega}\right) \notin \mathrm{V}(\lceil\overline{2}=\overline{3}\rceil)$ e $\mathbb{N}\left(\hat{V}_{\omega}\right) \vDash \sim \mathrm{V}(\lceil\overline{2}=\overline{3}\rceil)$.

b) Uma vez que

1) $\forall x(x+\overline{0}=x) \in \hat{\mathrm{V}}_{1}$ e $g(\mathrm{~V}(\lceil\mathrm{~V}(\lceil\overline{2}=\overline{3}\rceil)\rceil)) \in \backslash \mathrm{L}_{1}$;

2) $(\mathrm{V}(\lceil\mathrm{V}(\lceil\overline{2}=\overline{3}\rceil)\rceil)) \vee \forall x(x+\overline{0}=x)) \in \hat{\mathrm{V}}_{2}$;

3) $\mathrm{E}$, portanto, $\mathbb{N}\left(\hat{\mathrm{V}}_{\omega}\right) \vDash \mathrm{V}(\lceil\mathrm{V}(\lceil\overline{2}=\overline{3}\rceil)\rceil) \vee \forall x(x+\overline{0}=x)$.

Notemos que existe uma pequena discrepância entre $\hat{V}_{0}$ e $V_{0}^{\mathrm{R}}(x)$ e outros casos similares: temos, e. g., que $\forall x(x+\overline{0}=x) \in \hat{\mathrm{V}}_{0}$, uma vez que $\forall x(x+\overline{0}=x) \in \hat{\mathrm{V}}$, enquanto que P.A. $H^{\mathrm{R}} \mathrm{V}_{0}^{\mathrm{R}}(\lceil\forall x(x+\overline{0}=x)\rceil)$. Tal discrepância poderia ser sanada; contudo, isso não seria particularmente útil aqui.

1.20. Definição. Na interpretação $\mathbb{N}\left(\hat{V}_{n}\right)$ de $L_{\text {P.A.V. }}$, as constantes aritméticas serão interpretadas como em $\mathbb{N}$ e V como $\left|\hat{\mathrm{V}}_{n}\right|$, ou seja, como $\left\{g(\alpha) \mid \alpha \in \hat{\mathrm{V}}_{n}\right\}$.

1.21. Teorema. Não existe $n$ tal que $\mathbb{N}\left(\hat{V}_{n}\right)$ seja modelo de $\operatorname{AcRM}(\mathrm{V})$.

Uma vez que P.A. é $\Sigma_{0}$-completo (de fato, $\Sigma_{1}$-completo), devemos notar que, embora T.P.A. $(=\{\alpha \mid$ P.A. $\vdash \alpha\}), \hat{V}_{\text {At }}(=\{\alpha \mid \alpha$ é atômica e $\alpha \in \hat{V}\})$ e $\hat{V}$ sejam conjuntos diferentes de sentenças, eles conduzem ao mesmo enraizamento $\hat{V}_{\omega}=$ T.P.A. $\omega=\left(\hat{V}_{\text {At }}\right)_{\omega}$.

É, portanto, trivial que:

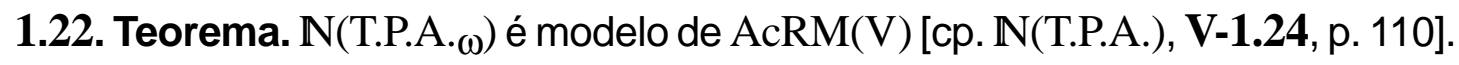


De modo que um possível paralelismo entre as interpretações $\mathbb{N}$ (T.P.A.) [cf. V1.23, p. 110] e $\mathbb{N}($ T.P.A. $\omega)$, análogo àquele entre $\mathbb{N}(\hat{V})$ e $\mathbb{N}\left(\hat{V}_{n}\right)$, não se verifica. De fato, isso se dá por causa da "fase de enraizamento" $\left\{\forall v \alpha \mid\right.$ para todo termo $\tau,\left.g(\alpha \mathrm{V} / \tau) \in \backslash L\right|_{n}$ e $\alpha^{V} / \tau \in$ T.P.A. $_{n}$ \} [cf. 1.16.f), p. 132] pressuposta na construção de T.P.A. ${ }_{n+1}$ e que, por assim dizer, incorpora uma regra $\omega$ no procedimento de enraizamento.

1.23. Teorema. Se $\{\alpha \mid$ P.A. $\vdash \alpha\} \subseteq A$, então $\mathbb{N}\left(A_{\omega}\right)$ é modelo de $\operatorname{AcRM}(V)$, onde V é interpretado como $\left|\mathrm{A}_{\omega}\right|$ (as constantes aritméticas permanacem como em $\mathbb{N}$ ) e $\mathrm{A}_{\omega}$ é o enraizamento de A [cp. V-1.27, p. 111].

Segue-se que:

1.24. Corolário. $\mathbb{N}(\omega)$ é um modelo de $\operatorname{AcRM}(V)$ [cp. V-1.21, p. 110].

Assim, AcRM(V) é compatível com o trivialismo [cp. p. 110].

Além disso, temos que (respondendo parcialmente uma questão da p. 128):

1.25. Teorema. $\operatorname{AcRM}(\mathrm{V}) \Vdash \exists x \sim \mathrm{V}(x)$.

$\mathrm{E}$, portanto, AcRM(V) $\Vdash \mathrm{V}(\lceil\exists x \sim \mathrm{V}(x)\rceil) \rightarrow \exists x \sim \mathrm{V}(x)$, por 1.15b) [p. 131].

Na direção oposta ao trivialismo, temos que:

1.26. Teorema. $S e \hat{V}_{A t} \subseteq A \subseteq T . P . A$., então $\mathbb{N}\left(A_{\omega}\right)$ será um modelo não-dialético de $\operatorname{AcRM}(\mathrm{V})$, ou seja, não existirá sentença $\alpha$ de $\mathrm{L}_{\text {P.A.V. }}$ tal que $\mathbb{N}\left(\mathrm{A}_{\omega}\right) \vDash \mathrm{V}(\lceil\alpha \wedge \sim \alpha\rceil)$.

Seja $\alpha_{1}, \alpha_{2}, \ldots, \alpha_{n}, \ldots$ uma enumeração das sentenças de $L_{P . A . ~}$ (e. g., pela magnitude do número de Gödel associado):

\subsection{Definição da inseminação de $\beta$ limitada por P.A..}

a) Seja $\{\beta\}_{0}=$ df. T.P.A. $\cup\{\beta\} \cup\{V(\lceil\alpha\rceil) \mid \alpha \in$ T.P.A. ou $\alpha=\beta\}$; 
b) $\{\beta\}_{1}==_{\text {df. }}\left\{\alpha \alpha_{1}\right\} \cup\{\beta\}_{0} \cup\left\{\alpha \mid\{\beta\}_{0} \vDash \alpha\right\} \cup\left[\{\beta\}_{0}\right]_{0}^{\sim} \cup\left\{\mathrm{V}(\lceil\alpha\rceil) \mid \alpha \in\{\beta\}_{0}\right\}$,

$$
\begin{aligned}
& \text { no caso de }\left\{\alpha_{1}\right\} \cup\{\beta\}_{0} \notin \overline{0}=\overline{1} ; \\
& ==_{\text {df. }}\{\beta\}_{0} \cup\left\{\alpha \mid\{\beta\}_{0} \vDash \alpha\right\} \cup\left[\{\beta\}_{0}\right]_{0}^{\sim} \cup\left\{\mathrm{V}(\lceil\alpha]) \mid \alpha \in\{\beta\}_{0}\right\}, \\
& \text { no caso de }\left\{\alpha_{1}\right\} \cup\{\beta\}_{0} \vDash \overline{0}=\overline{1} .
\end{aligned}
$$

Em termos gerais:

d) $\{\beta\}_{n+1}==_{\text {df. }}\left\{\alpha_{n}\right\} \cup\{\beta\}_{n} \cup\left\{\alpha \mid\{\beta\}_{n} \vDash \alpha\right\} \cup\left[\{\beta\}_{n}\right]_{n} \cup\left\{\mathrm{V}(\lceil\alpha\rceil) \mid \alpha \in\{\beta\}_{n}\right\}$,

$$
\begin{aligned}
& \text { no caso de }\left\{\alpha_{1}\right\} \cup\{\beta\}_{n} \# \overline{0}=\overline{1} \text {; } \\
& =_{\text {df. }}\{\beta\}_{n} \cup\left\{\alpha \mid\{\beta\}_{n} \vDash \alpha\right\} \cup\left[\{\beta\}_{n}\right]_{n} \cup\left\{\mathrm{V}(\lceil\alpha\rceil) \mid \alpha \in\{\beta\}_{n}\right\} \text {, } \\
& \text { no caso de }\left\{\alpha_{1}\right\} \cup\{\beta\}_{n} \vDash \overline{0}=\overline{1} \text {; }
\end{aligned}
$$

e) e, finalmente, $\{\beta\}_{\omega}=_{\text {df. }} \cup\left\{\{\beta\}_{n} \mid n \in \omega\right\},\{\beta\}_{\omega}$ é a inseminação de $\beta$ limitada porP.A..

1.28. Teorema. Se $\beta$ é uma sentença de $L_{\text {P.A. }}$ tal que T.P.A. $\cup\{\beta\} \# \overline{0}=\overline{1}$, então $\{\beta\}_{\omega} \neq \overline{0}=\overline{1}$.

Segue-se de 1.28 que:

1.29. Lema. Se $\alpha$ é uma sentença de $L_{P . A .}$ tal que $\alpha \notin$ T.P.A., então existe uma interpretação $\mathbb{N}\left(\{\beta\}_{\omega}\right)$ de $L_{\text {P.A.V. }}$ tal que, para qualquer $n, \mathbb{N}\left(\{\beta\}_{\omega}\right) \vDash \mathrm{V}(\lceil\cdots \mathrm{V}(\lceil\mathrm{V}(\lceil\alpha \mid)\rceil) \cdots\rceil)$. $n$ vezes

Portanto, temos que:

1.30. Teorema da descitação. Se $\alpha \in L_{\text {P.A. }}$ e $\operatorname{AcRM}(\mathrm{V}) \vdash \mathrm{V}([\cdots \mathrm{V}(\lceil\mathrm{V}(\lceil\alpha\rceil)]) \cdots\rceil)$, então P.A. $\vdash \alpha$ [cp. V-1.31, p. 114].

\section{B. Alguns resultados da teoria da derivação}

1.31. Teorema. Se $\operatorname{AcRM}(\mathrm{V}) \vdash \forall x(\varphi(x) \rightarrow \mathrm{V}(x))$, então existe $m$ tal que P.A. $\vdash \forall x\left(\varphi(x) \rightarrow \backslash \operatorname{SENT}(\mathrm{V})_{m} \backslash(x)\right)$. 
Prova. Seja $\alpha$ uma derivação de $\forall x(\varphi(x) \rightarrow \mathrm{V}(x))$ em $\operatorname{AcRM}(\mathrm{V}) \mathrm{e} \mathrm{V}_{i}^{\mathrm{R}}(x)$ a RV de maior complexidade de $\alpha$. Então, $/ \alpha /^{\mathrm{R}}$ será uma derivação de $\forall x\left(\varphi(x) \rightarrow \mathrm{V}_{i}^{\mathrm{R}}(x)\right)$ em P.A.. [cf. 1.10 e 1.11 p. 129]. Assim, uma vez que P.A. $\vdash \forall x\left(\mathrm{~V}_{i}^{\mathrm{R}}(x) \rightarrow \backslash \operatorname{SENT}(\mathrm{V})_{i} \backslash(x)\right)$, temos, também, que P.A. $\vdash \forall x\left(\varphi(x) \rightarrow \backslash \operatorname{SENT}(V)_{i} \backslash(x)\right)$.

Como no caso de AcM(V) [cf. V-1.33, p. 114], nosso sistema minimal AcRM(V) não pode estabelecer vários princípios gerais para os quais existem "versões metateóricas" da forma: "Se $\varphi$ é uma sentença de ..., então AcM(V) $\vdash \ldots \varphi \ldots$..

1.32. Teoremas. Se P.A. é consistente,

a) $\operatorname{AcRM}(\mathrm{V}) \Vdash \forall x(\operatorname{SENT}(\mathrm{V})(x) \rightarrow \mathrm{V}(\dot{\rightarrow}(x, x)))$;

b) $\operatorname{AcRM}(\mathrm{V}) * \forall x\left(\operatorname{Pr}_{\text {P.A. }}(x) \rightarrow \mathrm{V}(x)\right)$.

Seria em princípio possível estabelecer extensões de $\mathrm{AcRM}(\mathrm{V})$ análogas àquelas do sistema $\operatorname{AcM}(\mathrm{V})$; mais especificamente, poderíamos definir:

a) um sistema minimal $\operatorname{AcRM}(\mathrm{F})$ da acumulação enraizada-negativa análogo ao sistema $\operatorname{AcM}(\mathrm{F})$ [cf. p. 118];

b) um sistema minimal $\mathrm{AcRM}(\mathrm{VF})$ da dupla acumulação enraizada análogo ao sistema AcM(VF) [cf. p. 119].

c) Para, finalmente, estudarmos extensões análogas ao sistema $\operatorname{AcM}(\mathrm{VF})+E s q S$ [cf. p. 120].

O que não faremos nesse trabalho. Fica aqui, junto ao aprofundamento do estudo de AcRM(V), como sugestão de pesquisa. 


\section{§2. O sistema formal AcEM(V)}

Como nosso segundo e último exemplo de modificação das DVPs, introduziremos cláusulas de acumulação que, por assim dizer, aceitam teoremas da lógica (ou fórmulas válidas da linguagem em questão) como tendo uma complexidade aritmética finita qualquer, sendo possível, desse modo, derivar, trivialmente, certos princípios gerais da lógica relativos ao predicado de verdade. De fato, o sistema dessa seção é à primeira vista desesperadamente ad hoc; entretanto, ele se encontra aqui apenas para ilustrar uma possível direção de modificação de nossas definições parciais de verdade, posto que, acreditamos, alguma reflexão e trabalho acabem por conduzir a "sistemas estruturais" mais adequados.

\subsection{Definição de $\mathrm{V}_{0}^{\mathrm{E}}(x)$.}

a) Escreveremos $\mathrm{V}_{0}^{\mathrm{E}}(x)$ no lugar de $\mathrm{V}_{0}(x) \vee \operatorname{Pr}_{\varnothing}(x)$, onde é uma contrapartida de " $x$ é um teorema da lógica em $L_{\text {P.A.". }}$

Podemos, então, introduzir nossa forma geral (de fato, nada surpreendente) das definições parciais estruturais de verdade:

\subsection{Definição da Forma Geral das EVs.}

a) Escreveremos $\mathrm{V}_{n}^{\mathrm{E}}(x)$ no lugar de $\mathrm{V}_{n}(x) \vee \operatorname{Pr}_{\varnothing}(x)$.

Devemos notar que o uso de $\operatorname{Pr}_{\varnothing}(x)$ não passa de uma conveniência; poderíamos introduzir uma "definição de validade ou de verdade da lógica", $\mathrm{L}(x)$, intensionalmente correta (e de mesma extensão que $\operatorname{Pr}_{\varnothing}(x)$, é claro) por meio de métodos semânticos-o que estaria mais de acordo com os paradigmas de uma definição parcial de verdade.

Existe, ainda, outro caminho interessante nessa mesma linha de pensamento, na verdade, um caminho conceitualmente mais apropriado: poderíamos introduzir definições "aritmético-estruturais" de verdade. 
No caso, $\mathrm{V}_{0}^{\mathrm{E}}(x)$ seria o próprio $\mathrm{V}_{0}(x)$; mas quereríamos, por exemplo, que:

a) P.A. $\vdash \mathrm{V}_{1}^{\mathrm{E}}(\lceil\alpha \vee \overline{0}=\overline{0}\rceil)$, para qualquer sentença $\alpha$ de $L_{\text {P.A. }}$ (ou talvez de $L_{\text {P.A.V. }}$ ); mas que P.A. $\forall \mathrm{V}_{1}^{\mathrm{E}}([\sim \alpha \vee \alpha\rceil)$, por causa da necessidade "estrutural" dos dois conectivos de ' $\sim \alpha \vee \alpha$;

b) P.A. $\vdash \mathrm{V}_{2}^{\mathrm{E}}(\lceil\sim \alpha \vee \alpha\rceil)$ e P.A. $\vdash \mathrm{V}_{2}^{\mathrm{E}}(\lceil\sim \overline{0}=\overline{1} \rightarrow \alpha\rceil)$, para qualquer sentença $\alpha$ de $L_{P . A}$. (ou de L L.A.V.);

c) $\vdash \mathrm{V}_{3}^{\mathrm{R}}(\lceil\alpha \rightarrow(\overline{0}=\overline{0} \wedge \alpha)\rceil)$ e P.A. $\vdash \mathrm{V}_{3}^{\mathrm{R}}(\lceil(\beta \wedge \alpha) \rightarrow \alpha\rceil)$, para quaisquer sentenças $\alpha$ e $\alpha$ de $L_{\text {P.A. }}$ (ou $L_{\text {P.A.V. }}$; etc..

Infelizmente, uma definição rigorosa do conceito de "complexidade aritméticoestrutural de uma verdade da aritmética", que presumivelmente subjaz a esse tipo de definição parcial de verdade, traz consigo uma infinidade de dificuldades de ordem técnica-embora estejamos no domínio da semântica, essas questões conduzem a tópicos relativamente avançados da teoria da derivação (proof theory).

Devemos, portanto, abandonar aqui o estudo dessas definições aritméticoestruturais de verdade e dos sistemas formais correlatos, sugerindo fortemente um futuro estudo dessas questões.

Em todo caso (e é isso que nos interessa aqui), a própria possibilidade de estabelecermos definições parciais do tipo aritmético-estrutural parece tornar mais palatável o estudo da hierarquia, um tanto grosseira, baseada $\mathrm{emV}_{0}^{\mathrm{E}}(x)={ }_{\mathrm{df}} \mathrm{V}_{0}(x) \vee \operatorname{Pr}_{\varnothing}(x)$.

2.3. Definição. As fórmulas $\mathrm{V}_{0}^{\mathrm{E}}(x), \mathrm{V}_{1}^{\mathrm{E}}(x), \ldots, \mathrm{V}_{n}^{\mathrm{E}}(x), \ldots$ serão chamadas definições parciais estruturais de verdade ou, mais sucintamente, EVs.

2.4. Definição. As sentenças $\forall x\left(\mathrm{~V}_{n}^{\mathrm{E}}(x) \rightarrow \mathrm{V}(x)\right)$, onde $n$ é um número natural, serão chamadas cláusulas de acumulação estruturais ou, mais sucintamente, CAEs. 
2.5. Definição. $A c E M(V)$ é o sistema cujos axiomas são os axiomas de P.A. para $\mathrm{L}_{\text {P.A. }}$ e as sentenças $\forall x\left(\mathrm{~V}_{n}^{\mathrm{E}}(x) \rightarrow \mathrm{V}(x)\right)$, para todo $n$.

$\operatorname{AcEM}(\mathrm{V})$ é chamado sistema minimal (restrito) da acumulação estrutural.

Notemos que na teoria da acumulação baseada em $\mathrm{V}_{0}^{\mathrm{E}}(x)$ (e, presumivelmente, naquela baseada em definições aritmético-estruturais), diferentemente de $\operatorname{AcM}(V)$ e $\operatorname{AcRM}(\mathrm{V})$, poderíamos derivar princípios gerais, tais como:

2.6. Teoremas. $\operatorname{AcEM}(\mathrm{V}) \vdash \forall x(\operatorname{SENT}(x) \rightarrow \mathrm{V}(\dot{\rightarrow}(x, x)))$ [cp. p. 114 e p. 137].

Não obstante, temos que:

2.7. Teoremas. $A c E M(V)$ é conservativo sobre P.A..

Prazos a serem cumpridos barram, nesse momento, nosso avanço. Em todo caso, dada a fase atual da pesquisa, acreditamos que um sistema minimal híbrido, algo entre um sistema da acumulação estrutural-depois de devidamente refinado, estudado e compreendido (o caráter de $\mathrm{AcEM}(\mathrm{V})$ é meramente ilustrativo) - e o sistema $\mathrm{AcRM}(\mathrm{V})$ da seção anterior seria uma proposta, ao mesmo tempo, forte (reflitamos por um momento sobre nossos possíveis teoremas) e adequada (conservativa) do ponto de vista de um "predicado deflacionista" para as verdades da aritmética. 


\section{Considerações finais}

Posto tudo em pratos limpos, concluiremos basicamente que esse estudo permanece inacabado, mas que, não obstante, isso não é sinal de fraqueza e sim, de força. Nele algo poderia ser acrescentado, dele algo poderia ser subtraído; entretanto e em última análise, tais escolhas envolveriam sempre certa dose de arbitrariedade-no sentido em que poderíamos argumentar em favor de uma ou outra escolha particular e não no sentido em que não poderíamos defender nenhuma delas.

De fato, devido à imensa quantidade de caminhos e ramificações que as definições parciais de verdade e os sistemas formais que Ihes são correlatos nos apresentam, o desenvolvimento completo de tais potencialidades estaria, apartado por diversos tipos de limitações, bem distante de nossas capacidades e, sensatamente, do escopo desse trabalho; contudo, isso não quer dizer que nosso estudo não seja, desde já, promissor em alguns aspectos.

\section{Aspecto: os sistemas em si}

\section{A. Da correção intuitiva dos sistemas}

Os sistemas apresentados nesse estudo são todos corretos do ponto de vista lógico-intuitivo [cf. discussão, p. 34, inclusive, nota 38].

No caso do sistema $\operatorname{AcM}(\mathrm{V})$ e de outros sistemas do capítulo $\mathbf{V}$, a própria existência de "definições parciais"-ou seja, de fórmulas $\mathrm{V}_{0}(x), \mathrm{V}_{1}(x)$, etc. e $\mathrm{F}_{0}(x)$, $\mathrm{F}_{1}(x)$, etc. tais que, para qualquer número natural $n$ dado, se $\alpha$ é uma sentença de $L_{\text {P.A. }}$. cuja complexidade é $n$, então P.A. $\vdash \alpha \leftrightarrow \mathrm{V}_{n}(\lceil\alpha\rceil)$ e P.A. $\vdash \sim \alpha \leftrightarrow \mathrm{F}_{n}(\lceil\alpha\rceil)-$ conduz à ideia de acumulação e, portanto, de que sistemas como P.A. $+\left\{\forall x\left(\mathrm{~V}_{n}(x) \rightarrow\right.\right.$ $\mathrm{V}(x)) \mid n \in \omega\}$ e P.A. $+\left\{\forall x\left(\mathrm{~F}_{n}(x) \rightarrow \mathrm{F}(x)\right) \mid n \in \omega\right\}$ seriam sistemas minimais 
adequados da verdade e, respectivamente, da falsidade de sentenças da aritmética [cf., também, discussão em I-\$2.4, pp. 36-39].

No caso de $\operatorname{AcRM}(\mathrm{V})$, uma vez que existem sentenças $\alpha$ de complexidade $n$ em $\mathrm{L}_{\text {P.A.V. }}$ tais que AcRM(V) $\Vdash \alpha \leftrightarrow \mathrm{V}_{n}^{\mathrm{R}}(\lceil\alpha\rceil)$ e que as RVs, por assim dizer, cuidam de ou enxergam as sentenças de L.A.V. [cf. VI-1.14, p. 131], as definições parciais enraizadas de verdade não são "definições parciais" no mesmo sentido que as DPVs. Não obstante, nossas RVs [cf. VI-1.5, p. 127] são contruídas, apesar da adição da cláusula para 'V', de forma totalmente análoga às DPVs, de modo que a própria construção é bastante simples e natural, não sendo, de fato, nada além da internalização do procedimento recursivo da definição de verdade tarskiana, acrescido de um princípio interno de acumulação que estabelece que se $\alpha$ é uma sentença verdadeira de complexidade $n$, então $\mathrm{V}(\lceil\alpha\rceil)$ deverá ser uma sentença verdadeira de complexidade $n+1$ [cf. VI-1.5.g), p. 127].

De fato, desde que nem todos os bicondicionais de Tarski são deriváveis em AcRM(V) [cf. VI-1.25, p. 134], uma defesa ampla e articulada da correção lógico-intuitiva de $\operatorname{AcRM}(\mathrm{V})$ poderia ser convertida em um ataque ao próprio dogma tarskiano do princípio de citação-descitação, ou seja, à "convention T" enquanto condição de adequação material do predicado de verdade ${ }^{(1)}$-ataque, esse, feito do ponto de vista clássico e não das lógicas alternativas (deviant logics).

Notemos, ainda, que, uma vez que o paradoxo do mentiroso é, em certo sentido, intuitivamente correto $^{(2)}$ e está relacionado ao caráter auto-referencial do predicado de

1. Uma afirmação de Shapiro feita em um contexto estranho à nossa discussão imediata é, por isso, sintomática: "We learned from Tarski (1935) that any decent theory of truth will have to have, as a consequence, each instance of truth schema [...]" (grifo nosso) [“Deflation and Conservation”, p. 104].

2. Nesse primeiro sentido, a antinomia de Russell é, por exemplo, correta do ponto de vista da teoria das extensões e, daí, da teoria ingênua dos conjuntos, mas não presumivelmente do ponto vista da hierarquia cumulativa de Zermelo, ou seja, da noção cumulativa de conjunto [no caso da hierarquia de Zermelo cf., por exemplo, Maddy, Naturalism in Mathematics, 1997, pp. 19-20]. 
verdade da linguagem coditiana, nosso estabelecimento da consistência relativa de $\operatorname{AcRM}(\mathrm{V})$ [cf. VI-1.12, p. 130] ou, pelo menos, algumas indicações no sentido desse estabelecimento poderiam ser tomadas, devido ao caráter auto-referencial do sistema, como condição necessária à correção lógico-intuitiva de $\operatorname{AcRM}(\mathrm{V})^{(3)}$.

As mesmas considerações relativas ao sistema $\mathrm{AcM}(\mathrm{V})$ - naturalidade, correção, etc. - se aplicam ao sistema AcEM(V), no pior dos casos, por meio de um detour pelo conceito de verdade aritmético-estrutural [cf. discussão, pp. 137-138].

\section{B. Do deflacionismo}

Nossos sistemas minimais se adequam satisfatoriamente a várias das mais diversas propostas deflacionistas.

Naquilo que é muitas vezes tomado como pedra de toque do deflacionismo ${ }^{(4)}$, $\operatorname{AcM}(V), \operatorname{AcM}(V F), \operatorname{AcRM}(V)$ e $\operatorname{AcEM}(V)$ são todos conservativos sobre a teoria de base (e, em alguns casos, sobre a teoria pura da identidade). Além disso, como querem muitos deflacionistas, AcRM(V) permite um tratamento auto-referencial do predicado de verdade e AcEM(V) estabelece formas generalizadas de certos princípios lógicos fundamentais (apesar de serem ambos conservativos sobre P.A.).

\section{Das extensões}

Nossos sistemas são adequados como base para sistemas mais fortes, como ficou exemplificado com o sistema $\mathrm{AcM}(\mathrm{VF})+\operatorname{Esq} S$ [cf. p. 120] e sugerido na possível hibridização entre AcRM(V) e AcEM(V) [cf. p. 139].

3. Nesse último sentido, todo peso recai no 'lógico' de 'lógico-intuitiva', nosso predicado de verdade deve ser, de alguma forma, logicamente tratável.

4. O argumento de que devemos tomar a conservatividade de nossa teoria da verdade (em relação à teoria de base) como condição de adequação dessa primeira ao deflacionismo é, no mais das vezes, fundamentado por algum tipo de identificação entre não-substancial (não-inflacional) e conservativo [para uma discussão e outras referências, cf. Shapiro, 2004, sobretudo, pp.108-112]. 


\section{Da metateoria}

Muitos dos aspectos metateóricos (tanto da teoria da derivação quanto da teoria dos modelos) dos nossos sistemas são interessantes: a compatibilidade entre nossos sistemas minimais e o trivialismo por exemplo; contudo, é justamente no estudo mais detido dos modelos não-dialéticos de $\operatorname{AcRM}(\mathrm{V})$ que parece residir alguns dos aspectos mais promissores da metateoria dos nossos sistemas de acumulação, e. g., os conceitos de enraizamento, modelo padrão de $\operatorname{AcRM}(V)$ e inseminação limitada por P.A. [cf. pp. 132 e pp. 134-135].

\section{2o Aspecto: a comparação entre teorias}

A comparação entre nossos sistemas e outros apresentados na literatura parece iluminar reciprocamente as diversas propostas. Nesse sentido deixamos algumas respostas e questões mais precisas:

Leia-se $\mathrm{A} \subseteq \mathrm{B}$ como A é uma subteoria de B,

a) $\mathrm{CT}(\mathrm{V}) \subseteq \mathrm{AcM}(\mathrm{V}), \operatorname{AcM}(\mathrm{V}) \nsubseteq \mathrm{BT}(\mathrm{V})$ e $\mathrm{BT}(\mathrm{V}) \subseteq \mathrm{AcM}(\mathrm{VF})+E s q S$ [cf. V-1.11, p. 105 e V-2.14, p. 121];

b) $\mathrm{CUT}(\mathrm{V}) \subseteq \mathrm{AcM}(\mathrm{V})$ ? $\mathrm{BUT}(\mathrm{V}) \subseteq \mathrm{AcM}(\mathrm{VF})+\operatorname{Esq} S$ ?

E, além disso, um repertório imenso de questões mais vagas:

a) Qual é a relação entre $\operatorname{AcRM}(\mathrm{V})$ e os outros sistemas auto-referenciais ou typefree apresentados na literatura (e. g., FS ou Friedman-Sheard Theory e KF ou KripkeFeferman Theory [cf. Halbach, 2011, pp. 159-162 e pp. 195-202, respectivamente])?

b) Qual é a relação entre $\mathrm{AcEM}(\mathrm{V})$ e os sistemas composicionais apresentados na literatura (e.g., C(V) e CV [cf. pp. 33-34])? 


\section{3 - Aspecto: A pesquisa futura}

Como deve estar claro, há muito a ser feito do ponto de vista das teorias da acumulação da verdade.

Do ponto de vista filosófico, nosso estudo de "ocasião" sobre procedimentos de extensão de formalismos [cf. p. 11] encontrou discussões vívidas relativas à natureza do predicado de verdade e, incidentalmente, a imensa bibliografia que sustenta e move essas discussões. De modo que o balizamento de nossas "teorias da acumulação" em relação aos argumentos apresentados nessa bibliografia e a indicação dessas primeiras como alternativas viáveis às teorias deflacionistas da literatura se converte em um projeto filosófico atraente e, mesmo, irrecusável do ponto de vista da discussão contemporânea sobre o conceito de verdade.

Do ponto de vista lógico-sistemático, para que novos resultados realimentem toda discussão anterior, tudo é ainda mais óbvio: novos teoremas e propriedades metateóricas devem ser estabelecidos, novas extensões de nossos sistemas minimais devem ser propostas, a análise comparativa deve ser aprofundada, etc. 


\section{Obras citadas}

Ackermann, W., 1937. 'Die Widerspruchsfreiheit der Allgemeinen Mengenlehre', Mathematische Annalen, Volume: 114, pp. 305-315.

Aristóteles, 1969. Metafísica. Porto Alegre, Editora Globo.

Boolos, G. and Jeffrey, R., 1980 (2nd ed.). Computability and Logic. Cambrigde University Press.

Burgess, J. P., 2004. 'Is There a Problem about the Deflationary Theory of Truth', em Principles of Truth, editado por V. Halbach e L. Horsten. Frankfurt, Ontos Verlag, pp. 37-55.

Buss, S. R., 1998. 'First-Order Proof Theory of Arithmetic', in Handbook of Proof Theory, edited by S. R. Buss. Amsterdam, Elsevier, pp 79-147.

Curry, H. B., 1977. Foundations of Mathematical Logic. Dover Publications.

Davidson, D., 2006. 'Truth and Meaning' (1967), em The Essential Davidson, Oxford University Press, pp. 155-170.

2005. 'The Folly of Trying to Define Truth' (1996), em Truth, Language and History, Clarendon Press, pp. 19-36.

Epstein, R. L., 2006. Classical Mathematical Logic. Princeton University Press.

Feferman, S., 1960. 'Arithmetization of metamathematics in a general setting', Fundamenta Mathematicae, No. 49, pp.35-92.

1962. 'Transfinite recursive progressions of axiomatic theories', Journal of Symbolic Logic, Vol. 27, No. 3, pp. 259-316. 
Feferman, S., 1991. 'Reflecting on incompleteness', Journal of Symbolic Logic, Vol. 56, No. 1, pp. 1-49. , 1996. 'Gödel's Program for new axiomas: Why, where, how and what?', em Gödel'96, editado por Peter Hájek, AK Peters, pp. 3-22.

Field, H., 2008. Saving Truth from Paradox. Oxford University Press.

Franzén, T., 2004. Inexhaustibility. Wellesley. A K Peters, Ltd..

Gödel, K., 2004. 'On formally undecidable propositions os the Principia Mathematica and related systems l' (1931), em The Undecidable, edited by M. Davis, New York, Dover Publications, pp.4-38.

Hájek, P. \& Pudlák, P., 1998. Metamathematics of First-Order Arithmetic, Springer.

Halbach, V., 2011. Axiomatic Theories of Truth, Cambrigde University Press.

Horwich, P., 2004. 'A Defense of Minimalism', em Principles of Truth, editado por V. Halbach e L. Horsten. Frankfurt, Ontos Verlag, pp. 57-73.

Kaye, R., 1991. Models of Peano Arithmetic, Clarendon Press.

Kaye, R. \& Wong, T. L., 2007. 'On interpretations of arithmetic and set theory', Notre Dame Journal of Formal Logic, Vol. 48, No. 4 (2007), pp. 497-510.

Kirkham, R.L., 2001. Theories of Truth: a Critical Introduction, MIT Press.

Kripke, S., 1975. 'Outline of a theory of truth', Journal of Philosophy, 72, pp. 690-712.

Künne, W., 2003. The Concepts of Truth. Clarendon Press. 
Maddy, P., 1997. Naturalism in Mathematics. Oxford University Press. 2011. Defending the Axioms. Oxford University Press.

Mendelson, E., 1997 (4th ed.) Introduction to Mathematical Logic. Chapman \& Hall.

Priest, G., 2006a (2nd ed.). In Contradiction. Oxford University Press. 2006b. Doubt Truth to Be a Liar. Oxford University Press.

Quine, W.V., 1970. Philosophy of Logic. Prentice-Hall Inc..

Shapiro, , 2004. 'Deflation and Conservation', em Principles of Truth, editado por V. Halbach e L. Horsten. Frankfurt, Ontos Verlag, pp. 103-128.

Shoenfield, J. R., 1967. Mathematical Logic. Reading. Addison-Wesley Publishing Company.

Smith, P., 2007. An Introduction to Gödel's theorems. Cambrigde. Cambrigde University Press.

Smullyan, R. M., 1992. Gödel Incompleteness Theorems, Oxford University Press. 1995. First-Order Logic. New York, Dover Publications , Inc..

Soames, S., 1999. Understanding Truth, Oxford University Press.

Tarski, A., Mostowski, A. and Robinson, R. M., 1971. Undecidable Theories. Amsterdam. North-Holland Publishing Company.

Tarski, A., 1935. 'Der Wahrheitsbegriff in den formalisierten Sprachen', em Studia philosophica, vol. I, 1935, pp. 261-405. 
Tarski, A., 1956. 'The Concept of Truth in Formalized Languages' (tradução de J. H. Woodger), em Logic, Semantics, Metamathematics, pp. 152-278, Claredon Press. , 1944. 'The Semantic Conception of Truth', Philosophy and Phenomenological Research, Vol. 4, No. 3 (Mar. 1944), pp.341-376. 1994 (4th ed.). Introduction to Logic and the Methodology of Deductive Sciences. Oxford University Press.

Turing, A. M., 2004. 'Systems of logic based on ordinals' (1939), em The Undecidable, edited by M. Davis, New York, Dover Publications. pp.155-222. 